\title{
Studies of the
}

\section{San Francisco Bay, California, Estuarine Ecosystem}

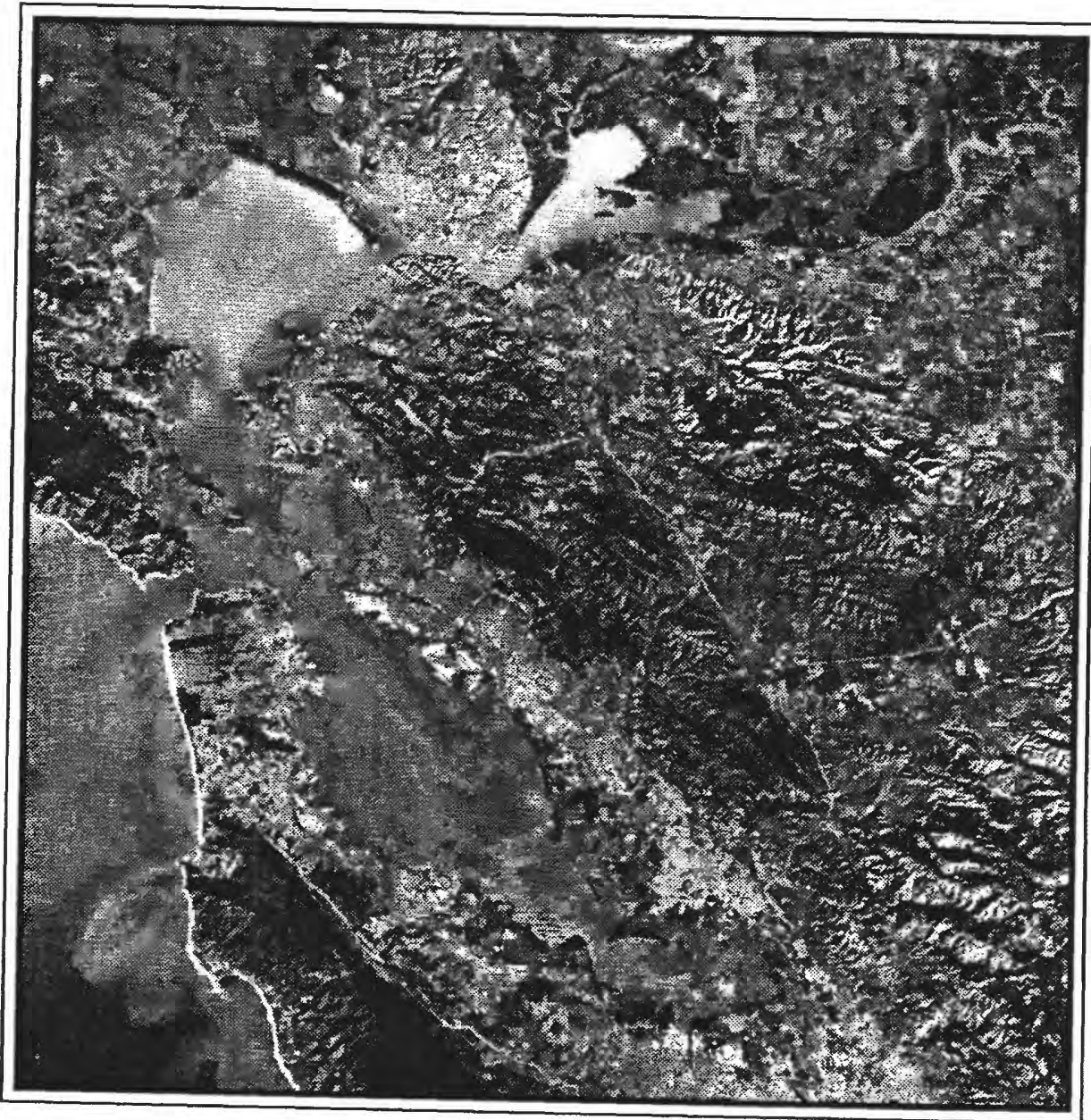

\section{Regional monitoring \\ program results, 1997}

BY

Jelriza I. Baylosis, Brian E. Cole, and James E. Cloern

Open-File Report 98-168

Menlo Park, California

US Department of the Interior

US Geological Survey

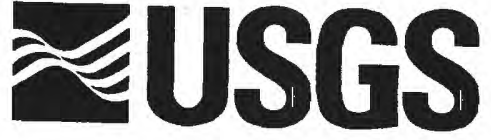


STUDIES OF THE SAN FRANCISCO BAY, CALIFORNIA, ESTUARINE ECOSYSTEM.

REGIONAL MONITORING PROGRAM RESULTS, 1997

By Jelriza I. Baylosis, Brian E. Cole and James E. Cloern

U.S. Geological Survey

Open-File Report 98-168

Menlo Park, California

1998 


\section{U.S. DEPARTMENT OF THE INTERIOR \\ BRUCE BABBITT, Secretary \\ U.S. GEOLOGICAL SURVEY \\ Thomas J. Casadevall, Acting Director}

Any use of trade, product, or firm names is for descriptive purposes only and does not imply endorsement by the U.S. Government.

For additional information write to:

Copies of this report can be purchased from:

James E. Cloern

Water Resources Division

U.S. Geological Survey

345 Middlefield Road, MS-496

Menlo Park, California 94025
U.S. Geological Survey

Earth Science InformationCenter

Open-File Reports Section

Box 25286, MS 517

Denver Federal Center

Deriver, Colorado 80225 


\section{CONTENTS}

\section{Page}

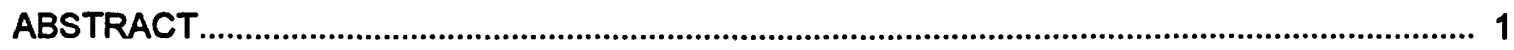

INTRODUCTION

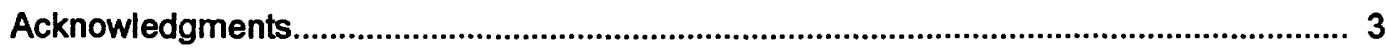

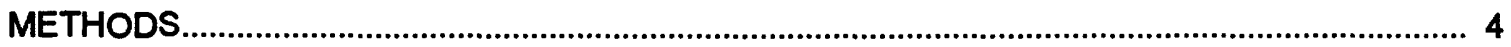

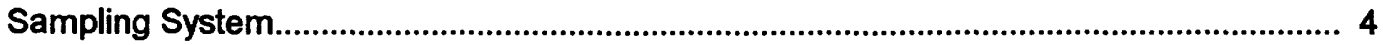

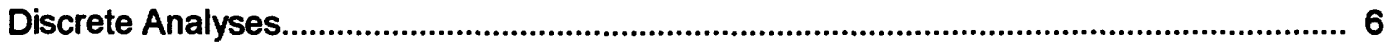

Instrument Calibration................................................................................................ 7

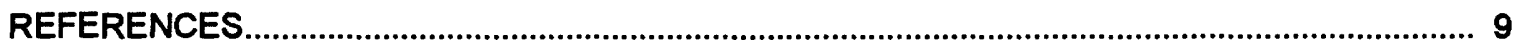

Figure 1 - Map of San Francisco Bay estuary showing locations of water sampling stations......... 11

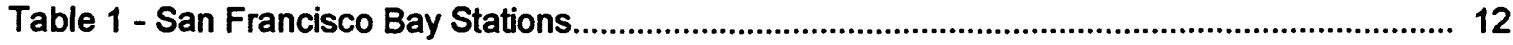

Table 2 - Dates of Cruises and Stations Occupied................................................................... 15

APPENDIX A Data Summaries of Hydrographic Properties....................................................... A-1 


\section{CONVERSION FACTORS}

Multiply

meter (m)

nanometer $(\mathrm{nm})$

gram

kilogram

milligram

liter
By

3.28

$2.94 \times 10^{-8}$

$2.2 \times 10^{-3}$

2.2

$2.2 \times 10^{-6}$

1.06
To Obtain

foot

inch

pound

pound

pound

quart

Temperature is given in degrees Celsius $\left({ }^{\circ} \mathrm{C}\right)$ and can be converted to degrees Fahrenheit ( ${ }^{\circ} \mathrm{F}$ ) using the following equation:

$$
{ }^{\circ} \mathrm{F}=1.8\left({ }^{\circ} \mathrm{C}\right)+32
$$



MONITORING PROGRAM RESULTS, 1997

By Jelriza I. Baylosis, Brian E. Cole and James E. Cloern

\section{ABSTRACT}

As part of a regional monitoring program, water samples were collected in the San Francisco Bay estuary during 20 cruises from January through November 1997. Conductivity, temperature, light attenuation, turbidity, oxygen, and in-vivo chlorophyll fluorescence were measured longitudinally and vertically in the main channel of the estuary from south of the Dumbarton Bridge in the southern part of the Bay to Rio Vista on the Sacramento River. Discrete water samples were analyzed for chlorophyll a, phaeopigments, suspended particulate matter, and dissolved oxygen. Water density was calculated from salinity, temperature, and pressure (depth), and is included in the data summaries. 


\section{INTRODUCTION}

A major goal of the San Francisco Estuary Project's Comprehensive Conservation and Management Plan (CCMP) is "to improve the scientific basis for managing natural resources within the estuary through an effective monitoring and research program" (San Francisco Estuary Project Management Committee, 1994). The CCMP more specifically recommends the development and implementation of a regional monitoring strategy to integrate and expand upon existing efforts and to eventually be part of a comprehensive San Francisco Bay Regional Monitoring Program (RMP). The first version of a regional monitoring strategy was eventually developed and implemented as the Regional Monitoring Program for Trace Substances. This program is a multi-component sampling program designed to provide information on status and trends of water quality and trace substances in the San Francisco Bay-Delta Estuary. One of the components is a sampling program conducted by the U.S. Geological Survey (USGS) to characterize the seasonal distributions of water quality along the entire Bay-Delta estuary. This report provides results of that program for 1997.

A regional monitoring program must cover many types of resources, including pelagic and benthic channel habitats, shoal habitat, wetlands, river channels, sloughs, and small bays and harbors. The USGS component of the RMP focuses on the pelagic channel habitat. The objective of this component is to provide a high-resolution description of basic constituents of water quality, which can be used along with other information to determine the suitability of habitat for aquatic resources and provide a context for understanding the dynamic changes in trace substances and their effects. Measurements of physical (salinity, temperature, suspended particulate matter, and light penetration), chemical (dissolved oxygen), and biological characteristics (chlorophyll a) are included in this report. The purpose of this document is to provide a comprehensive listing of the 1997 results. An interpretive analysis, based on these data, will be published in the 1997 RMP Annual Report. All data in this report can be viewed and accessed over the Internet, at the following URL: http://sfbay.wr.usgs.gov/access/wqdata 
Samples were collected throughout San Francisco Bay monthly from January through November 1997. During January, February, March, and April when a phytoplankton bloom was expected in South Bay (stations 21 through 36), the frequency of cruises in South Bay was increased to 2-4 cruises each month. Sampling sites are shown in figure 1, their locations and water depth are identified in table 1, and the sampling dates are listed in table 2. A Sea-Bird Electronics conductivity-temperature-depth (CTD) data acquisition system, Sea Tech in-situ fluorometer, Sea-Bird Electronics oxygen sensor, D \& A Instruments optical backscatter sensor (OBS), and LI-COR quantum sensor were used to measure vertical distributions of conductivity (salinity), temperature, chlorophyll fluorescence, oxygen concentration, turbidity, and solar irradiance, respectively, at each station. Discrete chlorophyll a, oxygen, and suspended particulate samples were also collected at selected sites to calibrate the fluorometer, oxygen sensor, and OBS sensor on each sampling date.

\section{Acknowledgments}

This measurement program was supported primarily by the U.S. Geological Survey Toxic Substances Hydrology Program. Additional support was provided by participants in the Regional Monitoring Program for Trace Substances, including municipal and industrial dischargers, cooling water and storm water dischargers, and dredgers. We thank these participants for their financial support and their foresight in appreciating the need to view the Estuary as a whole and to manage it on a regional basis. We also acknowledge the Regional Water Quality Control Board for its oversight and coordination of the Regional Monitoring Program, and the San Francisco Estuary Institute for its essential role in management of that program. 


\section{METHODS}

\section{Sampling System}

In-situ measurements were made from the USGS Research Vessel Polaris with a high speed data acquisition system. At each station, a Sea-Bird Electronics (SBE) underwater unit (SBE-9) was lowered through the water column. Attached to the data acquisition unit were the following sensors: SBE-4 conductivity sensor, SBE-3 temperature probe, Paroscientific digiquartz pressure transducer, Sea Tech in-vivo fluorometer, SBE-13 oxygen sensor, D\&A Instruments OBS3 optical backscatter sensor, and LI-COR Instruments 1925 quantum sensor. With this instrument package, vertical distributions of conductivity, temperature, depth (pressure), chlorophyll fluorescence, oxygen concentration, turbidity, and solar radiance (photon flux density) were measured throughout the water column.

The instrument package was lowered through the water at about 0.5 meter per second to within 1 meter of the bottom. Signals from the conductivity, temperature, pressure, fluorescence, oxygen, OBS, and light sensors were digitized in the underwater unit (SBE-9) at 24 scans per second, resulting in a vertical sampling interval of about $2 \mathrm{~cm}$. The data were transmitted to a deck unit through a single-conductor armored cable, displayed and stored on a shipboard computer. To conserve space in this report, the data presented in Appendix $A$ are centered averages of about 50 data points collected over each 1-meter depth interval. For example, the one-meter values listed in Appendix $A$ are averages of all measurements made between depths of 0.5 and 1.5 meters.

The conductivity sensor was a 2-terminal, 3 electrode (platinum), flow-through sensor. This sensor was accurate within 0.0004 Siemens per meter and had a resolution of $5 \times 10^{-5}$ Siemens per meter (Sea-Bird Electronics). Temperature (TEMP) was measured with a Wien Bridge type resistance thermistor. This sensor was accurate to $\pm 0.002^{\circ} \mathrm{C}$ and had a resolution of $0.0005^{\circ} \mathrm{C}$ (Sea-Bird Electronics). The conductivity and temperature probes were recalibrated in December 1996 at the Sea-Bird Electronics Calibration Center, Bellevue, Washington. Values for salinity 
(SALIN) were calculated from conductivity and temperature using the algorithm supplied with the Sea-Bird Electronics software, based on the equations of Millero and others (1981) and Millero and Poisson (1981). Water density, as sigma-theta (SIGT), derived from salinity, temperature, and pressure, was also calculated with the Sea-Bird software package. Calculations of sigma-t were based on the equations of Fofonoff and Millard (1983). Sampling depths (DEPTH), derived from changes in pressure measured by the Paroscientific digiquartz transducer, were accurate within 0.01 meter.

Chlorophyll a fluorescence was measured with a Sea Tech submersible pulsed-light fluorometer, which had a flash rate of 5 times per second. Because the sampling rate of the fluorometer was about one fifth that of the Sea-Bird underwater unit ( 5 times per second as compared with 24 times per second), the fluorescence data reported here are 3-meter running averages of the measured values in order to smooth the variability associated with this mismatch of sampling frequencies. Hence, the vertical resolution of chlorophyll a fluorescence reported here was about 3 meters.

Dissolved oxygen concentrations (OXYG) were measured with an SBE-13 oxygen electrode that contains a Beckman polarographic element. The electrode had a mean accuracy of $0.14 \mathrm{mg} \mathrm{O}_{2}$ per liter, compared with the discrete measurements described below. Dissolved oxygen concentration was calculated with the Sea-Bird software package, based on the equation of Owens and Millard (1985).

Optical backscatter, or turbidity, was measured using a D \& A Instruments OBS-3 sensor which had an $875 \mathrm{~nm}$ infrared source and silicon photodetectors. During the months of August, September and October the OBS sensor was calibrated to accomodate a wider range of turbidity. This accounts for the difference in sensitivity of the OBS sensor during these months compared to the rest of the year.

Visible light was measured with a LI-COR 1925 quantum sensor sensitive to photosynthetically active radiation $(400-700 \mathrm{~nm})$. The light extinction coefficient (EXCOF) was 
calculated as the slope of the least-squares regression of the natural logarithm of irradiance $\left(\ln \left(\mathrm{I}_{\mathrm{z}}\right)\right)$ against the depth $(\mathrm{Z})$ where the irradiance was measured.

\section{Discrete Analyses}

Discrete water samples for chlorophyll a, phaeopigments, dissolved oxygen, and suspended particulate matter (SPM) were collected at selected stations coincident with the lowering of the submersible instrument package. Water samples were collected from 1 meter above the bottom with a Niskin water sampling bottle and from 2 meters below the surface through a centrifugal pump with an intake at the ship's bow. Each discrete sample for chlorophyll a (DISCR $\mathrm{CHL}$ a) and phaeopigments (PHA) was filtered at less than $12 \mathrm{~cm} \mathrm{Hg}$ vacuum onto a Gelman AVE glass fiber filter and immediately frozen. The cold air-dried filter was ground in 90 percent acetone. After extraction for $12-24$ hours at -5 to $-10^{\circ} \mathrm{C}$, samples were centrifuged and absorbances of the extracts were determined on a Hewlett Packard 8452A diode array spectrophotometer. The acetone extracts were acidified to measure phaeopigments (Riemann, 1978). Chlorophyll a and phaeopigment concentrations were calculated using Lorenzen's (1967) equations.

Dissolved oxygen concentrations (DISCR OXYG) were measured in water collected from the bow pump into $300 \mathrm{~mL}$ biological oxygen demand (BOD) bottles. The bottles were filled from the bottom and allowed to overflow for approximately 30 seconds. Winkler reagents (Strickland and Parsons, 1972) were added immediately and bottles were stored with water in their cap wells and covered by a snap-fit plastic cap. Before beginning the titrations, the samples were acidified and $100.2 \mathrm{~mL}$ of sample was collected by autopipette. The sample was titrated with $0.01 \mathrm{~N}$ sodium thiosulfate dispensed by a Metrohm autotitrator using the potentiometric titration method of Granéli and Granéli (1991). Potassium iodate standardization of the sodium thiosulfate was conducted under identical procedures to eliminate problems associated with iodine volatilization (Knapp and others, 1991). Standard normalities were determined separately for each new preparation of sodium thiosulfate. 
Suspended particulate matter (DISCR SPM) was measured gravimetrically, as described by Hager (1994). Between $0.10-1.0 \mathrm{~L}$ of water were filtered onto preweighed $0.4-\mu \mathrm{m}$ pore size polycarbonate membrane filters and allowed to air dry for 48-72 hrs. Filters were then reweighed and a correction for salt on the filters was made to calculate the concentration of suspended particulate matter.

\section{Instrument Calibration}

Estimates of chlorophyll a at each depth were derived from linear regressions of measured chlorophyll a (DISCR CHL a) against fluorescence (FLUOR). The slope (B) and intercept (A) from the regressions were used to calculate chlorophyll a concentrations (CALC CHL a) from the fluorescence values (CALC CHL $a=A+B \times$ FLUOR). If calculated chlorophyll a was less than or equal to zero or if the output voltage (fluorescence) was less than 0.2 , the result was not printed. Regression coefficients are presented at the end of each daily data summary. The coefficient of determination $\left(r^{2}\right)$ indicates the strength of the linear relation between discrete chlorophyll a and fluorescence. Caution needs to be exercised in using calculated chlorophyll a values when this coefficient is less than $\mathbf{0 . 7 0}$. Also note that some of the regressions resulted in nonzero intercepts, which indicate that there was fluorescence in the water not associated with particulate chlorophyll a. Calculated chlorophyll a concentration divided by discrete chlorophyll a plus phaeopigment concentration ([CALC CHL a]/DISCR CHL a+PHA]) gives the proportion of active chlorophyll a to total pigments (CHL a/ a + PHA), including chlorophyll degradation products.

The oxygen electrode measurements (OXYG) were calibrated using discrete dissolved oxygen measurements (DISCR OXYG). These estimates of dissolved oxygen concentrations with depth (CALC OXYG) were derived from a linear regression of discrete dissolved oxygen measurements (DISCR OXYG) and measurements with the electrode (OXYG). Calculation of percent oxygen saturation (\% OXY SAT) for each calculated oxygen value was based on equation 4 from Weiss (1970). 
Calculated SPM concentrations (CALC SPM) with depth were derived from a linear regression of the discrete measurements of SPM concentration (DISCR SPM) and the optical backscatter (OBS) voltage. Significant differences among the calibration regression coefficients for individual cruise dates necessitated unique instrument calibrations for the fluorometer, optical backscatter, and oxygen sensor for each day of sampling. 


\section{REFERENCES}

Fofonoff, N.P. and Millard, R.C., Jr., eds., 1983, Algorithms for computation of fundamental properties of seawater: UNESCO Technical Papers in Marine Science, Number 44, UNESCO, Paris.

Grańeli, W. and Grańeli, E., 1991, Automatic potentiometric determination of dissolved oxygen: Marine Biology v. 108, p. 341-348.

Hager, S.W., 1994, Dissolved nutrient and suspended particulate matter data for the San Francisco Bay Estuary, California. October 1991 through November 1993: U.S. Geological Survey. Open-File Report 94-471, 53 p.

Knapp, G.P., Stalcup, M.C., and Stanley, R.J, 1991, lodine losses during Winkler titrations: Deep-Sea Research v. 38, p. 121-128.

Lorenzen, C.J., 1967, Determination of chlorophyll and phaeopigments. Spectrophotometric equations: Limnology and Oceanography, v. 12, p. 343-346.

Millero, F.J., Chen, C.T., Bradshaw, A., and Schleicher, K., 1981, Summary of data treatment for the international high pressure equation of state for seawater: UNESCO Technical Papers in Marine Science, Number 38, UNESCO, Paris.

Millero, F.J. and Poisson, A., 1981, Summary of data treatment for the international one atmosphere equations of state for seawater: UNESCO Technical Papers in Marine Science, Number 38 , UNESCO, Paris.

Owens, W.B. and Millard, R.C., Jr, 1985, A new algorithm for CTD oxygen calibration: Journal of Physical Oceanography. v. 15, p. 621-631.

Riemann, B., 1978, Carotenoid interference in spectrophotometric determination of chlorophyll degradation products from natural populations of phytoplankton: Limnology and Oceanography, v. 23, p. 1059-1066.

San Francisco Estuary Project Management Committee, 1994, Comprehensive conservation 
and management plan: U.S. Environmental Protection Agency. 236 p.

Strickland, J.D.H. and Parsons, T.R, 1972, A practical handbook of seawater analysis: Fisheries Research Board Canada, Ottawa, Canada. $310 \mathrm{p}$.

Weiss, R.F., 1970, The solubility of nitrogen, oxygen and argon in water and seawater: DeepSea Research v.17, p. 721-735. 


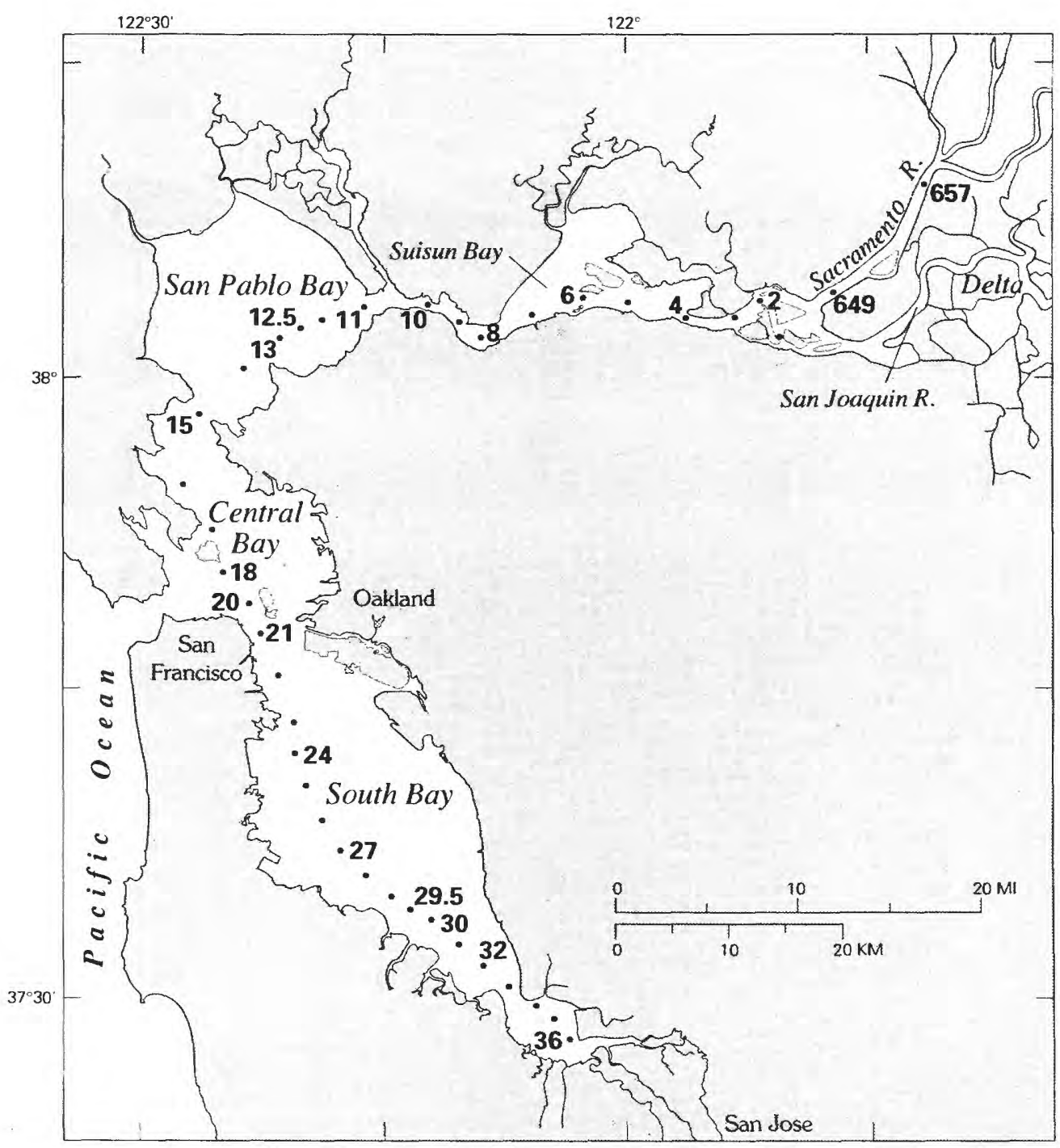

Fig 1 - Map of San Francisco Bay estuary showing locations of water sampling stations (See table 1 for identification of station) 
Table 1 - San Francisco Bay Stations (locations shown on figure 1)

\begin{tabular}{|c|c|c|c|c|}
\hline $\begin{array}{l}\text { Station } \\
\text { Number }\end{array}$ & $\begin{array}{l}\text { General } \\
\text { Location }\end{array}$ & $\begin{array}{l}\text { North } \\
\text { Latitude }\end{array}$ & $\begin{array}{l}\text { West } \\
\text { Longitude }\end{array}$ & $\begin{array}{l}\text { Depth, in meters, } \\
\text { at mean low water }\end{array}$ \\
\hline 657 & Rio Vista & $38^{\circ} 8.9^{\prime}$ & $121^{\circ} 41.3^{\prime}$ & 10.1 \\
\hline 649 & Sacramento River & $3.7^{\circ}$ & $48.0^{\circ}$ & 10.1 \\
\hline . 2 & Chain Island & $3.8^{\prime}$ & $51.3^{\circ}$ & 11.3 \\
\hline 3 & Pittsburg & $3.0^{\circ}$ & $52.7^{\circ}$ & 11.3 \\
\hline 4 & Simmons Point & $2.9^{\circ}$ & $56.1^{\circ}$ & 11.6 \\
\hline 5 & Middle Ground & $3.6^{\circ}$ & $58.8^{\circ}$ & 9.8 \\
\hline 6 & Roe Island & $3.9^{\circ}$ & $122^{\circ} 2.1^{\circ}$ & 10.1 \\
\hline 7 & Avon Pier & $2.9^{\circ}$ & $5.8^{\prime}$ & 11.6 \\
\hline 8 & Martinez & $1.8^{\prime}$ & $9.1^{\circ}$ & 14.3 \\
\hline 9 & Benicia & $3.0^{\circ}$ & $10.4^{\prime}$ & 34.4 \\
\hline 10 & Crockett & $3.6^{\circ}$ & $12.5^{\prime}$ & 17.7 \\
\hline 11 & Mare Island & $3.7^{\circ}$ & $15.8^{\circ}$ & 15.5 \\
\hline 12 & Pinole Shoal & $3.4^{\circ}$ & $18.7^{\prime}$ & 8.8 \\
\hline 12.5 & Pinole Point & $2.4^{\circ}$ & $18.9^{\circ}$ & 6.7 \\
\hline 13 & N. of Pinole Point & 1.7 & $22.2^{\prime}$ & 9.8 \\
\hline
\end{tabular}




\begin{tabular}{|c|c|c|c|c|}
\hline $\begin{array}{l}\text { Station } \\
\text { Number }\end{array}$ & $\begin{array}{l}\text { General } \\
\text { Location }\end{array}$ & $\begin{array}{l}\text { North } \\
\text { Latitude }\end{array}$ & $\begin{array}{l}\text { West } \\
\text { Longitude }\end{array}$ & $\begin{array}{l}\text { Depth, in meters, } \\
\text { at mean low water }\end{array}$ \\
\hline 14 & "Echo" Buoy & $0.4^{\prime}$ & $24.3^{\prime}$ & 13.1 \\
\hline 15 & Point San Pablo & $37^{\circ} 58.5^{\prime}$ & $122^{\circ} 26.2^{\prime}$ & 22.9 \\
\hline 16 & "Charlie" Buoy & $54.9^{\prime}$ & $26.8^{\prime}$ & 12.8 \\
\hline 17 & Central Bay Deep & $50.8^{\prime}$ & $24.7^{\prime}$ & 25.0 \\
\hline 18 & Point Blunt & $50.8^{\prime}$ & $25.3^{\prime}$ & 43.0 \\
\hline 20 & Blossom Rock & $49.2^{\prime}$ & $23.6^{\prime}$ & 18.2 \\
\hline 21 & Bay Bridge & $47.3^{\prime}$ & $21.5^{\prime}$ & 17.4 \\
\hline 22 & Potrero Point & $45.9^{\prime}$ & $21.5^{\prime}$ & 18.0 \\
\hline 23 & Hunter's Point & 43.7 & $20.2^{\prime}$ & 20.1 \\
\hline 24 & Candlestick Point & $41.9^{\prime}$ & $20.3^{\prime}$ & 11.0 \\
\hline 25 & Oyster Point & $40.2^{\prime}$ & $19.5^{\prime}$ & 8.8 \\
\hline 26 & San Bruno Shoal & $38.1^{\prime}$ & $18.8^{\prime}$ & 9.8 \\
\hline 27 & San Francisco Airport & $37.1^{\prime}$ & $17.5^{\prime}$ & 13.0 \\
\hline 28 & N. of San Mateo Bridge & $36.1^{\prime}$ & $16.2^{\prime}$ & 16.2 \\
\hline 29 & S. of San Mateo Bridge & $34.8^{\circ}$ & 14.7 & 14.6 \\
\hline 29.5 & Steinberger Slough & $34.1^{\prime}$ & $13.1^{\prime}$ & 14.6 \\
\hline
\end{tabular}




\begin{tabular}{|c|c|c|c|c|}
\hline $\begin{array}{l}\text { Station } \\
\text { Number }\end{array}$ & $\begin{array}{l}\text { General } \\
\text { Location }\end{array}$ & $\begin{array}{l}\text { North } \\
\text { Latitude }\end{array}$ & $\begin{array}{c}\text { West } \\
\text { Longitude }\end{array}$ & $\begin{array}{l}\text { Depth, in meters, } \\
\text { at mean low water }\end{array}$ \\
\hline 30 & Redwood Creek Entrance & $33.3^{\prime}$ & 11.4 & 12.8 \\
\hline 31 & Coyote Hills & $31.7^{\prime}$ & $9.5^{\prime}$ & 13.7 \\
\hline 32 & Ravenswood Point & $31.1^{n}$ & $8.0^{n}$ & 12.8 \\
\hline 33 & Dumbarton Bridge & $37^{\circ} 30.5^{\prime}$ & $122^{\circ} 7.3^{\prime}$ & 11.6 \\
\hline 34 & Newark Slough & $29.7^{\prime}$ & $5.6^{\prime}$ & 7.9 \\
\hline 35 & Mowry Slough & $28.8^{\prime}$ & $4.8^{\prime}$ & 8.5 \\
\hline 36 & Calaveras Point & $28.3^{\prime}$ & $3.9^{\prime}$ & 7.9 \\
\hline
\end{tabular}


Table 2 - Dates of Cruises and Stations Occupied in 1997

South Bay
CTD cast
No data

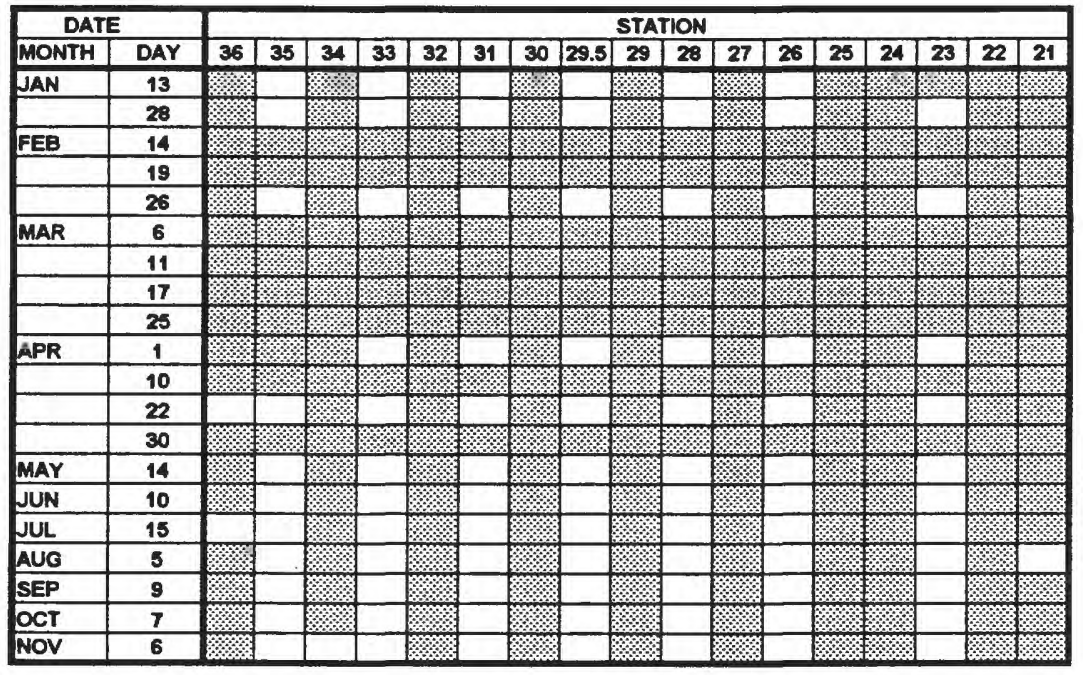

North Bay

\begin{tabular}{|c|c|c|c|c|c|c|c|c|c|c|c|c|c|c|c|c|c|c|c|c|c|c|}
\hline \multicolumn{2}{|c|}{ DATE } & \multicolumn{21}{|c|}{ STATION } \\
\hline MONTH & DAY & 657 & 649 & 2 & 3 & 4 & 5 & 6 & 7 & 8 & 9 & 10 & 11 & 12 & 13 & 14 & 15 & 16 & 17 & 18 & 20 & Other Stations \\
\hline JAN & 13 & & & & & & & & & & & & & 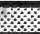 & & & & & & & . & 12.5 \\
\hline JAN & 28 & & & & & & & & & & & & & & & & & & & & 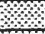 & 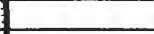 \\
\hline FEB & 26 & & & & & & & & & & & & & $\%$ & & & & & & & x & \\
\hline APR & 1 & & & & & & & & & & & & & & & & & & & & $\alpha$ & \\
\hline APR & 22 & & & & & & & & & & & & & 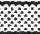 & & & & & & & 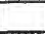 & \\
\hline MAY & 14 & & & & & & & & & & & & & & & s & & & 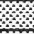 & & 椾 & \\
\hline JUN & 10 & & & & & & & & 8 & & & & \% & \% & \% & 型 & $\%$ & 勧 & 厓 & & & \\
\hline JUL & 15 & & 䇈 & & & & & & & & $\alpha$ & & & 3 & 繗 & . & & & & & 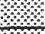 & \\
\hline AUG & 5 & & & & & & & & & & \% & 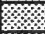 & & & & & 8 & & & & 勧 & \\
\hline SEP & 9 & & 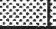 & & & & & & 8 & & & & 沙 & & 簽 & \% & 8 & \% & & & 留 & \\
\hline Oा & 7 & & & & & & & & & & & & & 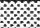 & & \% & & & & & 奴 & \\
\hline NOV & .6 & & \% & & & & & & & & 荻 & & F & & & \% & & & \% & & & \\
\hline
\end{tabular}





\section{APPENDIX A}

Data Summaries of Hydrographic Properties

Explanation of Abbreviations and Units

\begin{tabular}{|c|c|c|}
\hline Variable & Abbreviation & Units \\
\hline station & STN & \\
\hline time at which sample was taken & TIME & local time \\
\hline depth at which sample was taken & DEPTH & meters \\
\hline measured chlorophyll a & DISCR CHL a & mg per cubic meter \\
\hline $\begin{array}{l}\text { fraction of measured pigments and } \\
\text { degradation products attributable to } \\
\text { chlorophyll a }\end{array}$ & $\mathrm{CHL}$ a/a+PHA & fraction \\
\hline fluorescence & FLUOR & volts \\
\hline calculated chlorophyll a & CALC CHL a & mg per cubic meter \\
\hline measured dissolved oxygen & DISCR OXYG & $\mathrm{mg} \mathrm{O}_{2}$ per liter \\
\hline dissolved oxygen from CTD & OXYG & $\mathrm{mg} \mathrm{O} \mathrm{O}_{2}$ per liter \\
\hline calculated dissolved oxygen & CALC OXYG & $\mathrm{mg} \mathrm{O}$ per liter \\
\hline calculated percent oxygen saturation & $\%$ OXY SAT & percent \\
\hline measured suspended particulate matter & DISCR SPM & mg per liter \\
\hline optical backscatter & OBS & volts \\
\hline calculated suspended particulate matter & CALC SPM & mg per liter \\
\hline extinction coefficient & EXCOF & per meter \\
\hline salinity & SALIN & practical salinity units \\
\hline temperature & TEMP & degrees Celsius \\
\hline sigma-theta & SIGT & kg per cubic meter \\
\hline
\end{tabular}


(588888888888888888888888888

这

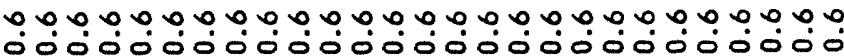

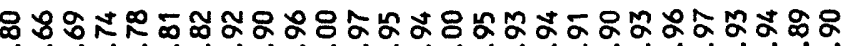

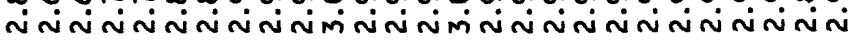

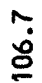

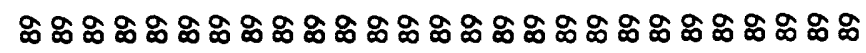

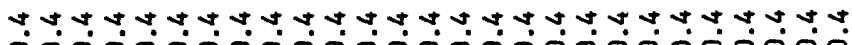

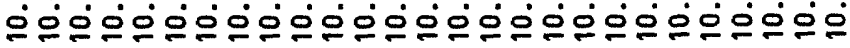

n--

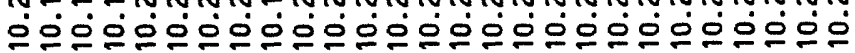

$\stackrel{n}{0}$

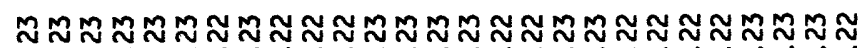
000000000000000000000000000

î.

$\hat{0}$

웅롱

픔

000000000000000000000000000

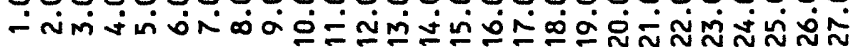

崖

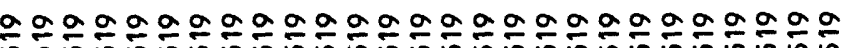

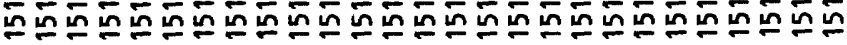

000000000000000000000000000

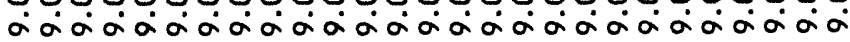

888888888888888 ó00000000000

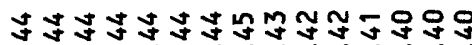

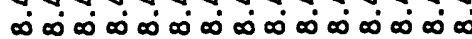

ิㅡㅇํㅇㅇㅇㅇㅇㅇㅇㅇㅇㅇㅇㅇㅇㅇㅇㅇㅇㅇㅇㅇㅇㅇํㅇ

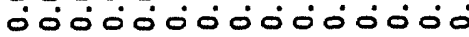

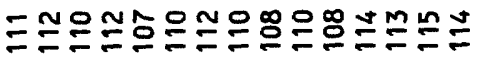

スロ

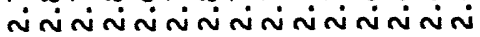

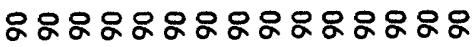

m m 000000 m

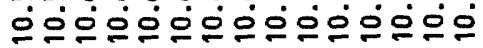

mununununutyotaty 옹ㅇㅇㅇㅇㅇㅇㅇㅇㅇㅇㅇㅇㅇㅇㅇ

000000000000000

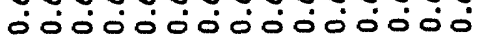

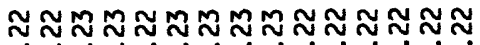
0000000000000

$\stackrel{\circ}{\circ}$

in:

000000000000000

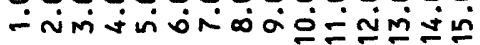

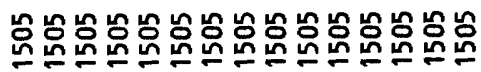

000000000000000

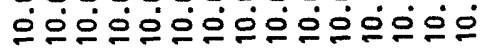




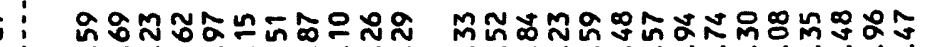

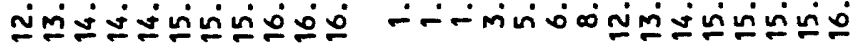

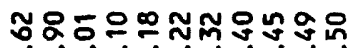

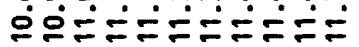

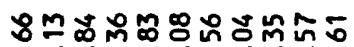

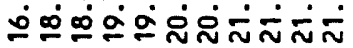

๓ $8 \div \approx 8 \div ะ 5888$ Niminimivisaso

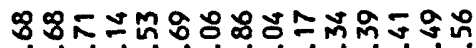

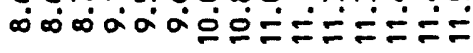

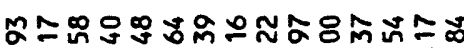

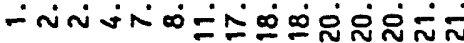
$\stackrel{\sim}{\sim}$

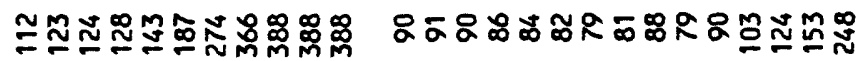

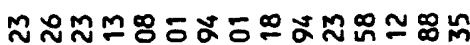
Niñiñinisinimio

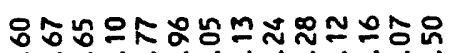

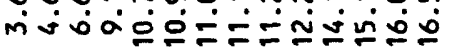

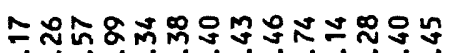

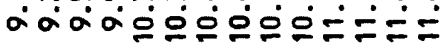

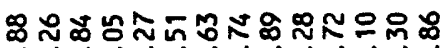

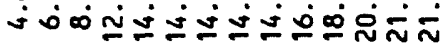

$\dot{s}$

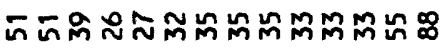

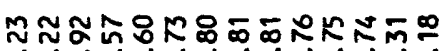

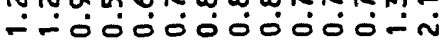

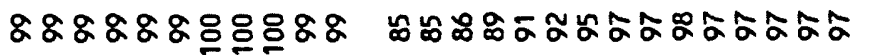

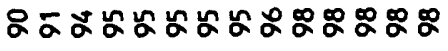

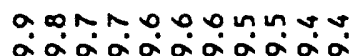

T⿻o.o.0.0\%nummmn

O- - ONRNON

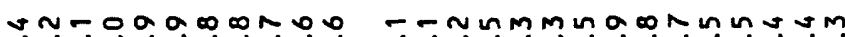

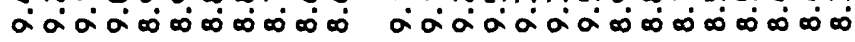

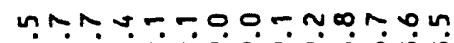

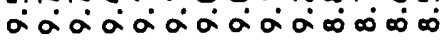

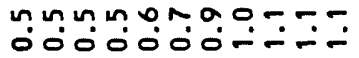

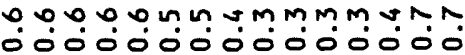

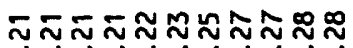
ல்0ல்0ல்0ல்

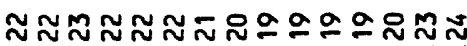
0ं000000000000

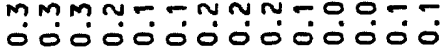

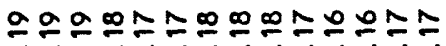

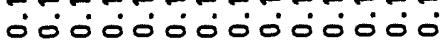

$\overline{0}$

$\dddot{\sim}$

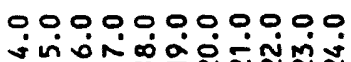

000000000000000 -

0.000000000000

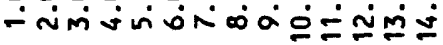

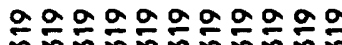

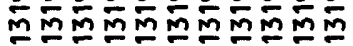

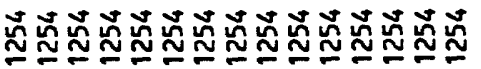

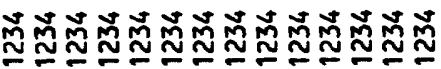

0.0000000000

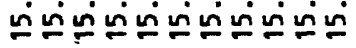

000000000000000

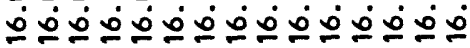

00000000000000

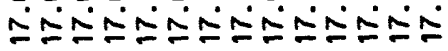


8888888888888888888888 $\therefore 0^{\circ}$

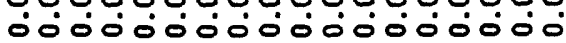

영웡ㅇㅇ $\infty \infty \infty$

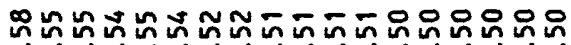
i

ㅊํㅇํํㅇํำ कं०்

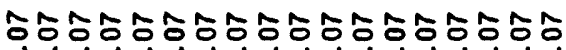
000000000000000

ֻั.

$\cong ゚ \cong \cong$

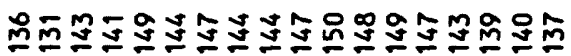

용ํำ inimi

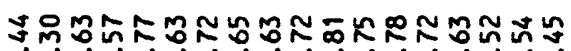

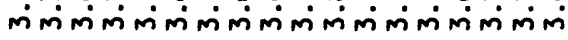

888888 ํํำำ

000000000

$888 \div 5 \%$ ํํำำㅇำ

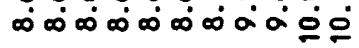

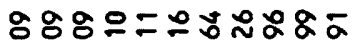

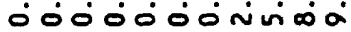

$\tilde{0}$

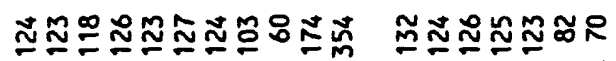

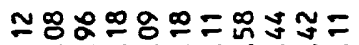
miñiminimiñ
$888588 \%$

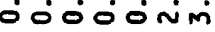

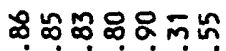
$\infty \infty \dot{\infty} \infty \dot{\infty} \infty \dot{\alpha}$

ニニลับ유요

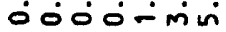

$\tilde{n}$

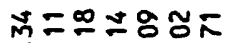
miniminin:

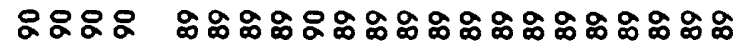

num nn ㅇํㅇํำ ㅁํㅇㅇํㅇํㅇ 요요

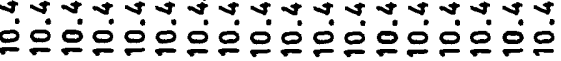
N MMNMNMNNMMNMmMMNM

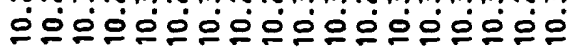

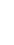

பீல்

กำก ००0.0

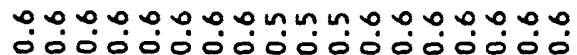

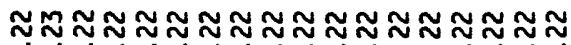
000000000000000000

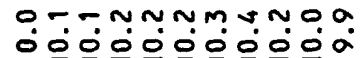

onnoagonanm åa
タะタะัณณ

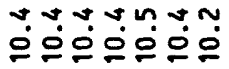

N N M ㅇํㅇㅇㅇ웅

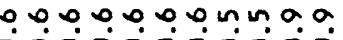
00000000000

$\approx \approx \approx \approx \approx \approx \approx \pi \overline{2} \approx \mathbb{2}$ 0000000000

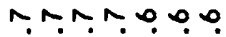
ல000000

$\mathbb{N} \mathfrak{N} \mathbb{N} \sim \mathbb{N}$ 0000000

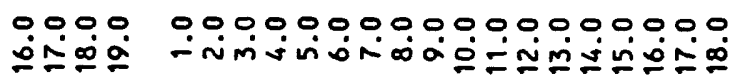

总总总总

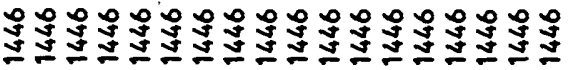

웅유웅

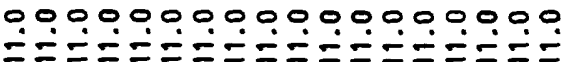

00000000000

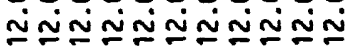

non $n$ in un nn

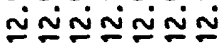


与 \&

m 0 -

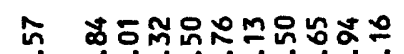

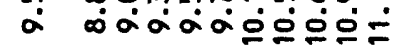

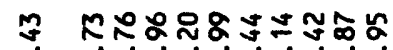

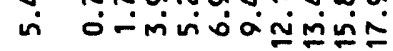

$\stackrel{\circ}{\sim}$

$\frac{m}{\delta}$

㝴蛋

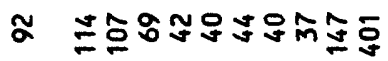

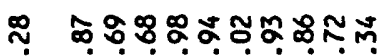

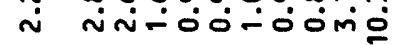

$\stackrel{m}{\stackrel{m}{0}}$

ชัญ

$\hat{\sigma}$

$\stackrel{m}{m}$

)

善 눟

芑롱

운

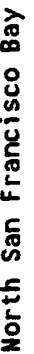

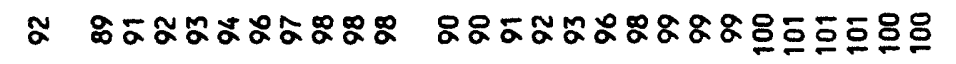

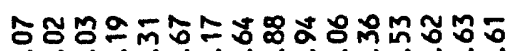

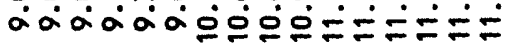

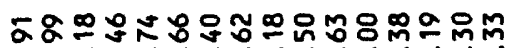

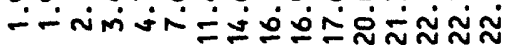

$\stackrel{?}{\dot{0}}$

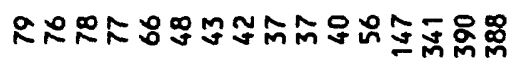

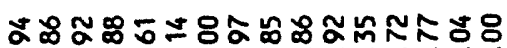

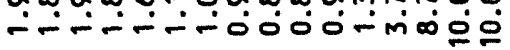

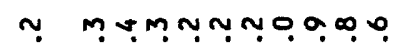

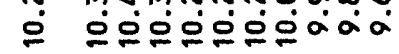

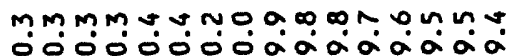

$\infty$ onogamunno

a 옹aaaa

$\stackrel{+}{\circ}$

- Donatammano

0 0000000000

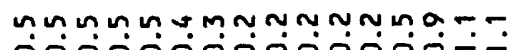

OO-ONnOOMmmoaño

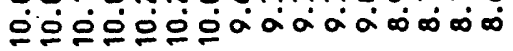

ก พก๊กำำำก 0000000000

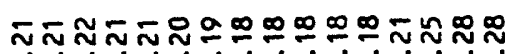

$\stackrel{\circ}{\circ}$

$\overline{\operatorname{s}}$

$\ddot{\circ}$

a.

0.0000000000

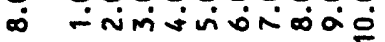

0000000000000000

-

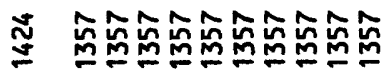

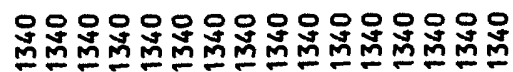

0000000000000000

$\because 0000000000$

ஸ $\dot{m} \dot{m} \dot{m} \dot{m} \dot{m} \dot{m} \dot{m}$

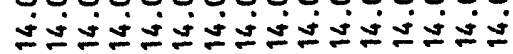

$\dot{8}$

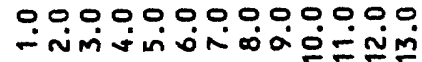

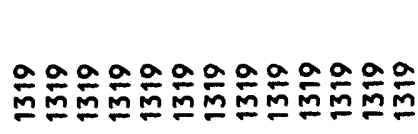

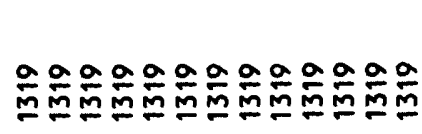

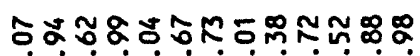

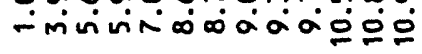

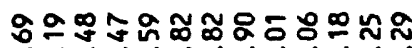

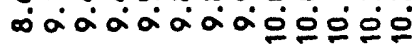

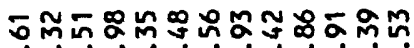

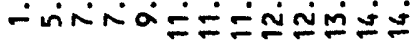

in

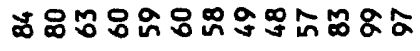

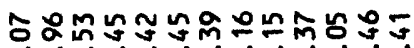

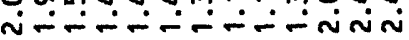

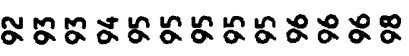

nm-nNoOOaAana

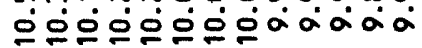

$4-\infty \infty \infty n n n n y+4 m$

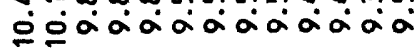

$\stackrel{m}{\circ}$

nnntutummmun 000000000000

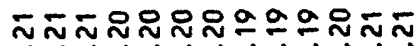
0ं0000000000

$$
\begin{aligned}
& \frac{n}{0} \\
& m \\
& 0
\end{aligned}
$$

0000000000000

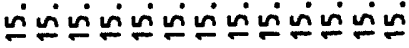


ำ

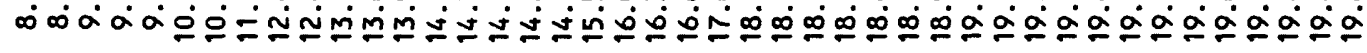

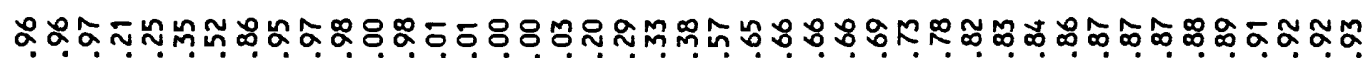

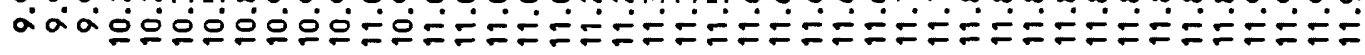

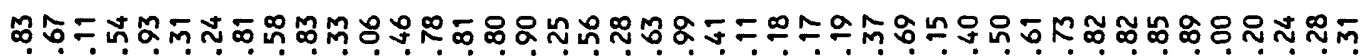

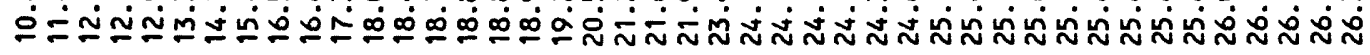
$\because$

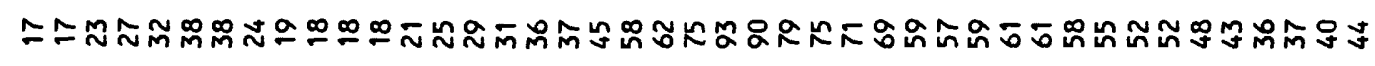
mై

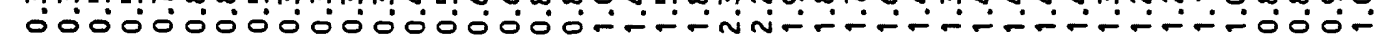
$\stackrel{\square}{\sim}$

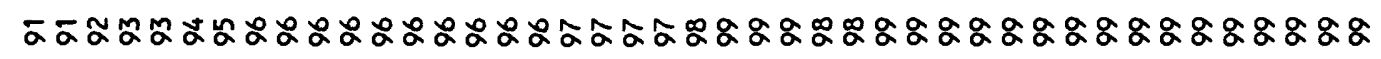

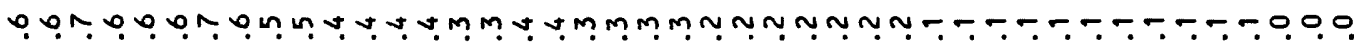

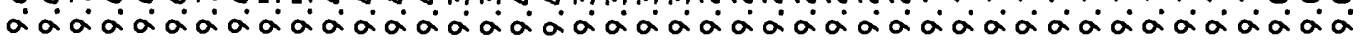
a. a o a a o -

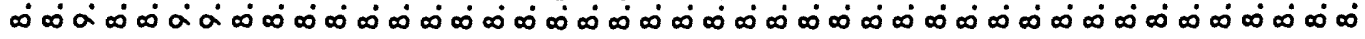

$$
\stackrel{0}{a}
$$

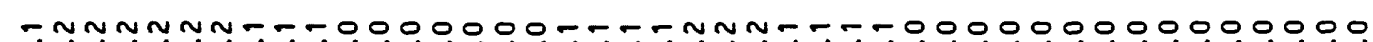

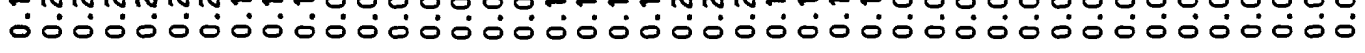

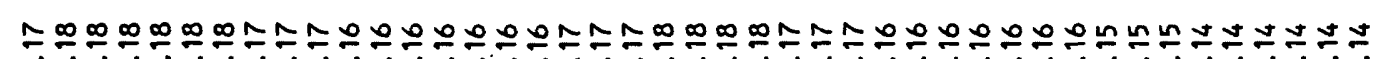

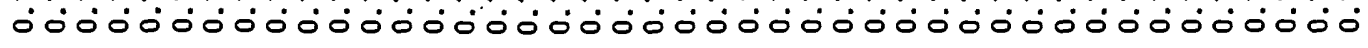

$$
\begin{aligned}
& m \\
& 0 \\
& 0 \\
& 0
\end{aligned}
$$

00000000000000000000000000000000000000000000

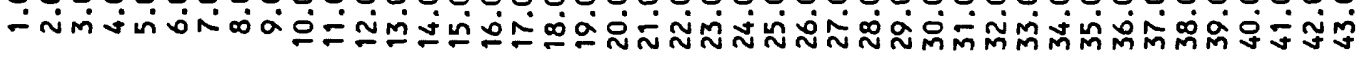

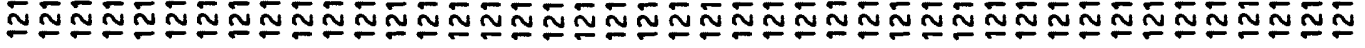

0000000000000000000000000000000000000000000 


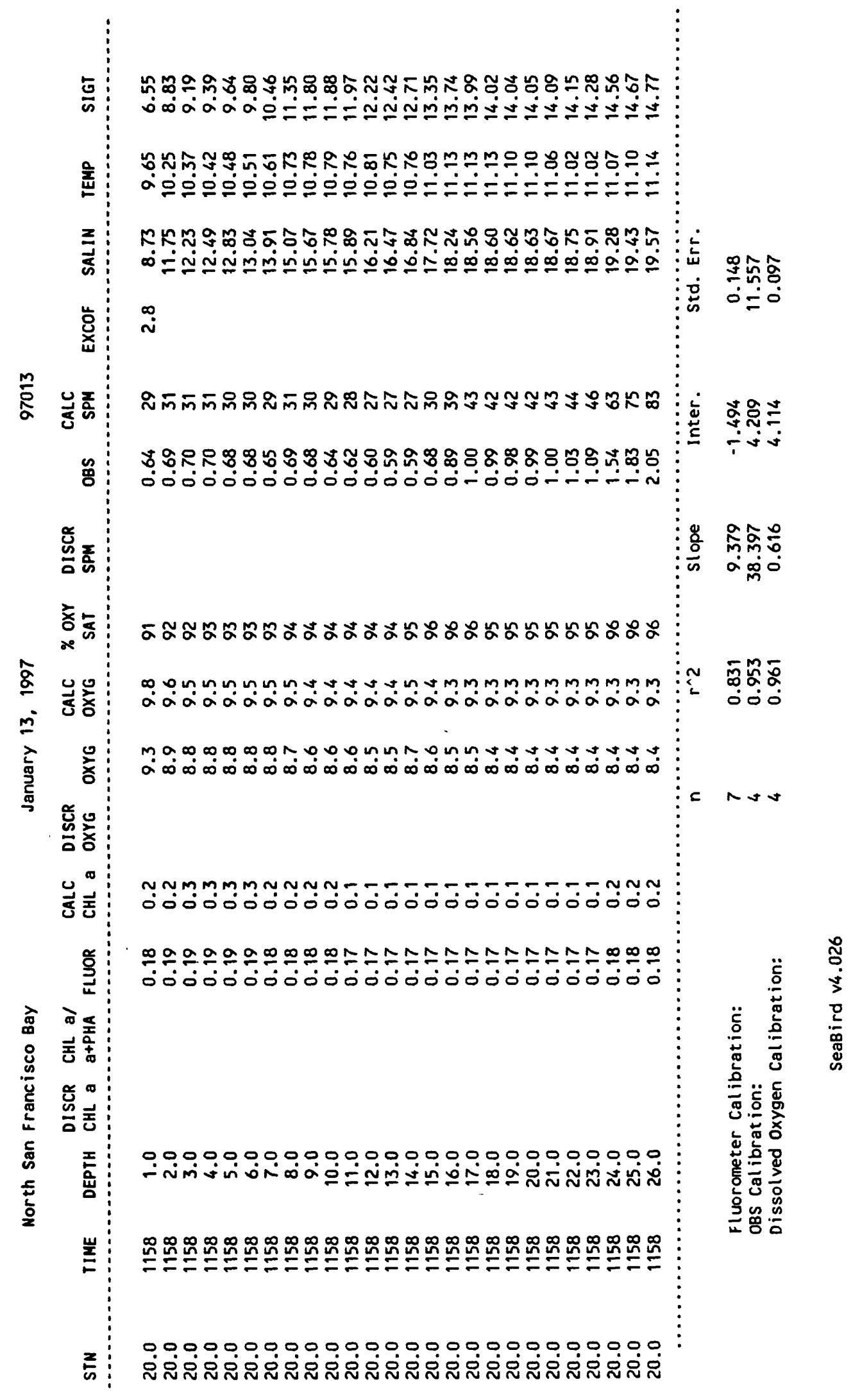




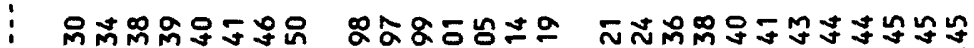

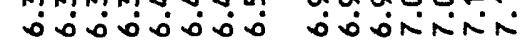

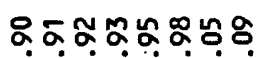

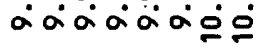

ษำㄴํํํํㅛㅇㅛ $\infty \infty_{\infty}^{\infty} \infty \infty_{\infty}^{\infty} \infty \infty_{\infty}^{\infty}$

$\stackrel{9}{=}$

商

㝴

噐

苑

炭

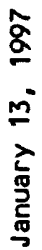

这

蒿옹

㝴돈

象

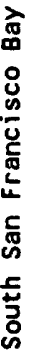

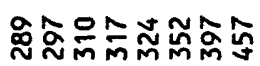

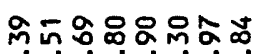

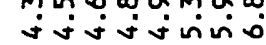

à

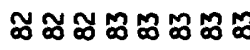

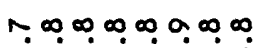

$\infty \infty \infty \infty \infty \infty \infty \infty$

$\infty \infty \infty \infty \infty a \infty \infty$

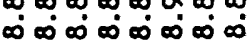

$\stackrel{\infty}{\infty}$

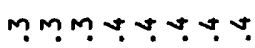

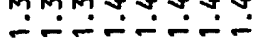

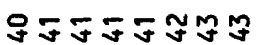

O.0.0000.00

$=$

今ó

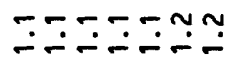

mmmmm绝品

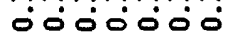

๙

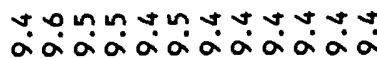

0.00009 .9

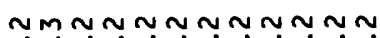

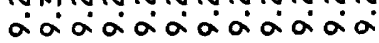

má

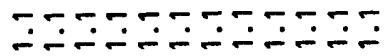

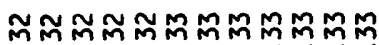

00000000000

I

$\stackrel{?}{0}$

$\stackrel{9}{-}$

00000000

- imivivisio

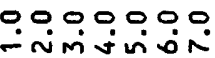

$\dot{a}$

$\stackrel{a}{\circ}$

000000000000

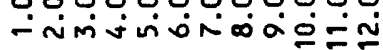

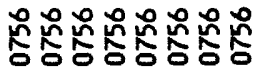

$\varliminf_{0}^{\infty} \frac{\infty}{8} \frac{\infty}{8} \frac{\infty}{8} \frac{\infty}{8} \frac{\infty}{8} \frac{\infty}{8}$

0000000000000 - imvivion

웅우웅우웅

0000000

000000000000

종

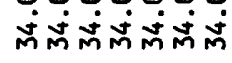

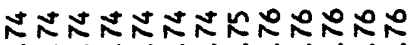

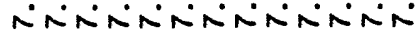

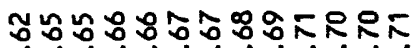

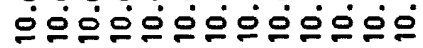

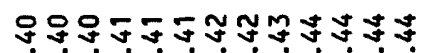
옹ㅇㅇㅇㅇㅇㅇㅇㅇㅇㅇㅣ

$\stackrel{n}{m}$

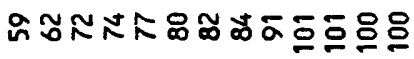

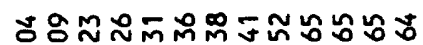

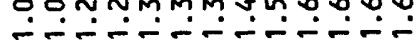

m.

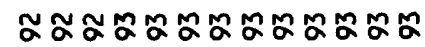

ب0

mmmmmmUvUmmm

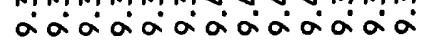

$\tilde{a}$

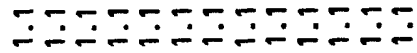

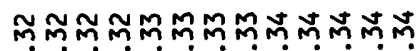
000000000000

$\stackrel{m}{m} \quad \bar{n}$

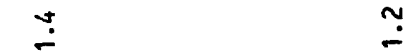

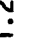

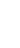




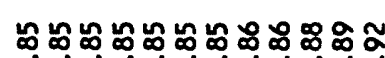

芯皆

爮麦

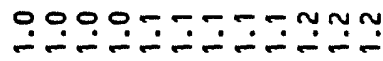

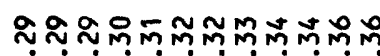
00000000000

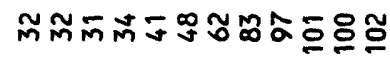

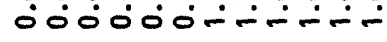

ทำ

000000000000 NNANRNANRNA

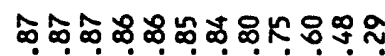

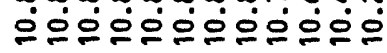

员 엉ㅇㅇㅇㅇㅇㅇㅇㅇㅇㅇ

m.

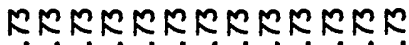

NANANANANANA

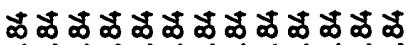

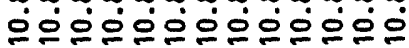

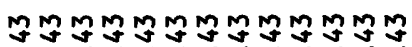
웅ㅇㅇㅇㅇㅇㅇㅇㅇㅇㅇㅇ

$\dot{m}$

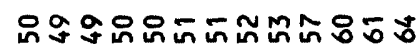

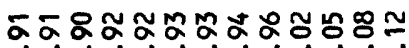
000000000---

$\stackrel{\infty}{\infty}$

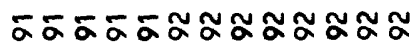

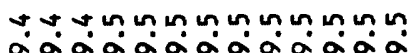

ก บ บ Nmmmmmmmm

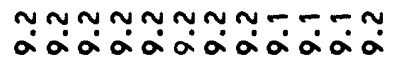
$a$

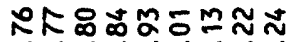
NiNiñ⿻

KKNTNKKKR 응ㅇㅇㅇㅇㅇㅇㅇ

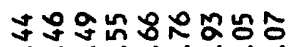

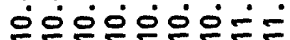

$\bar{i}$

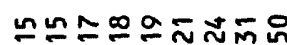

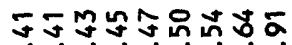
00000000

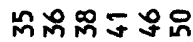
000000

$$
\stackrel{0}{\infty}
$$

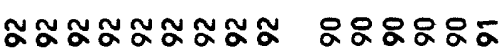

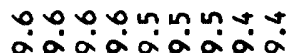

Mmmmmmm N åąająå

$$
\stackrel{0}{a}
$$

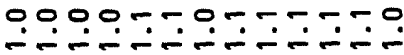

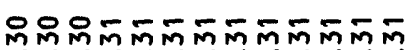

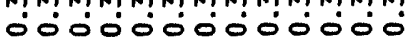

$\begin{array}{ll}\stackrel{0}{0} & \tilde{0} \\ 0 & \stackrel{0}{\sim}\end{array}$

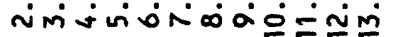

0000000000000

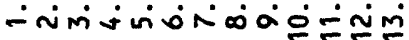

กั.

$\dddot{2}$

$a \alpha \infty \infty \infty \infty \infty \infty$ 000000000

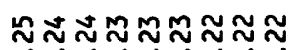
00000000

ํํํํำ 0ं000ं

นึ.

ஸे

000000000

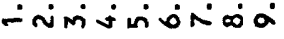

000000 - imivivio

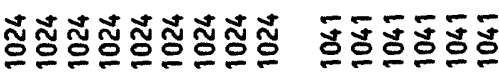

\section{0}

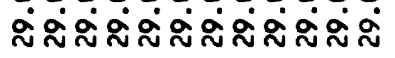

0000000000000

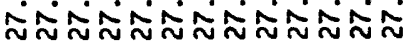

0.0 .000 .000

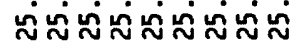

000000 ลั่ง 


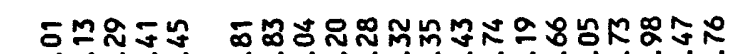

$\infty \omega \infty \omega \infty$ i

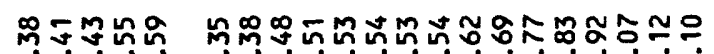

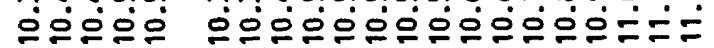

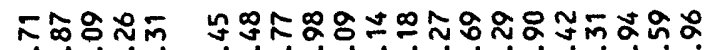

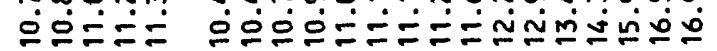
$\dddot{-}$

$\approx \approx \approx \cong ล$ ทำำนก๊

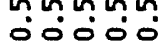

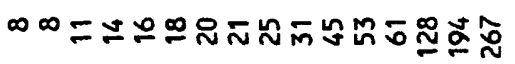

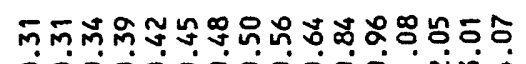

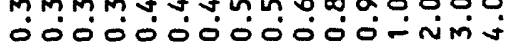

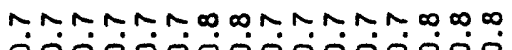

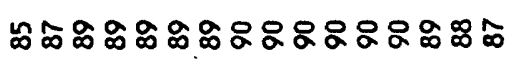

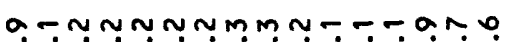
$\infty \dot{a} \alpha \dot{\alpha} \alpha \dot{\alpha} \alpha \dot{\alpha} \alpha \dot{\alpha} \alpha \dot{\alpha} \alpha \dot{\infty} \infty \dot{\infty}$

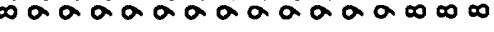

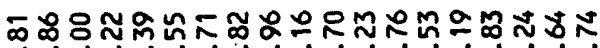

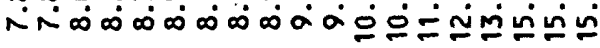

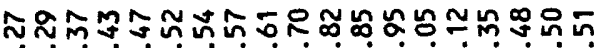

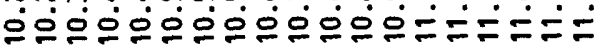

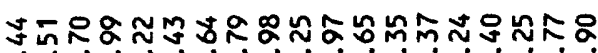

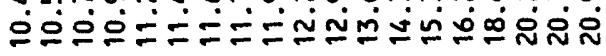

$\stackrel{9}{\circ}$

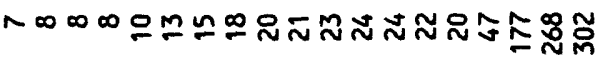

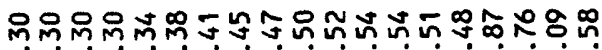
000000000000000

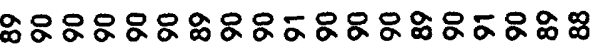

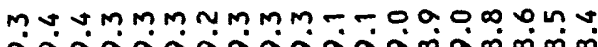
n nna. åa

నิనిన్స

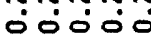

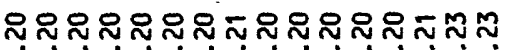

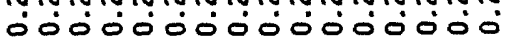

M.M.M.M. . . M.M.M. .

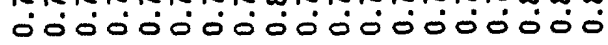

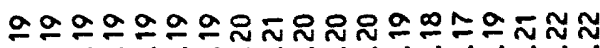
0000000000000000000

0000000000000000000 


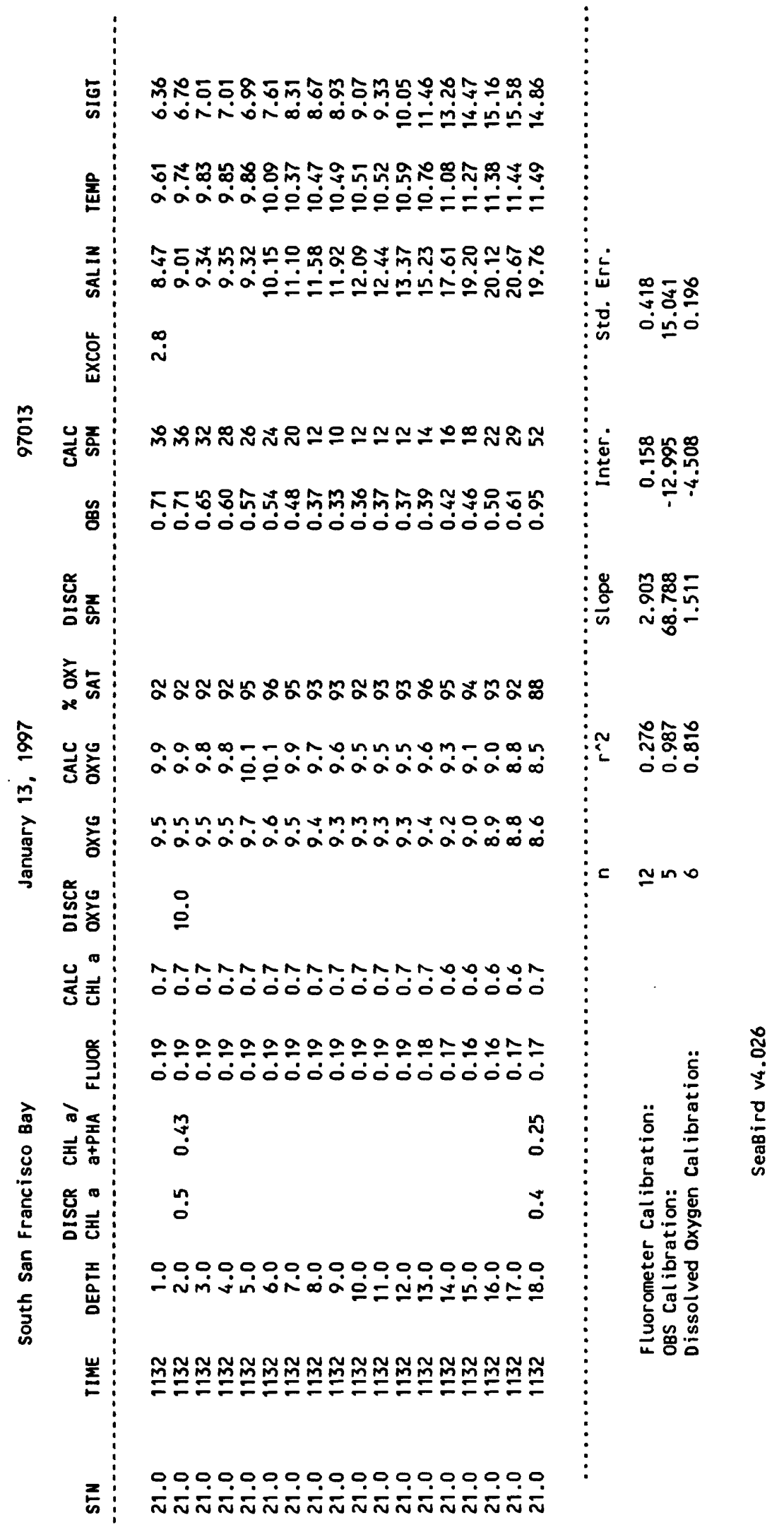




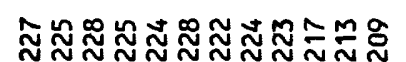

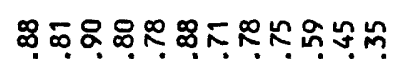

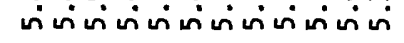

8888888888880

0ं0000000000

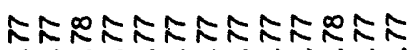
QOODOOOOOOOOO

๖ะะะะะะะะะะะะ ó0000000000
888888888888

๑00000000000

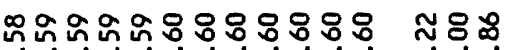

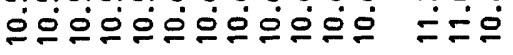

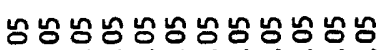
00000000000

ธิธะ๐ 00ं

j

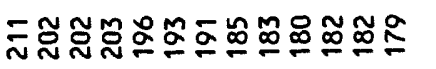

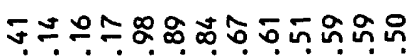

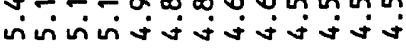

ปें

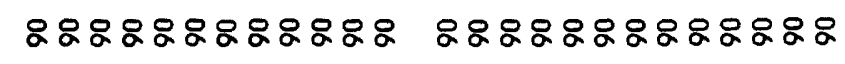

0.0 .0 .0 .0 .0 .0 .0 .09 .9

agagagagagaga.

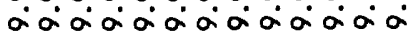

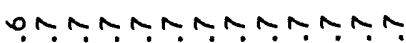

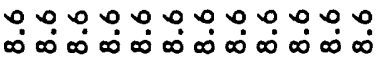
$\infty \infty_{\infty}^{\infty} \infty \dot{\infty} \infty \dot{\infty} \infty \infty_{\infty}^{\infty} \dot{\infty} \infty \dot{\infty} \infty \dot{\infty} \infty$

$$
\hat{a}
$$

$$
\stackrel{\infty}{\infty}
$$

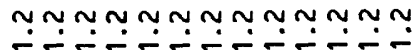

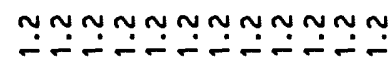

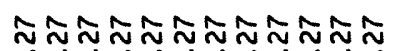
000000000000

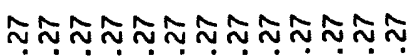
0000000000000

$$
\begin{aligned}
& \text { j. } \\
& \text { ฯ }
\end{aligned}
$$

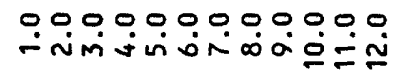

0000000000000

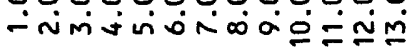

字

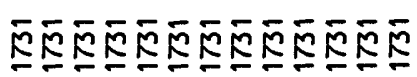

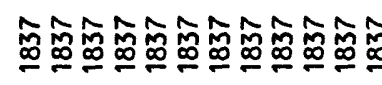

0.09090 .09000

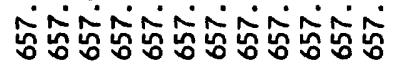

0000000000000

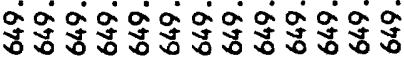

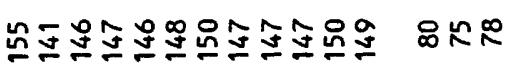

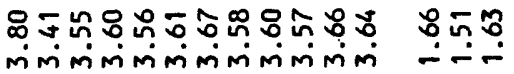

$\stackrel{\alpha}{a}$

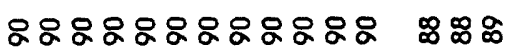

000000000000.010

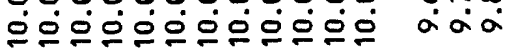

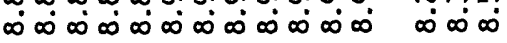
$\stackrel{\circ}{a}$

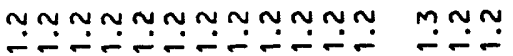

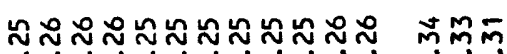

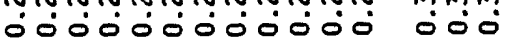

s.

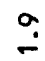

000000000000000

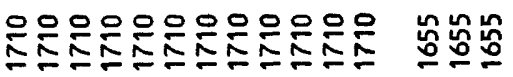

000000000000000 जiñiñNiñNiñin mimm $\infty \infty \infty \infty \infty a, a, a, a, n m n$

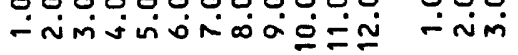


88888888888

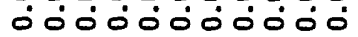

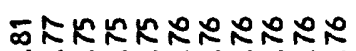
ஹ்ㅇㅇㅇㅇㅇㅇㅛ

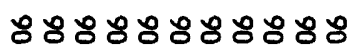

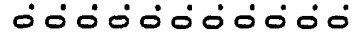

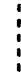

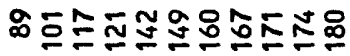

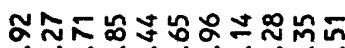
-ñinmivisoj

888888888888888888

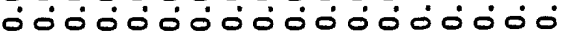

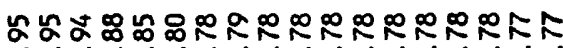

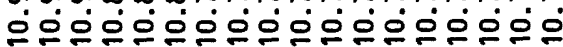

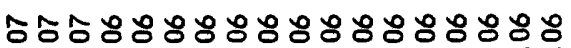
ó000000000000 $\stackrel{\leftrightarrow}{\circ}$

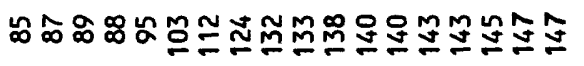
๓ฌฉ -
888888888888

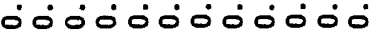

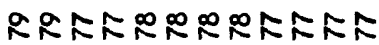

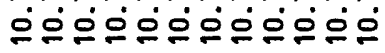

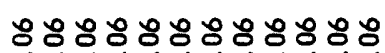
i000000000 $\hat{n}$

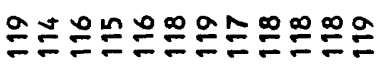

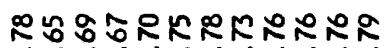
जNंNiñNiñNiñ

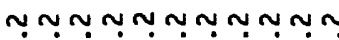
-

ำనని జ 0000000000

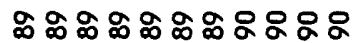

$\infty 0.090 .00000$

$\sim \circ 00000000 N$ $\infty \infty \dot{\infty} \infty \dot{\infty} \infty \dot{\infty} \infty \dot{\infty} \infty \dot{\infty} \infty \dot{\infty}$

总
00000000000

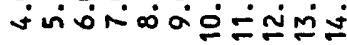

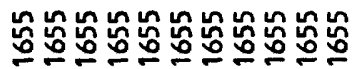

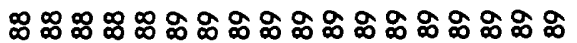

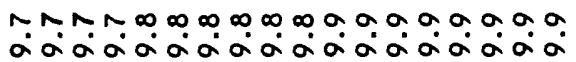

mmmutum n n m n n

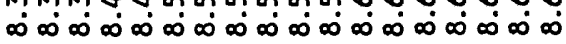

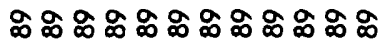

$\infty \infty, \infty, \infty \infty \infty, 0, a$

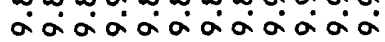

un un in in in in 0000 $\infty \infty \infty \infty \infty \infty \infty \infty \infty \infty \infty \infty \dot{\infty}$
mMก M-R-

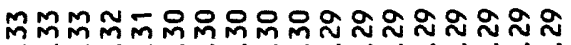
0000000000000000

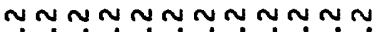

$\bar{m} \bar{m} \bar{m} \bar{m} \bar{m} \bar{m} \bar{m} \bar{m} \bar{m} \tilde{m}$ 0000000000
000000000000000000

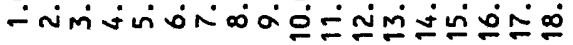

000000000000

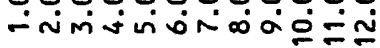

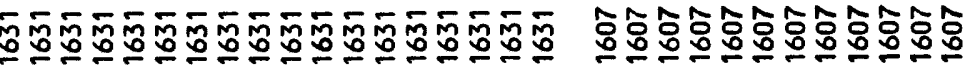

000000000000000000

000000000000

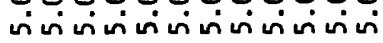


8.888888888888

- 00000000000

ก.

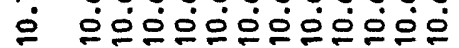

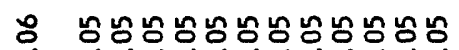

- 00000000000

$\bar{\infty}$

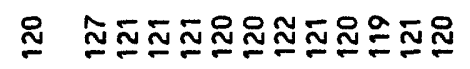

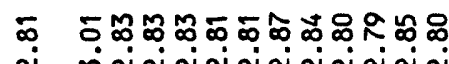

i miñininivinin

ì

各高

స్

ड़

总 중

这퐁

:

$\infty$

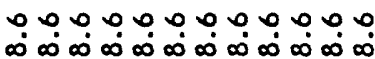

$\stackrel{0}{\circ}$

צִ

ஊே

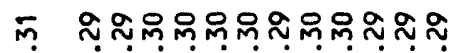

- 00000000000

m.

공

=

点

000000000000

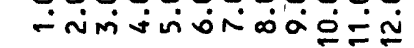

0000000000000000

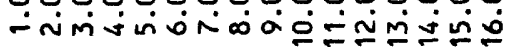

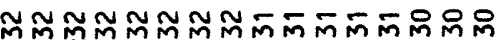
000000000000000

ติMmติ 0000000000

$\stackrel{?}{0}$

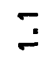

崖

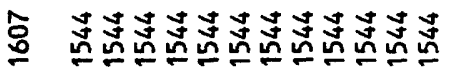

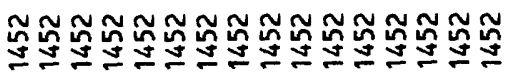

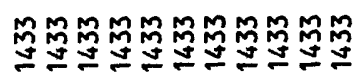

00000000000 - iminioná

z

0000000000000000

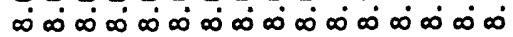
웅영여영영영 


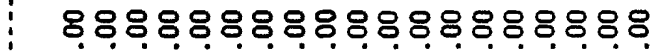
0000000000000000

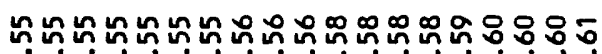

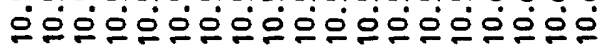

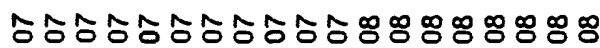
ऽं0ं0000000000000
88888888888888888888

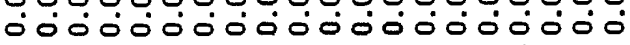

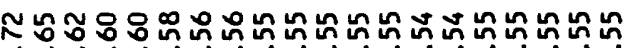

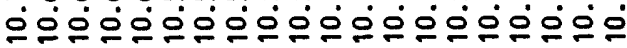

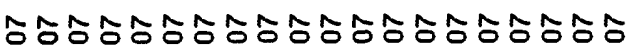
ó0000000000000000

$\bar{a}$

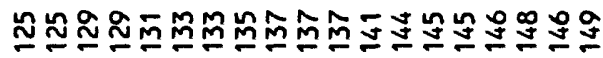

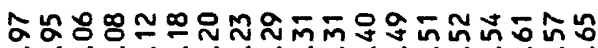

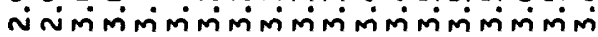

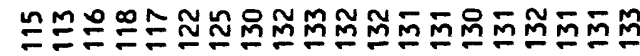

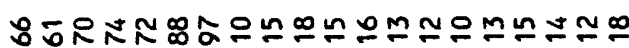
NiñNiñinmmimimimimimimimi $\overline{0}$

88

요 $\therefore \dot{0}$

\&̊ㅇ․ $\dot{0}$

$\stackrel{n}{\approx} \stackrel{0}{\circ}$

$8: 0$

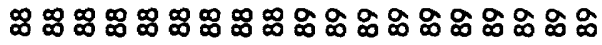

$\infty \infty \infty \infty \infty \infty \infty \infty \infty \infty \infty \infty \infty \infty \infty \infty \sigma a \sigma$

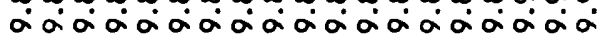

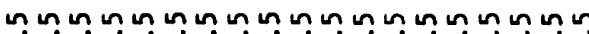
$\infty \infty_{\infty}^{\infty} \infty \infty_{\infty}^{\infty} \infty \infty_{\infty}^{\infty} \infty \infty_{\infty}^{\infty} \infty \infty_{\infty}^{\infty} \infty \infty_{\infty}^{\infty} \infty \infty^{0}$

芯임

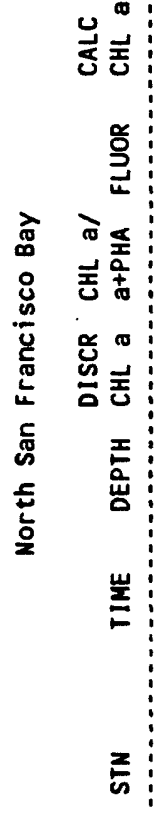

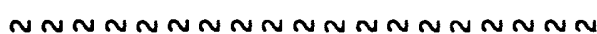

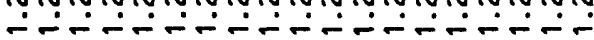

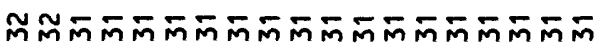
๑000000000000000000
0000000000000000000

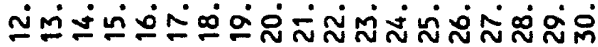

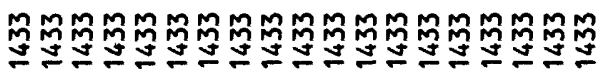

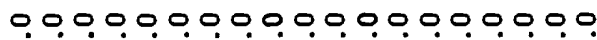

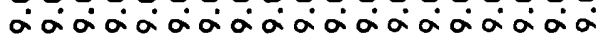

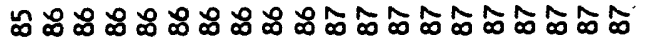

曲

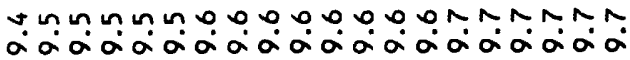

a00000-7. N N N N NNMMM

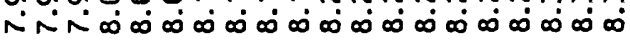

$\tilde{a} \tilde{a}$

$m_{\infty}^{m}$
N $M N$ MN

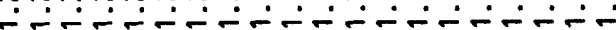

$\dddot{\Upsilon 0}$

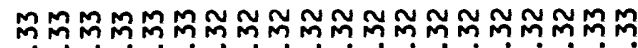

m.m. 00000000000000000

00
00000000000000000000

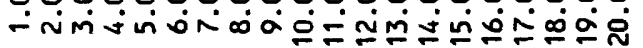

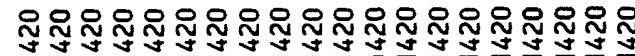




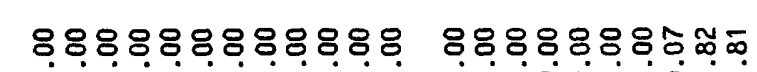

000000000000

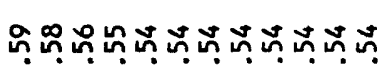
웅ㅇㅇㅇㅇㅇㅇㅇㅇㅇㅇ

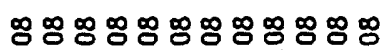
-00000000000

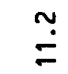

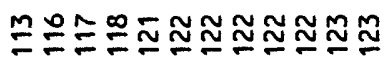

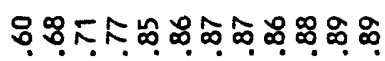

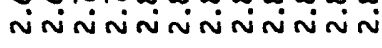

总

各高

స్

㝴

ก.ก $\infty \infty \infty \infty \infty \infty \infty \infty \infty \infty \infty \infty$

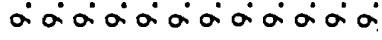

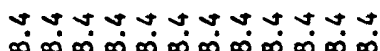
$\infty \infty \infty \infty \infty \infty \infty \infty \infty \infty \infty \infty \infty$

递

옳롱

울

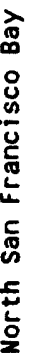

Mบบบบกบกกบก

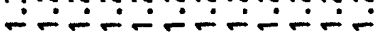

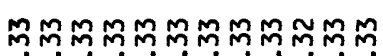

ơơơơơơ

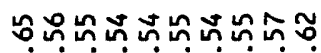

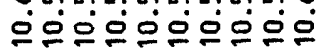

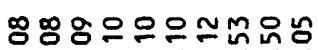

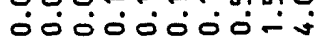

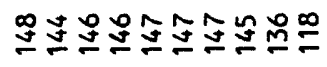

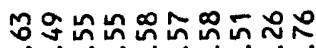

miniminiminimín

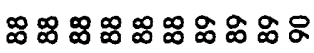

$\infty \infty \infty, \infty \infty \infty \infty$,

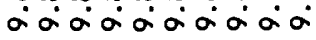

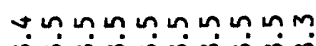
$\infty \infty \infty \infty \infty \dot{\infty} \infty \dot{\infty} \infty \dot{\infty} \infty \dot{\infty}$

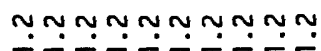

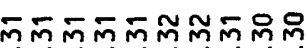
000000000

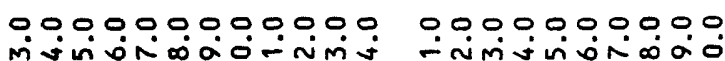

$\stackrel{+}{a}$

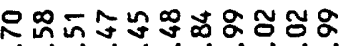

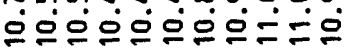

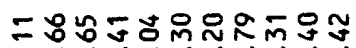

ธั口-

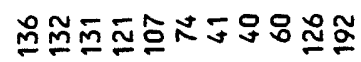

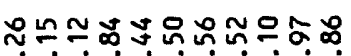

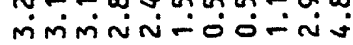

$\stackrel{\circ}{\dddot{0}}$

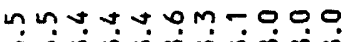

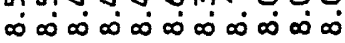

$\stackrel{\circ}{\circ}$

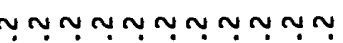



บก $ง ก \sim ก$

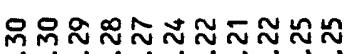
0.000000000

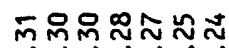<smiles>[Ca]=[Hg]</smiles>

$\ddot{\circ}$

$\stackrel{m}{\circ}$

$\stackrel{\infty}{\circ}$

00000000000

-nimino

0000000

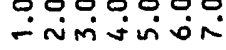

崖

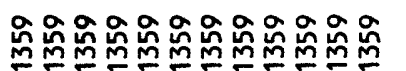

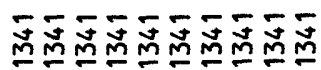

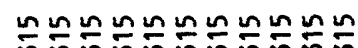

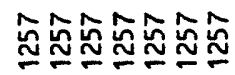

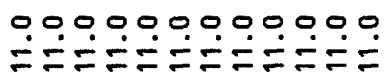

0000000000

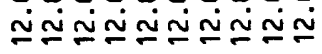

00000000000 ต்ற்ற்ற்ற்ற்ற்ற்

0000000

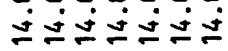




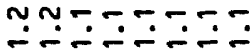

ㅊํ요유응

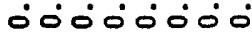

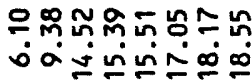

ก⿻ำ

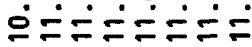

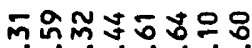

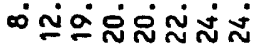

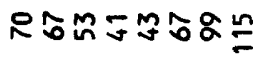

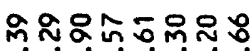
- To0j-nin

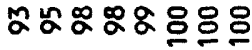

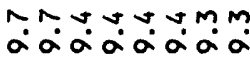

tmom

moñintin

$\stackrel{\circ}{\circ}$

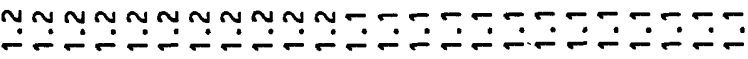

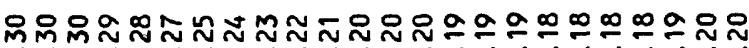
000000000000000000000

$$
\text { กั }
$$$$
\stackrel{\circ}{-}
$$

.

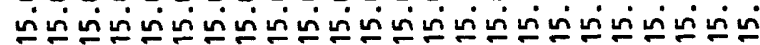

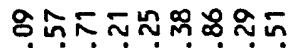

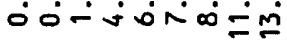

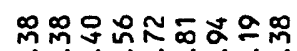

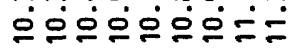

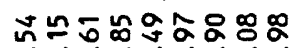
○-

a.

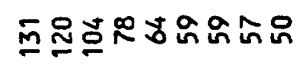

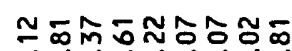

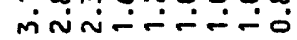

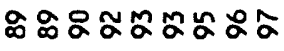

aagonN

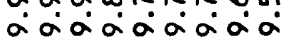

$000 n m m \sim-a$ $\infty \infty_{\infty} \dot{\infty} \infty \dot{\infty} \infty \dot{\infty}$

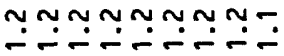

ลูกลกลำกำ व0ं00000

0.0000000 - imivioñó

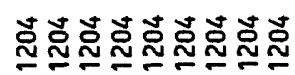

000000000

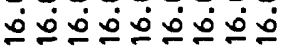




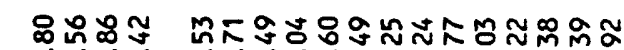
옹ำ

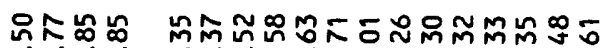
立亡

ธ유웅 ลำ่

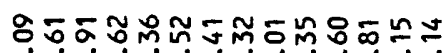
$\stackrel{0}{a}$

0.009.9.9.9.0.0.0.0\%

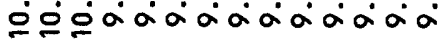

\section{anon}

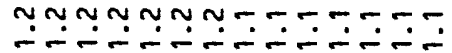

$\stackrel{\infty}{\simeq} \frac{a}{9}$

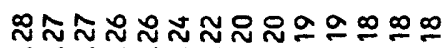
$\therefore \circ 0^{\circ}$ 0000000000000

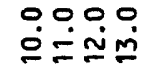

00000000000000

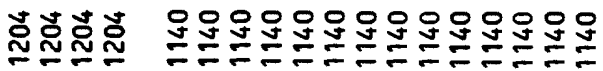

000000000000000000

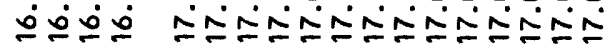

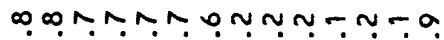
$\infty \infty_{\infty}^{\infty} \infty \infty_{\infty}^{\infty} \infty \infty_{\infty}^{\infty} \infty \infty_{\infty}^{\infty} \infty \dot{\infty}$

$\stackrel{\circ}{\circ}$

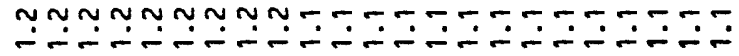

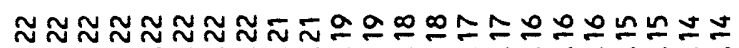

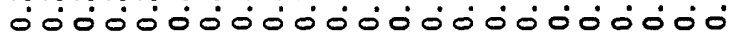
๘
$\dot{0}$ $\stackrel{\infty}{0}$

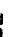

00000000000000000000000 -nmง

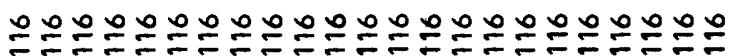

00000000000000000000000

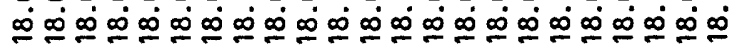

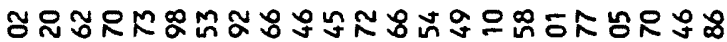

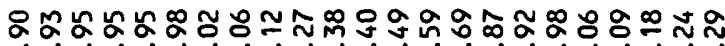

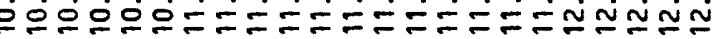

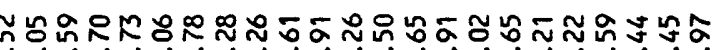

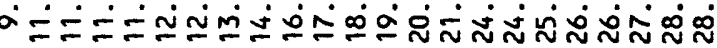
$\therefore$

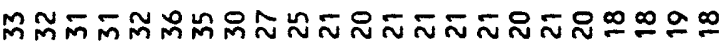

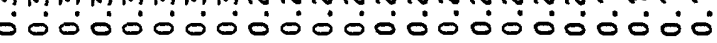

$\tilde{n}$

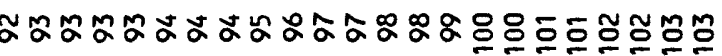

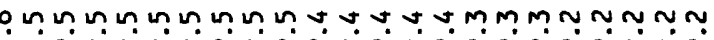




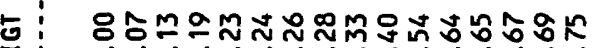

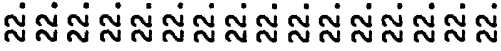

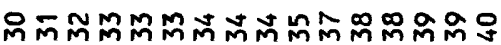

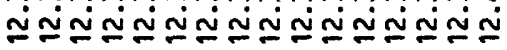

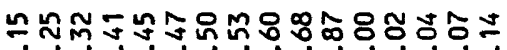

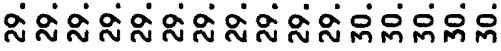

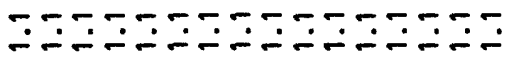

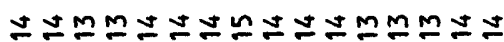
व0ं0ं0ं00000000

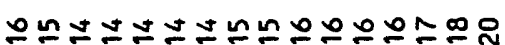
0ं00ं000000000

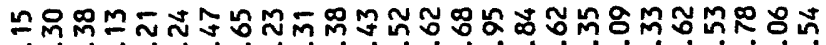

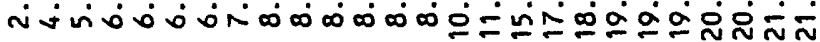

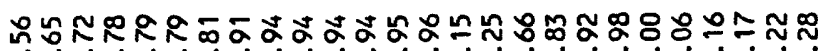

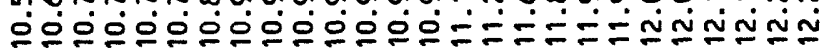

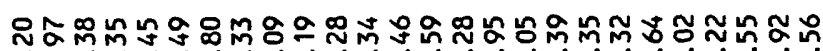

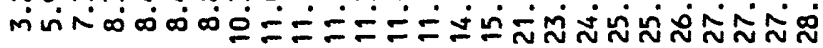

$\stackrel{\circ}{n}$

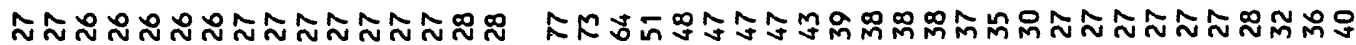

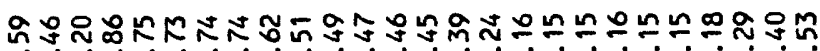
- 过0000000000000000000

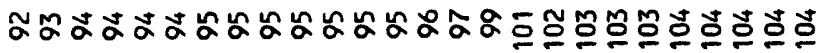

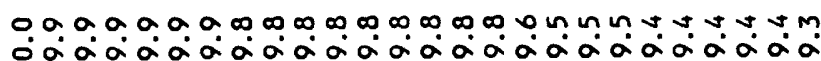

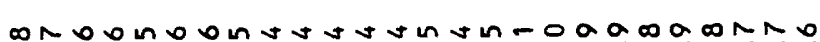

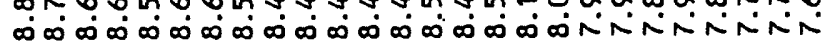

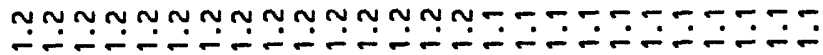

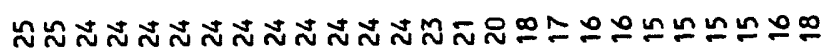

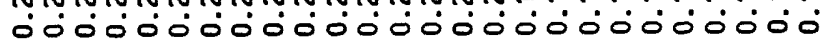

0000000000000000

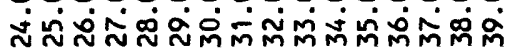

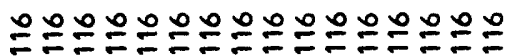

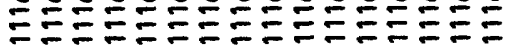

0000000000000000

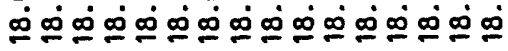

00000000000000000000000000 -

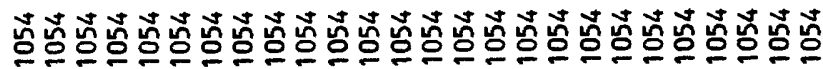

00000000000000000000000000

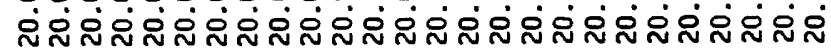




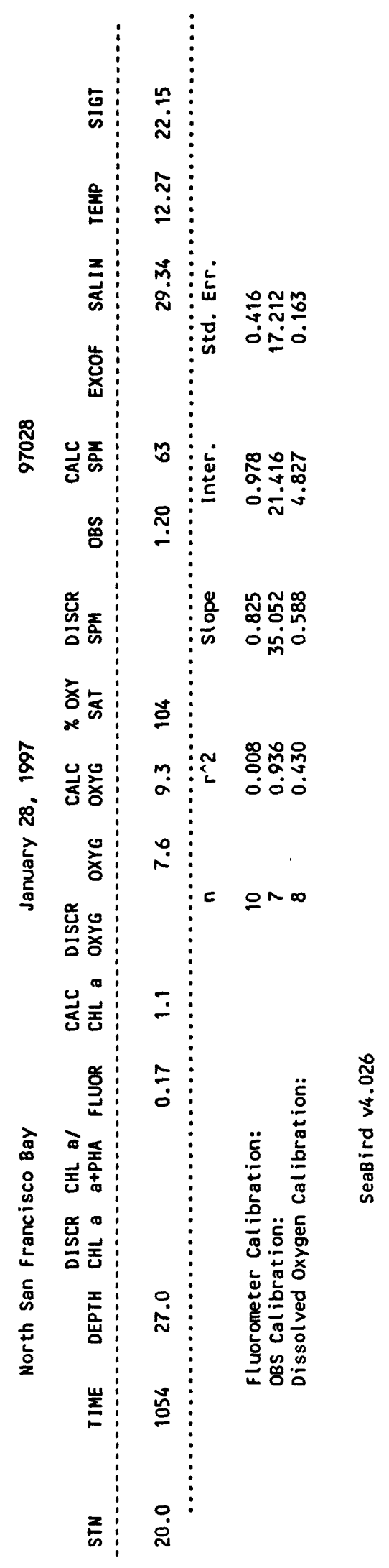




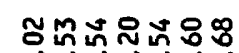

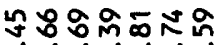

inin $\infty \infty_{\infty}^{\infty} \infty$

ㅇำกำ

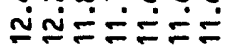

오ำำำ

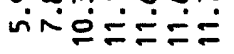

$\therefore$ - iminio

우ำํำํำำำ

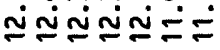

๓ึษ์ธิ์ำณ

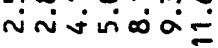

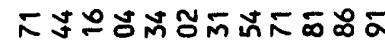

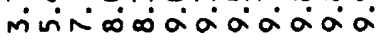

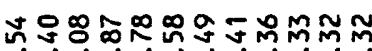

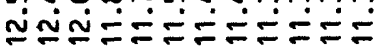

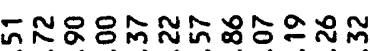

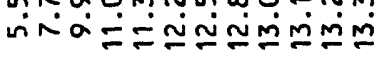

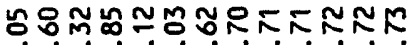

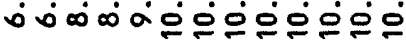

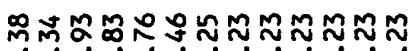

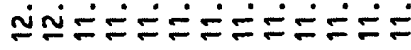

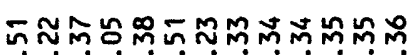

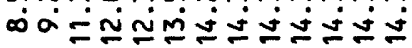

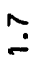

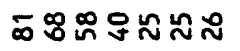

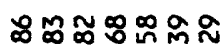

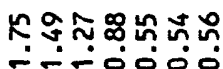

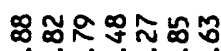
$\because-\because \dddot{0}$

å.

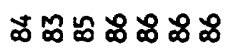

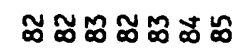

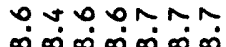

مீ

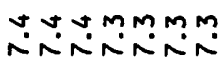

$$
\stackrel{\infty}{\infty}
$$

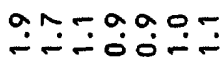

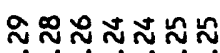
व.00000

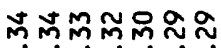
0000000

$000,00 \infty \infty$ miñiñi? $\dot{0}$

0.0 .0000 - nimivion

0000000 - imivivion

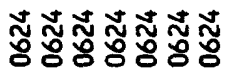

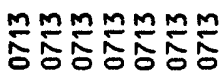

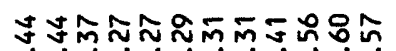
000000000000

$\stackrel{0}{n}$

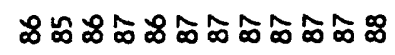

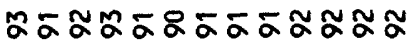

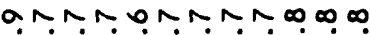
$\infty \infty_{\infty}^{\infty} \infty \infty_{\infty}^{\infty} \infty \infty_{\infty}^{\infty} \infty \infty_{\infty}^{\infty} \infty \infty_{\infty}^{\infty} \infty \infty_{\infty}^{\infty}$

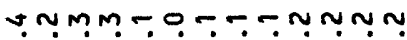

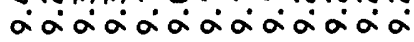

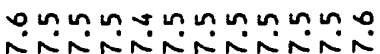

ம

$\stackrel{\infty}{\infty}$

$\dddot{\sim}$

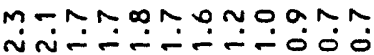

TONonoy tom onn minini-

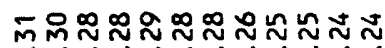
0000000000

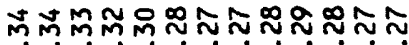
0000000000000$$
\text { á. }
$$

$\stackrel{\circ}{\circ} \stackrel{2}{0}$

$\stackrel{0}{0}$

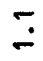

$\stackrel{\sim}{-}$

000000000000 -

0000000000000 -

\section{崖}

00000000000000

른

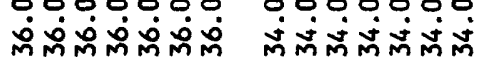

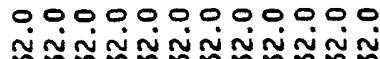

0000000000000

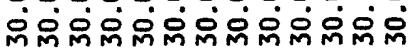




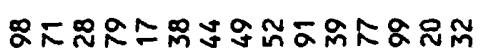

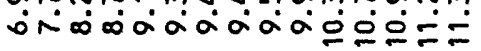

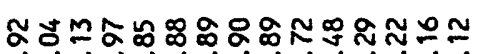

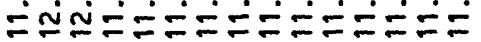

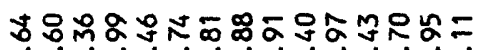

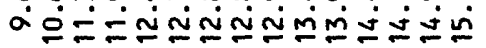

$\Xi$

芯

害紊

\%ั

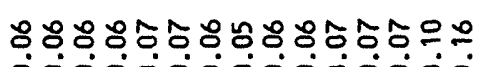

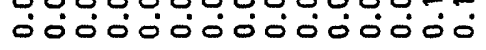

$M M M M M M M M M M M M M N N$

nnwnme-nummo

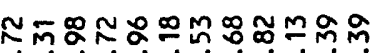

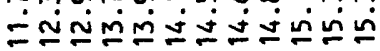

$\stackrel{9}{\circ}$

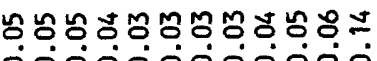

व000000000

$\ddot{n}$

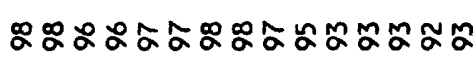

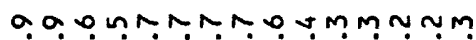

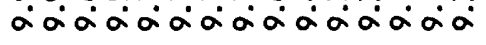

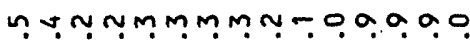

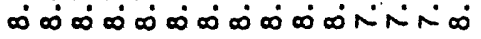

$\infty$

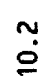

范志

Gmo-Toa-my amo-

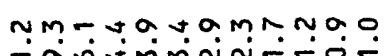

o óvimimiojumiñNa

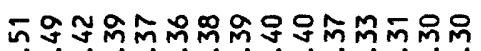

ல00000000000000

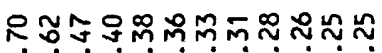
00000000000

$\stackrel{m}{\circ}$

$\stackrel{\circ}{\circ}$

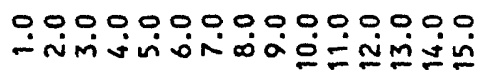

000000000000

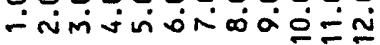

00000000 - invisionio

ํํำํํํํํํำำำ 00000000

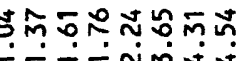

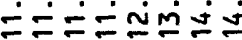

$\Xi$

JMmMmmNm

$0 \infty \infty \infty N$

$\stackrel{2}{2} \simeq$ 00000

m.

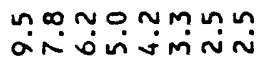

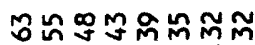
o0000000

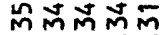

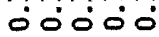

$\stackrel{\circ}{\circ}$

$\hat{m}$

00000 -nimisin

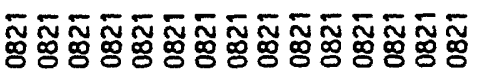

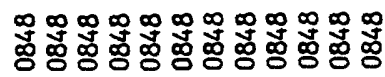

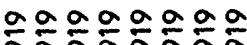

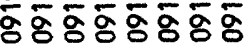

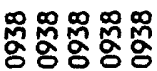

000000000000000

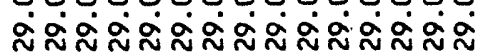

0.00000000000.

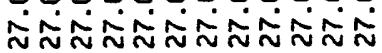

0.000 .000 .0

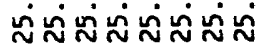

0.0 .0 .00

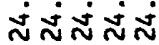




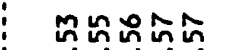
ㅇํㅇㅇำ

กฺฺฺุ̣̣̣

モニニニニ

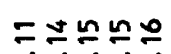

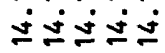

茁

:

爮

$\circ 0000$

$m \simeq \simeq M \pm$ वं००००

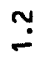

ที่ธ์

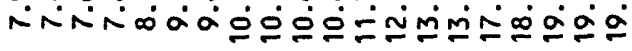

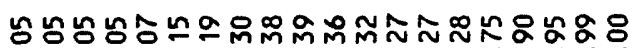

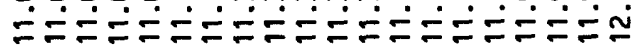

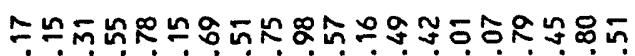

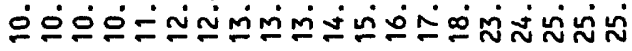

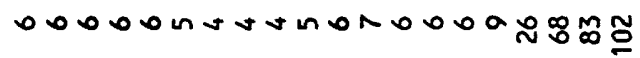

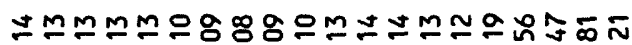

o0000000000000

จุญ ำก

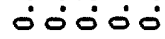

崖

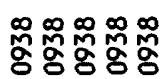

0.000 .0 ก่งก่ง

i.

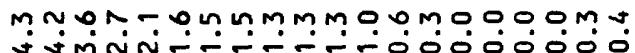

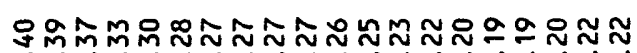

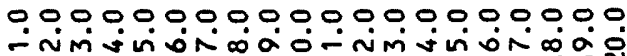
00000000000000000
$0.00000000000,00000,000$

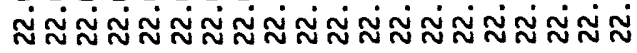

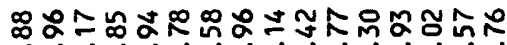

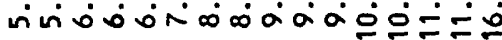

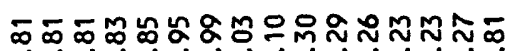

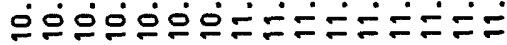

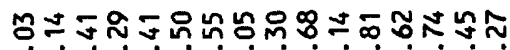

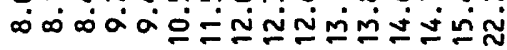

$\stackrel{n}{\sim}$

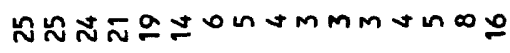

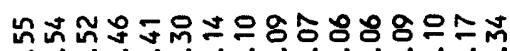
000000000000000

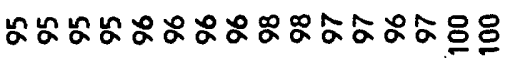

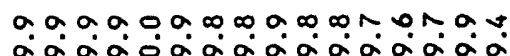
nn

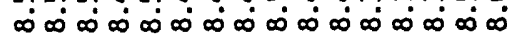

$\ddot{a}$

กับน์

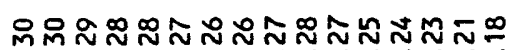

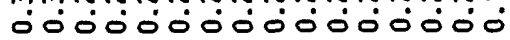

$\infty$

$\check{-}$

0000000000000000

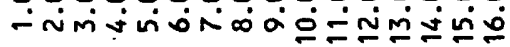

\section{음응응응응응응응응응응응응응응응응응응은}

జ్రీ:

0.000 .0000000000000 ஸ்亠幺ं் 


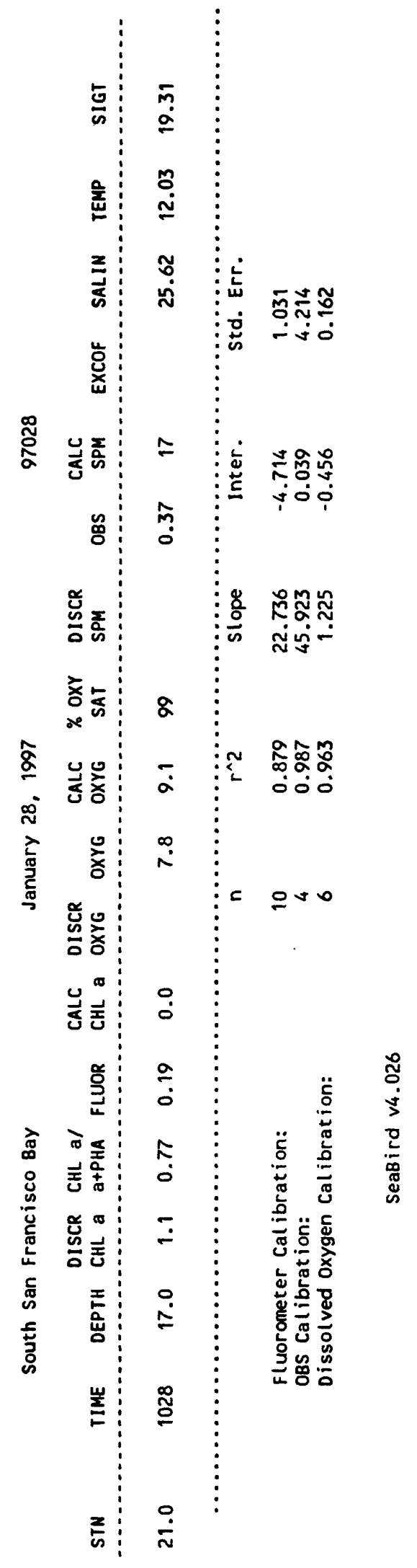




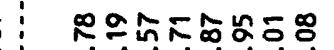
ivinivinisio

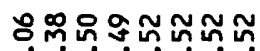
ํํำํำ

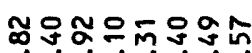

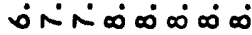

$\hat{\sim}$

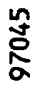

远

ํㅜㄴ

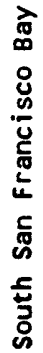

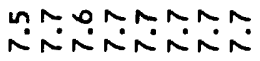

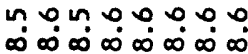

$\dddot{n}$

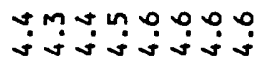
$\begin{array}{ll}\tilde{0} & \tilde{n} \\ \tilde{m} & \tilde{o} \\ \tilde{m} & \hat{m}\end{array}$ in

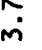

0.000000

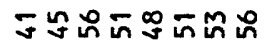

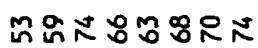
O0.00000

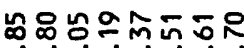

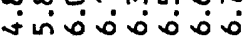

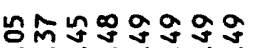

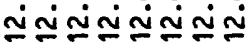

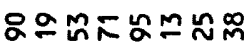

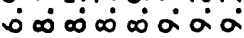

$\stackrel{\infty}{\sim}$

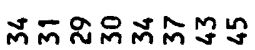

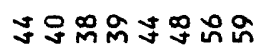
क0000000
ㅅํำำ in

뜨ำกิన̄

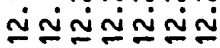

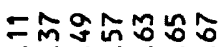
$\infty \infty \infty \infty \infty \infty$

$\stackrel{n}{m}$

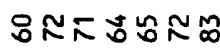

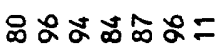
00000:

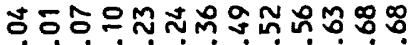
ด.

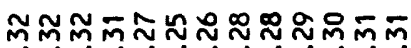

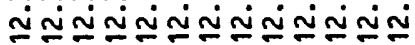

家品 또ำ

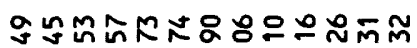

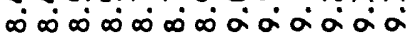

$\mathscr{8} \approx \approx$ $\infty \infty 0^{\circ}$

$\stackrel{0}{\sim}$

$\stackrel{m}{i}$

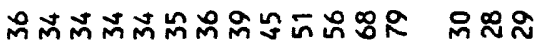

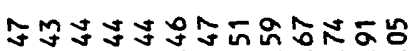
क000000000

minn :0ं

$$
\dddot{n}
$$

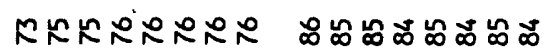

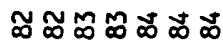

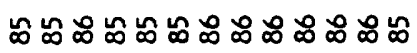

๓

mִ

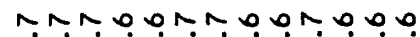
0.9 $\infty \dot{\infty} \infty \dot{\infty} \infty \dot{\infty} \infty \dot{\infty} \infty$

- ก บ บ บ ก

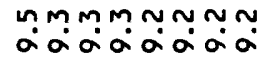

$-\infty \sim \infty-\infty \min$

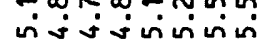

P.o.t.Tn $\infty \infty \infty \infty \infty \infty \infty \infty \infty \infty \infty \infty \infty \infty \infty)$ $\infty \dot{\infty} \infty$

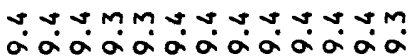
웅ํำ $\tilde{\infty}$

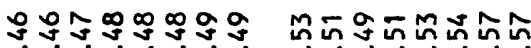
0000000 0000000

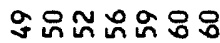

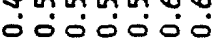

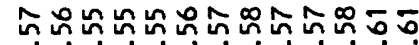
000000000000

กิกฺำ

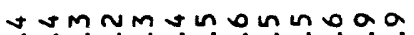
मrivinuming

un o nini

$$
\dot{0}
$$

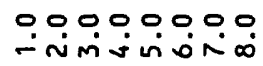

0.00000

0000000000000

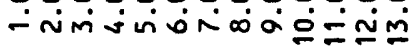

웅울

$\stackrel{0}{n}$

in

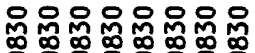 \\ นิ๊}

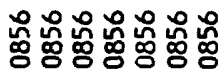

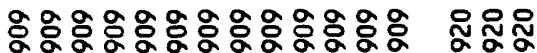

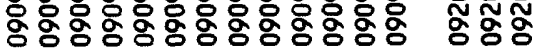




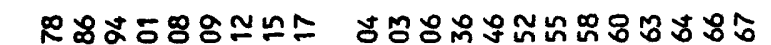

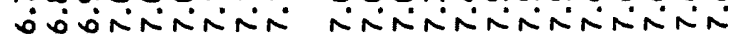

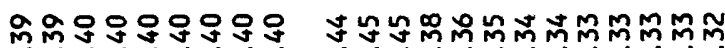

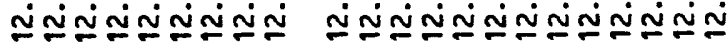

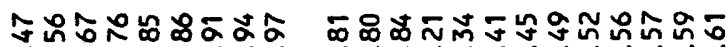

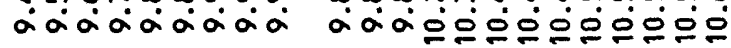

$\stackrel{\circ}{i}$

ฉิ์

趆高

号

苟

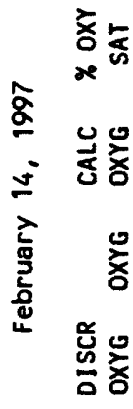

똥롱

ที⿻上丨

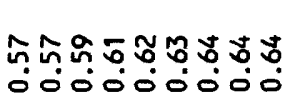

ֻே

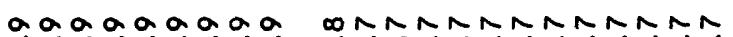
$\infty \infty \infty \infty \infty \infty \infty \infty \infty \infty \infty \infty^{\circ}$

$0 \% 0 \%$ :

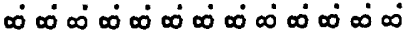

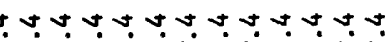

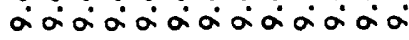
aरajajasa $\stackrel{2}{\mathfrak{0}}$

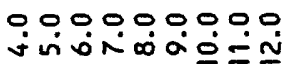

0000000000000

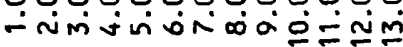

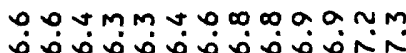

D 0ं00000000000

؛ִ

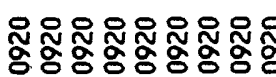

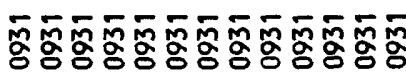

000000000

0.00000000000

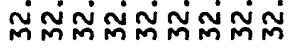

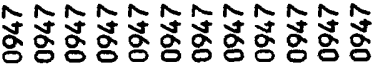

응요용음

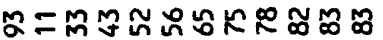

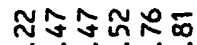

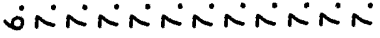

intivin

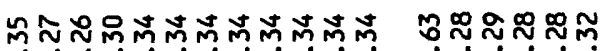

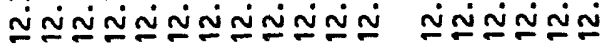

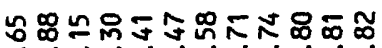

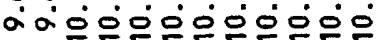

gmm중중 응ㅇㅇㅇㅇ

$\stackrel{+}{-}$

$\stackrel{+}{\check{2}}$

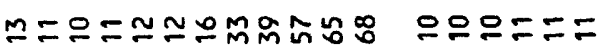

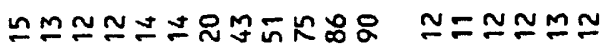

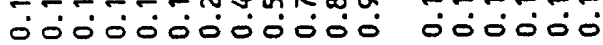

$\tilde{\infty}$

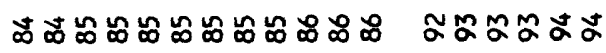

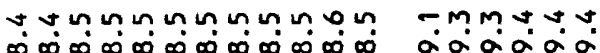

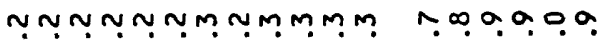

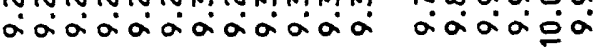

$\bar{a}$

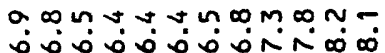

Noon in ด.

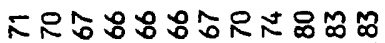

SRกลกั: O0ं000000000 Oं0ல0ல0

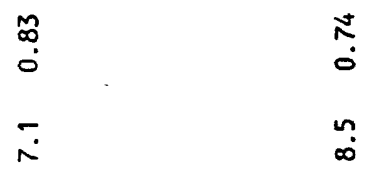

000000000000

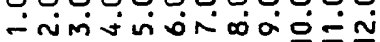

000000 - imivisio

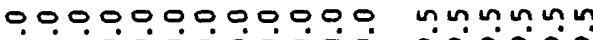

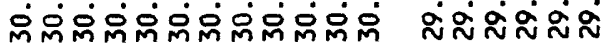




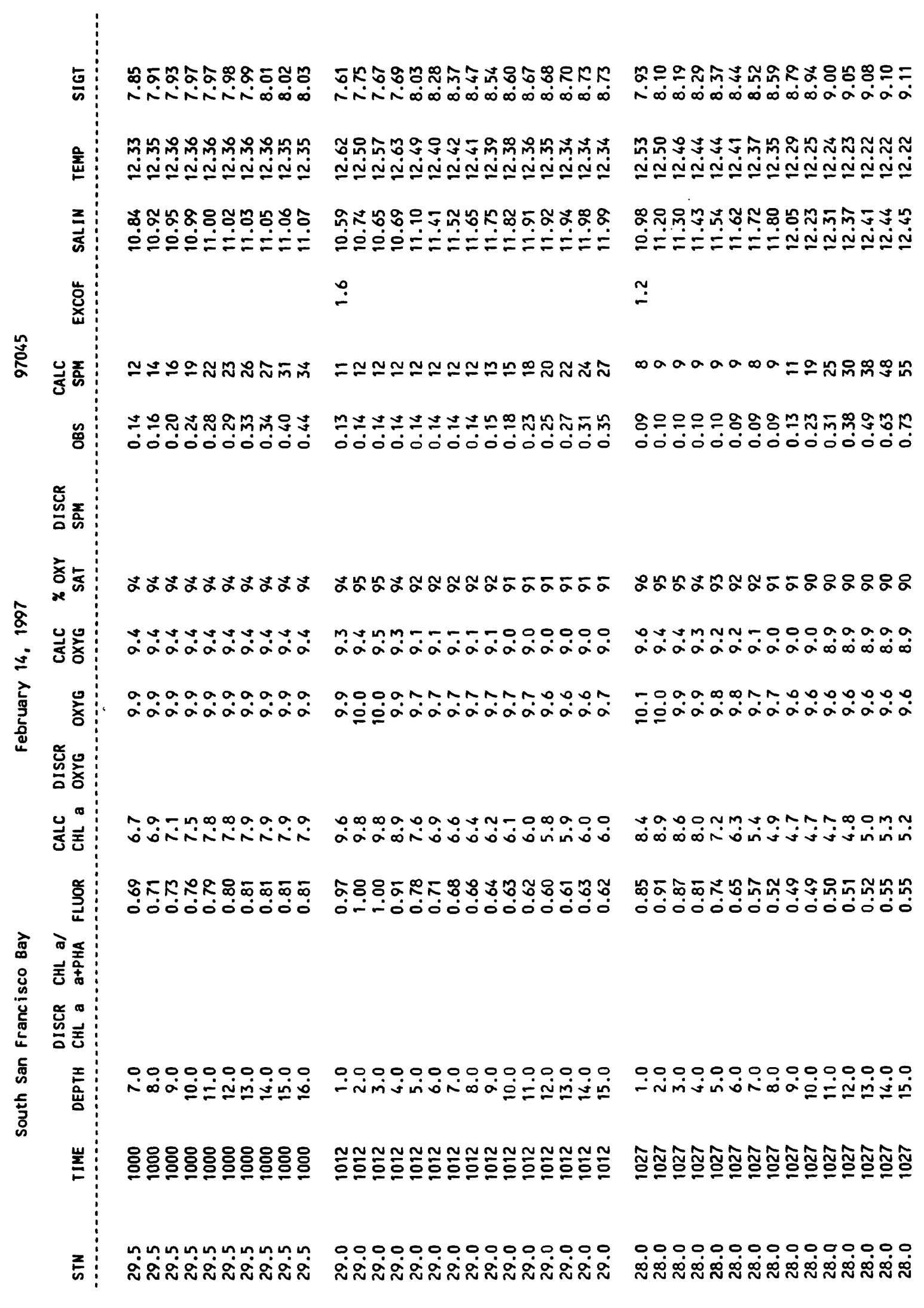




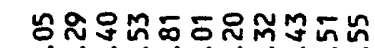

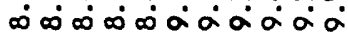

テำำำกำำำำำ

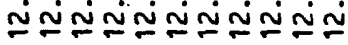

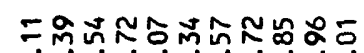

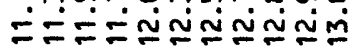

$\because$

รั้

这

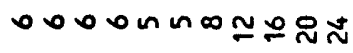

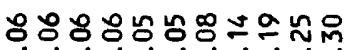

00000000.

$\hat{n}$

蒙

ชิ้

$\underline{\underline{a}}$

$\pm$

㝴艺

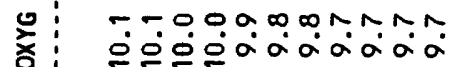

늠

㝴至

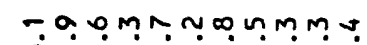

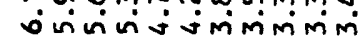

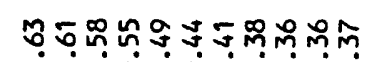

00000000000

$\mathfrak{n}$

ڤ̊.

폰 京

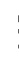

톯

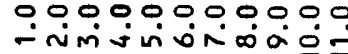

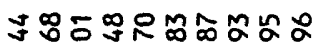

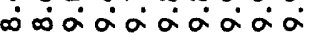

ติ

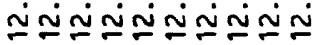

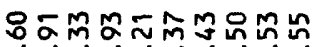

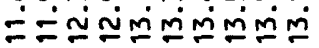

$\stackrel{5}{\circ}$

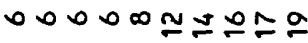

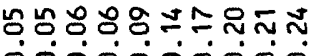
0000000000

끙쭝ㅇㅇㅇㅇㅇㅇㅇㅡ 00000000

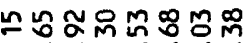

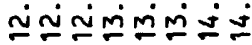

$\stackrel{9}{-}$

S I U O

$m m \checkmark \infty \infty a=m \pi$

ำํํㅇํํㅁำำำก 000000000

$\stackrel{9}{s}$

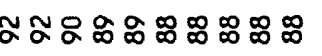

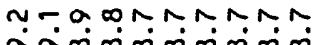

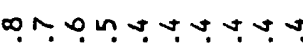

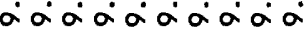

åaำ

?.0.9.0.?.?.

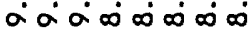

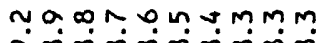

$\infty \infty \infty \infty \infty \infty \infty \infty \infty \infty$

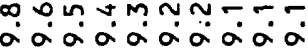

m.

임ำ

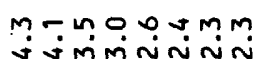

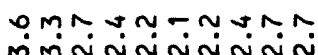

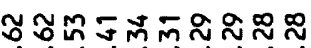
00000000

ำ ำm 0000000

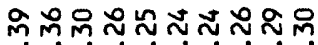
000000000

迎

$\stackrel{m}{n}$

$\stackrel{\infty}{\sim}$

$\stackrel{\infty}{\sim}$

0000000000

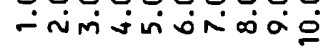

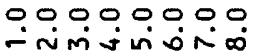

0000000000

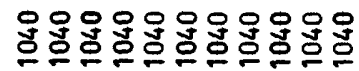

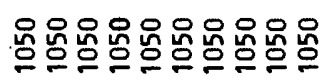

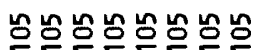

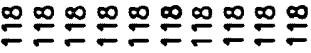

0.0000000000

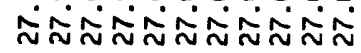

0.000 .00000

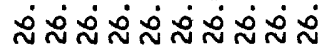

$0,0,0,0,00$

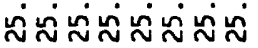

0.000 .000 .000$.

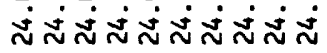




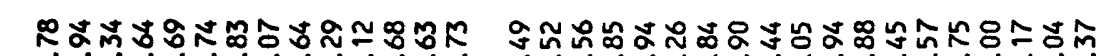

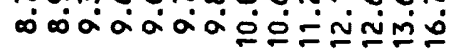

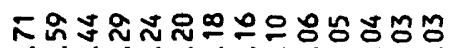

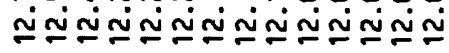

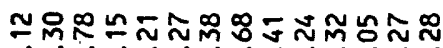

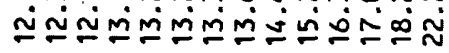

$$
\hat{0}
$$

TMOAJOOMNNNMMm rivismininininiñiñ

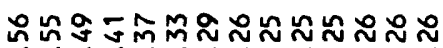

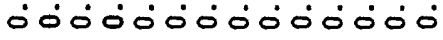

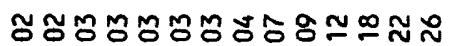
O000000000000

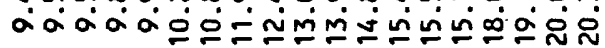

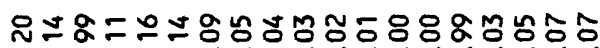

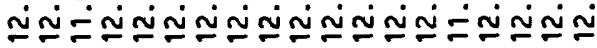

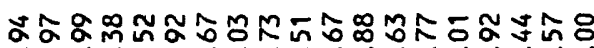

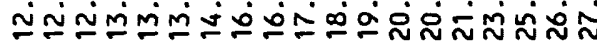
$\stackrel{\circ}{\circ}$

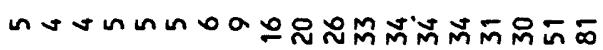

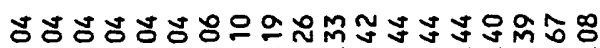
00000000000000000-
ถัปปกณะะก $\cos \alpha \cos$

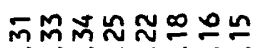

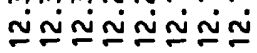

ำณテธณฒ

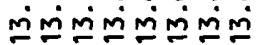

$\hat{0}$

$\ln \sin \min \min \sin$

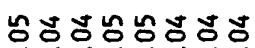
00000000

in

ఐ

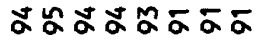

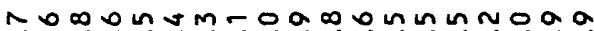
क

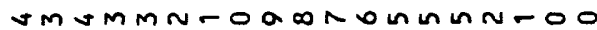

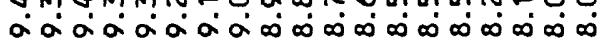

mmon mimininininininiñ

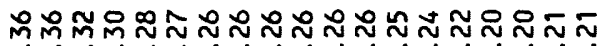

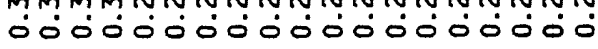

ษ ำ ํำ 00000000

$$
\begin{aligned}
& \stackrel{0}{0} \\
& 0 \\
& 0 \\
& \dot{0}
\end{aligned}
$$

00000000000000

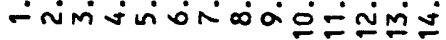

0000000000000000000

-

0.0000 .000 - imivivion

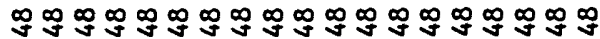

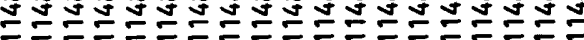

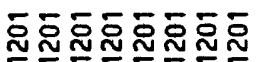




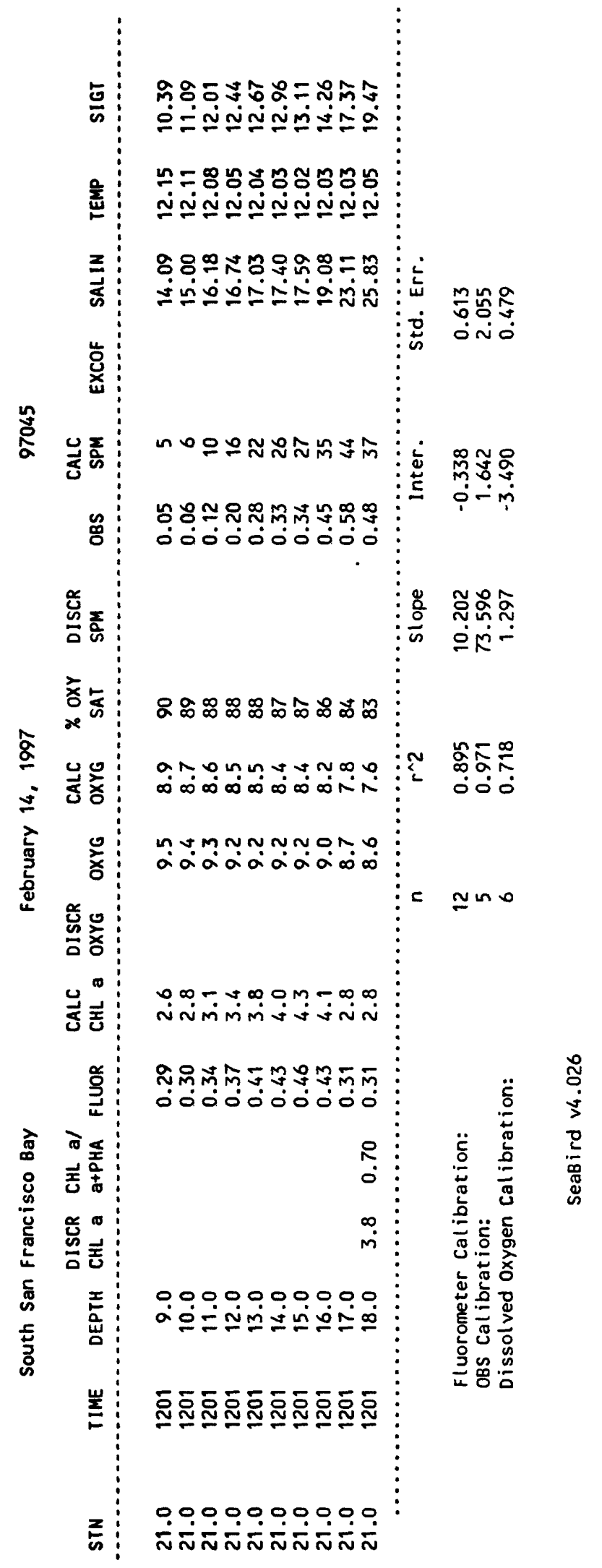




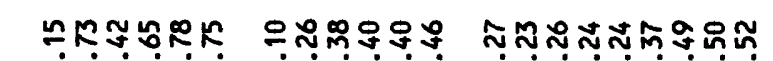

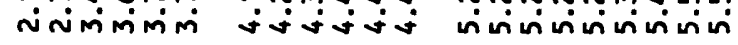

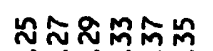

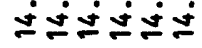

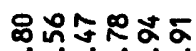

mivivinini

MMM믄

$\dot{q} \dot{ \pm} \dot{ \pm}$

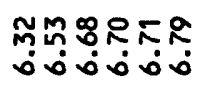

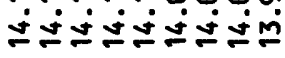

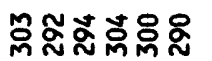

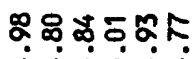

䒴

흠

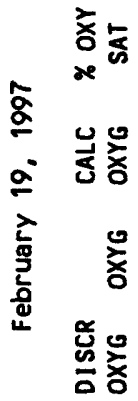

迆퐁

\section{TOMamn}

ํำำธ

ำกำำก

?.

$\stackrel{m}{\infty} \stackrel{+}{0}$

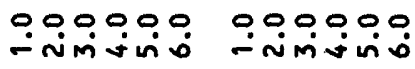

ginnmon

ต்ะேำำ

ษงษำชำ

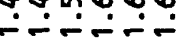

ํํํㅀㅀำ

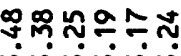

minminimin

$m+4000$

?. 0.9 .90

$\infty \dot{\infty} \dot{\infty} \infty \dot{\infty}$

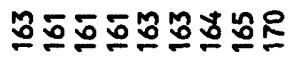

ธํํํํํํํำ

ininininin

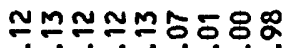

ณญㅁำ

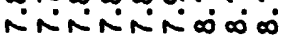

M

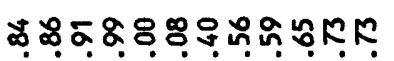

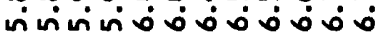

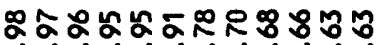

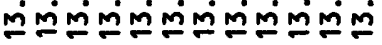

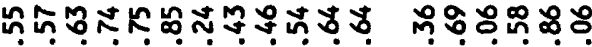

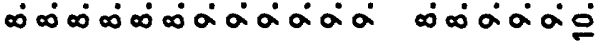

$\hat{m}$

$\dot{i}$

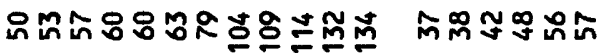

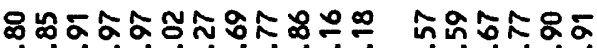
00000ㅁำn 000000

in

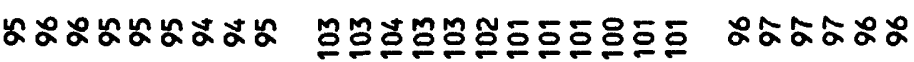

mmmmmmñm åajajaja

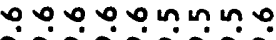
acaiacaia

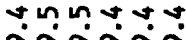

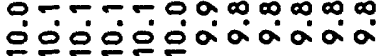

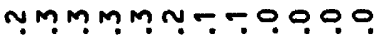
옹ㅇㅇㅇㅇㅇㅇㅇㅇ

A.T.M.?. åáa $a \dot{\alpha}$

$\stackrel{n}{a}$

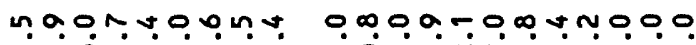

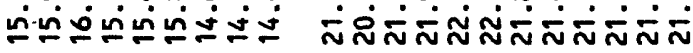

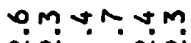

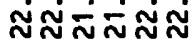

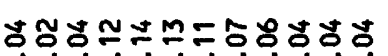
ininininininiñ

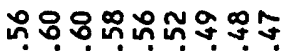

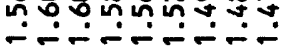

뜨ํㅇㅇํำ inininin

$\stackrel{\ddot{m}}{0}$

$\stackrel{n}{n}$

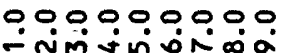

000000000000

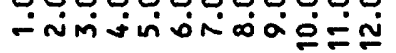

웅ํํ용 


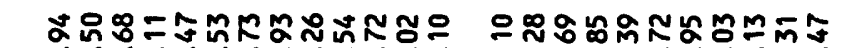

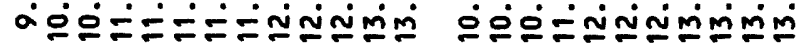

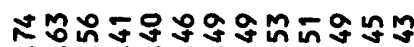

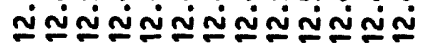

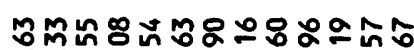

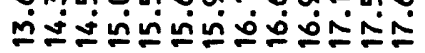

$\dddot{m}$

号

爮

RND

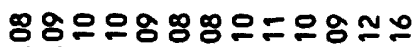

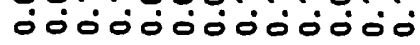

$\stackrel{a}{a}$

各芯

$\underline{\mathrm{g}}$

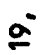

ปูำ

농

芯新

迅롱

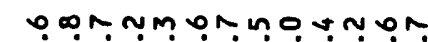

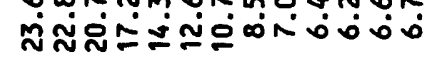

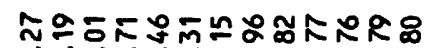

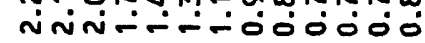

$$
\infty
$$

กั่

$\stackrel{\infty}{\sim}$

$\tilde{\infty}$

픔

0000000000000

满m

놀

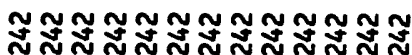

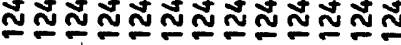

0000000000000

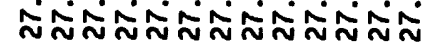

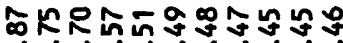

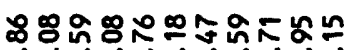

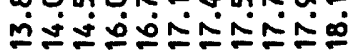

$\dddot{-}$

O

충송옹송영영영용으 ơं0ं000000

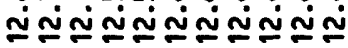

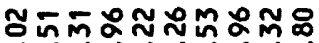

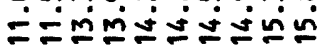

ำนำำกำกิกิ

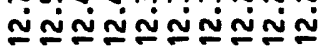

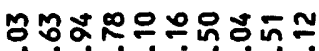

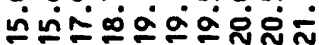

$\because$

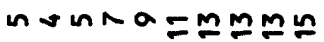

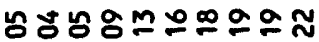
o00000000

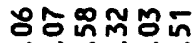

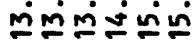

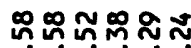
บญํํำ

กระกำก ํㅃㅇํำ

$\stackrel{\infty}{\circ}$

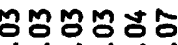
ic000

$$
\stackrel{\infty}{j}
$$

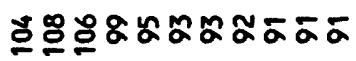

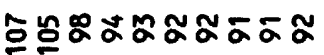

ฉッッラัタ

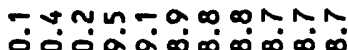

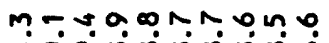

$\because-\infty \times 0$ 이 $\infty \infty \infty \infty \infty \infty$

nmom-7000.9.

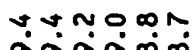

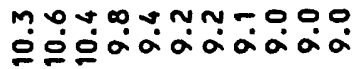

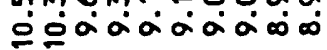
a

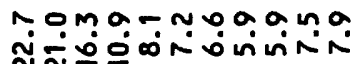

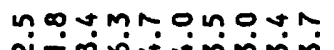
ann $\operatorname{mos}$

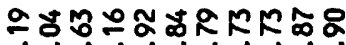
Ni--000

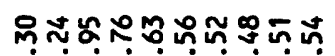
- 00000000

KR บำต 000000

00000000000

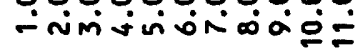

0000000000

0.00000

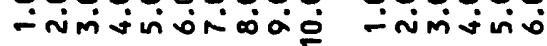

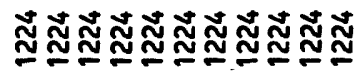

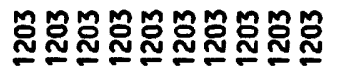

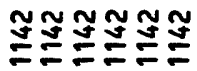

00000000000

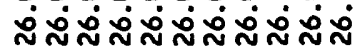

0,0000000000

0.0000

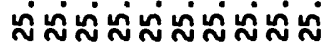

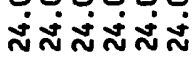




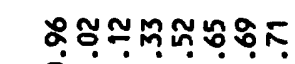

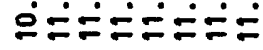

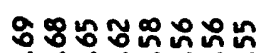

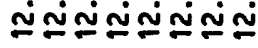

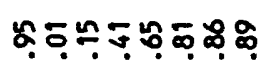

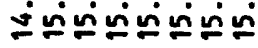

蒙

过

드으으으으프뜨

บบำำ 00000000

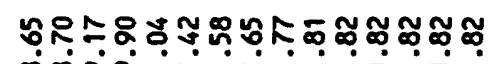

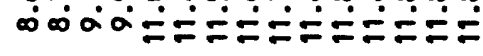

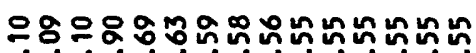

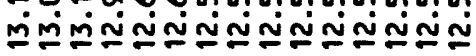

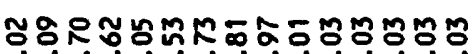

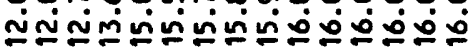

$\stackrel{\infty}{-}$

ㅇํ왜ํㅇำํํํำ

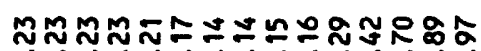

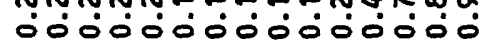

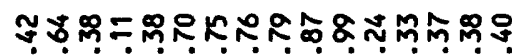

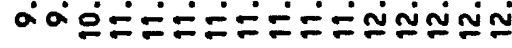

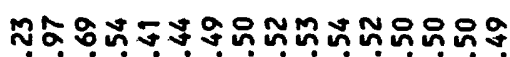

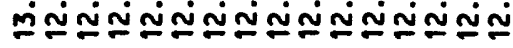

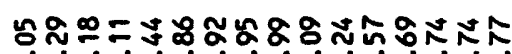

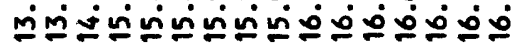

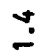

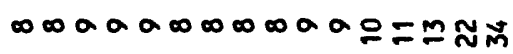

으ำ 00000000000000 $\hat{\underline{\alpha}}$

ชัญ

过

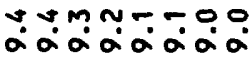

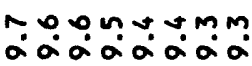

ชั

앰웡

迅志

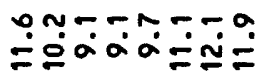

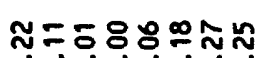

พั-

0.000000

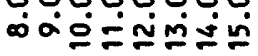

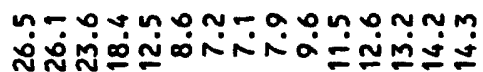

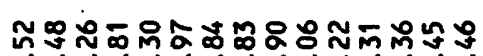

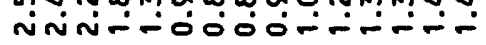

ळos.

๓̊ำ

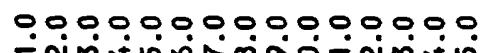

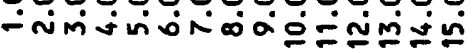

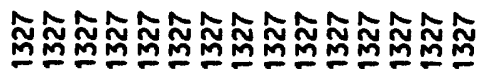

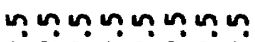

疍:

$0,0,0,0,0,0,0,0,00$

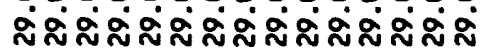

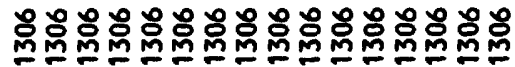

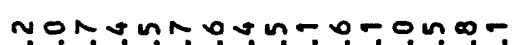

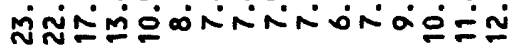

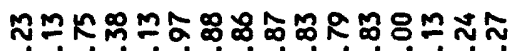

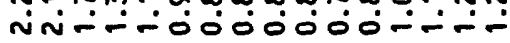

$\stackrel{\mathfrak{0}}{0}$

กิ

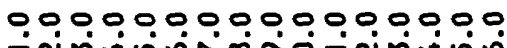

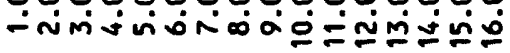

0000000000000000

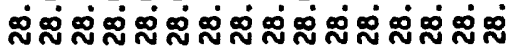




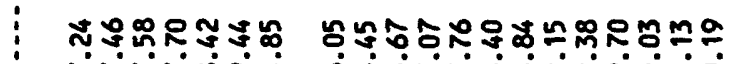

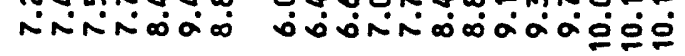

กิษสะสม

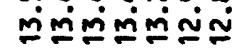

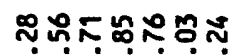

ㅇํ욤ำ

:

究

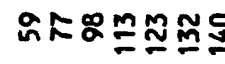

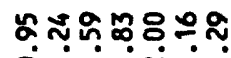

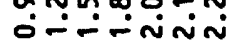

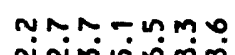

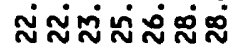
느숭ํำํำ Ninininin

崖

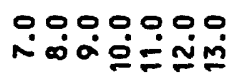

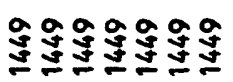

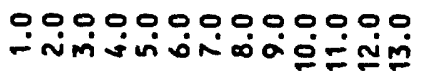

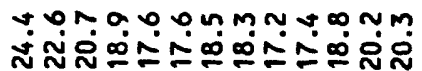

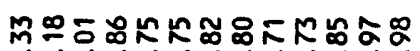

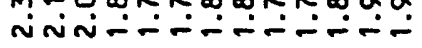

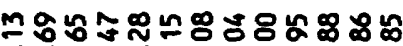

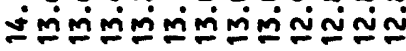

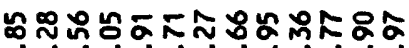

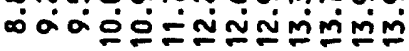

$\bar{i}$

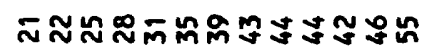

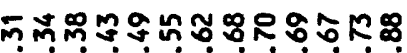
ơं000000000

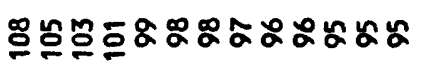

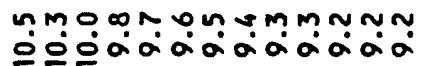

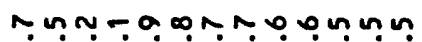

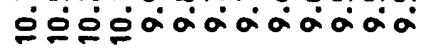

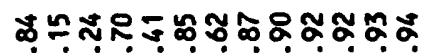

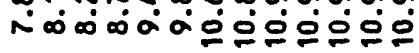

\section{\%}

$\dddot{0}$

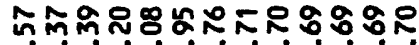

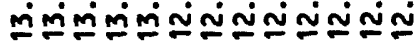

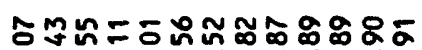

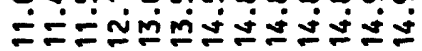

$\stackrel{\circ}{i}$

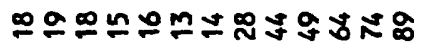

ニェニニ゚ニ

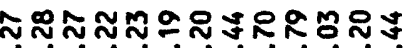

พッกำำ O0000000

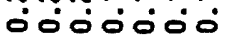<smiles>[AlH2]</smiles>

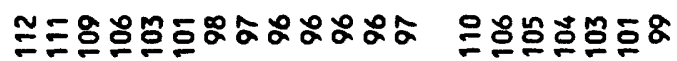

manmornmmmmmm

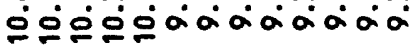

MmN-ano adannonoonono

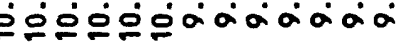

anvmnom 옹ㅇㅇㅇ

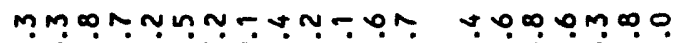

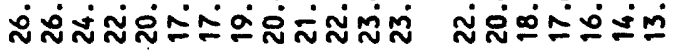

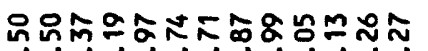

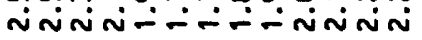

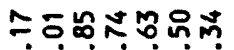
กำ-ำ

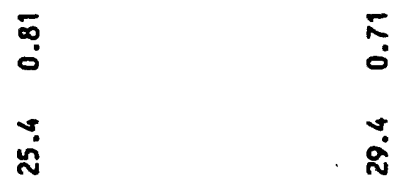

0.00000000000 0000000

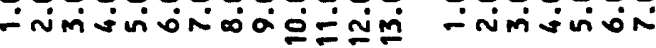

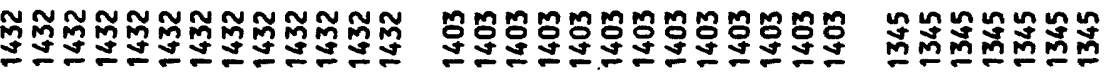




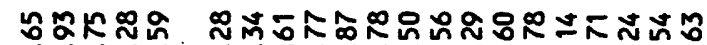

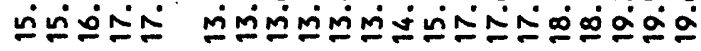

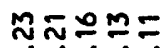

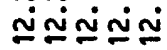

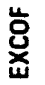

苍

客

ชัญ

褰

음

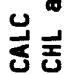

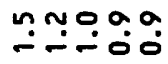

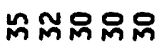
๑0ं0.

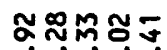

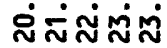

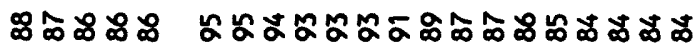

ำ

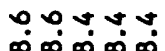

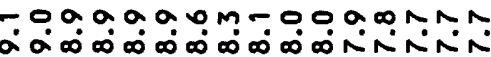

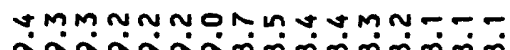

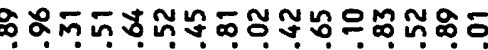

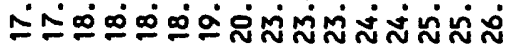

$\stackrel{9}{-}$

0000N0000Raag=

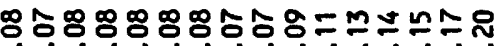
000000000000

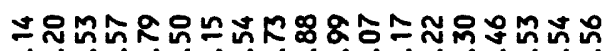

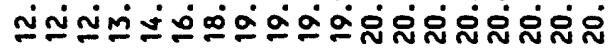

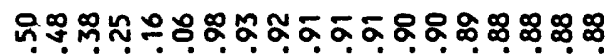

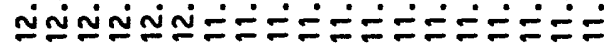

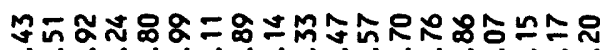

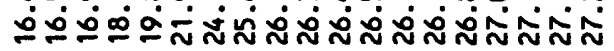

$\stackrel{9}{-}$

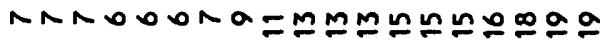

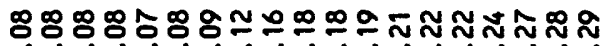
0000000000000000

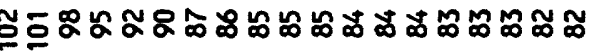

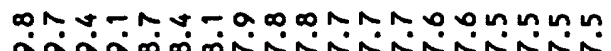
Oด.

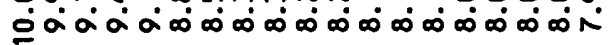

Dna- - Ommmmmmatann

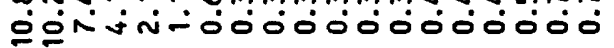

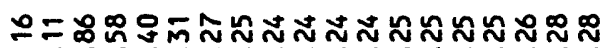
- 0000000000000000

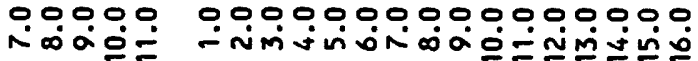

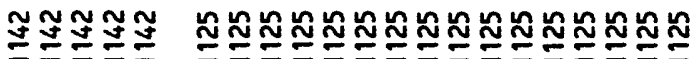

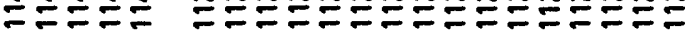

0.000

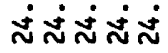

0000000000000000

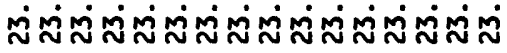

0000000000000000000

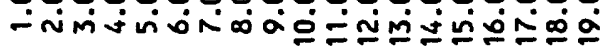

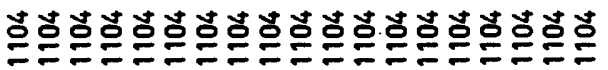

0000000000000000000

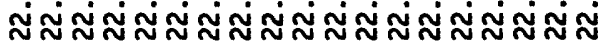




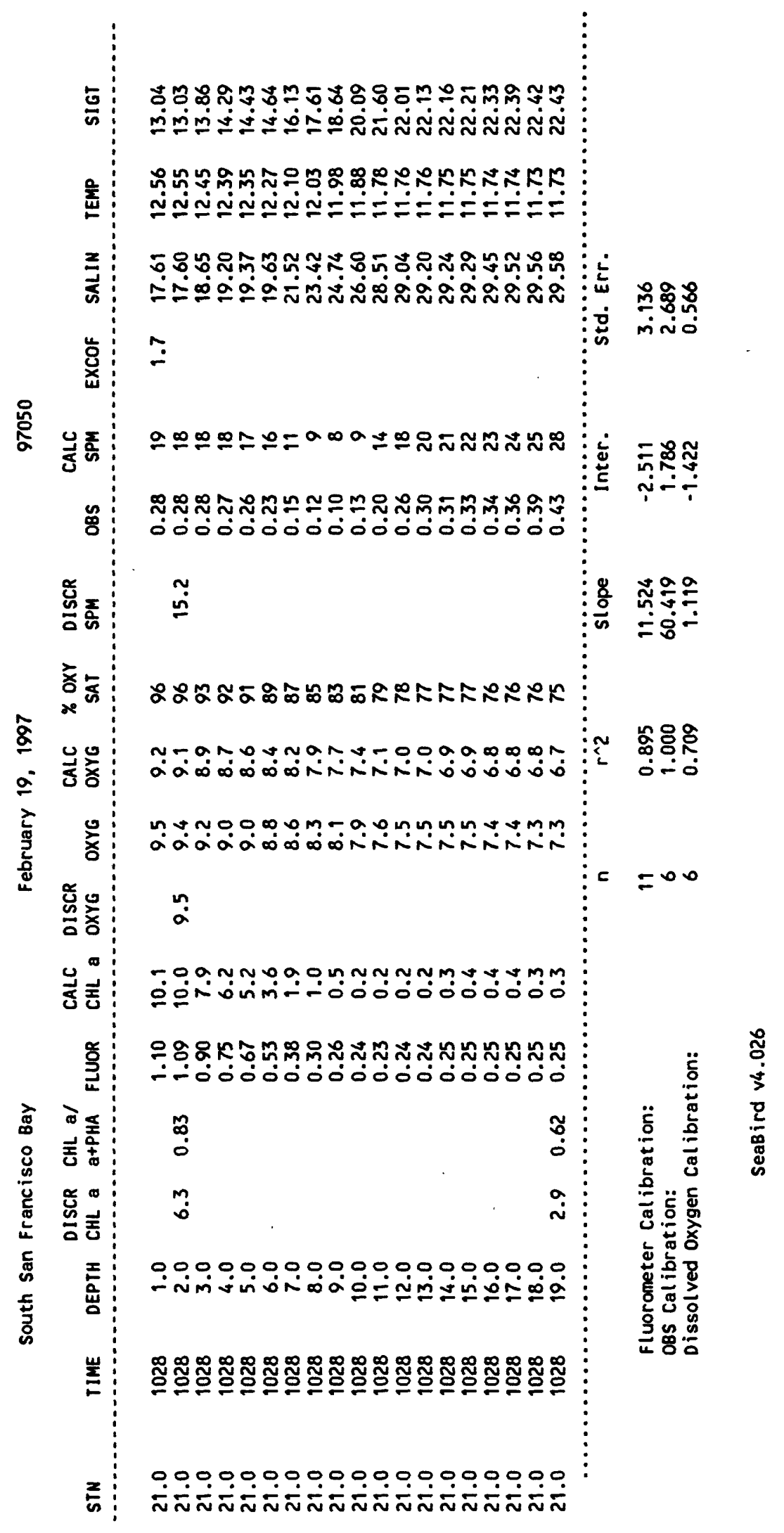


88888888888 i00000000

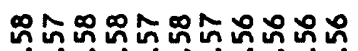

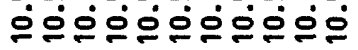

영영영ㅇㅇㅇ영 ०000000000

ஸั

容

용

各产

노ำ

옳롱

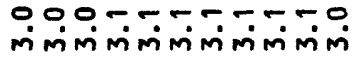

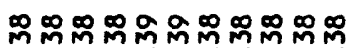
o0000000

n.

m

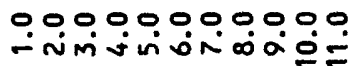

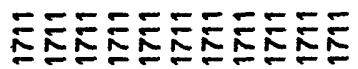

00000900000

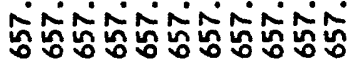

88888888888

ல00000000

NNARKRNRNNN

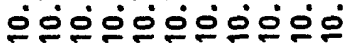

영영ㅇㅇㅇ영

0ं00000000

$\stackrel{9}{m}$

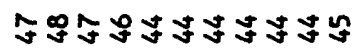

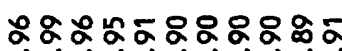
00000000000

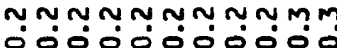
noununounou

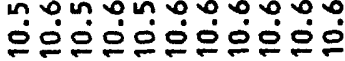

$$
\stackrel{0}{\circ}
$$

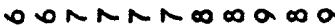
iñññññniñ

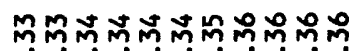
000000000

$\stackrel{?}{0}$

$\stackrel{\circ}{m}$

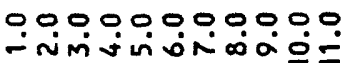

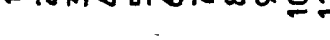

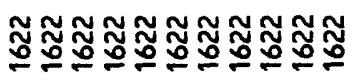

웅웅우웅우웅우웅

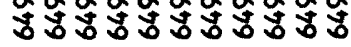

88888888888

0000000000

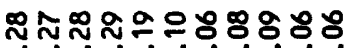

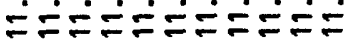

영영영영영

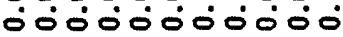

$\hat{i}$

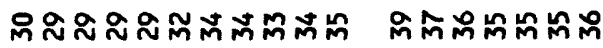

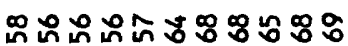

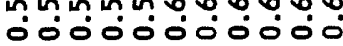

TKNRRET 0ं00000

mं

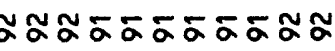

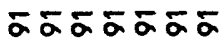

000.000005

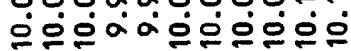

8808080

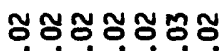
モニニモニニニ

영영영영 वं00000

$\bar{m}$

mmm nmmammus

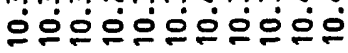

0000000 ㅇํㅇㅇㅇㅇㅇ

MMMMMmM 응ㅇㅇㅇㅇㅇㅇㅇ

\section{$\stackrel{+}{\circ}$}

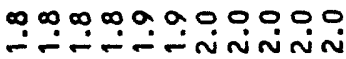

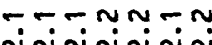
ininininis

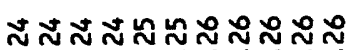
000000000

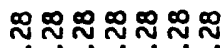
0000000

$$
\begin{aligned}
& \text { î́ } \\
& \text { i̊ }
\end{aligned}
$$

0.000000000 - Nimivionió

0.000 .00 - nimivision

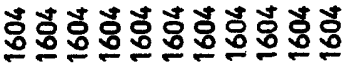

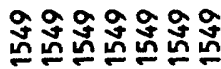

00000000000

0000000 


\section{8

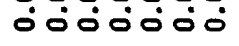

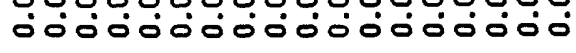

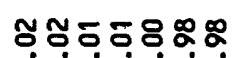 \\ ЕニニニニÓ

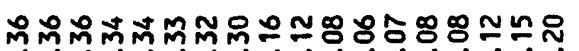

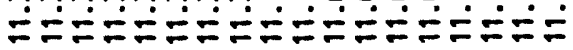

영영영

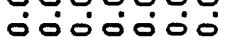

영영영영영영영영웅영영

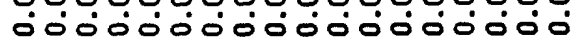

$\stackrel{\infty}{\sim}$

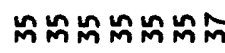

자요안

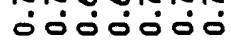

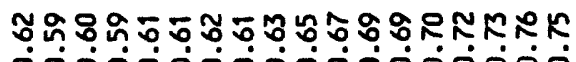

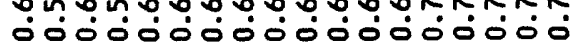

888888888888

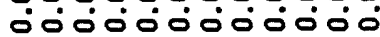

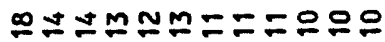

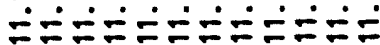

영영영영영영

ऽं00ं000000

$\stackrel{\infty}{\sim}$

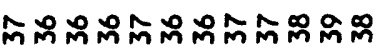

KNMNKNNลNRTR OOO000000000
888

$\dot{0} 0$

요요

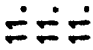

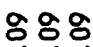

$\circ 0^{\circ}$

$\stackrel{+}{m}$

ติกำก

$5 \pi \frac{0}{5}$ ऽं०

$\ddot{m}$

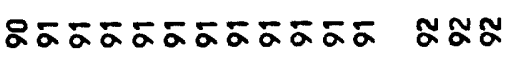

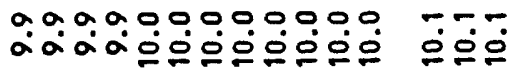

กบกกMMMmMmMM

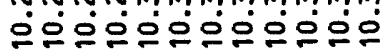

눙ํㅇㅁㅇ

$\dot{\circ}$

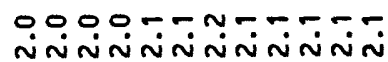

iุiํำ

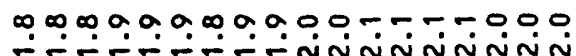

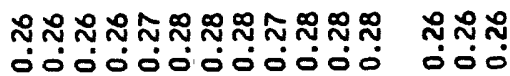

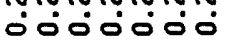

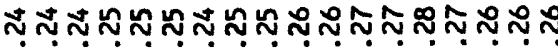

00000000000000000

นิำ

$\stackrel{\circ}{i}$

웅롱

0000000000000000000000000

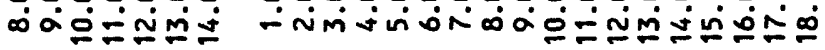

0.00000000000

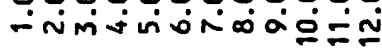

i. im

m.

$\dot{0}$

$\stackrel{+}{2}$

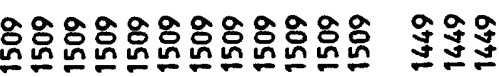

000000000000

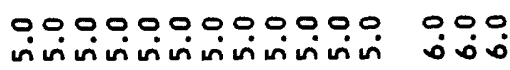


88888888 0000000

ㄷํํํํํํํํㄴ

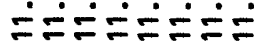

88908080

0000000

䒝

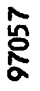

잉

过

啚

b5N5T5N5s OOOOOOOOOO
888888888888888

ó0000000000

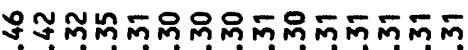

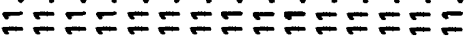

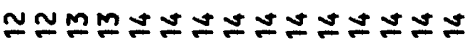
0000000000000

$\dddot{n}$

$\stackrel{\infty}{m}$

ษ

00000-ñnimivivio

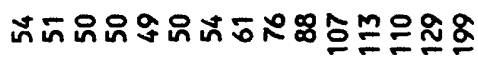

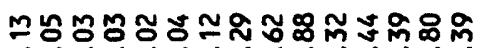

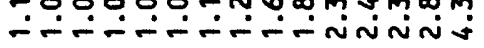

mo

00

ชัฒ

$==$

=ํํํ

$\hat{j}$

in

กับ

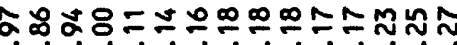

ல்0ல்

$\because$

i்

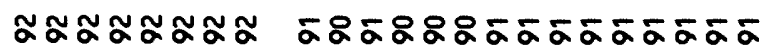

$0 \infty 0 \infty 0.090 .000 .09$

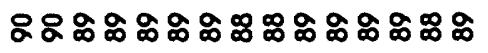

$\approx \approx$

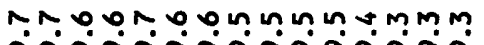

@a:

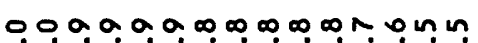

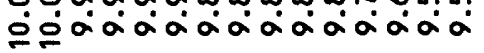

mํํำ

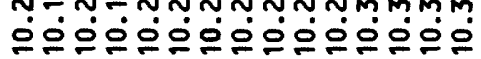

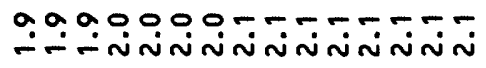

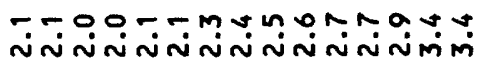

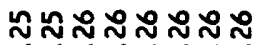
-0000000 กั

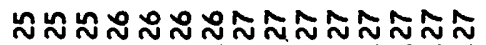
000000000000000

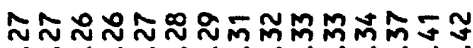
00000000000

సุ̣ ¿०

000000000000000

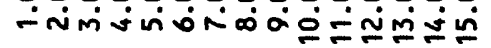

000000000000000

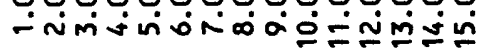
ษั $\div$ 


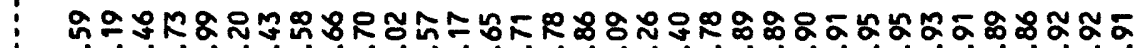

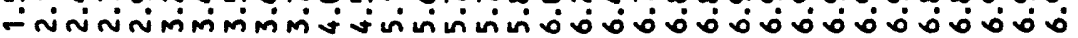

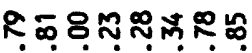

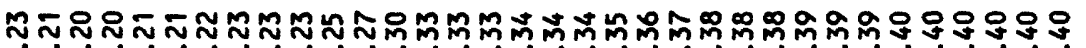

善

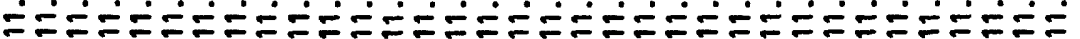

กิบก๊ก๊กักญ

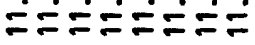

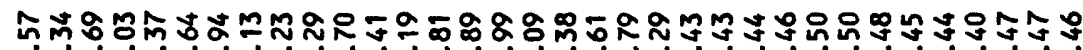

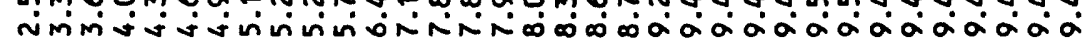

吕 -inining

\section{$\stackrel{0}{i}$}

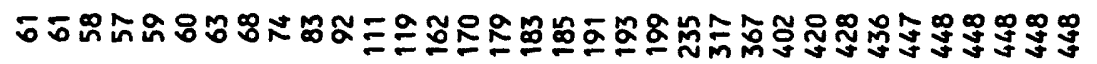

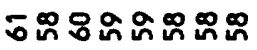

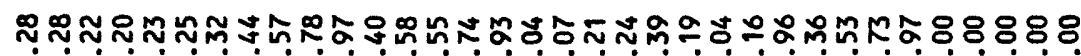

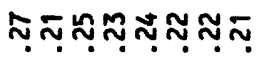

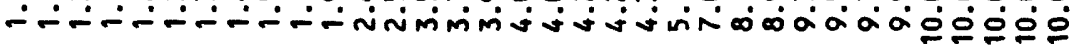

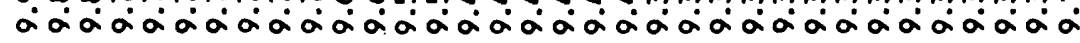

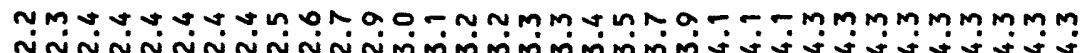

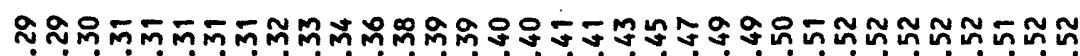
000000000000000000000000000000 战

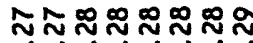

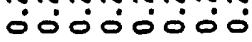

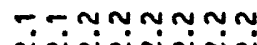
inininininin

0000000000000000000000000000000000

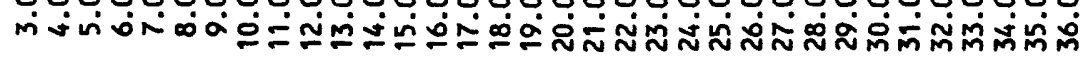

0,0000000 -nimivionió

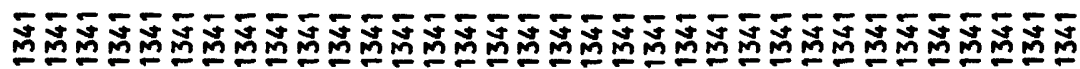

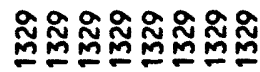

0000000000000000000000000000000000

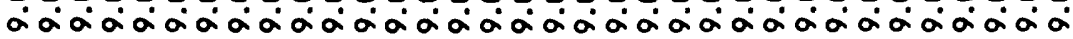

00000000 웅ㅇㅇㅇㅇㅇ 


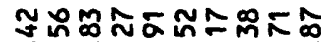
minivivinion

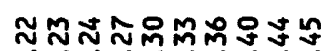
モニテニニニニニニ

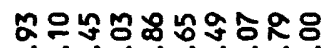
نnvivín

总

荾

웡

ชัธ์

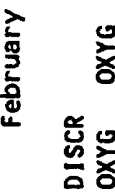

岁폰

0000000000

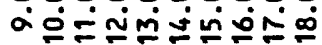

mun iñiñinimimim

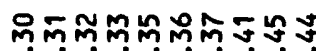

ó000000

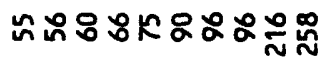

ํำำชั

$\therefore \therefore-$ niñ

ஐ̊ஐ

บง ง

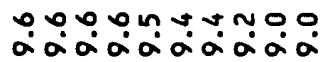

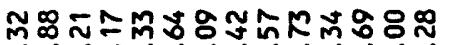

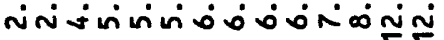

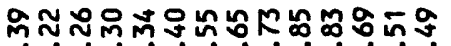

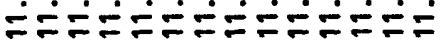

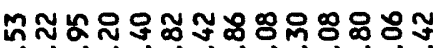

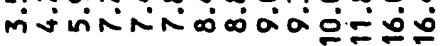

$\dot{m}$

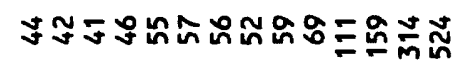

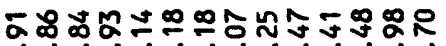

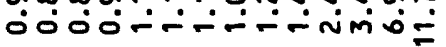

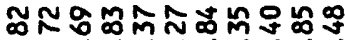

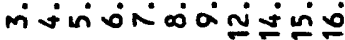

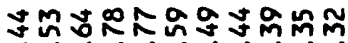
シேニテニニニニニニニ

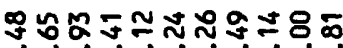

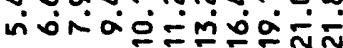

$\stackrel{n}{m}$

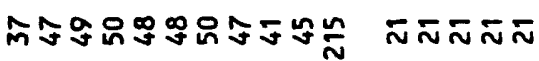

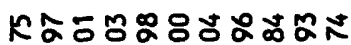

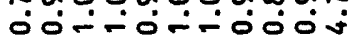

$2800 \div$

ヘヘヘ்

๓RN $\dot{\square} \dot{=}=$

ऊरำ 유웓

i

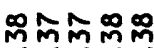
00000 $\bar{\sim}$

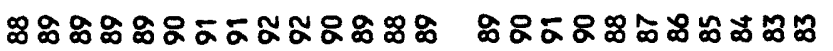

ஜタッゅロ

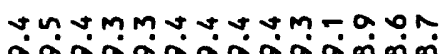

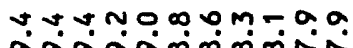

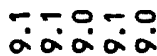

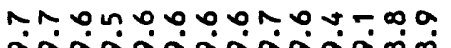

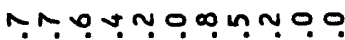

$m+m m n$ åáąa

$\bar{a}$

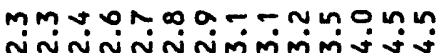

moanom $00-n$ mivmmiñiññmim

un $\operatorname{non}$ iñinin

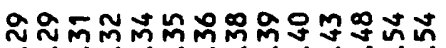

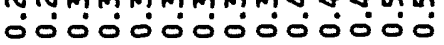

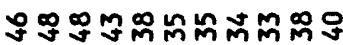
¿000000000

พิำตำำ ๑0ல0ல

$\stackrel{9}{0}$ $\stackrel{\circ}{i}$

0.0000000000000

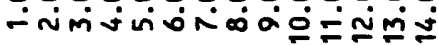

00000000000

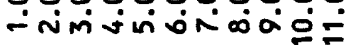

00000 - inivivin

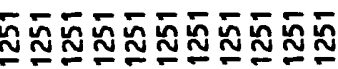

ลำลำำ

\section{0}

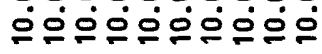

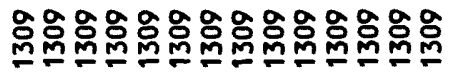

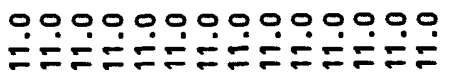




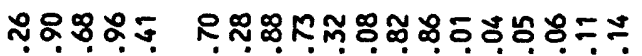

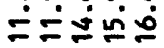

กัก๊ตัต

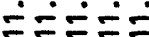

$=\Re \bar{n} \pm K$ ทำลㅊำ

产

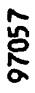

ț屯s

\section{$\bar{\sim} \bar{N} \bar{n} \mathfrak{v}$}

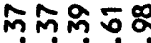

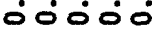

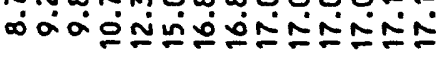

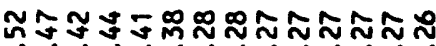

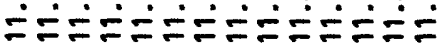

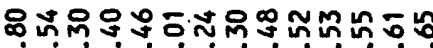

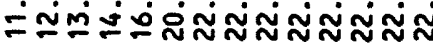

$\dddot{n}$

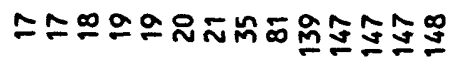

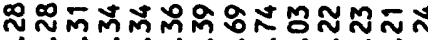

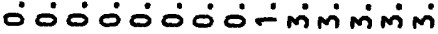

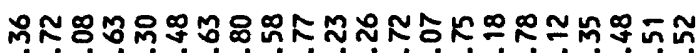

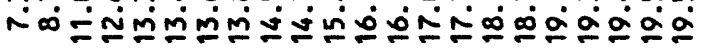

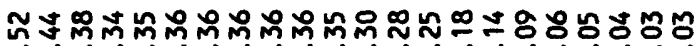

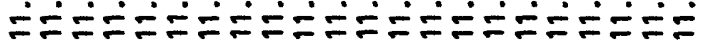

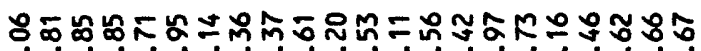

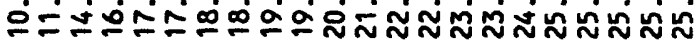

$\stackrel{0}{-}$

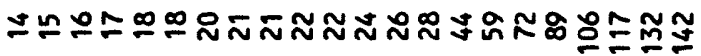

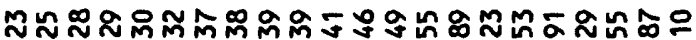

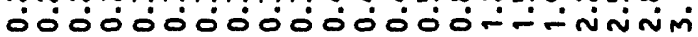

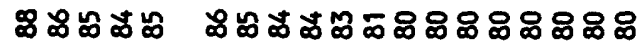

ก $\ln n-r$ $\infty \infty \infty \infty \dot{\infty} \infty$

:

:

엉

뭉뭉

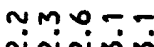
Ninmm

오ำำ

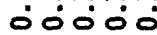

กั่

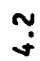

00000 லก⿻

NNNNNN

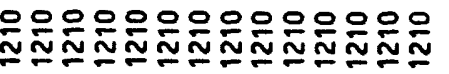

00000 minmmin
0.000000000000

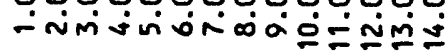

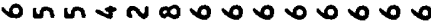

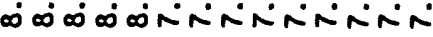

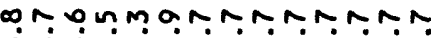
on

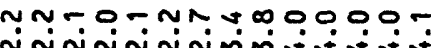

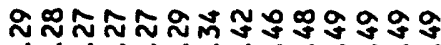

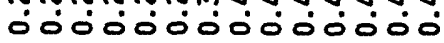

0.090000000000 ivivivivivivivi

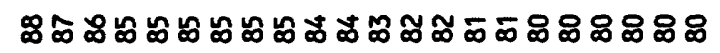

$0 \infty n+m m m m n-0, \infty \infty\{0 m n n y+4$ வ

NoRmunu tmm NoOa

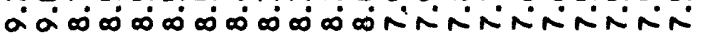

$\bar{a}$

mm

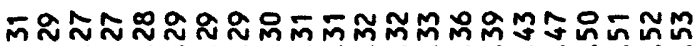

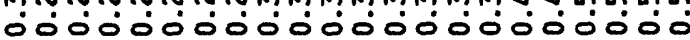

$$
\begin{aligned}
& \stackrel{n}{0} \\
& \text { i }
\end{aligned}
$$

0000000000000000000000

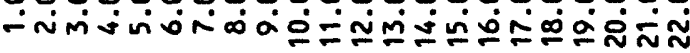

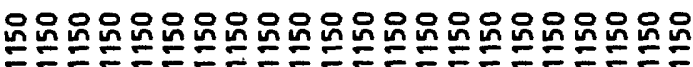

0000000000000000000000

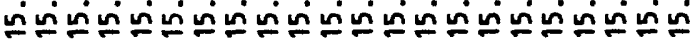


5

量:

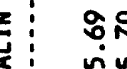

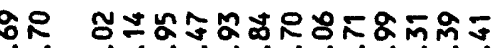

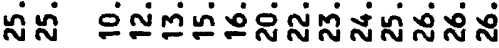

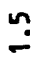

蒙

㝴紊

ธุธี

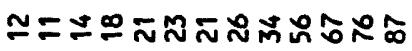

ติ जूँ

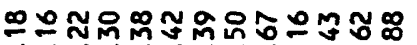

\%

000000000-

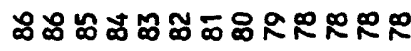

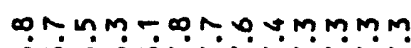

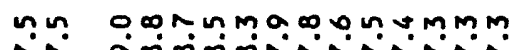

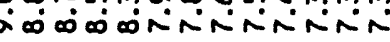

9000-5mononum

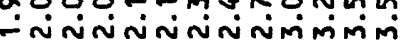

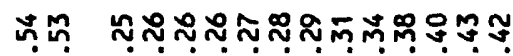

m.?.

in

000000000000 Nล -

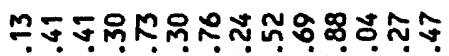

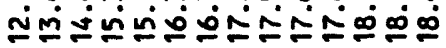

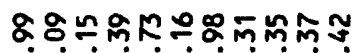
บำกำํำำำ

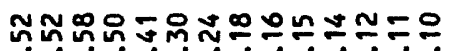

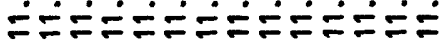

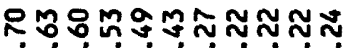

EニEニニニニニニニニ

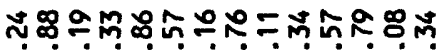

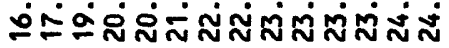

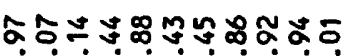

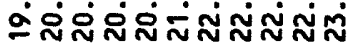
‡.

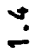

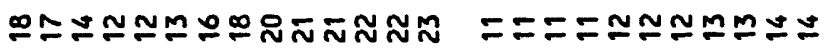

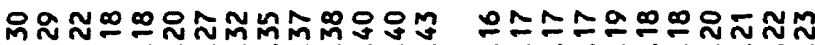

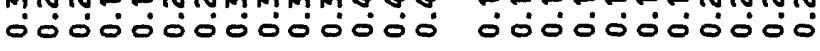

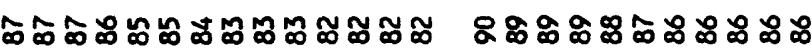

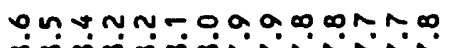

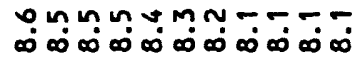

r.Mn

TrNovmmmmm क

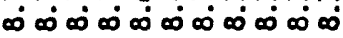
$\stackrel{\infty}{\infty}$

miññmiñ

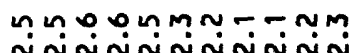

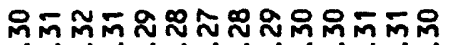
0ั000000000000

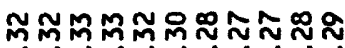

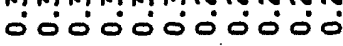

$\stackrel{\sim}{0}$ $\dot{\sim}$

00000000000000

00000000000 -

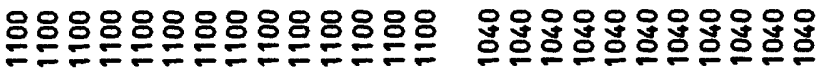

0000000000000000000000000

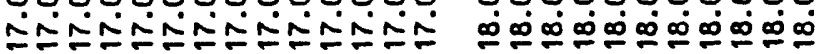




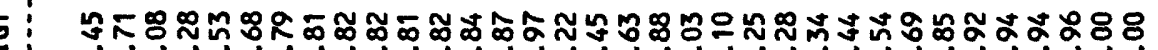

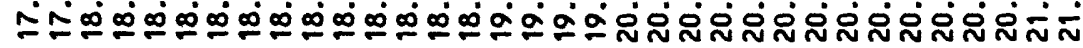

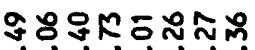

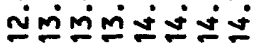

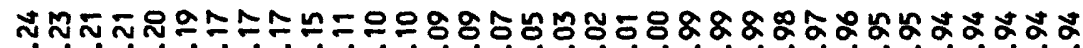

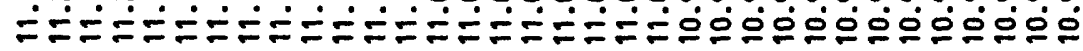

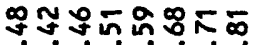

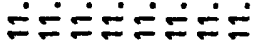

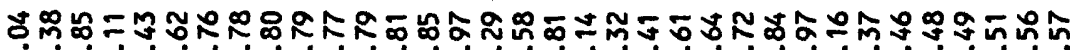

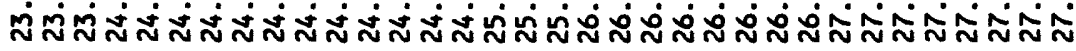

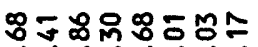

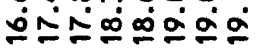

$\stackrel{0}{-}$

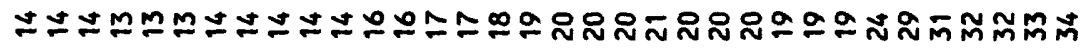

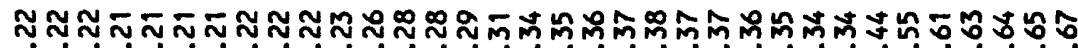

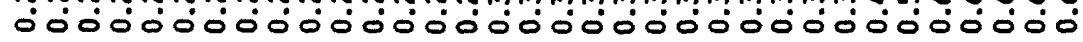

-00000000000000000NNNNNNDO0000000

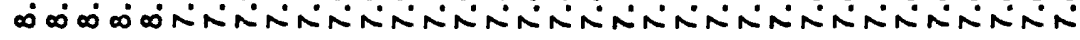

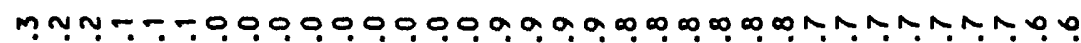
क

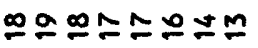

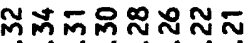

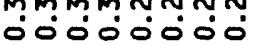

กั่

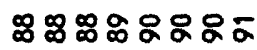

$00000 \wedge \infty$ $\infty \infty_{\infty}^{\infty} \infty \infty_{\infty}^{\infty} \infty \dot{\infty} \infty \dot{\infty}$

$\infty \infty \infty \infty \infty \infty .0$. $\infty \infty \infty \infty \infty \infty \infty \dot{\infty}$

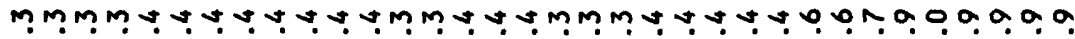

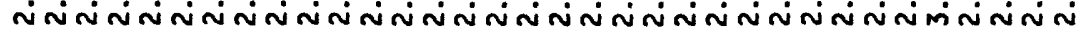

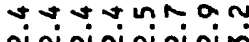

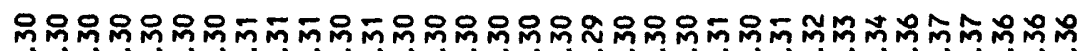
ó00000000000000000000000000000000

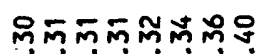
00000000

00000000 


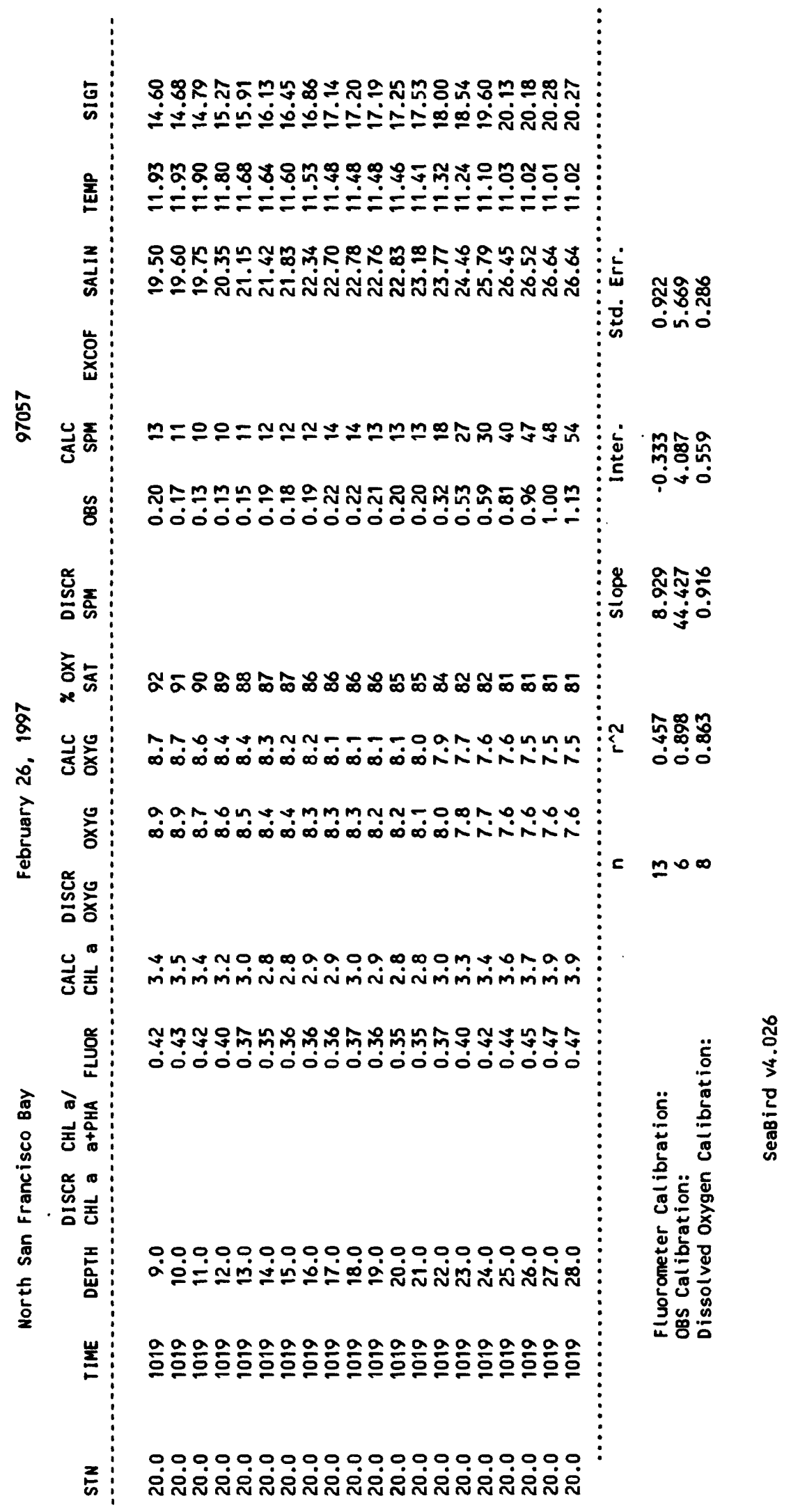




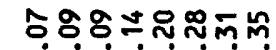 \\ ด.0.0.0.0}

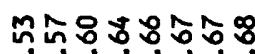

บㅗํํำำ

ㄴํำำำㅇํㅇ

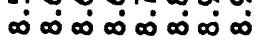

๑ะจN

NNNNR

어ำํำกำ

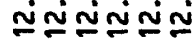

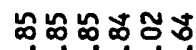

åáå

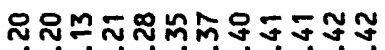

$\infty \infty_{\infty}^{\infty} \infty \infty_{\infty}^{\infty} \infty \infty_{\infty}^{\infty} \infty \infty^{0}$

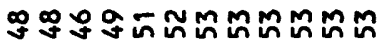

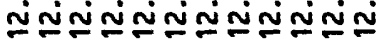

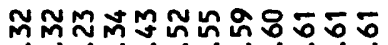

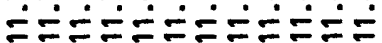

$\stackrel{+}{i}$

荢

㝴 종

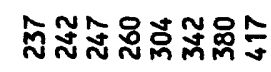

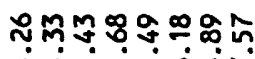

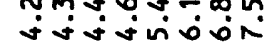

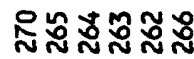

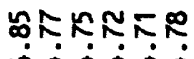

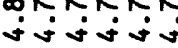

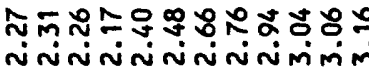

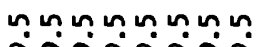

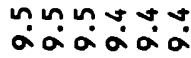

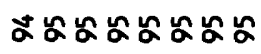

なるするณる

$\infty$

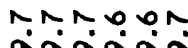

Хิ㐅े

웅

远폰

ợnomm

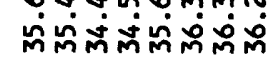

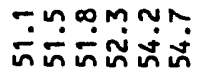

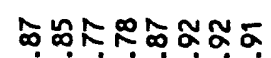
ininininin

동응ํำ

idisidu

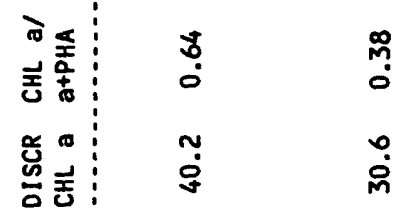

좀

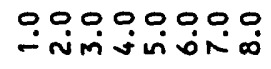

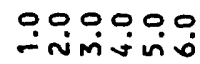

崖

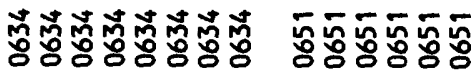

0.000000.

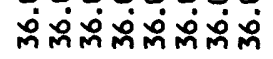

0.000 .00 लिंलेंलें

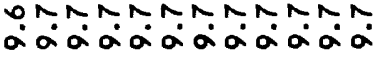

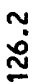

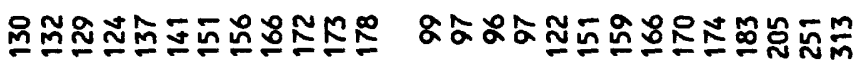

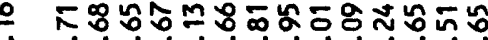

ڤั.

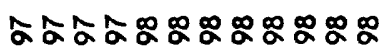

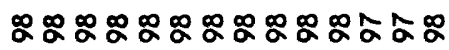

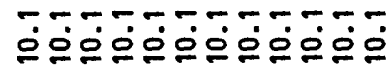

$\tilde{a}$

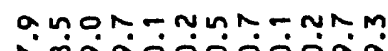

ที่

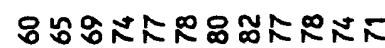

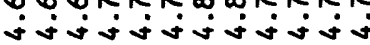

$\stackrel{\infty}{:}$

$\stackrel{8}{:}$

min

ตें

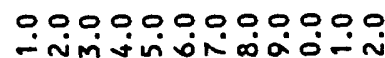

- nminuñon

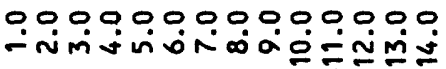

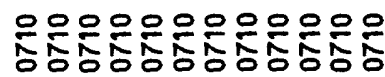

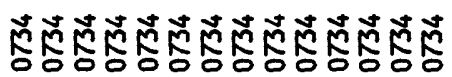

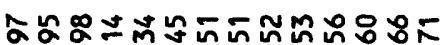

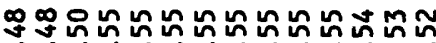

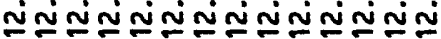

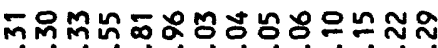

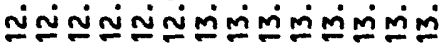

0 0 0 0 0 0 0 0 0 0nnn

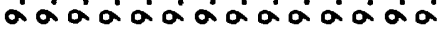

웅․ㅇ.90.9090.9.

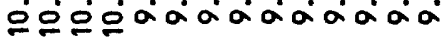

$\hat{a}$

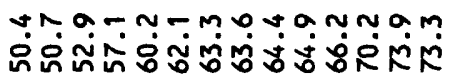

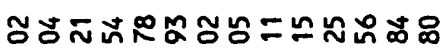

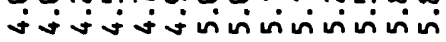

$\begin{array}{ll}\infty & 0 \\ 0 & 0\end{array}$

ğ

$\frac{5}{a}$

000000000000

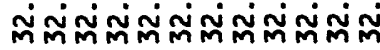

00000000000000

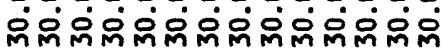




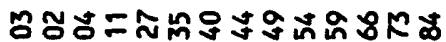

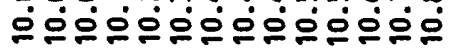

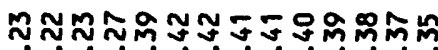

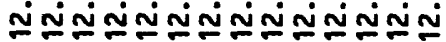

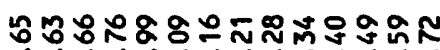

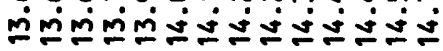

m

ถู่

ปู호

\%ั

음

ڤั)

唬

음

岇동

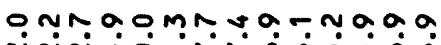

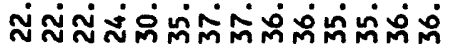

--NinimmiñNiñN

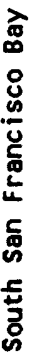

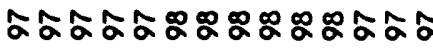

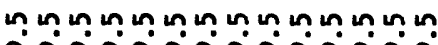

م

aаadaajaajaaa

00000000000000

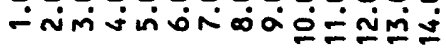

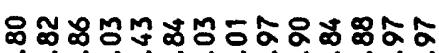

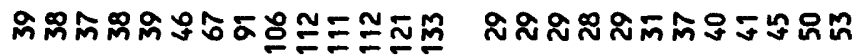

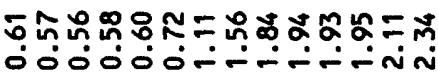

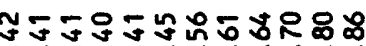

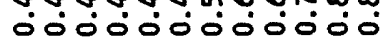

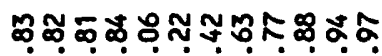

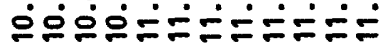

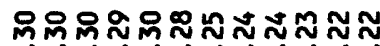
บNํำำำำ

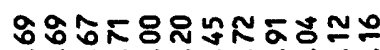

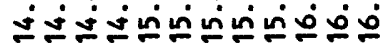

$\stackrel{n}{\sim}$

$\infty$

ஸ்

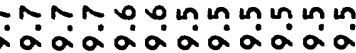

$\stackrel{\circ}{a}$

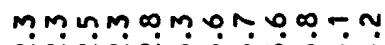

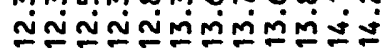

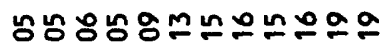

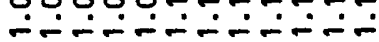

$\stackrel{0}{0}$

$\infty$

$\stackrel{\infty}{\simeq}$

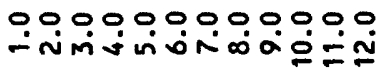

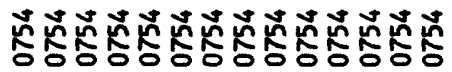

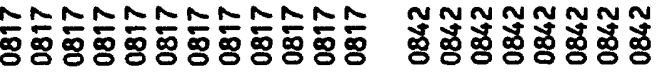

m $8582=$ 为 ํํำำmm

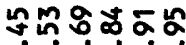
ต்றmต்றm

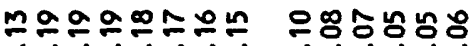
บำํำำ

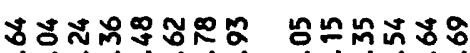

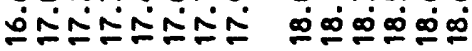
i $\quad \stackrel{m}{-}$

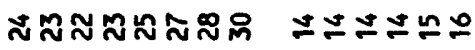

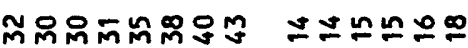

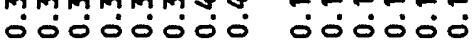

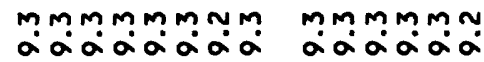

m 5 s

a

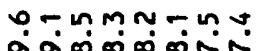

OM:NON

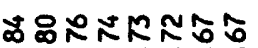

I0000000

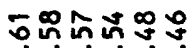

o.0.000

$\stackrel{n}{\circ}$

$\hat{0}$

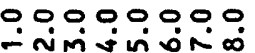

0.00000 -nmúnono -nimivio

0.09000090000000

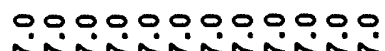

0.000000

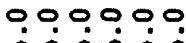

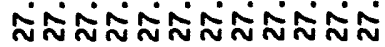

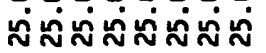

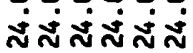




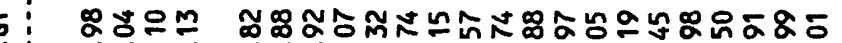

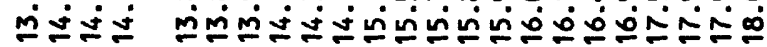

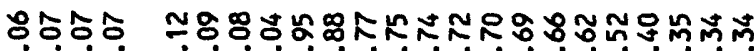
또ำ

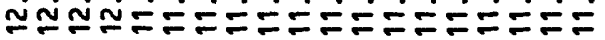

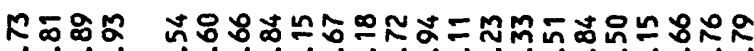

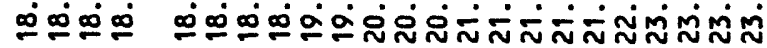
$\stackrel{\circ}{\circ}$

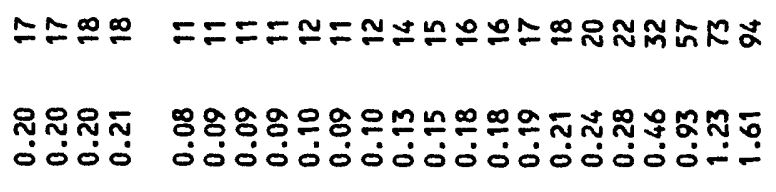

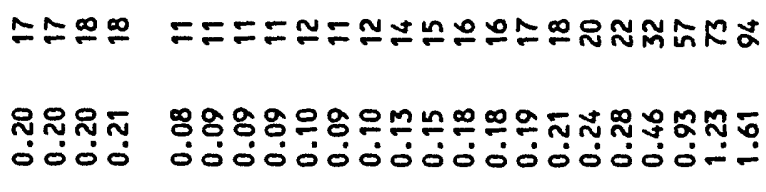

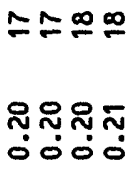

옳

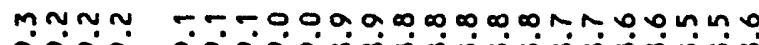

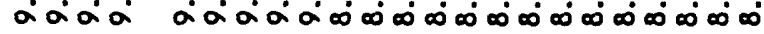

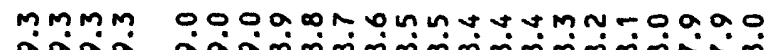

ingn

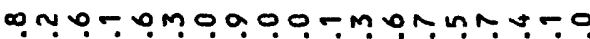
ñن

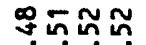
0ं0்

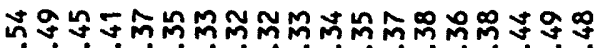
0000000000000000000

mo.

i

웅ㅇㅇㅇㅇㅇ

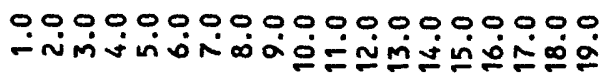

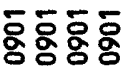

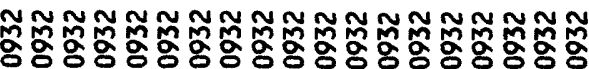

$0,0.0$ กั่ง்
0,000000000000000000

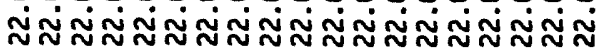

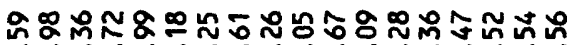

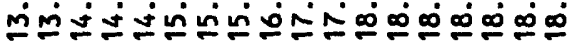

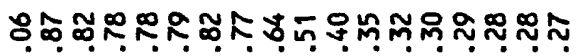

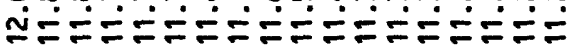

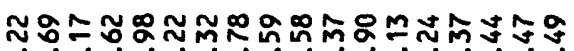

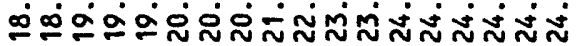
$\div$

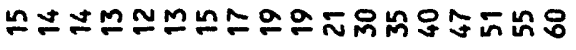

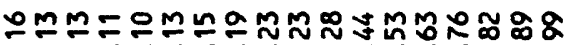
00000000000000்

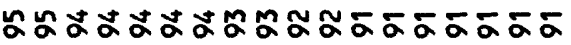

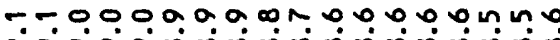

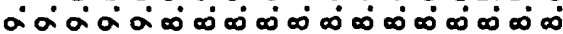

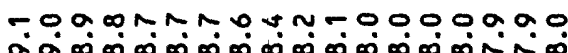
a.

ปัmoñ

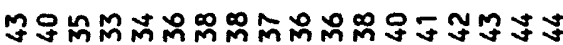

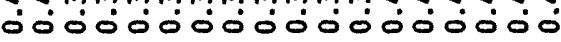

$\stackrel{0}{0}$

กี่

$\dot{v}$

$\stackrel{0}{0}$

000000000000000000

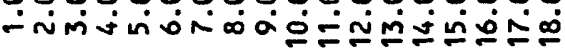

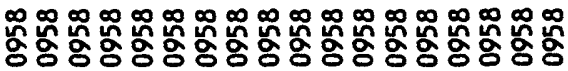

. 0.000000000000000

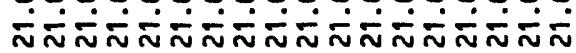




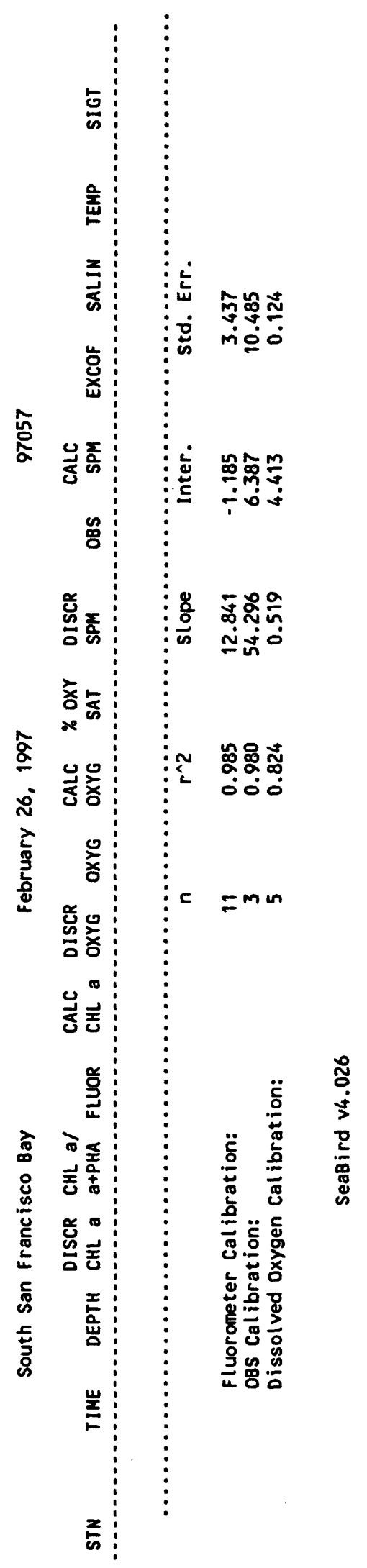




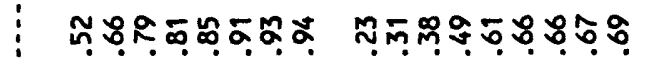

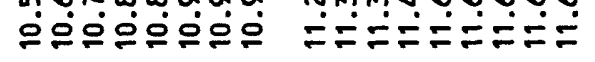

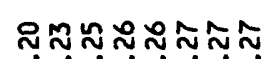

온

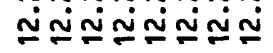

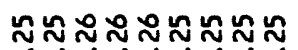
ํํํํํํํํำ

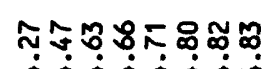

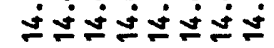
$\dot{0}$

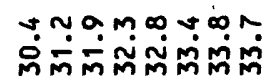

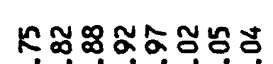
inininimimi

$$
8
$$

$\stackrel{8}{\circ}$

$\overline{\dot{m}}$

in

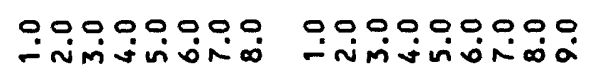

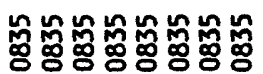

mं円mmmmm

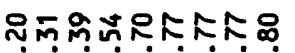

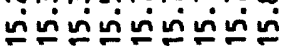

$\stackrel{\infty}{n}$

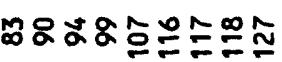

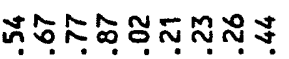
ํำ

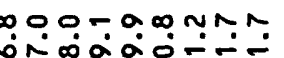

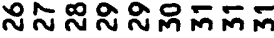

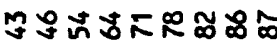
inininininin

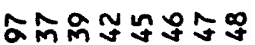

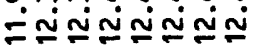

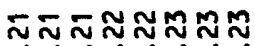
บำำํำ

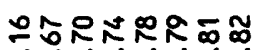
응ํㅇํำ응

$\stackrel{\infty}{;}$

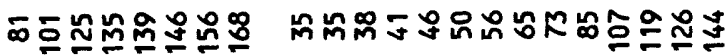

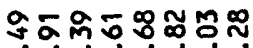
- iñiñmin

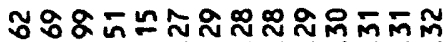

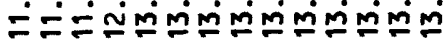

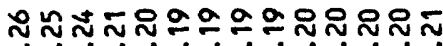

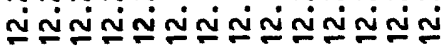

下

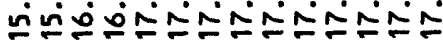

$\stackrel{a}{i}$

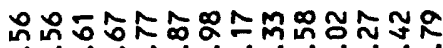

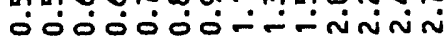

ทn ฺ-ma.0.

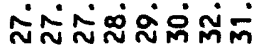

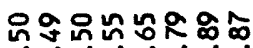
Ninininiñ mmogaop-mon Yoo

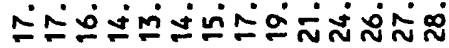

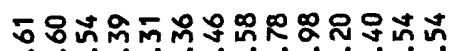
-
00000000 - imiviono

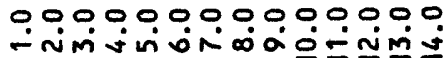




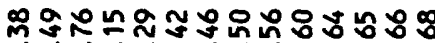

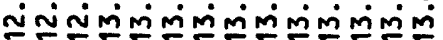

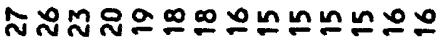

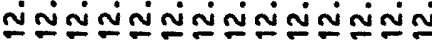

ดั

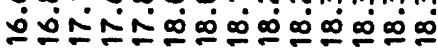
$\bar{i}$

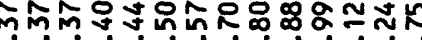
o0000000000ำ

总究:

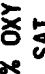

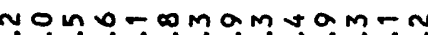

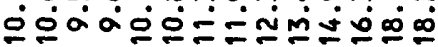

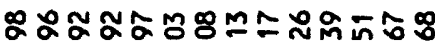

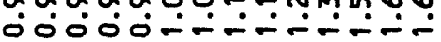

\section{8}

0

ڤิ

$\stackrel{0}{a}$

$\stackrel{a}{=}$

00000000000000 - Nimivion

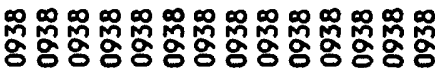

00000000000000

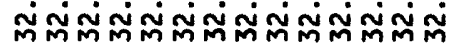

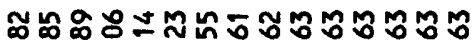

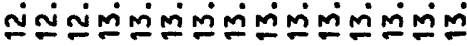

ㅊํำ능ㅇㅇ으느느는

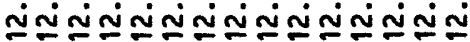

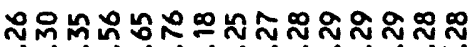

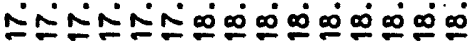
$\stackrel{\circ}{\mathrm{i}}$

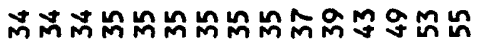

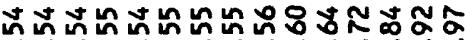
o0000000000000

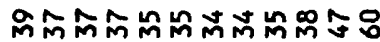
00000000000

n

N

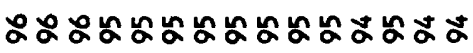

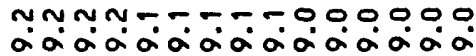

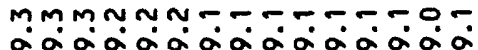

1)

mN-ONm-ammnNanm

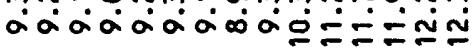

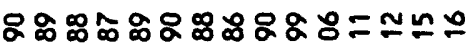

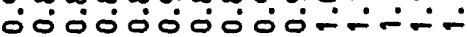

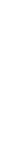

000000000000000 -

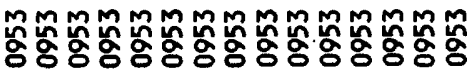

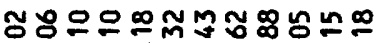

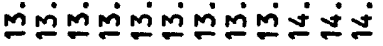

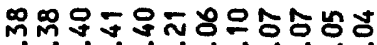

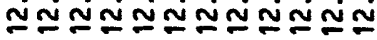

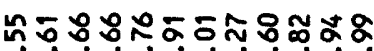

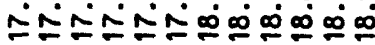

กี

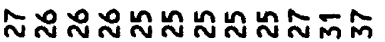

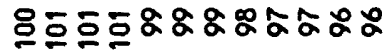

ดัơำ

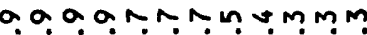

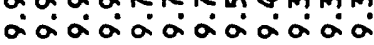

$\hat{a}$

$-\infty n-\infty \infty n m \sim \infty 00$

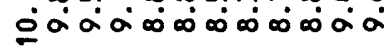

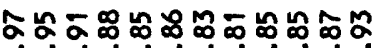
0ं000000000

?

m.

000000000000

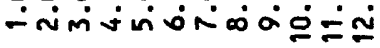

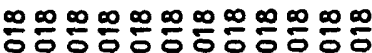

000000000000000

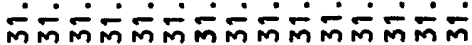

000000000000

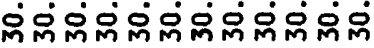


กิกี

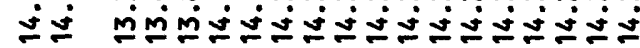

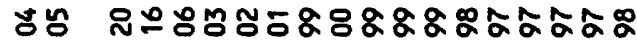

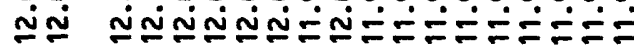

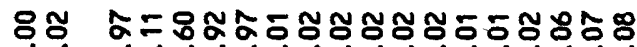

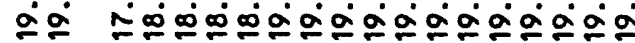

กั

๕ั

岁

요

$\stackrel{2}{\circ}$

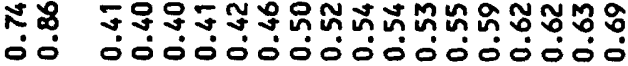

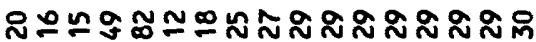

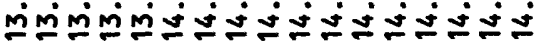

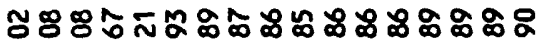

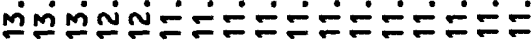

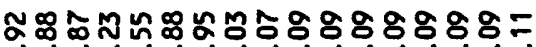

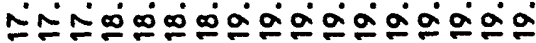

$\stackrel{m}{\text { i }}$

$\stackrel{m}{\sim}$

105: $\propto \infty \dot{0}$

กักฐ

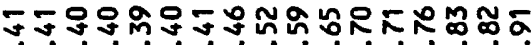

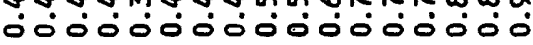

的是西要 0000

ㅇำ

M்ப̇̃

กันสั $\stackrel{\check{N}}{=}=$

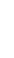

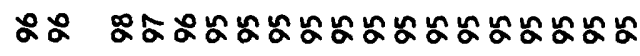

วุa

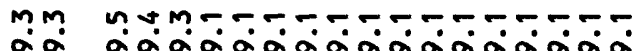
妾

옳

䢓

थே̊

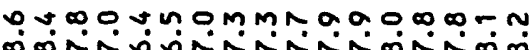

80

ఊ ó00000000000000

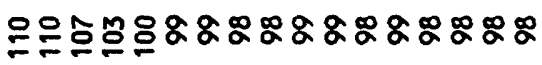

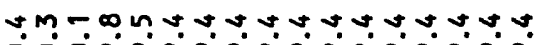

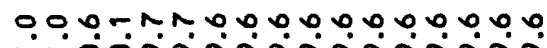

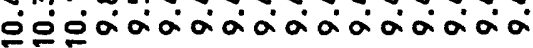

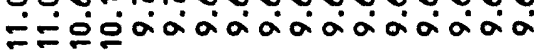

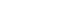

$\grave{0}$

$\stackrel{\infty}{-}$

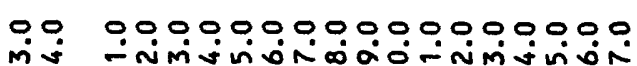

00000000000000000

0000

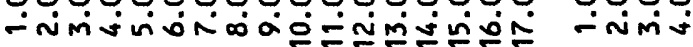

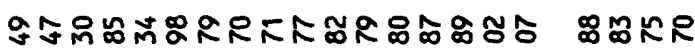

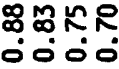




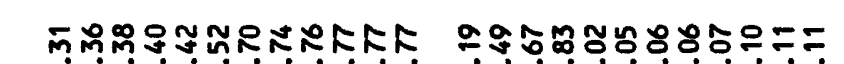

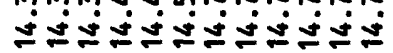

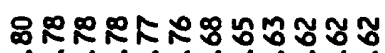

立

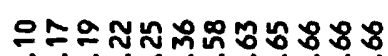

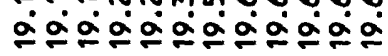

$$
\text { भूरूव }
$$$$
\text { : }
$$

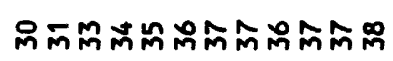

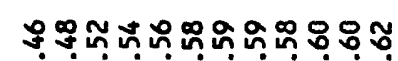

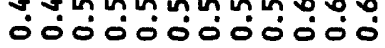

递公

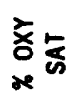

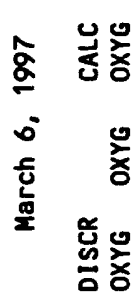

迩롱

害

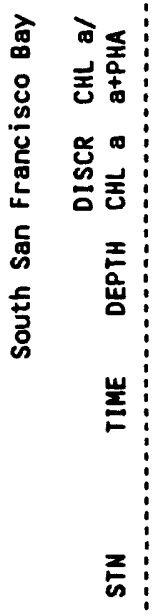

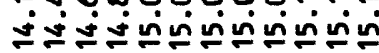

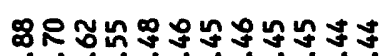

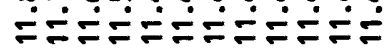

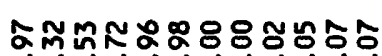

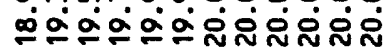

$\bar{i}$

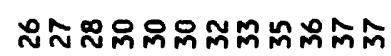

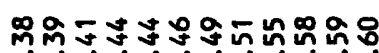

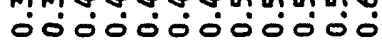

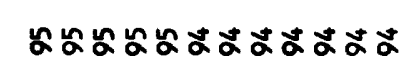

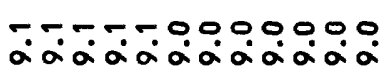

7-7-7.0000000

aiajaiajaiá

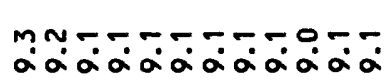

$\stackrel{\square}{a}$

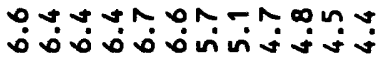

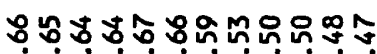

0.0000000000

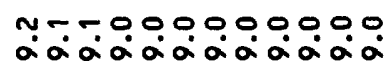

บุฒ

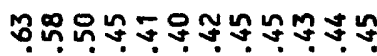

00000000000

n.

हே

$\hat{0}$

$\stackrel{\infty}{m}$

0.00000000000

ที่ن்

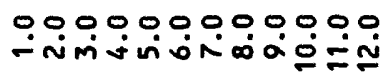

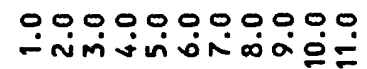

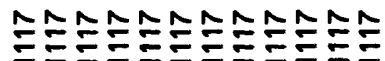

0.00000000000 ผุ

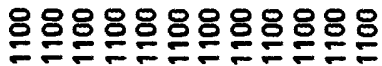

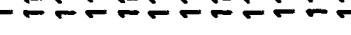

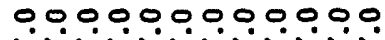

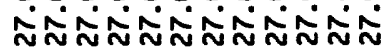

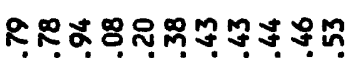

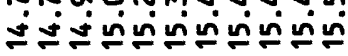

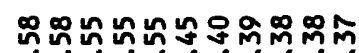

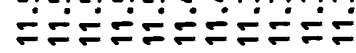

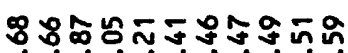

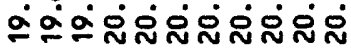

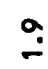

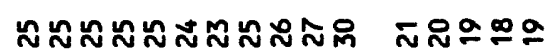

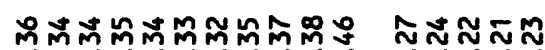
0000000000 00000

ํำำำำ

ปทำที่

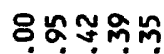

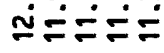

ะูษ์ะ์ ลํํํำ

$\stackrel{0}{-}$
00090

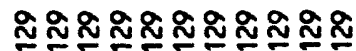

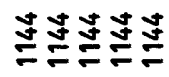

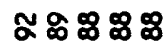

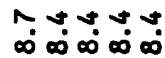

o-nNn

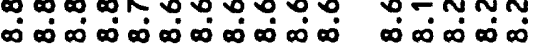

omm-ann $N \infty \infty$

no $0 \infty$

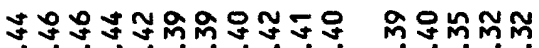
○ं000000000

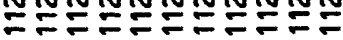

00000000000

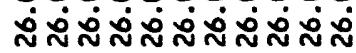

우우웅

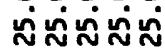




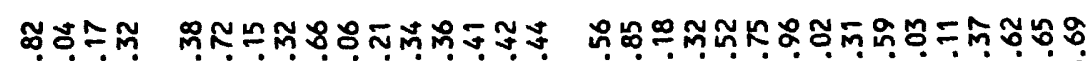

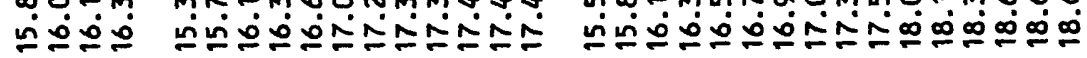

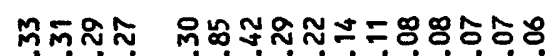

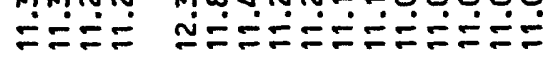

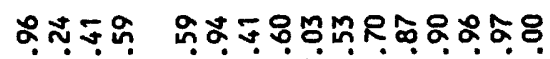

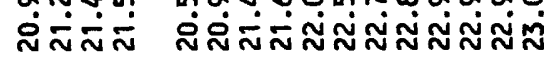

$\stackrel{m}{?}$

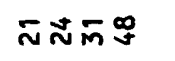

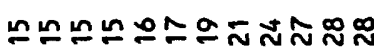

กิ่ง

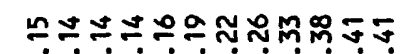
ऽo0

$\hat{m}$

䇏

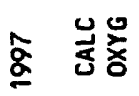

$0^{\circ}$

둔

䢔

䢔志

몽공

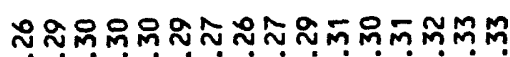

舟

Nmmininininiñ

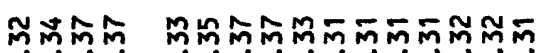

000 0000000000

$\stackrel{0}{0}$

$\stackrel{8}{:}$

$\stackrel{9}{m}$

$\stackrel{\circ}{m}$

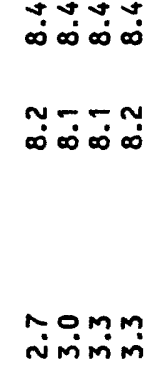

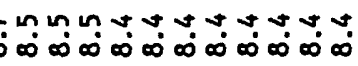

$\stackrel{\circ}{\circ}$

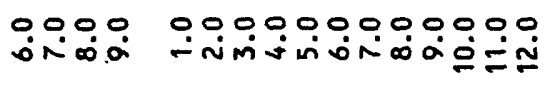

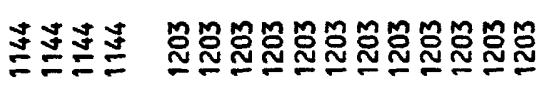

กํํํํํํ

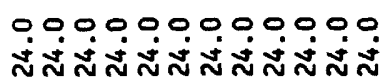

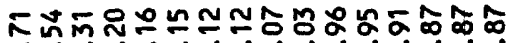

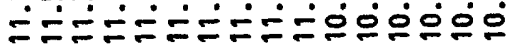

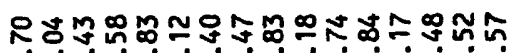

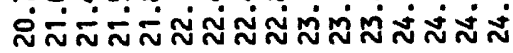

$\cong$

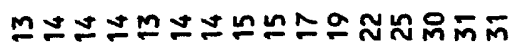

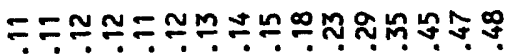

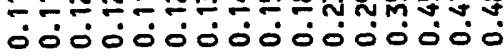

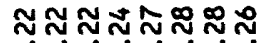

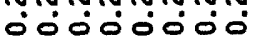

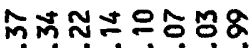
ニニニニニニニ

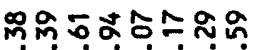

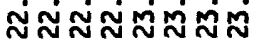

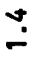

오으ำกส

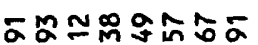
อำกำ (2)

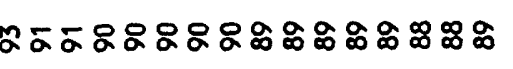

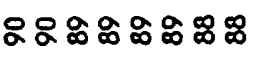

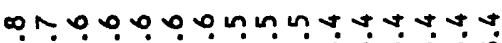

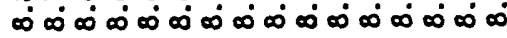

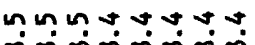

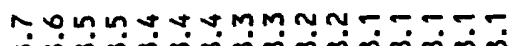

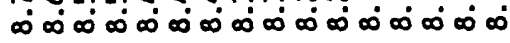

mą

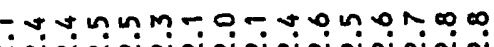

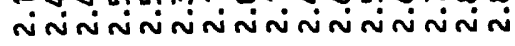

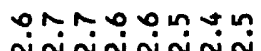
000000000000000

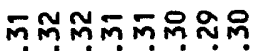
0ं000000

0000000000000000

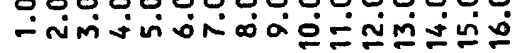

00000000 - ivivivión

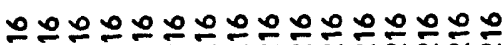

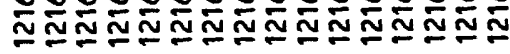

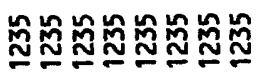

0000000000000000

0.000 .000$.

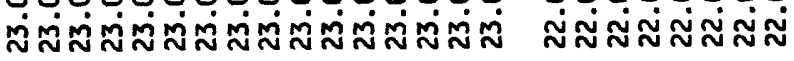




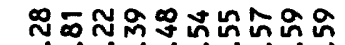
$\infty \dot{\alpha} \dot{\alpha} \dot{\alpha} \dot{\alpha} \alpha \dot{\alpha}$

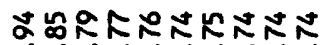
응ㅇㅇㅇㅇㅇㅇㅇㅇ

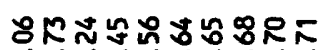

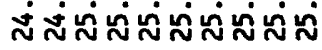

ona-mua o o o

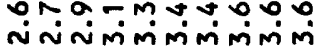

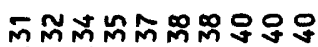
0000000000

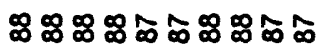

$m m m \sim N \sim N N \sim N$ $\infty \infty_{\infty}^{\infty} \infty \infty_{\infty}^{\infty} \infty \infty_{\infty}^{\infty} \infty \infty_{\infty}$

कo.909.909.9.

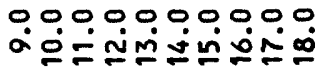

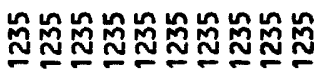

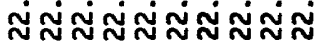

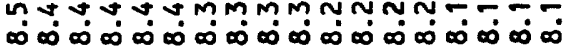
$m$

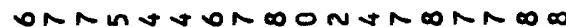

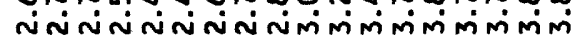

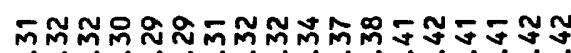

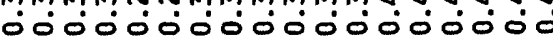
:ั ñ

000000000000000000

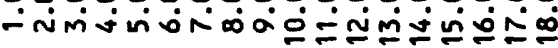

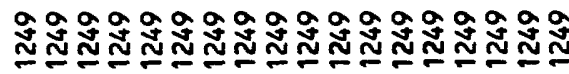

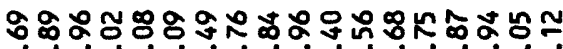

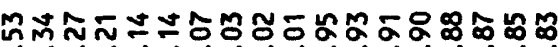

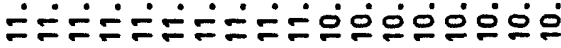

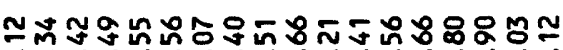

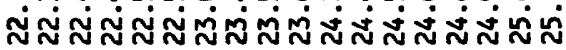
$\dddot{-}$

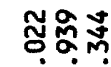
$\therefore \circ$

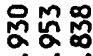
ini

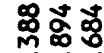

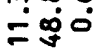

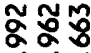
$\circ 0^{\circ}$ $\simeq 0 \mathrm{~m}$

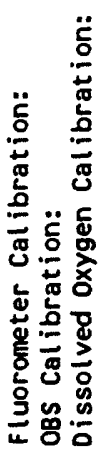

000000000000000000

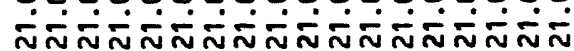




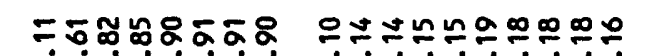

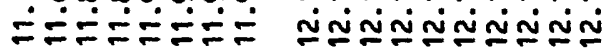

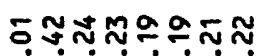

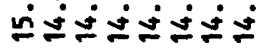

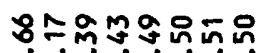
ํํㅇํํํำ

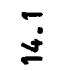

呑 造

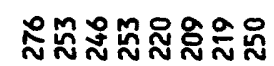

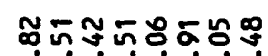

吕 minimininimín

芯

?ั:

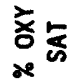

$\hat{8}$
$=$
$=$
$\frac{5}{0}$
$\frac{5}{2}$

㝴这

ํํㅇ

总

농혼

\section{monn-oom.}

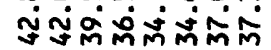

ชั iniminiminimin

ڤั

ธִ

甹旁

总

홍

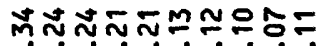

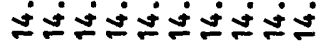

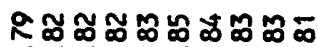

ㅇํㅇㅇํㅇํㅇํำ

$\hat{\infty}$

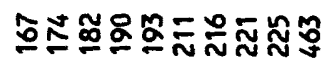

mัง

iñninimimió

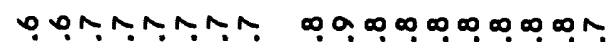

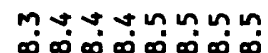
$\infty \infty \infty \infty \infty \infty \infty \infty \infty \infty)$

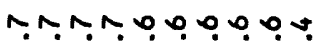

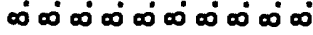

$\checkmark \infty a n \infty 0.00 \%$

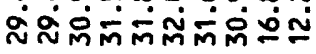

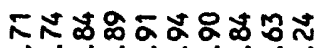

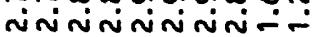

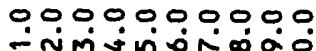

Mำำร8RKKR

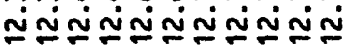

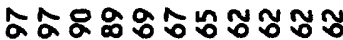

ற்ற்ற்ற்ற்ற்ற்ற

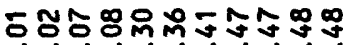

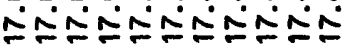

$\stackrel{n}{=}$

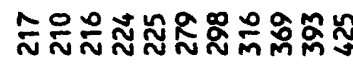

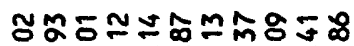
miniminimisivinin

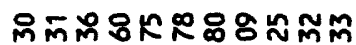

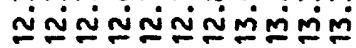

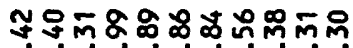

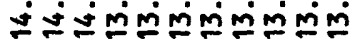

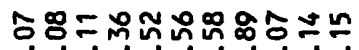

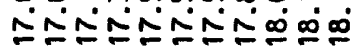

$\stackrel{n}{n}$

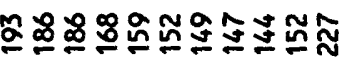

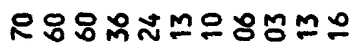
ññniñiniñm

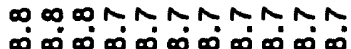

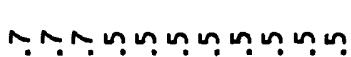
$\infty \infty \infty \dot{\infty} \infty \dot{\infty} \infty \dot{\infty} \infty \dot{\infty} \infty$
$00 \infty \infty \infty \infty \hat{N} \hat{\infty} \hat{N}$

ద $\infty \infty \omega \infty \infty \omega \infty \omega \infty \omega \infty$

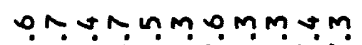

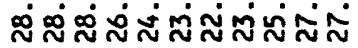

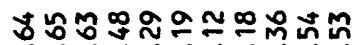
iñinininiññ

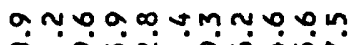

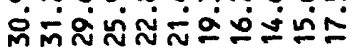

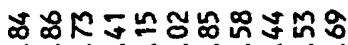
ininniñ-:-

00000000000

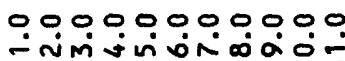

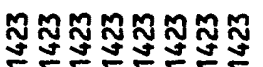

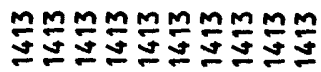

안언연연연원연연

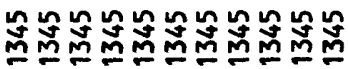

00000000

0000000000

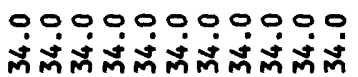

00000000000 


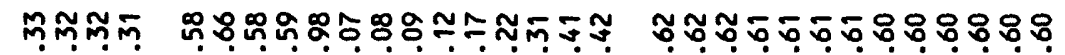

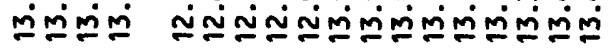

우우ํำ.

ํํํำ

$\underline{m} \div \underline{m}$

$\ddot{\infty} \infty \ddot{\infty}$

مू

总

禺

ตูลู้ส

misin

赵

ชิธู้

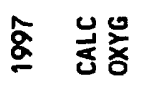

$=$

$\frac{\overline{0}}{\frac{0}{2}}$

앰

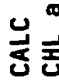

ตํํำก

$\hat{\infty} \hat{\infty} \hat{\infty} \hat{\infty}$

$\operatorname{tin} \ln _{\infty}^{\infty} n_{\infty}^{n}$

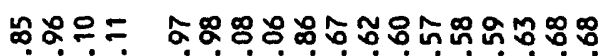

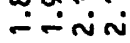

空

0.000

ํำำ

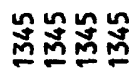

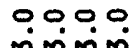

minmin

0.00000000000

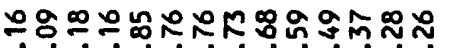

ปj寸

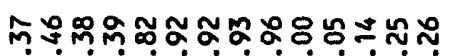

ニニ ニニニ

$\stackrel{\infty}{j}$

F⿻ำ

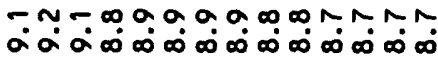

$\bar{a}$

में

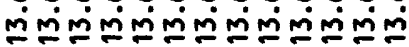

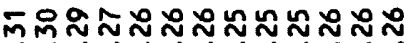

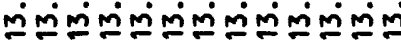

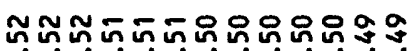

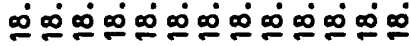

$\dot{0}$

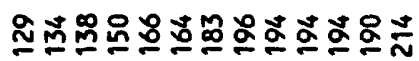

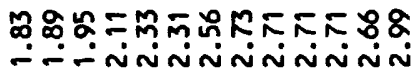

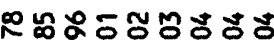

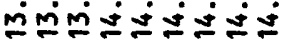

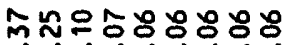

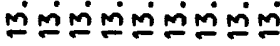

К๓ณณำกับ

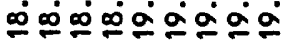

m

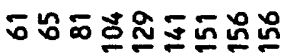

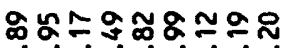
ธo- -niñ

in

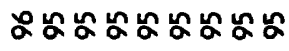

o. $\infty \infty \infty \infty, \infty, 0,0,0$

$0.0,0.0,0,00$ $\infty \infty^{\circ} \dot{\infty} \infty \dot{\infty} \dot{\infty} \infty \dot{\infty}$

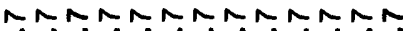
$\infty \infty_{\infty} \dot{\infty} \dot{\infty} \dot{\infty} \dot{\infty} \dot{\infty} \dot{\infty} \infty \dot{\infty} \dot{\infty} \dot{\infty} \dot{\infty}$

$\infty \wedge \wedge \wedge \wedge \wedge \wedge \sim$ $\dot{\infty} \dot{\infty} \dot{\infty} \dot{\infty} \dot{\infty} \dot{\infty} \dot{\infty}$

$$
\text { á }
$$

\%

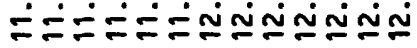

บ แ $\infty \infty \infty \circ \dot{0} \dot{\infty}=\div=$

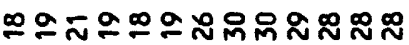
ส 2 2

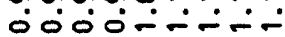

$$
\text { :̊. }
$$

乞n

$\stackrel{\circ}{\grave{i}}$

$\stackrel{+}{\check{n}}$

0.000000000000

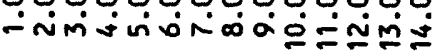

0.00000000000

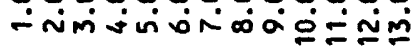

:ั

$\stackrel{\infty}{\infty}$

000000000 - imivioñó 


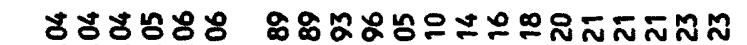

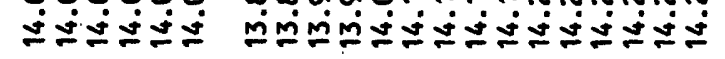

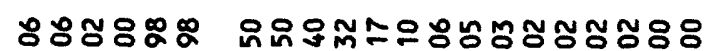

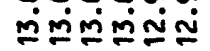

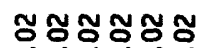

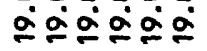

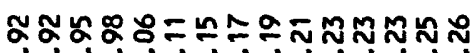

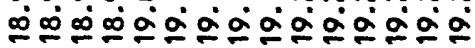

广

尽 㒮

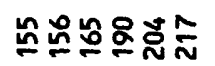

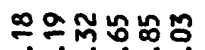
iniñinm

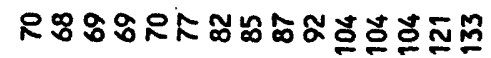

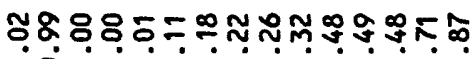

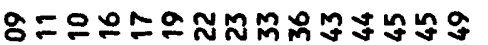

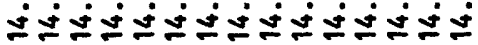

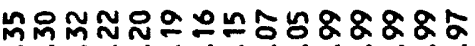

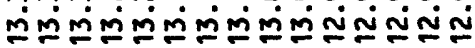

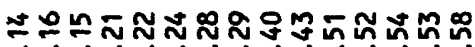

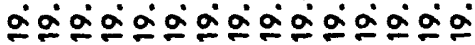

$\tilde{n}$

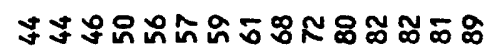

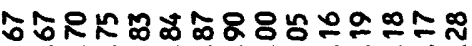

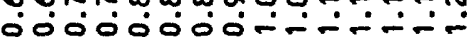

Бะณ

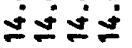

ํㅡㄴํํㅇ ற்றलि

$\mathfrak{\infty}$ gia்

m.

ฆลํำ

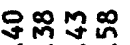
象象象

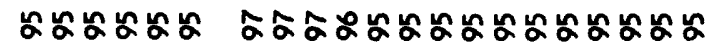

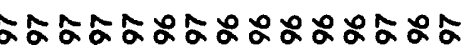

ัะะำ

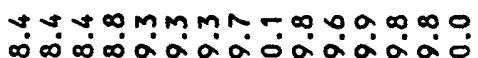

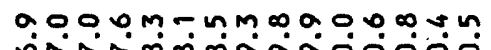

$\operatorname{con}+\infty$

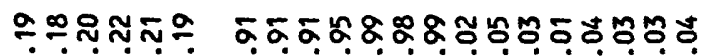
$\therefore \because \dddot{\square}$

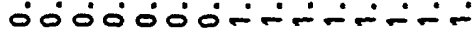

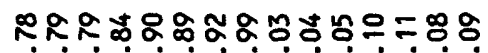
00000000

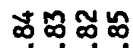

กํ.

000000000000000

0000

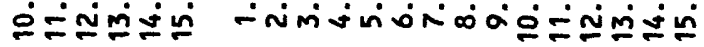

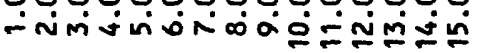
-nimis 


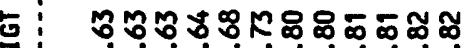

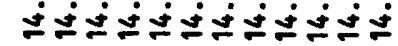

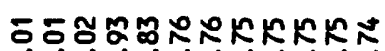

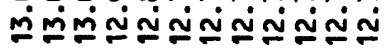

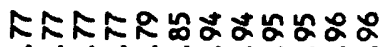

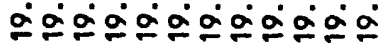

递

崖

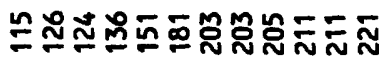

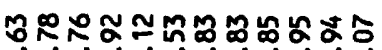
- -ñNiñNinm

$\because$

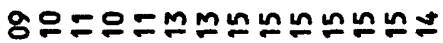

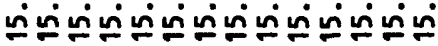

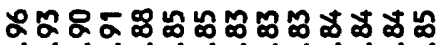

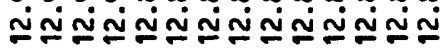

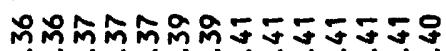

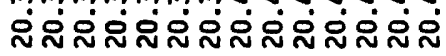

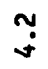

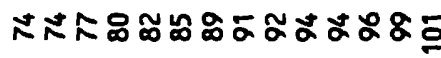

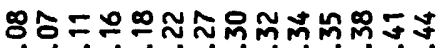

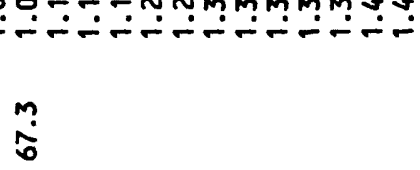

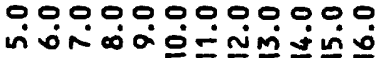

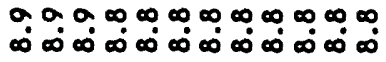

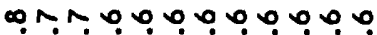

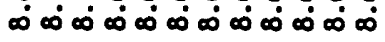

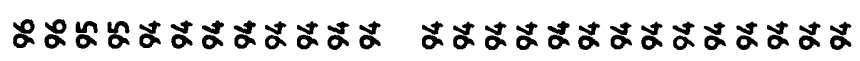

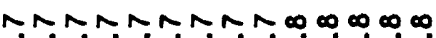

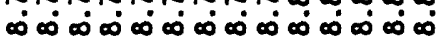

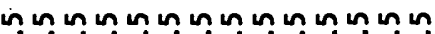
$\infty \dot{\infty} \infty \dot{\infty} \infty \dot{\infty} \infty \dot{\infty} \infty \dot{\infty} \infty \dot{\infty} \infty \dot{\infty}$

$\underset{\infty}{\infty}$

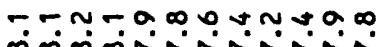

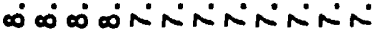

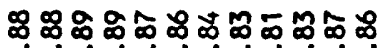
ல0ं00000000

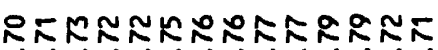
o000000000000<smiles>[CH]=[Co]</smiles>

$\overline{0}$

$\tilde{n}$

00000000000000

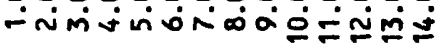

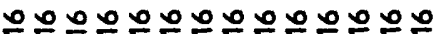

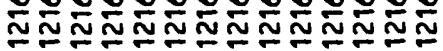
ฉి

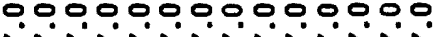

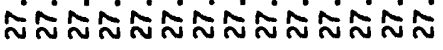

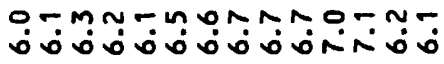

8영RRRR음

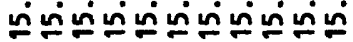

นิำ อำ

מRKKKRKKRㄴ

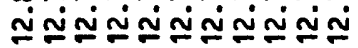

ณัธก บำ 영을ニЕ=ニ웅

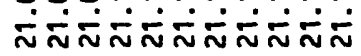

กิกั ஸ்亠े $\vec{j}$ $\stackrel{\infty}{\sim}$

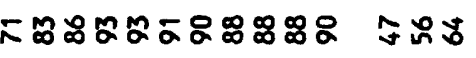

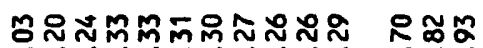

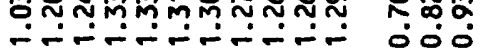

a.g.a-nN-ONUM ininimimimimimim

$\infty$

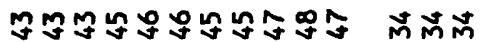

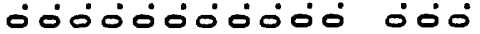

00000000000000

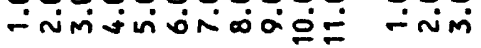

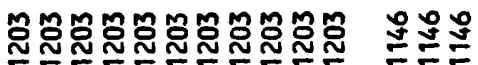

00000000000000

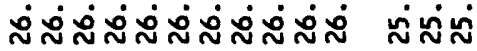




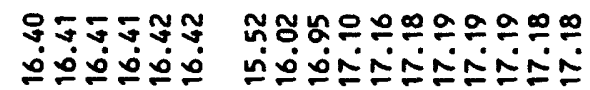

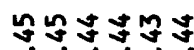

똚ำ

ณณณัณล์

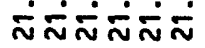

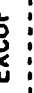

号 范

タก゚Rละ

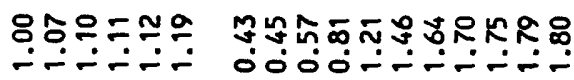

$\underset{\infty}{n}$

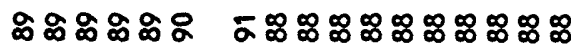

$m m m m m m$

$\infty \infty \infty \infty \infty \infty$

$\infty \infty \infty \infty \infty$

NiñNán

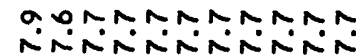

$\stackrel{m}{\infty}$

迆롱

gongogin

iñ.

ติํำำกำำ

00000

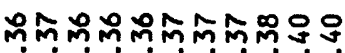

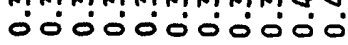

:ั:

$\stackrel{\mathbb{4}}{\stackrel{5}{0}}$

i

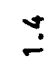

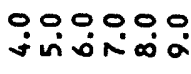

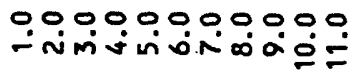

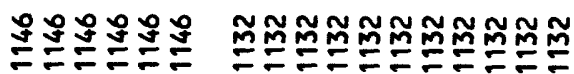

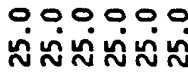

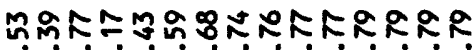

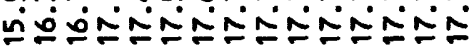

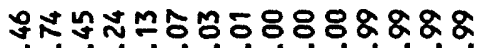

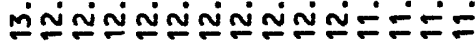

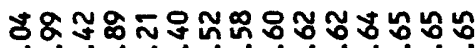

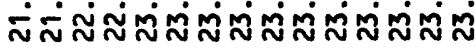

$\stackrel{\circ}{i}$

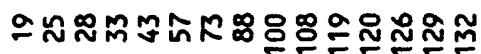

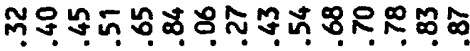

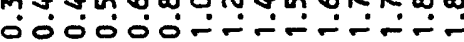

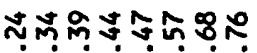

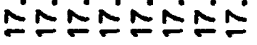

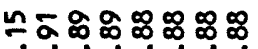

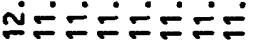

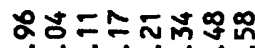

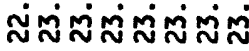

$\bar{n}$

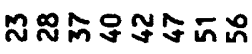

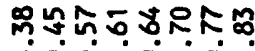
O००000000

ๆ

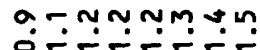

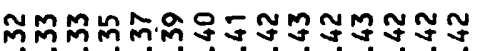

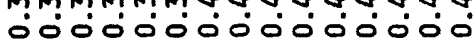

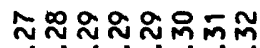
क0000000

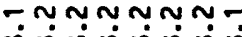

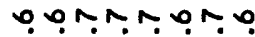

mon?

nNiñNa

000000000000000

-

00000000

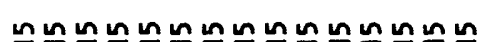

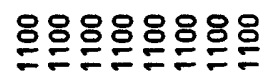

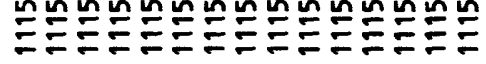




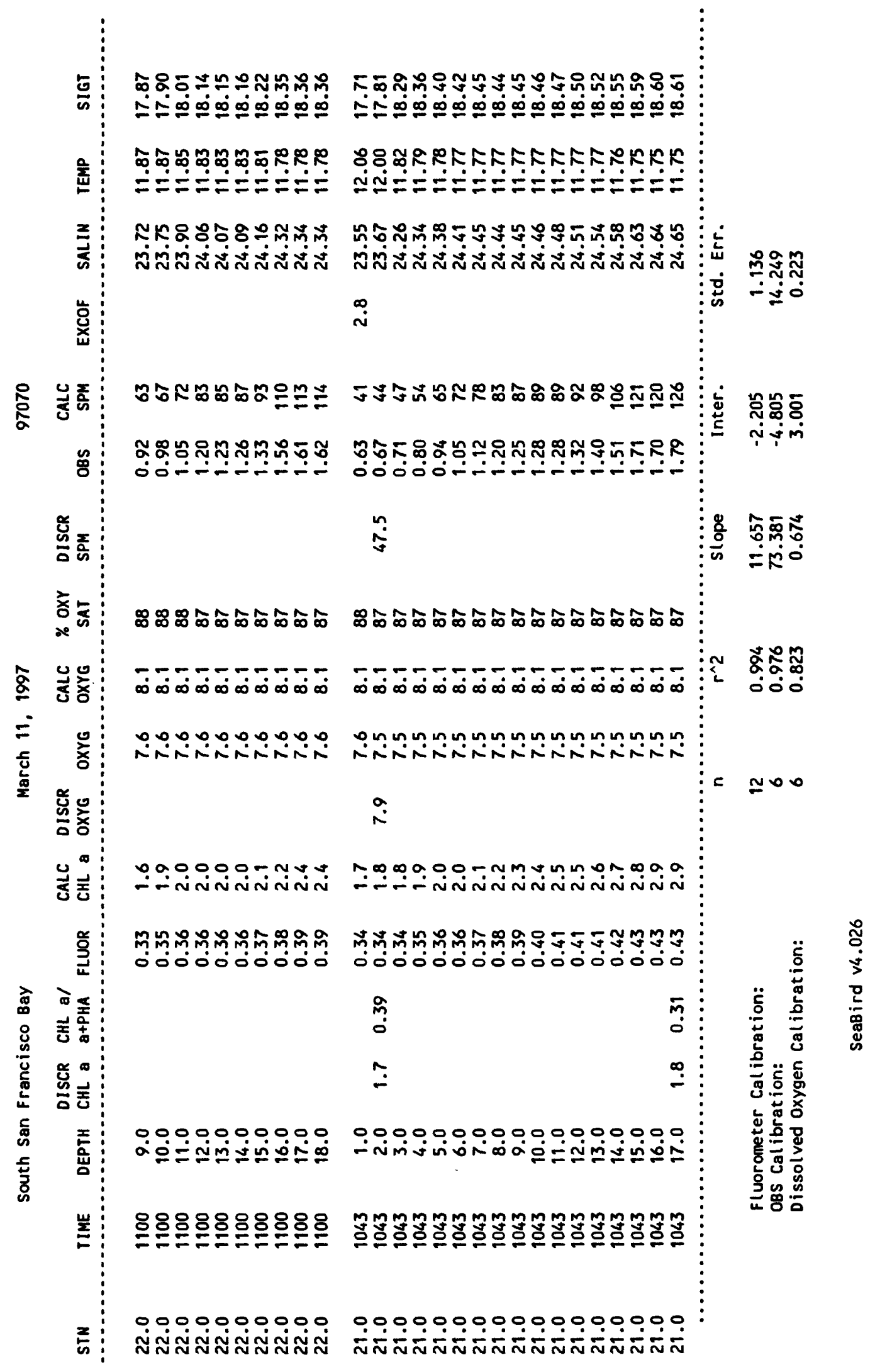




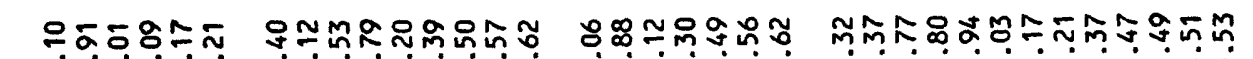

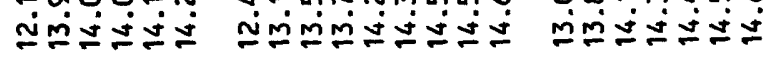

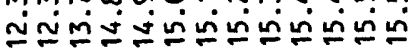

ำฺ

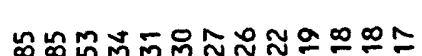

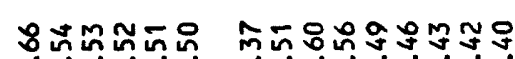

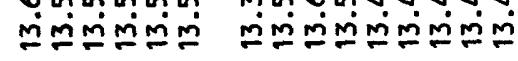

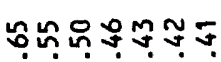

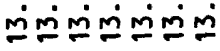

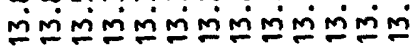

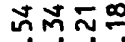

mต̣m

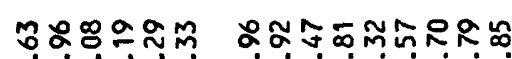

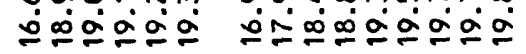

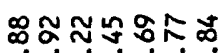

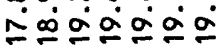

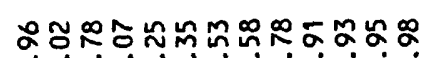

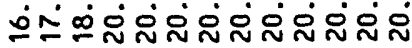

m조

$\stackrel{\circ}{\dot{m}}$

ì

ํํํำ

$\stackrel{0}{\dot{j}}$

$\stackrel{\circ}{\sim}$

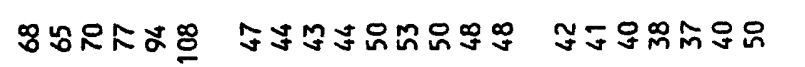

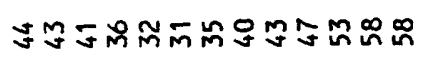

NָN

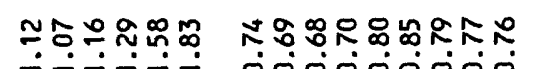

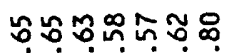

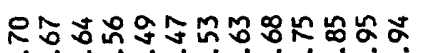
ơ 0000000000

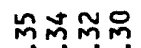
०0.0

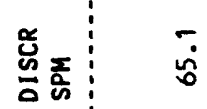

in

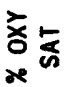

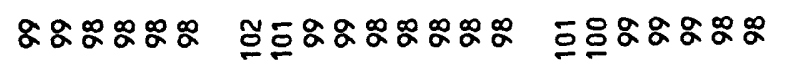

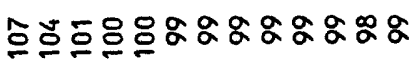

$\ddot{\sim}$

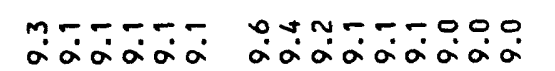

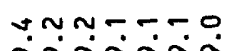

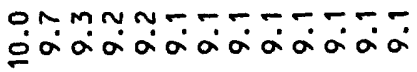

드융ㅇ

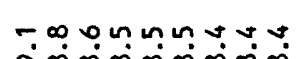

$\infty 00 \ln n \min =$

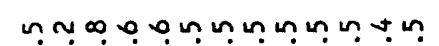

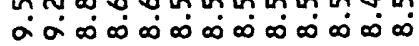

mฺ̃ó $\infty \infty \infty \infty \infty \infty$ $\infty \infty \infty \infty \infty \infty \infty \infty$

$\infty \infty \infty \infty \infty \infty$

T.0.0 $\infty \infty_{\infty}^{\infty} \infty \infty^{\circ}$

$$
\stackrel{\infty}{\infty}
$$

㝴志

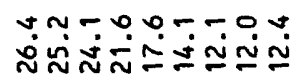

mm?nma-

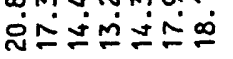

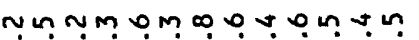

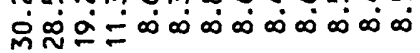

$$
\infty
$$

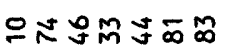

\section{용ํํำำ}

ธินีกษ์

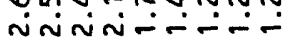

iัํำ-口

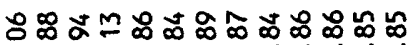

min- i0000000

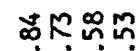

0ं00

0000000000000 


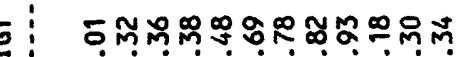
๑ํํํํำำ

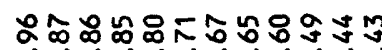
บำำำํำำ

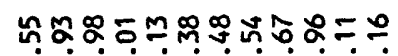

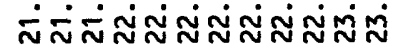

蒙

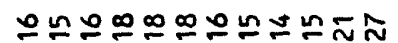

象

总紊

各点

5
$\hat{5}$
$\frac{5}{0}$
$\frac{5}{2}$

过

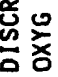

㝴突

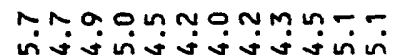

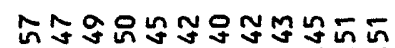
कं000000000

$0909990 \infty \infty \uparrow \uparrow \infty$

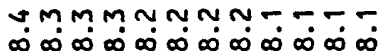

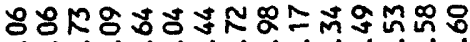

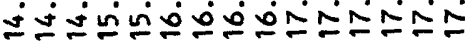

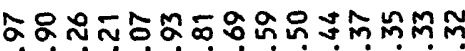

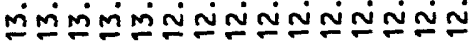

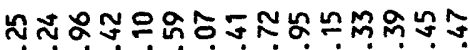

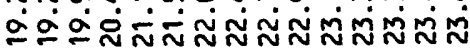
$\stackrel{\circ}{i}$

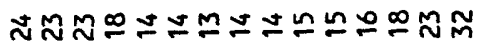

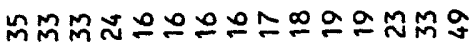
o000000000000

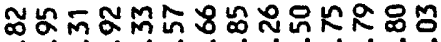

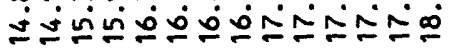

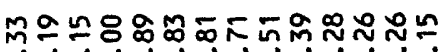

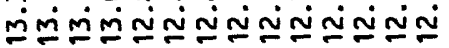

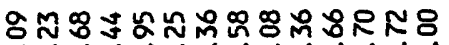

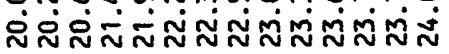

$\dddot{\sim}$

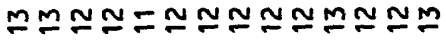

ปปปM 0000000000000

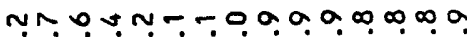

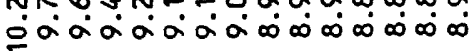

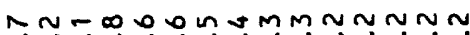
مَّ人 aें

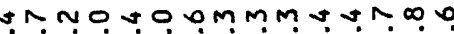

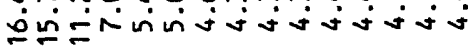

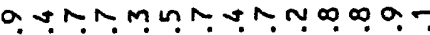

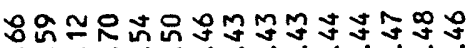
$\because \because 0000000000$

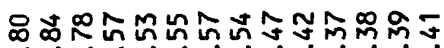
00000000000000

000000000000000000000000000

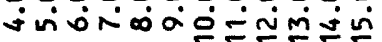

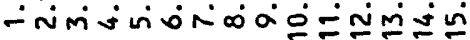

0.000 .000 .000 .000 .0

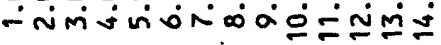

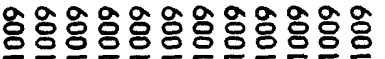

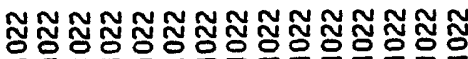

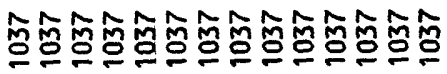




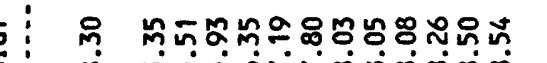

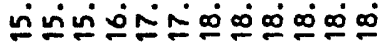

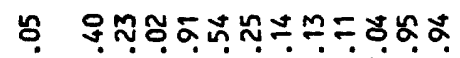

$\stackrel{\sim}{\sim}$

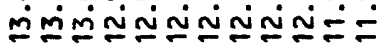

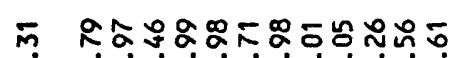

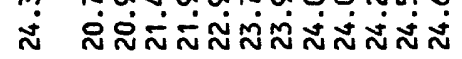

$\div$

苍 巡

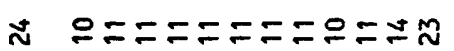

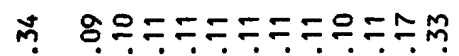

:

递

希产

ล

j

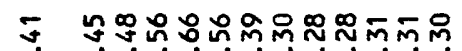

o 00000000000

ก

ำ.

$\stackrel{\infty}{\text { in }}$

$\stackrel{m}{i}$

옹

:

$\stackrel{0}{\circ}$

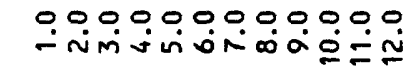

놀

ติ

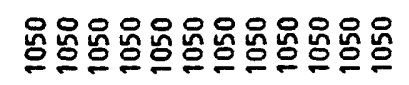

0000000000

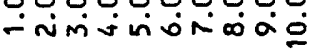

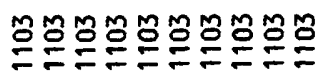

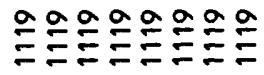

00000000 - $\sim$ mínín

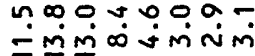

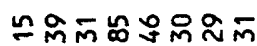
$\therefore 00000$

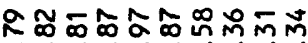
0000000000

NNナaN00, वंवंळ்

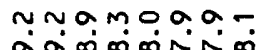

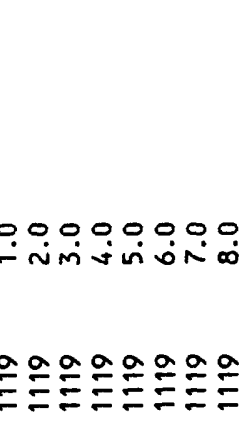

$0.0010 \% 0 \%$

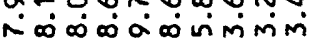

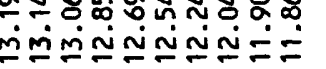

ஸி

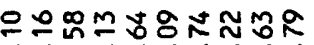

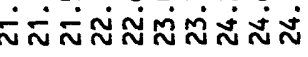

$\dddot{-}$

$M M M \simeq \simeq \simeq \simeq 0 ㅇ$

-0000000000

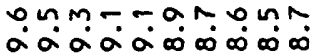

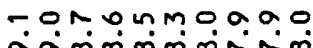

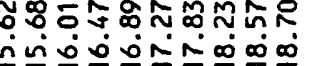

吢出品的尔品

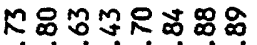

กำํํ요

ำㅇำ Mญニニ

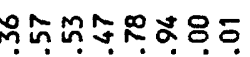

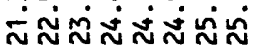<smiles>[CH]</smiles>

으으응ニㄴํㅇ

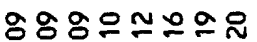
०0000000

$\stackrel{\sim}{a}$

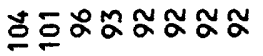

ง a $\dot{0} \infty \dot{\infty} \infty \dot{\infty}$

$0, \sim 0 \infty \infty \infty \infty$ $\infty \infty \dot{\infty} \dot{\infty} N \wedge$

$$
\tilde{a}
$$

$\sim \infty a a \sim \infty \sim 0$ $\infty \infty_{0} \dot{0} \operatorname{inn} \tilde{n}$

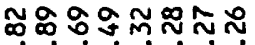
00000000

$$
\begin{aligned}
& \tilde{\alpha} \\
& \dot{0} \\
& \dot{0}
\end{aligned}
$$

00000000 - imivivio

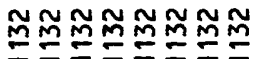
0.00000000

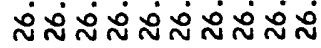

00000000

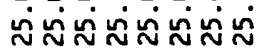

00000000

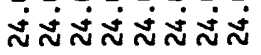




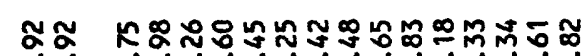

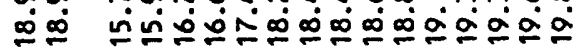

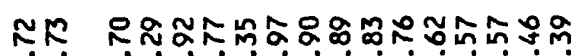

立

ปัర

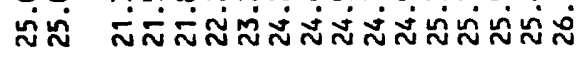

$\stackrel{m}{m}$

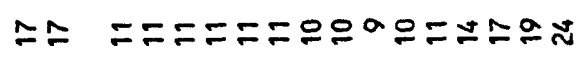

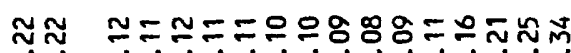

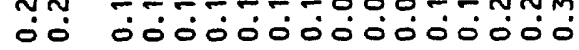

官

oo no-arm onnorao

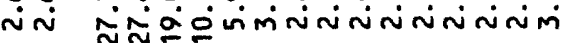

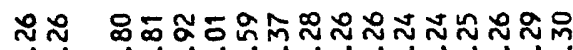

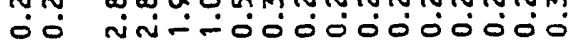

:ִ

$\stackrel{0}{\circ}$

00.000000000000000

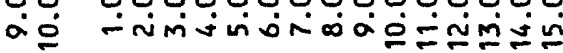

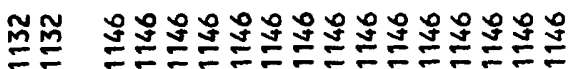

กัं

0.0000000000000

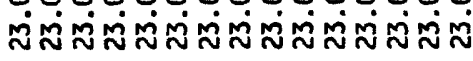

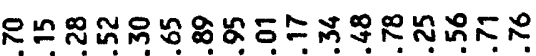

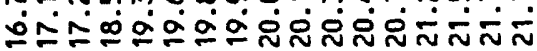

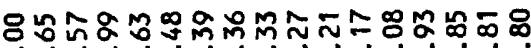
mํ்

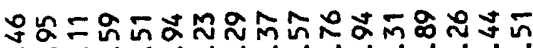

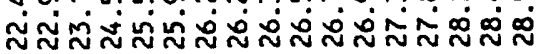

$\ddot{0}$

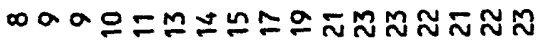

ニニニニニニ

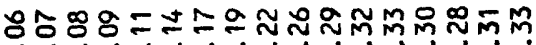
0000000000000000

ํニニニ= 000000

$\stackrel{a}{\cong}$

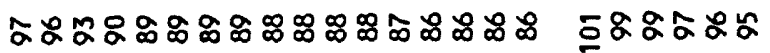

m

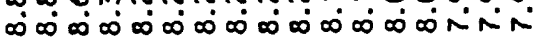

$4 \div-0.0 \infty$

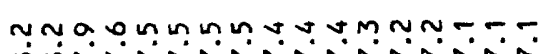

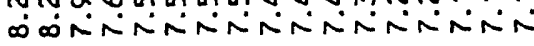

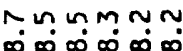
$\ddot{a}$

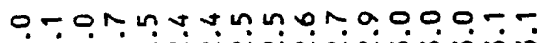
mimiñiñNiñiñmimmim

ज̛̣mimimi

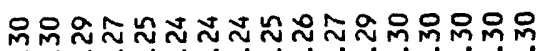
000000000000000

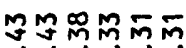
000000

$$
\begin{aligned}
& \stackrel{0}{0} \\
& -\dot{0}
\end{aligned}
$$

00000000000000000 -

000000 - imivisio

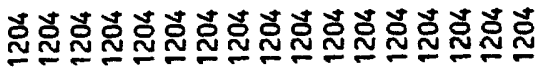

$\stackrel{n}{\frac{n}{n}} \frac{n}{\frac{n}{N}} \frac{n}{N} \stackrel{n}{\frac{n}{N}}$

00000000000000000 สำกำก
0.000 .00$. 玄玄市市 


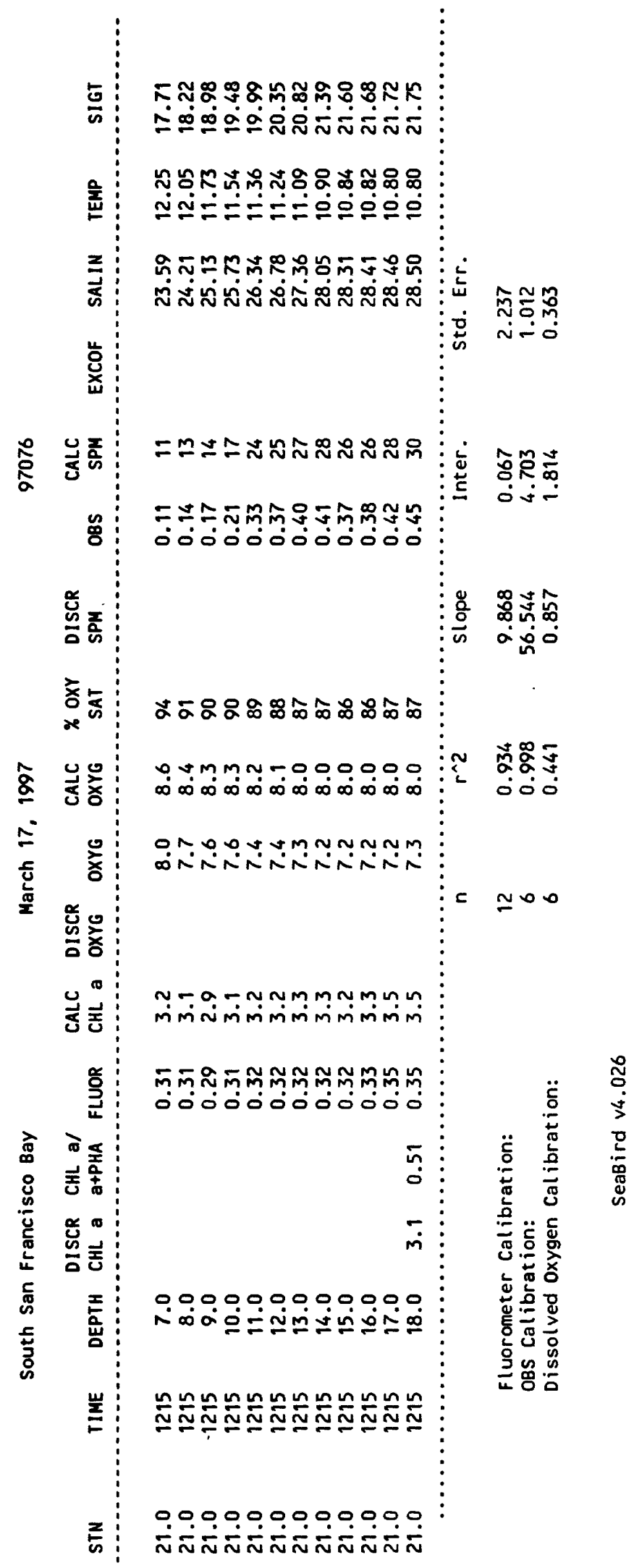




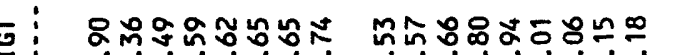

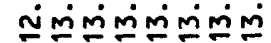

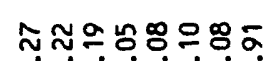

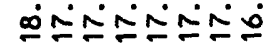

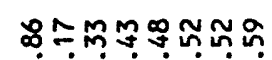

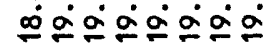
:

总

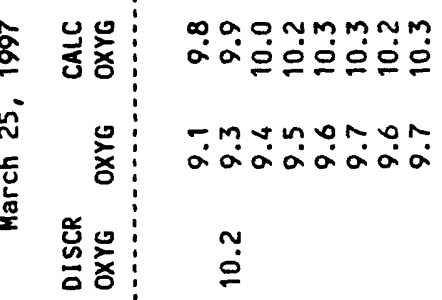$$
\text { ๗ั }
$$$$
\text { 壳 }
$$

造至

总

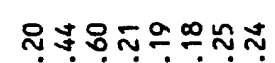

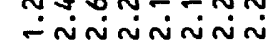

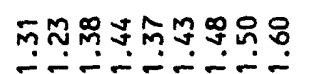

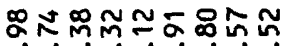

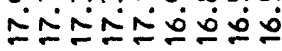

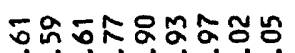

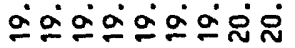
in ณั่

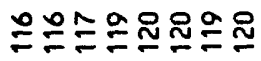

n nm- บาง

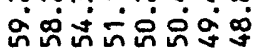

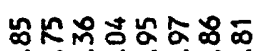

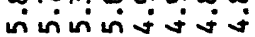

$\stackrel{\infty}{?}$

ó

$?$

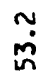

ผั่

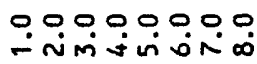

돈

訔

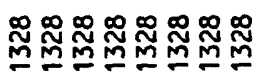

00000000 m̊nmmm

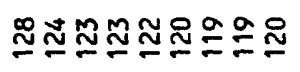

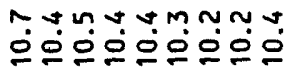

พ⿻․․

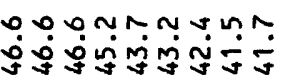

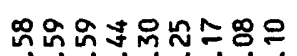

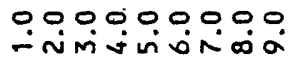

음으뭉aㅁㅇaㅇ

000000000

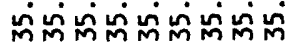

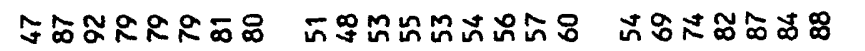

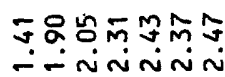

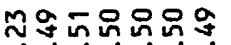

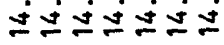

ธกลณณณぇ

ஸீㅇํㅇㅇำ

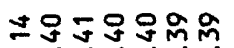

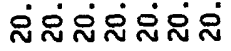

m.

$m$

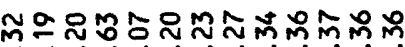

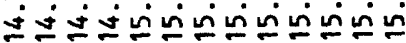

ธณ ஸ்

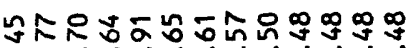

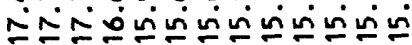

$\hat{a} \div$

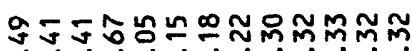

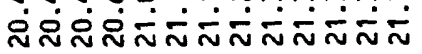

8ํ. ஸ் $\stackrel{a}{\sim}$

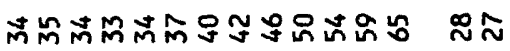

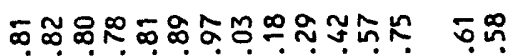

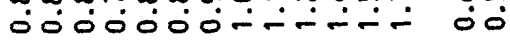

$\stackrel{n}{m}$

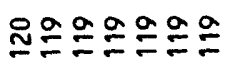

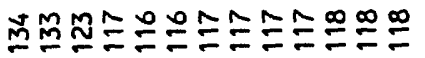

$\stackrel{\mathbb{N}}{\sim}$

MmMmMmm

MNMO- N N NMMMM

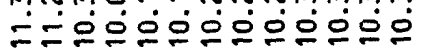

$\because 0$

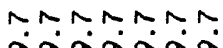

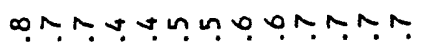

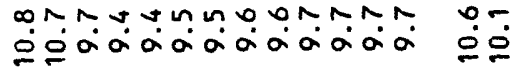

$\stackrel{+}{9}$

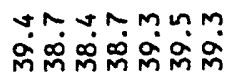

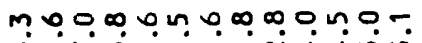

똘

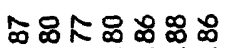

miniminimin

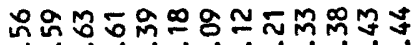

$\stackrel{2}{2}$

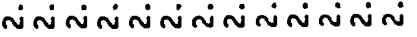

F $\stackrel{9}{i}$

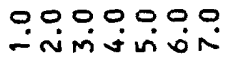

0000000000000

$\stackrel{0}{-i}$

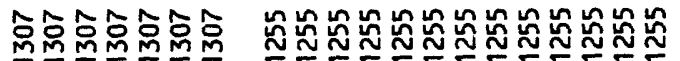

莣

0000000

0000000000000

0.0 mंजिंलेंजि

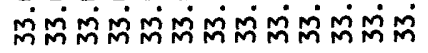

लंก 


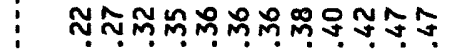

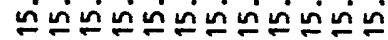

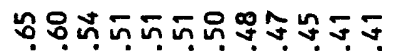

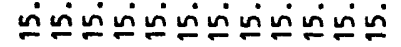

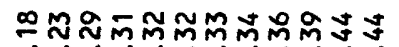

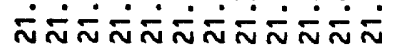

悹

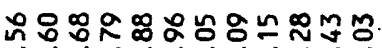

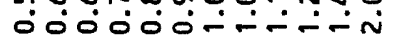

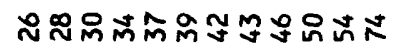

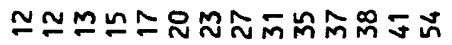

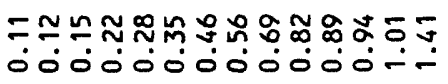

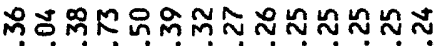

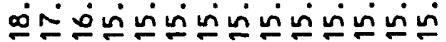

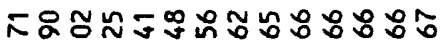

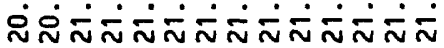

$\cong$

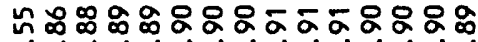

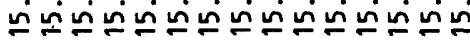

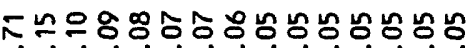

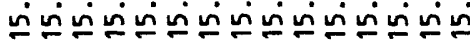

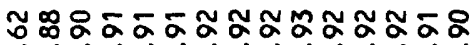

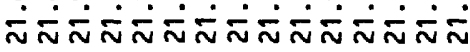

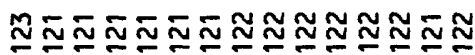

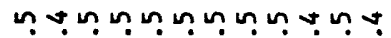

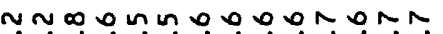

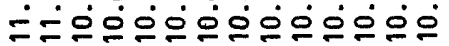

TOOONNNNNNNNNN aagaaaaaaaaa

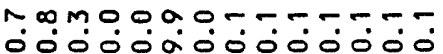
으웡ㅇㅇㅇㅇㅇㅇㅇㅇㅇㅇㅇ

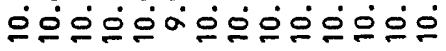

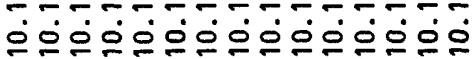

$$
\text { ก̊ำ }
$$

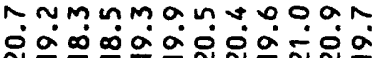

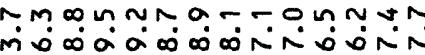

mammnvan-mo-via

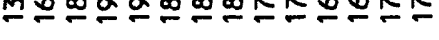

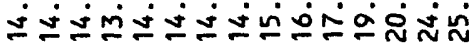

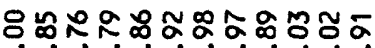

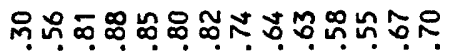

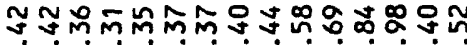
n-.-.-.

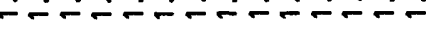

$\stackrel{m}{\leftrightarrow}$

00000000000000

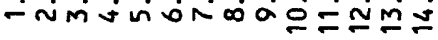

-

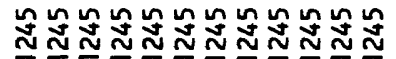

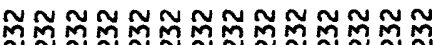

nกำ ミミミミミミミミミミミミミミミミミ 


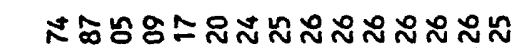

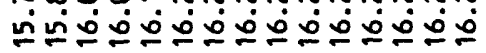

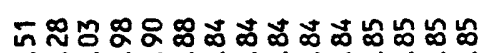

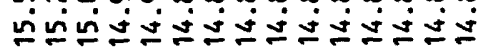

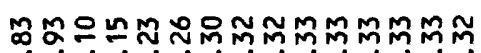

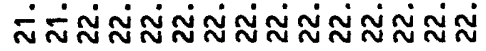
?

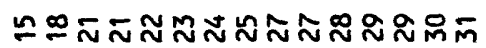

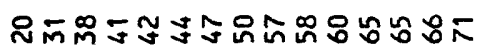
ठ000000000000

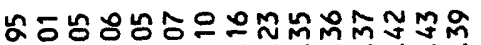

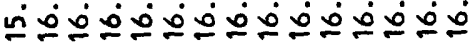

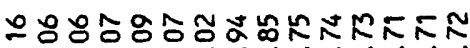

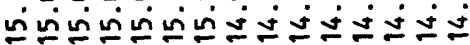

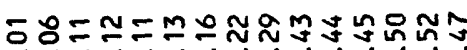

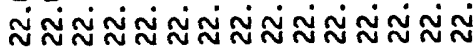
$\stackrel{m}{9}$

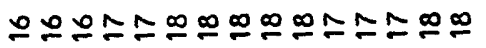

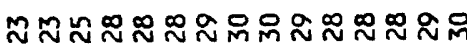
ல000000000000

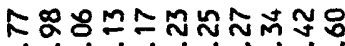
ஸ்

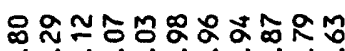

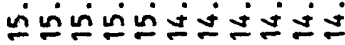

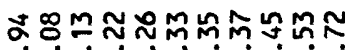

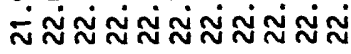
o.

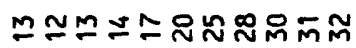

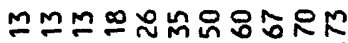
O0ं0000000

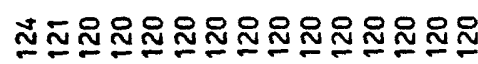

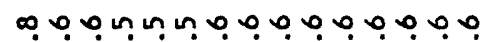

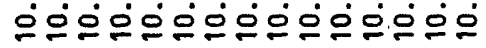

m00000000000000

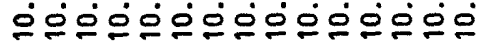

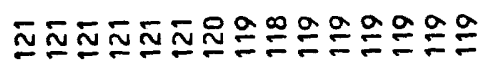

0 0 0 0 o onn 응ㅇㅇㅇㅇㅇㅇㅇㅇㅇㅇㅇㅇㅇㅇㅇ

$0-7-0.000090 .9000$

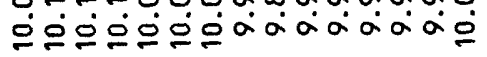

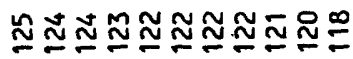

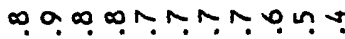
웡ㅇㅇㅇㅇㅇㅇㅇㅇ mmmm $m \sim n-7 a$

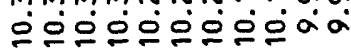

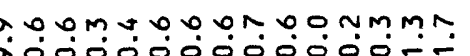

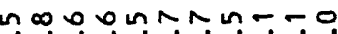

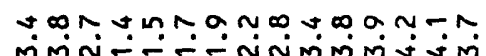

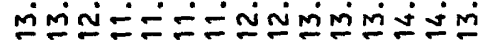

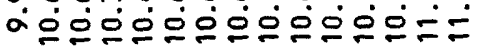

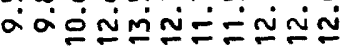

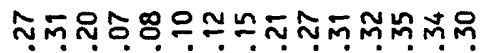

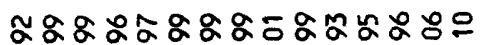

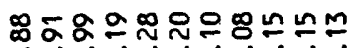
0000000ั0000-

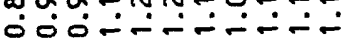

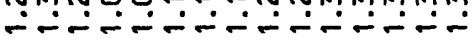

0.0000000000000 -

000000000000000

00000000000

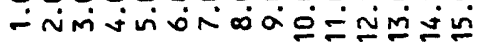
- imivinisosio

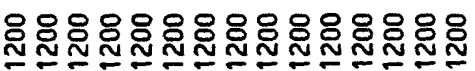

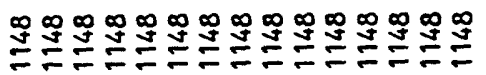

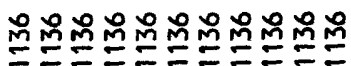




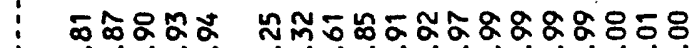

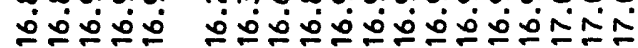

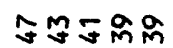

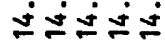

กิ่ที่ก

总

苋 䞤

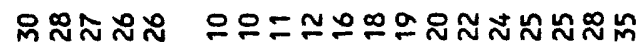

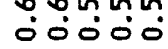

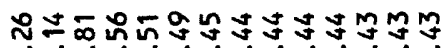

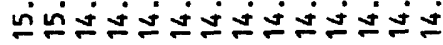
웟ำ

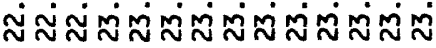

$\stackrel{\infty}{0}$ 0000000000000
뜽ㅇㅇㅇㅇㅡ

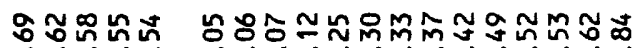

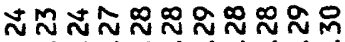

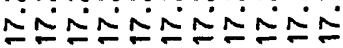
$\stackrel{\circ}{i}$

\section{$\stackrel{\infty}{=} \stackrel{0}{=} \frac{0}{=}$}

nn $\ln \operatorname{nn}$ ㅇํㅇㅇㅇ 00000

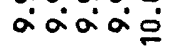

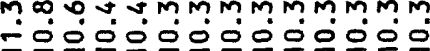

$\stackrel{?}{=}$

m!

岁폰

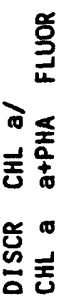

$$
\begin{aligned}
& \text { 品 } \\
& \text { ํํํ }
\end{aligned}
$$

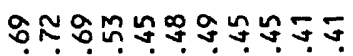

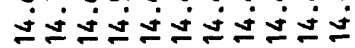

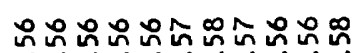

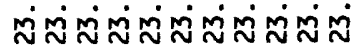

\section{$\stackrel{\square}{-}$}

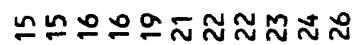

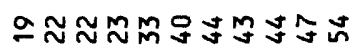
0000000000

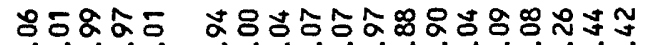
$\because 00 \div$

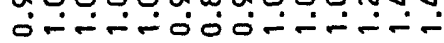

$\stackrel{\substack{0 \\ 0}}{0}$

$\check{1}$

$\stackrel{a}{\infty}$

00000

ํํำํำ

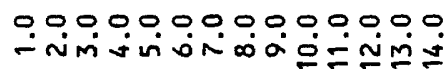

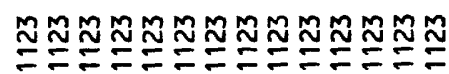

0.000 ณ
00000000000000

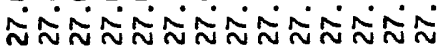

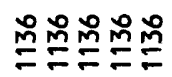

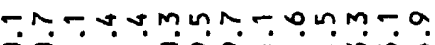

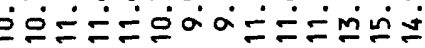

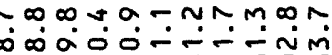

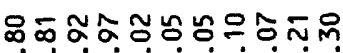

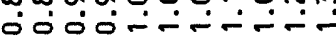

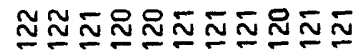

NTO00000000

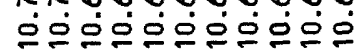

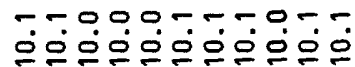

00000000000

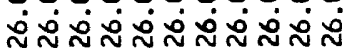

$\mathscr{B} 0$

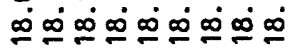

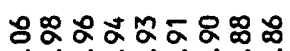

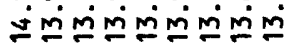

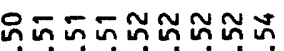

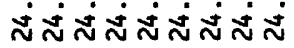
$\stackrel{9}{-}$

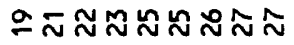

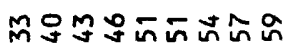
ல0ं0ं0்0ं

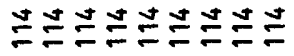

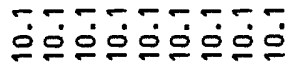

$\cos \theta a d a t a n$

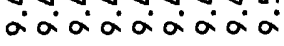

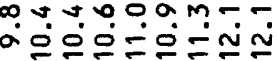

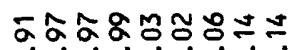
0000영
00000000000

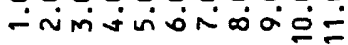

0000000000 - Nmivína

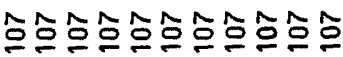

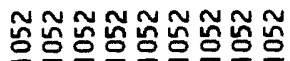

000000000

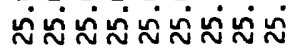


エธดКKNNKNNN

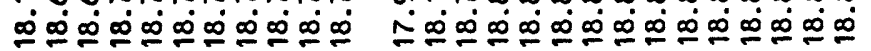

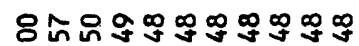

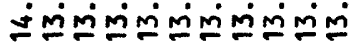

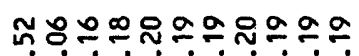

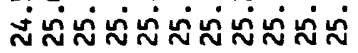

$\stackrel{\infty}{-}$

丞

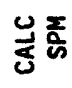

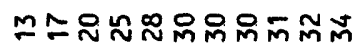

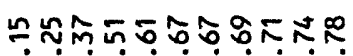

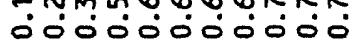

กับ

음

ชิธ

ڤ

๗ं

娄

을

옹롱

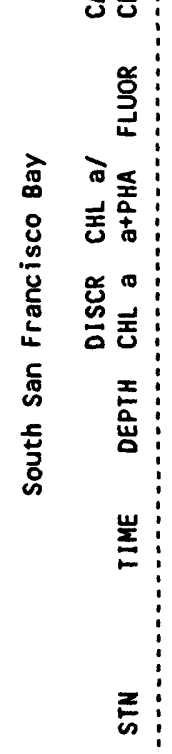

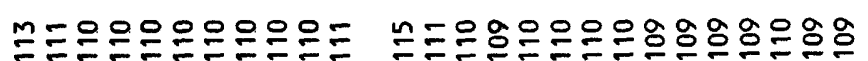

$\alpha \infty \infty \infty \infty \infty \infty \infty \infty \infty$

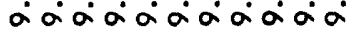

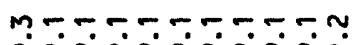

aरaबaरaकaara

$\check{0}$

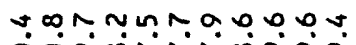

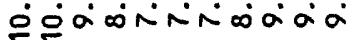

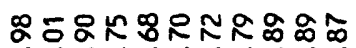
Оं0ं0ं0000

ळ.

$\stackrel{0}{\vdots}$

$\dot{\square}$

$\stackrel{+}{\infty}$

00000000000

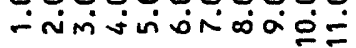

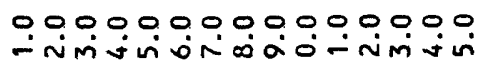

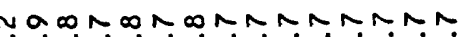

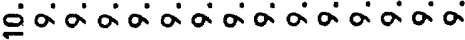

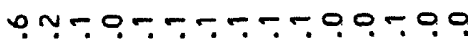

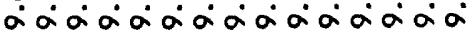

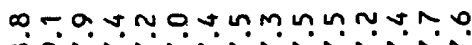

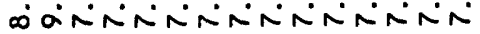

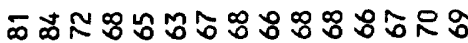

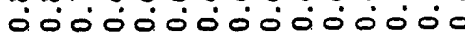

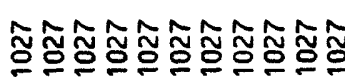

0.000 .000000

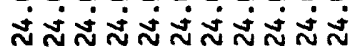

000000000000000

$\mathbb{N} \mathbb{N} \mathbb{N} \mathbb{N} \mathbb{N} \mathbb{N} \widetilde{N} \mathbb{N} \mathbb{N} \widetilde{N}$

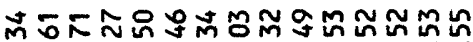

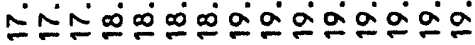

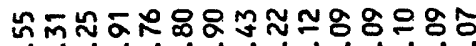

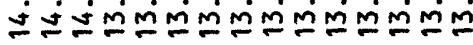

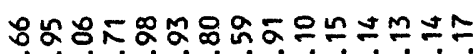

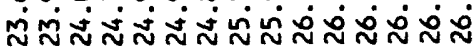
$\dddot{\square}$

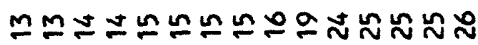
ปำลำสำตำำกำกำ

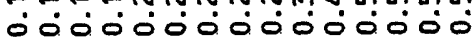

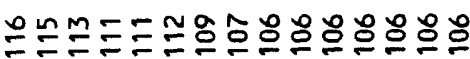

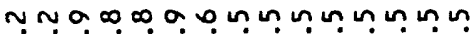
ㅇaaaaaaaa

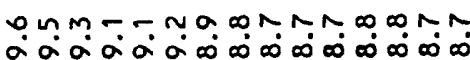

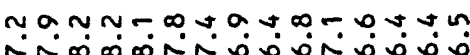

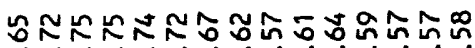
0000000000000

000000000000000

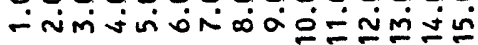

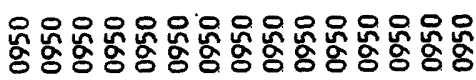

000000000000000

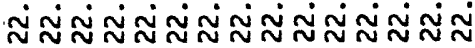




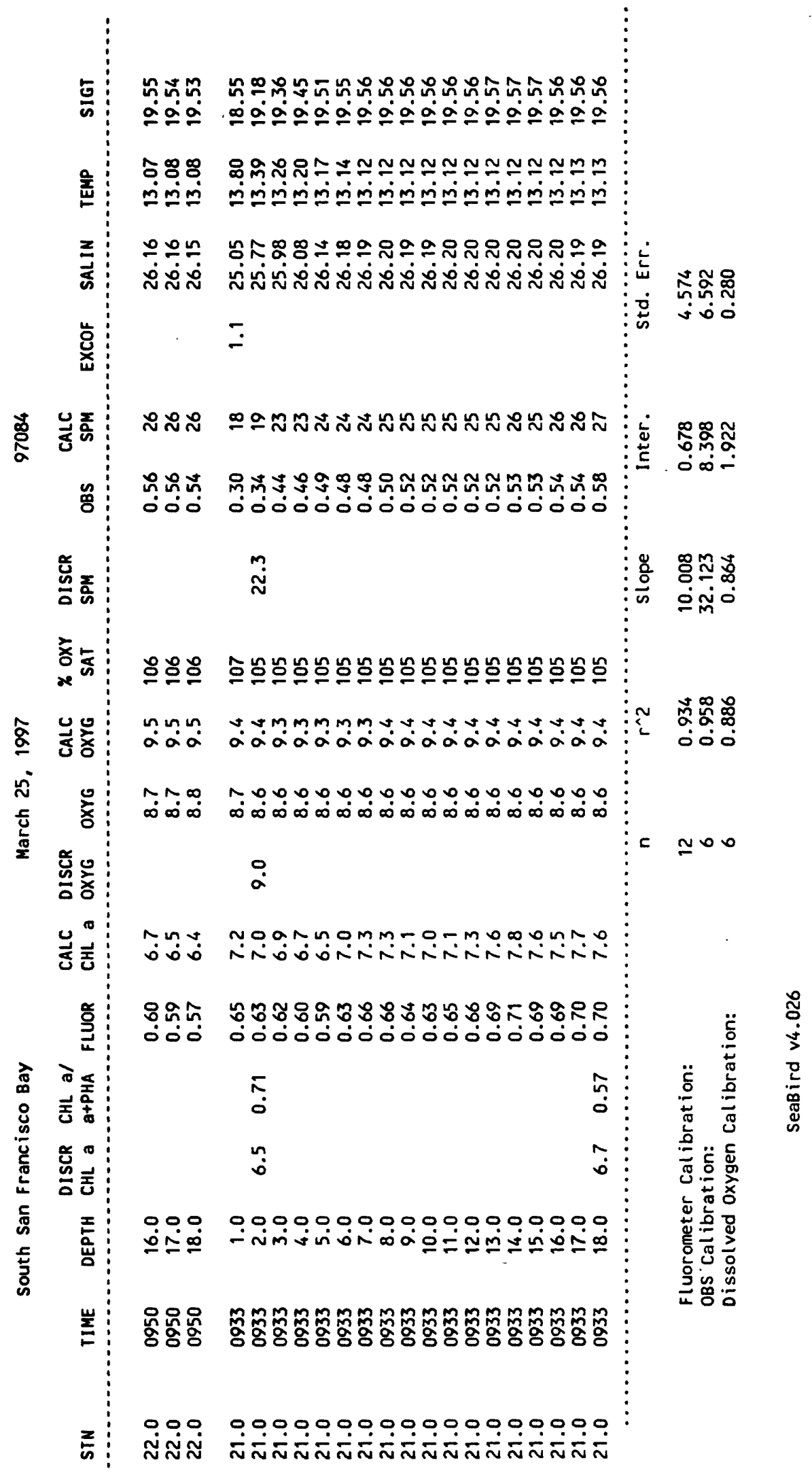


8우우우우우웅

०00000000

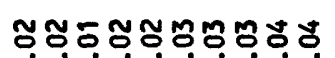

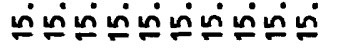

mMMMำ

0000000000

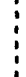

容

通要

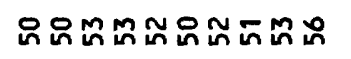

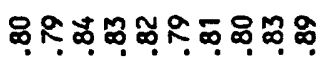

0000000000

䓵

$\stackrel{m}{\infty}$

爻禀

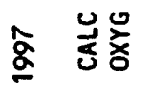

$=0$

颉

놇론

000.090 .090

ajajacaja

mMmmMmMmmm

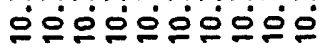

$$
\stackrel{0}{\circ}
$$

ทำ

NRNNNDDNN

22ㅇํㅇํํㅇํำ

000- - -

:े

ڤัo

$\stackrel{n}{\dddot{n}}$

a

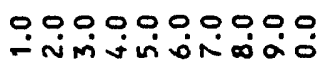

종

崖

루

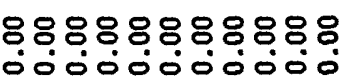

历̄

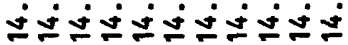

영영영영

ó0000000

$\stackrel{i}{\text { i }}$

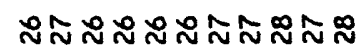

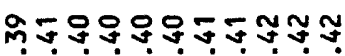
0000000000
88888888888
00000000000

영ㅇㅇㅇㅇㅠேニ

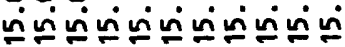

영영영영영 ०000000000

$\stackrel{a}{i}$

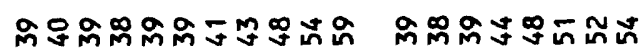

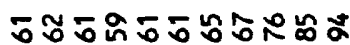
000000000

88888888

๓ำก ำกำ ที่บบที่ที่อ

옹응으으으으으으 00000000

$\stackrel{n}{\sim}$

$88.8 K \square-\infty$ 00000000 0000000

ì.

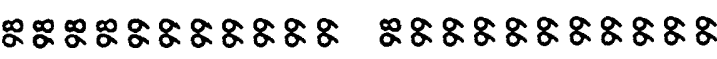

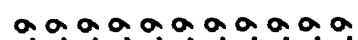

$\infty \infty \infty \uparrow \infty \infty \infty$

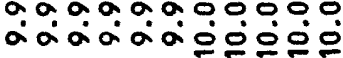

ajaiajajaia

åååå

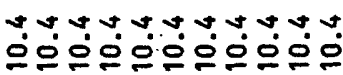

$\stackrel{0}{\circ}$

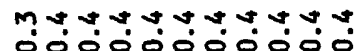

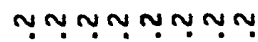

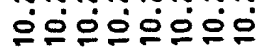

aे

TrN? $900=\overline{0}$

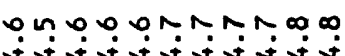

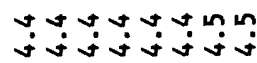

B O०00000000

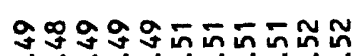
0000000000

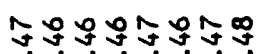
00000000

นกำ

$\stackrel{\circ}{m}$

00000000000

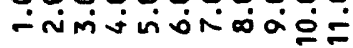

00000000 -nimín

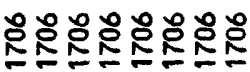


8888888888888888888

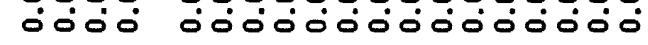

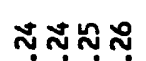

ที่ยู่อ

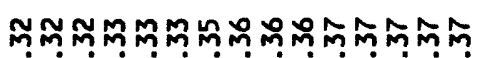

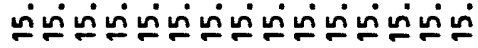

오으으으 으으으으으으으으응ㅇㅇㅇㅇㅇㅇㅇㅇㅡ

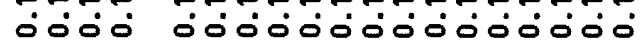

กุ

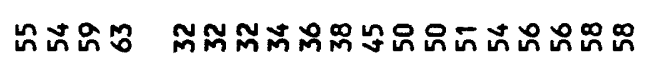

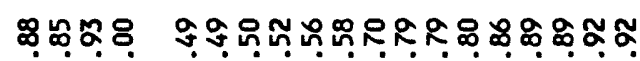

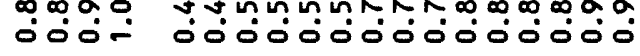

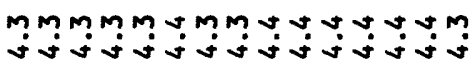

ษิงก๊ ๑ं。்

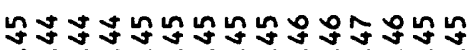
0000000000000

00000000000

$m$
8888888888880

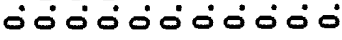

旅员的的的的的的

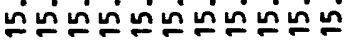

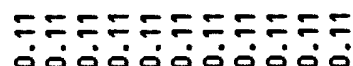

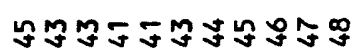

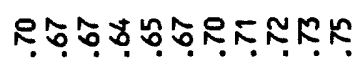

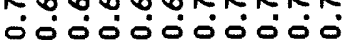

8888888888

000000000

RKRKNNNNRK

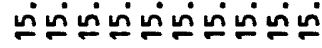

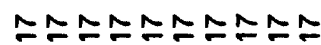
000000000

$\stackrel{9}{9}$

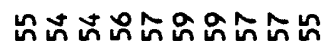

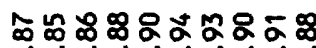
0ं0ं000000<smiles>[AlH2]</smiles>

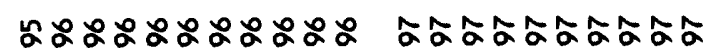

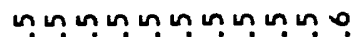

(2000 aरajacasa

9.9 .9 .9 .9 .9 .9 .9 .90

0000000000

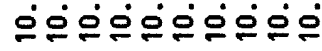

\section{ณ?}

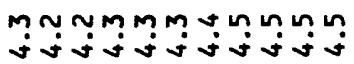

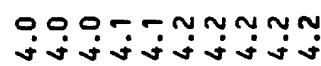

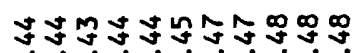
व0ं0ं00000

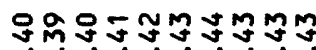
कीं000000

:

ợ

$\stackrel{\circ}{m}$

$\hat{m}$

0000000000

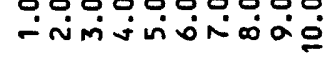

$\infty \propto \infty \infty \infty \infty \infty \infty \infty$

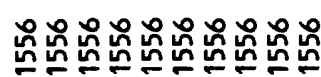




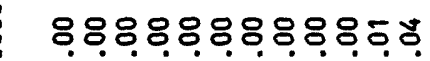
00000000000

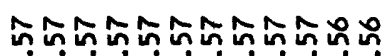

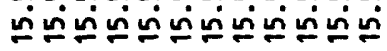

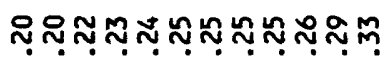

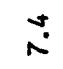

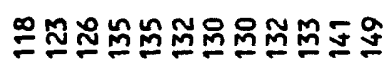

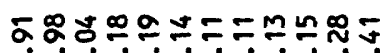

$\because$ iñninininiña

莒

爻乱

ล

$=0$

웅 웅

迆

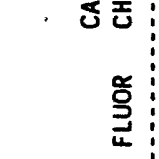

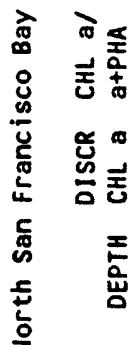

崖

告:

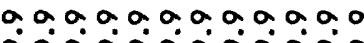

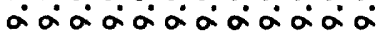

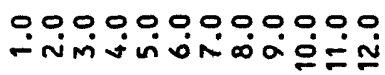

0.0000000000 ninininising

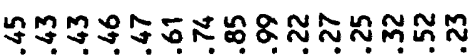

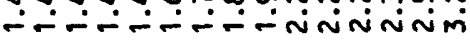

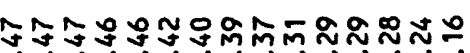

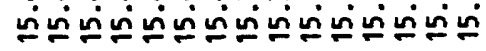

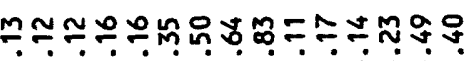
miniminiminimis $\stackrel{\infty}{\infty}$

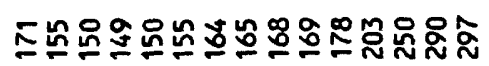

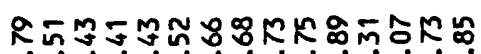
Niñiñññññmist

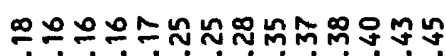
miniminiminiminiminiminim

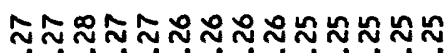

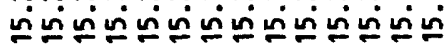

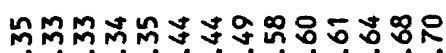

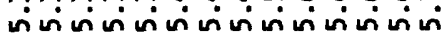

$\bar{\infty}$

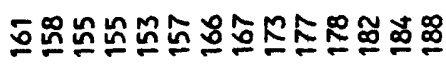

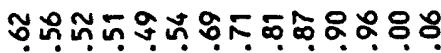
NiñNiñNinininimim

กิ

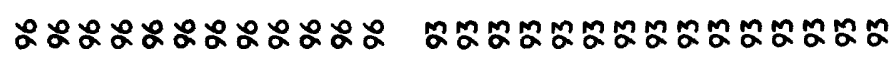

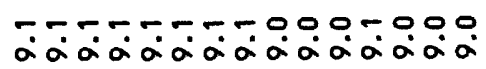

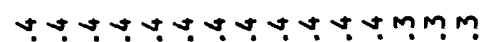

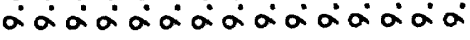

un un un un un un un un un $\alpha \alpha \alpha \alpha \alpha \alpha \alpha a \alpha \alpha \alpha a \alpha$

$$
\bar{a}
$$

00000 nnmatanuming

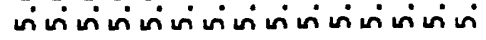

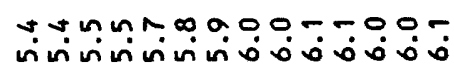

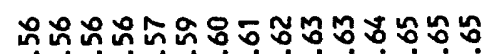

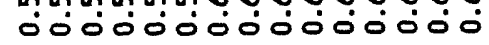

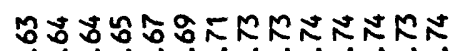
O0000000000000

$\stackrel{9}{\circ}$

in

0.00000000000000

0.000000000000 -

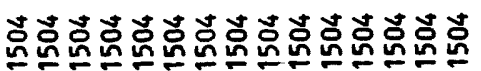

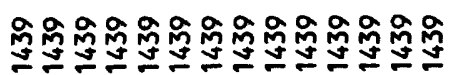

000000000000000

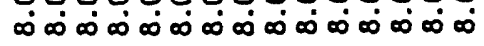

00000000000000 ááááááááá 


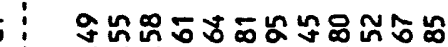

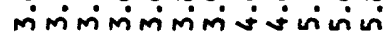

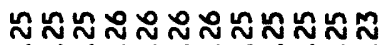

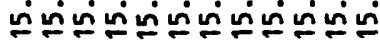

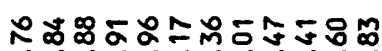

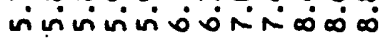

䒝

จ

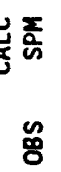

荾

ชัธิ

๙

赵동

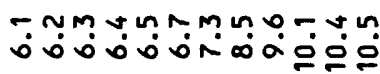

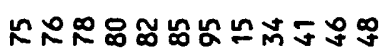

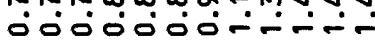

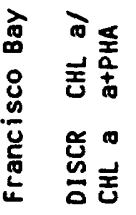

હ

홍

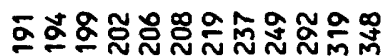

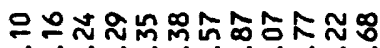

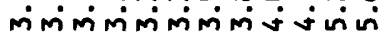

?

ì

0.00000000000

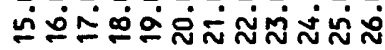

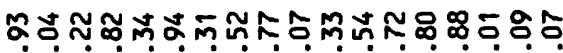

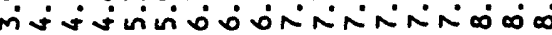

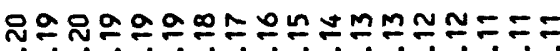

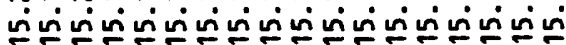

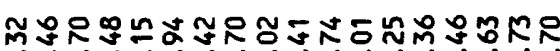

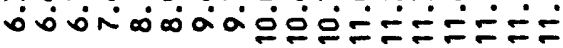
$\stackrel{\infty}{\infty}$

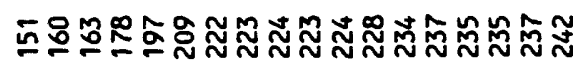

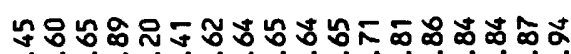

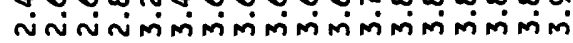

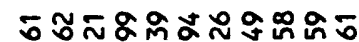

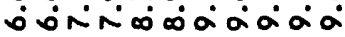

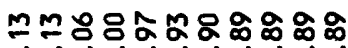

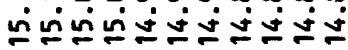

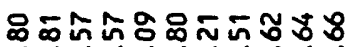

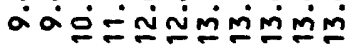

$\overline{0}$

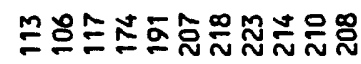

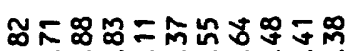
-

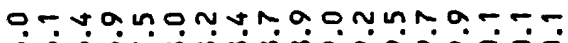

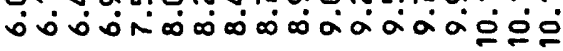

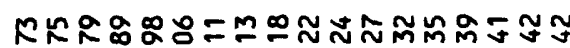

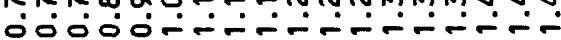

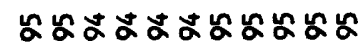

$00, \infty \infty \infty \infty \infty \infty \infty \infty$ $\dot{\infty} \infty \dot{\infty} \infty \dot{\infty} \infty \dot{\infty} \infty \dot{\infty} \infty \dot{\infty} \infty \dot{\infty}$

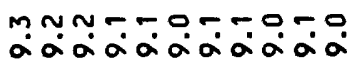

0.00000000000000000

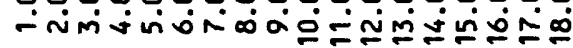

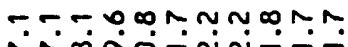

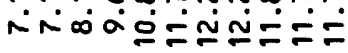

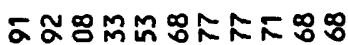

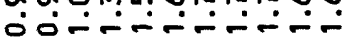

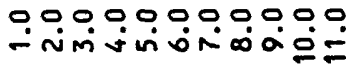




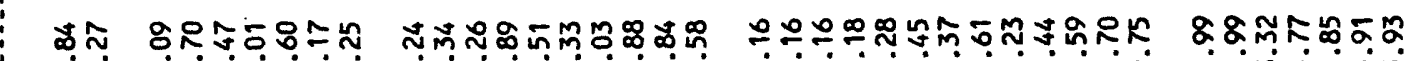

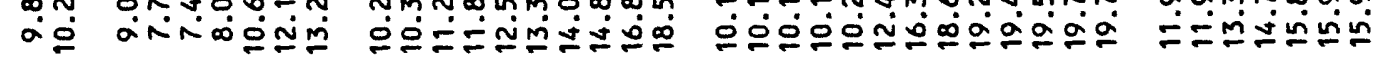

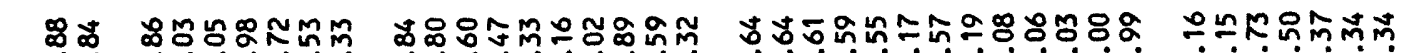

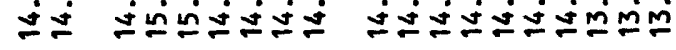

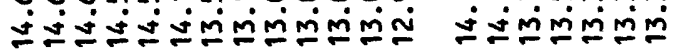

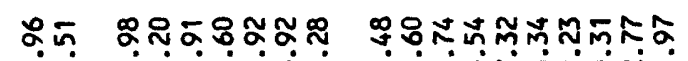

Mป

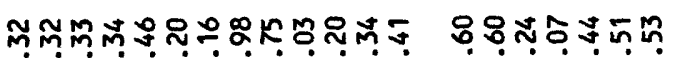

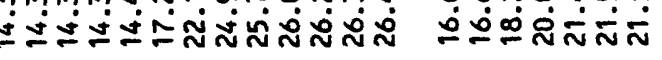

:

$\stackrel{0}{i} \quad \hat{i}$

$\dot{m}$

$\stackrel{\infty}{m}$

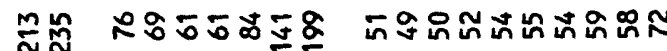

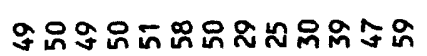

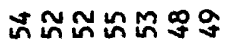

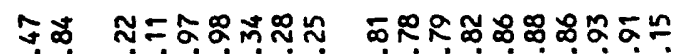

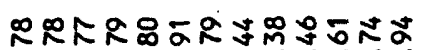

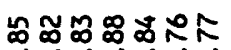

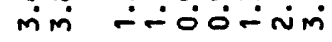

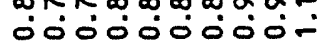

0000000000000

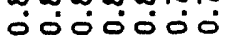

$\underset{\mathfrak{q}}{\mathfrak{q}}$

$\bar{n}$

呚

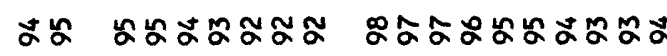

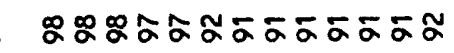

๖゚ล゚ลล์ล

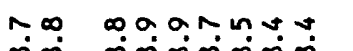

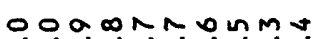

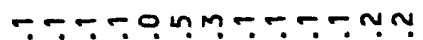

$\div-0.0090$

$\infty \infty \infty \infty \infty \infty \infty \infty \infty$

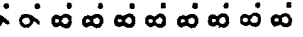

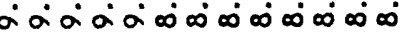

$\alpha \sigma a \infty \infty \infty \infty$

OO -n-ONOO mmn-Oagnuno

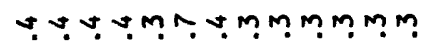

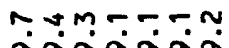

음

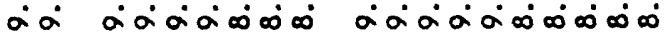

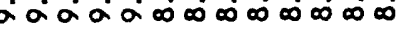

$$
\stackrel{\circ}{\circ}
$$

m.

范芒

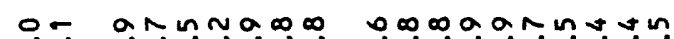

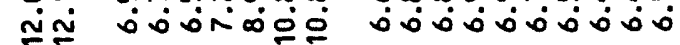

nn n.

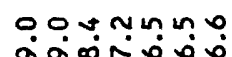

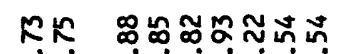

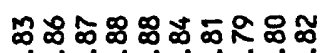

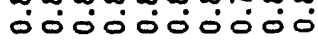

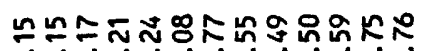

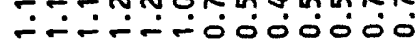

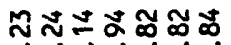

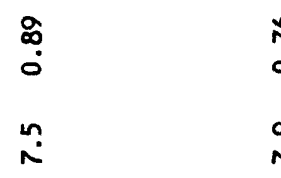

$\stackrel{0}{\circ}$

$\stackrel{\infty}{\mathfrak{0}}$

$\infty$

웅롱

$\stackrel{9}{\sim}$

$\dot{+}$

00.00000000000000000

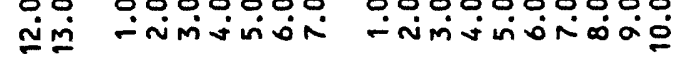

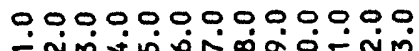

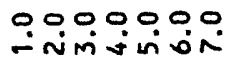

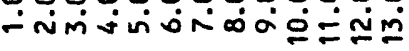

崖

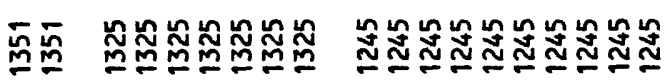

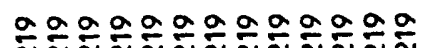

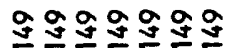

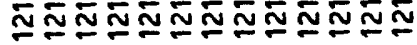

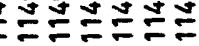

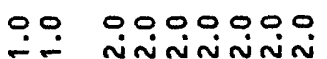

00000000000

0000000000000

0.00000

䒺

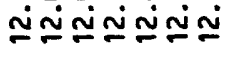

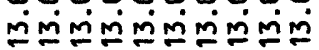

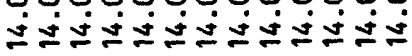

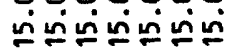




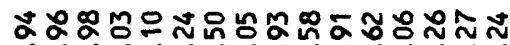

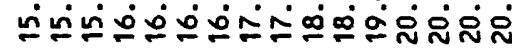

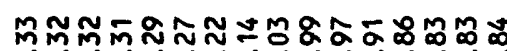

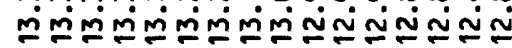

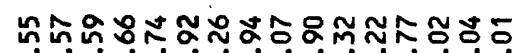

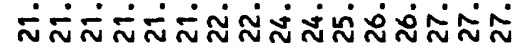

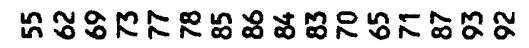

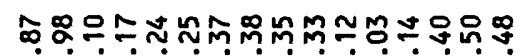

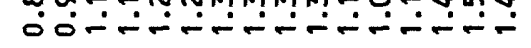

$\infty 0 \infty+m$ m

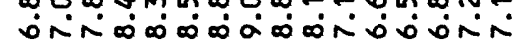

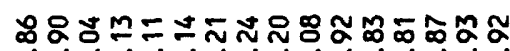

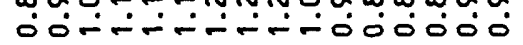

$$
\text { in }
$$

$\stackrel{\circ}{a}$

0,000000000000000

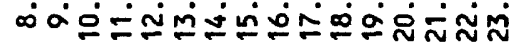

000000000000000 - Nimivionag

0000000000

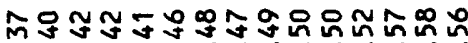
0000000000000

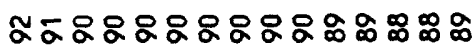

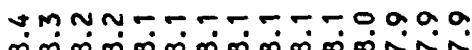

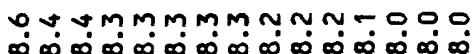

mon-rana o o onoro

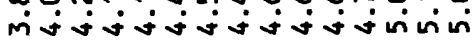

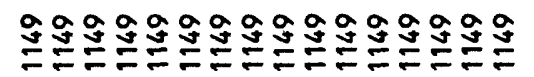

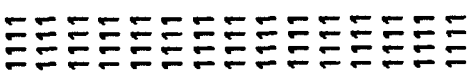

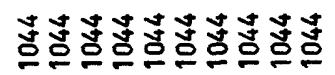
0000000000000000

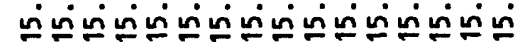

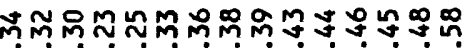
000000000000

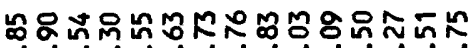

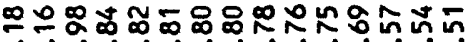

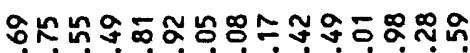

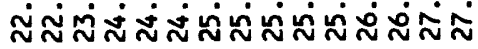
$\dddot{2}$

$\dddot{2}$

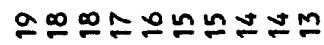

ลกำสกำกำำ -0.00000000 a onNNNNNmígígis

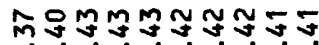
0.0000000

- imivion

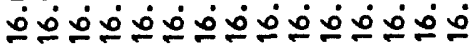

0000000000

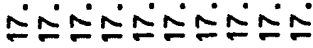




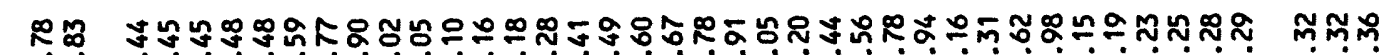

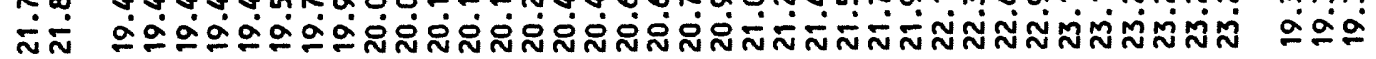

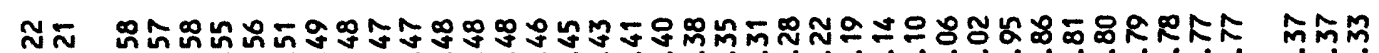

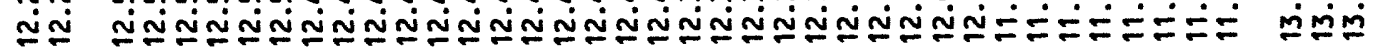

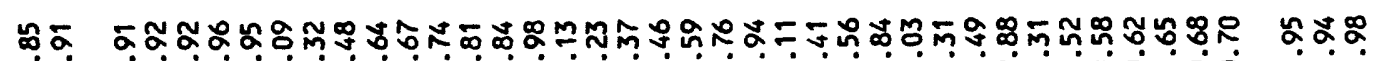

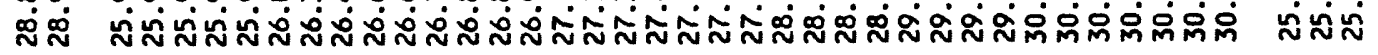

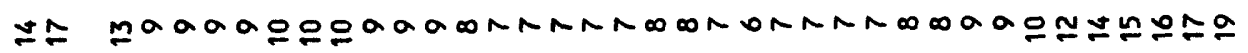
$\underline{\sim}$

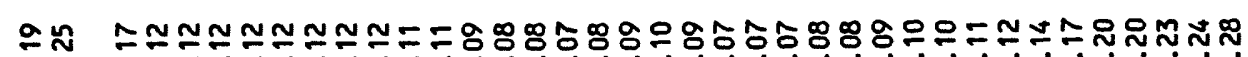

í 0̆

유두 ¿ं०

$\stackrel{\infty}{\infty}$

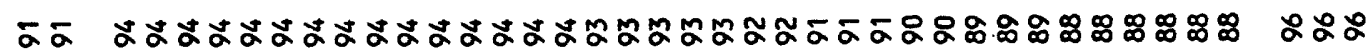

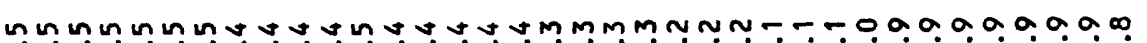

nn

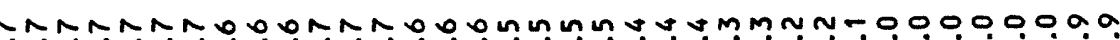
$\infty \infty \infty$

$$
+
$$

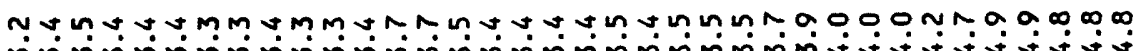

$\operatorname{ioj}^{2}$

$\dddot{\Psi} \overline{0}$

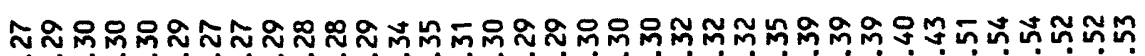

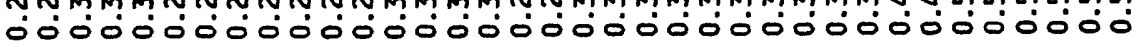

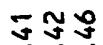

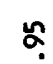

$\dot{0}$ $\hat{i}$ 


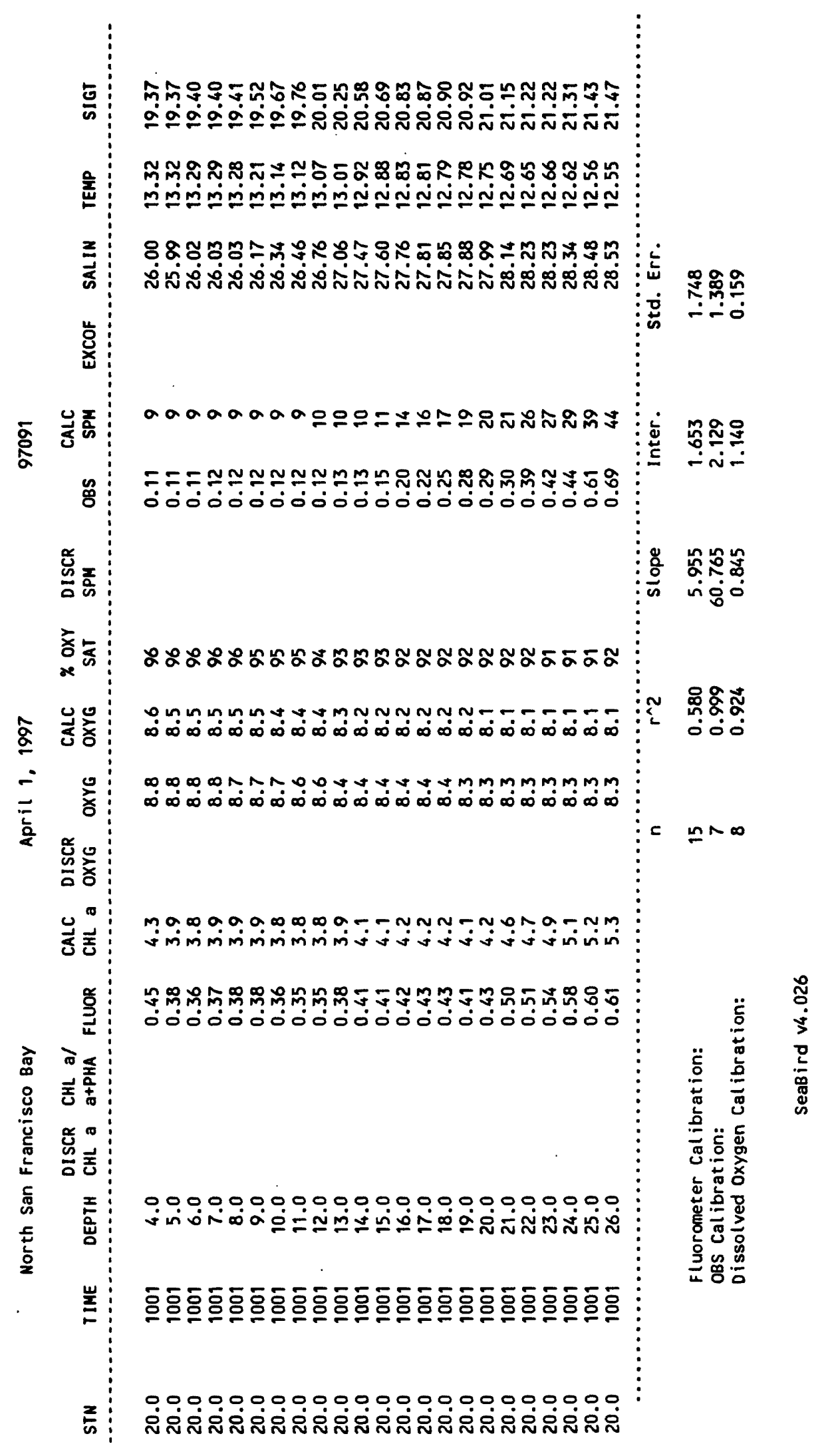




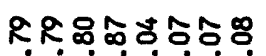

mற்m $\dot{q} \pm \dot{q}$

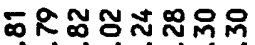

우우ํํํํํํํํํำ

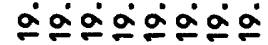

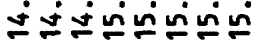

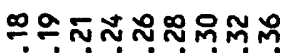

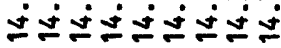

ํㅡณュニำ

ที่ที่ที่ที่อ

Rニペ品以

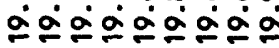

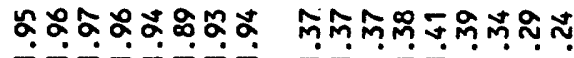

$\stackrel{\circ}{\stackrel{0}{0}}$

ชิ

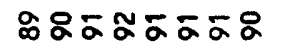

$0-n N-\pi 0$

$\infty \infty \infty \infty \infty \dot{\infty} \infty \dot{\infty} \infty \dot{\infty}$

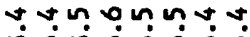

$\infty \infty \infty \infty \infty \infty \infty \infty$

$\stackrel{0}{\infty}$

焉폰

$\checkmark \infty \infty 0$ 0.

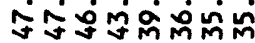

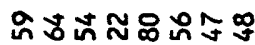

i寸jomimim

$\stackrel{\infty}{0}$

$\stackrel{+}{\circ}$

j

o.

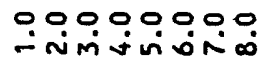

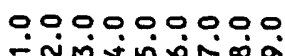

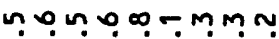

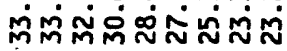

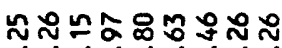
miminininin

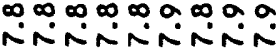

N N N N NMM

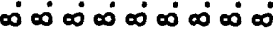

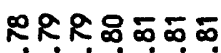

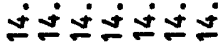

ธํํำำำำ

ที่บทำing

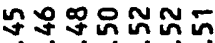

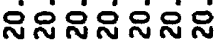

$\stackrel{\infty}{m}$.

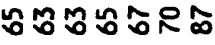

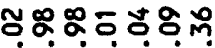

-00

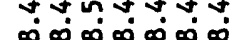

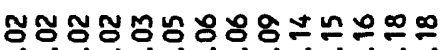

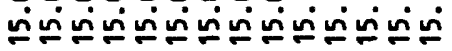

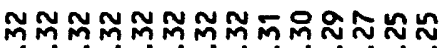

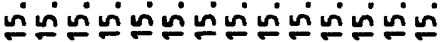

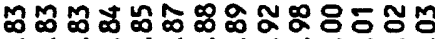

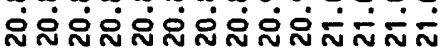
$\stackrel{n}{n}$

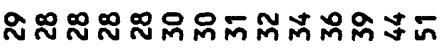

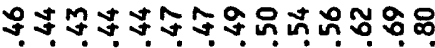

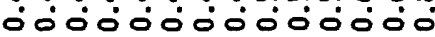

๗ั.

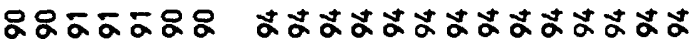

$00-0000$ $\infty \infty \infty \infty \infty \infty \infty \dot{\infty}$

$m m m m m m m m m m m m m m$

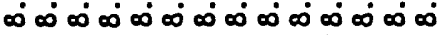

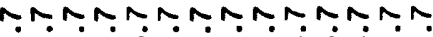
$\infty \infty \infty \infty \infty \infty \infty \infty \infty \infty \infty \infty \infty \infty \infty \infty)$

$m$

$m+0 m 0 \infty-$ $\infty \ddot{\infty} \infty \dot{\infty} \infty$

m⿻

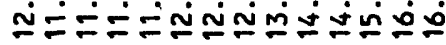

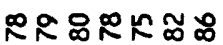

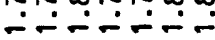

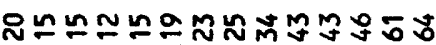

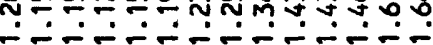

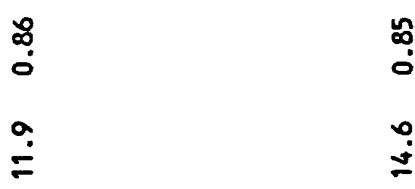

0000000

0,0000000000000

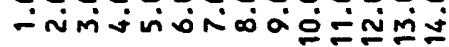

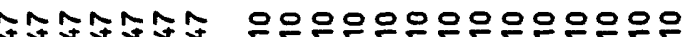

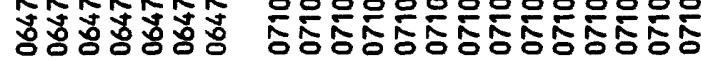

0000900

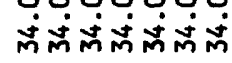

웅ㅇㅇㅇㅇㅇㅇㅇㅇㅇㅇㅇㅇㅇㅛ

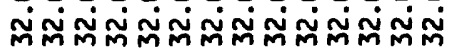




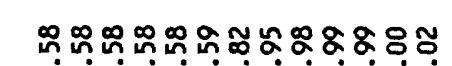

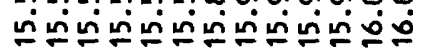

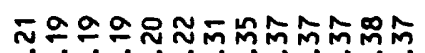

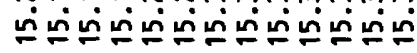

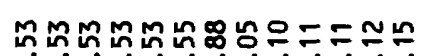

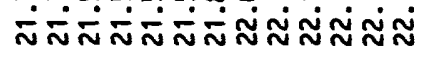
กกกกลกกลกลกล

\section{?}

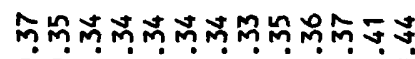
000000000000

过

芯

爻產

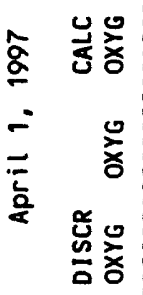

䞤돈

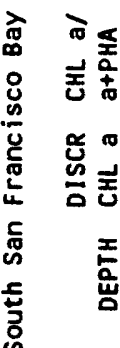

崖

恣:

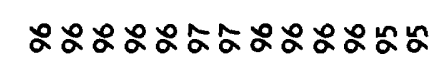

tututum untummm $\infty \infty_{\infty}^{\infty} \infty \dot{\infty} \dot{\infty} \infty \dot{\infty} \infty \dot{\infty} \infty \dot{\infty} \dot{\infty} \infty \dot{\infty} \infty \dot{\infty}$

$\infty \infty \infty \infty \infty a \infty \infty \infty \sim \sim \sim \sim$

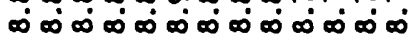

$\stackrel{+}{\infty}$

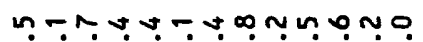

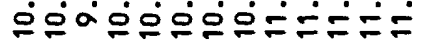

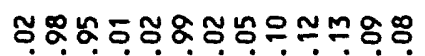

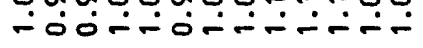

$$
\text { m. }
$$

:

m.

$\stackrel{m}{=}$

0000000000000

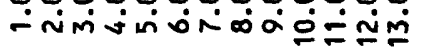

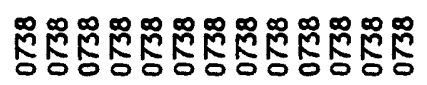

0.000000000000

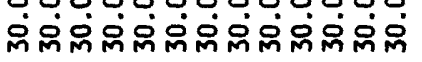

으으으으으으으ニニบบ유

ニำํํำพํำกテ 0000000000000

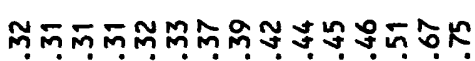

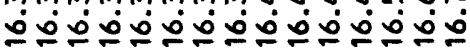

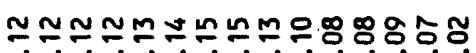

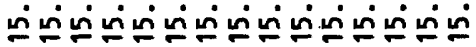

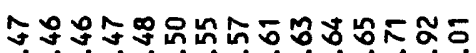

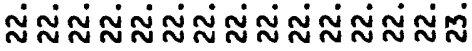

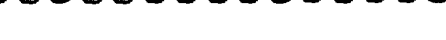

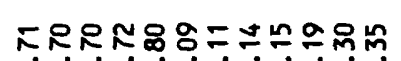

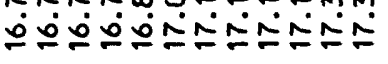

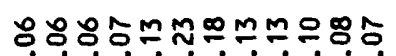

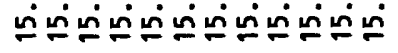

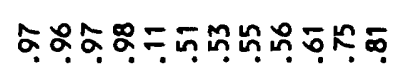

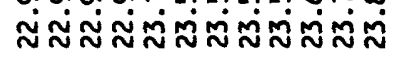

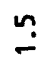

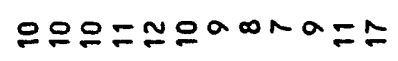

ํำำำะ์

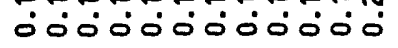
$\stackrel{\dddot{1}}{=}$

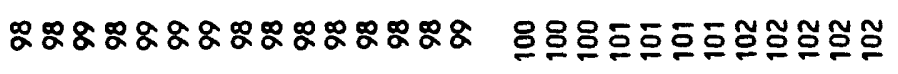

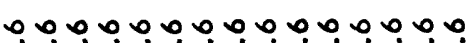

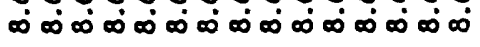

000000000000000

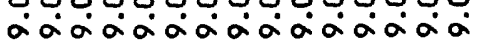

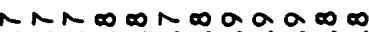
$\dot{\infty} \dot{\infty} \dot{\infty} \infty \dot{\infty} \infty \dot{\infty} \infty \dot{\infty} \infty \dot{\infty}$

$-a-a n a d a n a d$ acáaiáááa

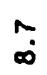

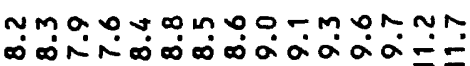

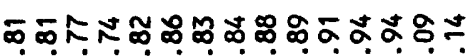

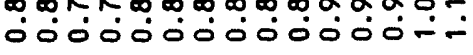

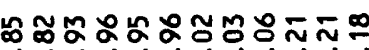

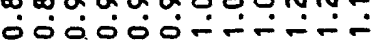

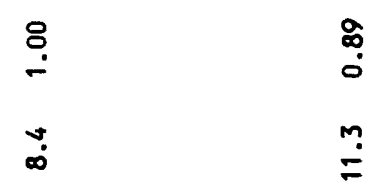

0.00000000000000 -

0.0000000000

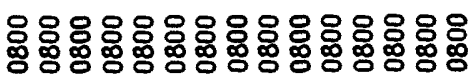

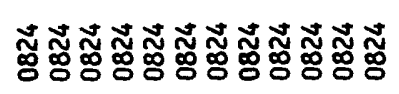

0.00 .000 .000 .0000 ล

0.000 .000 .000 .00

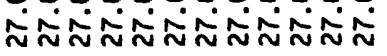




\section{ํํㅇํำำำ}

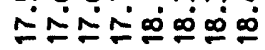

영舟욤눙ㅇ

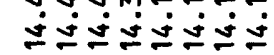

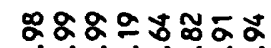

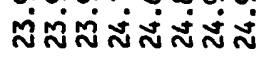

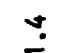

nosmora

\section{=ํํㅇํㅇำ} 0ं00000

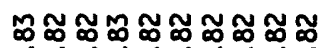

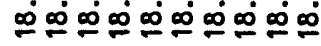

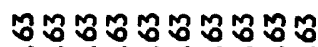

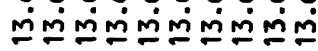

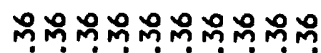

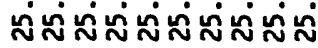

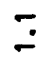

NRNDNRNDOR

$\simeq \simeq \simeq M==\simeq 00=$ 000000000 $\hat{n}$

总

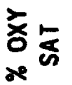

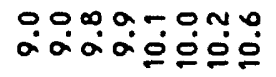

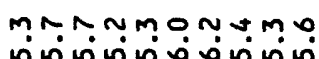

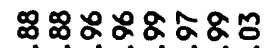
ப்0ல0்ப்-

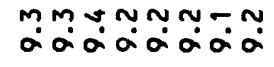

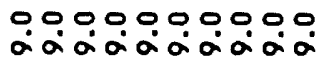
$\tilde{\infty}$

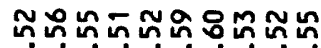
0000000000

\&

$\hat{0}$

กับ

. in

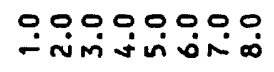

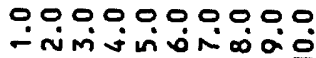

$\infty \infty a \infty a \infty \infty \infty N$ on tmmmmm

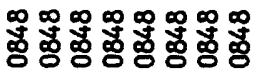

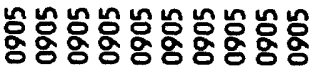

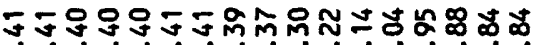

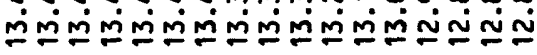

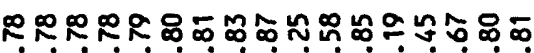

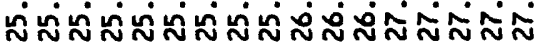

$\stackrel{0}{-}$

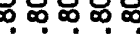

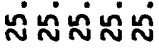

$\stackrel{0}{\circ}$

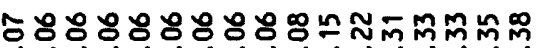
00000000000000

NะNニ= प0ं0ं

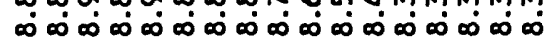

0.0000 $\infty \infty \infty \infty \infty$

\section{$\tilde{\infty}$}

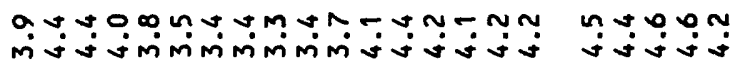

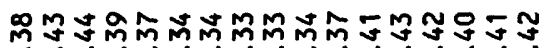

งูษษษ 000000000000000 0000

$$
\text { ā }
$$$$
\overline{5}
$$

0000000000000000000000

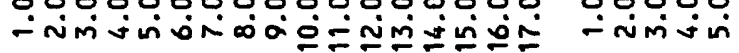

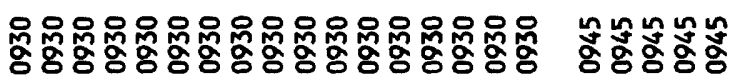

00000000000000000000000

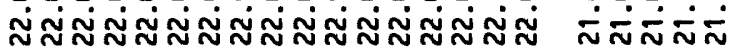




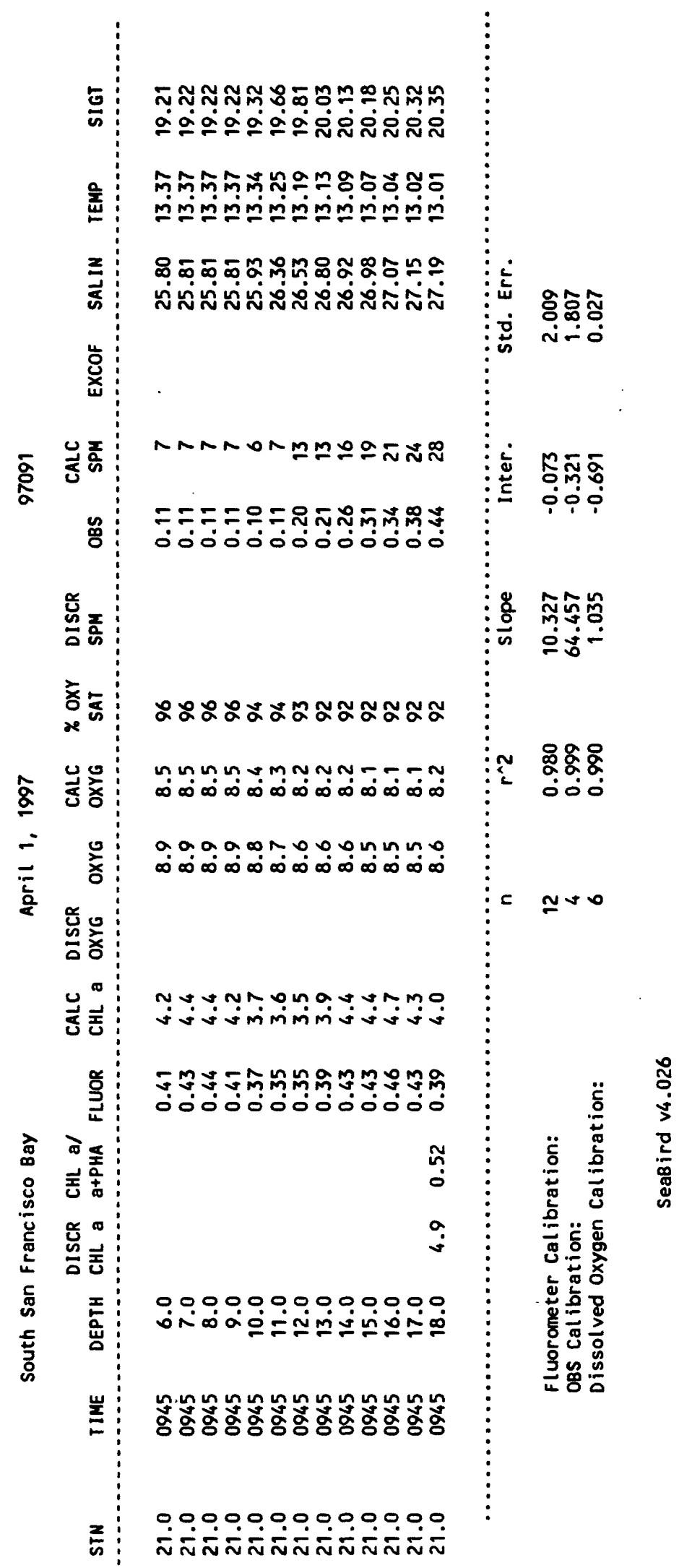




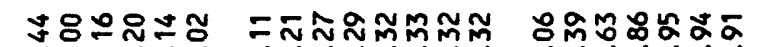

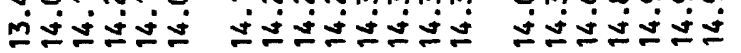

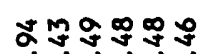

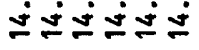

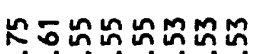

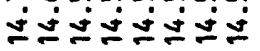

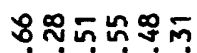

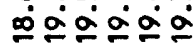

$\dot{m}$

总 巡

禺

芯空

各產

ล

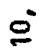

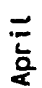

:

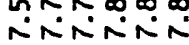

옳

웡롱

ก.m.

ที่อบำ

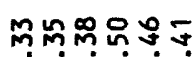

mบำ

$\stackrel{\text { กิ }}{0} \stackrel{0}{0}$

$\stackrel{\infty}{=} \quad \dot{a}$

폰

퐁

崖

z:
โ

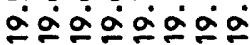

ڤ.

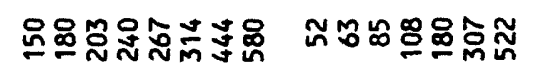

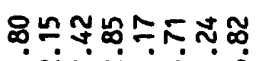

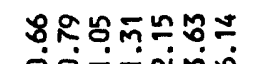

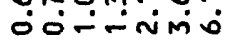

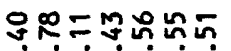

وㅇํํํํํำ

$m$

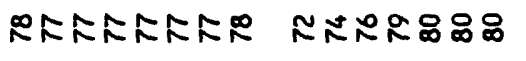

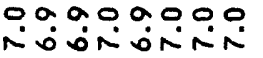

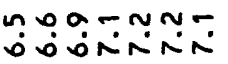

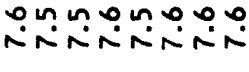

muñoñấn

momnompo gm-nmm

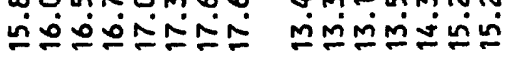
Mํํㅇำษด9

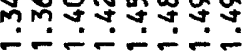

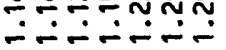

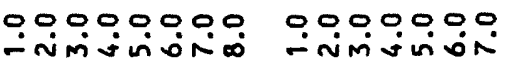

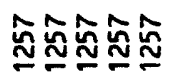

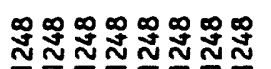

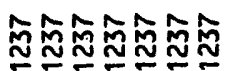

000000

00000000

0.00000

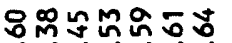

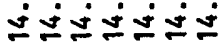

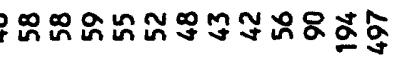

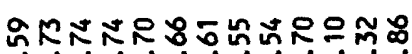

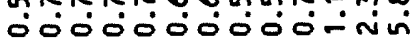

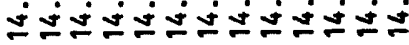

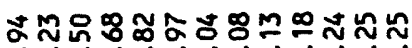

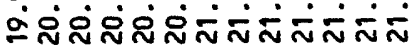

คัธกัก

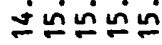

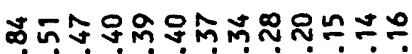

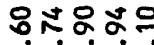

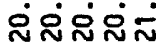

$\because$

오ำ

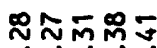
00000

$\stackrel{\infty}{\infty}$

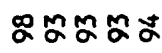

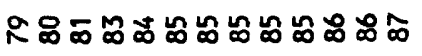

ำM?

$0 m m m=$

nnNaññanon

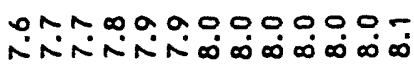

0.000 $\infty \dot{\infty} \infty \dot{\infty}$

$\stackrel{+}{\infty}$

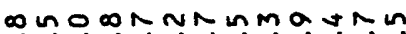

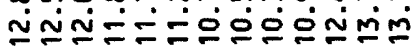

$0 \infty \times m \infty$ $=\div \div 0 \circ$

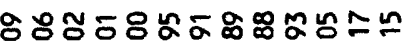
$\therefore-\because \div 00000 \div-1$

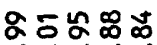

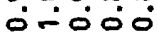

$\stackrel{n}{\circ}$

$\dot{\square}$

0000000000000

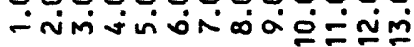

00000 - inivisin

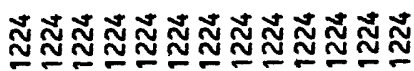

$\operatorname{nn} \backsim \backsim n$ ฐ̃ฐ̃ mंmm m
0.000

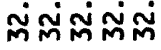




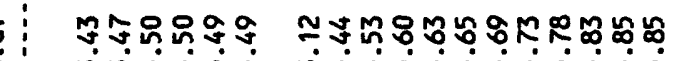

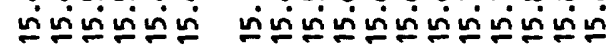

ํํํํํำำำ

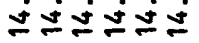

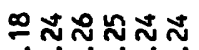

玄六市市

总

응 这

:

营

ชัญ

玉

$\therefore$

$\overline{\bar{a}}$

颉

迆突

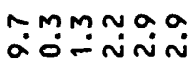

$\operatorname{lng}$

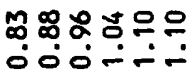

至

崖

ல숭영영

000000000000

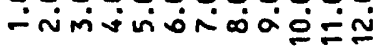

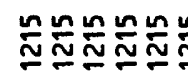

우우우웅

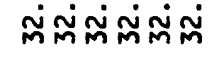

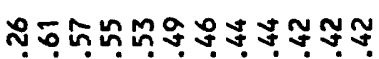

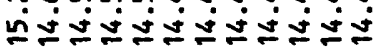

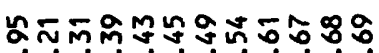

$\stackrel{i}{\sim}$

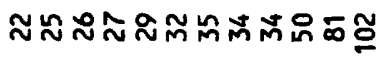

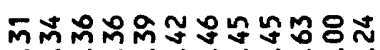

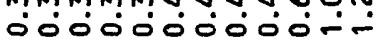

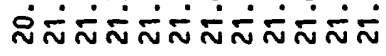

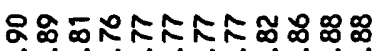

ODO000000000

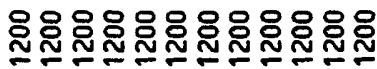

0.00000000000

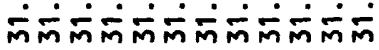

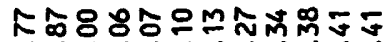

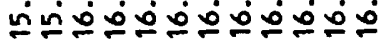

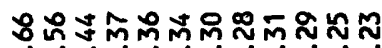

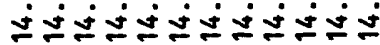

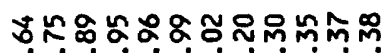

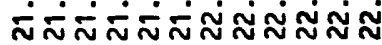

$\stackrel{\infty}{-}$

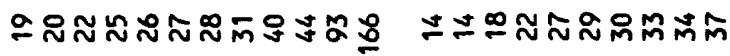

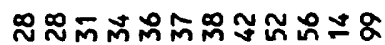
0000000000--

in

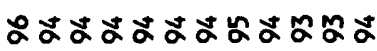

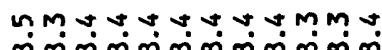

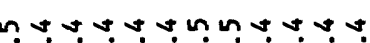
$\infty \infty_{\infty}^{\infty} \infty \infty_{\infty}^{\infty} \infty \infty_{\infty}^{\infty} \infty \infty_{\infty}^{\infty} \infty \infty_{\infty}^{\infty}$

$\tilde{\infty}$

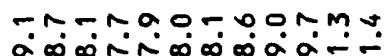

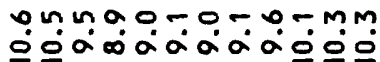

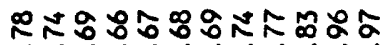
0ं0ं0000000

$\tilde{0}$

m.

$\stackrel{\infty}{\sim}$

$\stackrel{\circ}{\circ}$

000000000000

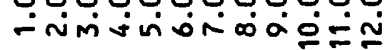

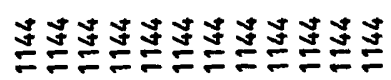

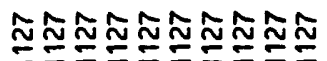

000000000000

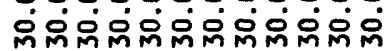

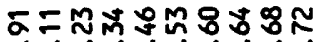

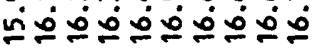

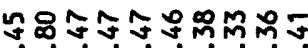

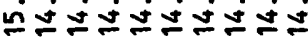

ำ $\simeq$ m

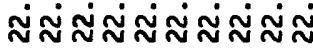

$\stackrel{0}{-}$

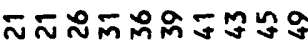
00000000

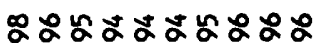

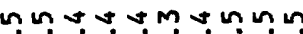
$\infty \infty \infty \dot{\infty} \infty \dot{\infty} \infty \dot{\infty} \infty \infty$

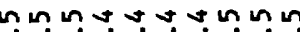

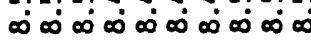

กร ก

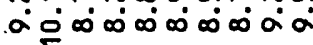

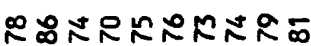

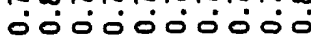

0000000000

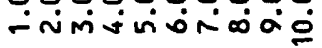




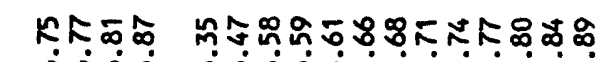

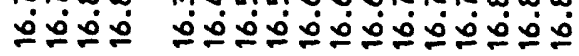

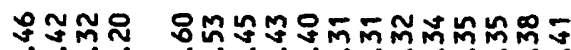

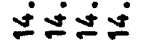

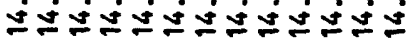

:ะำ

พก๊ก

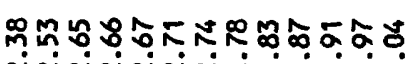

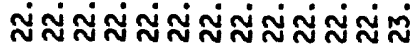

$\stackrel{\infty}{\circ}$

๕̊

爮恋

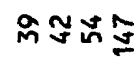

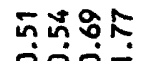

ชัญ

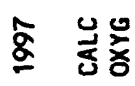

y) num

$\infty \infty \infty \infty$

苑 증

起

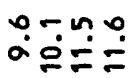

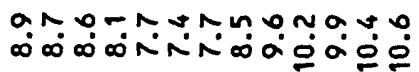

๓ $2: 8$

0.0ं

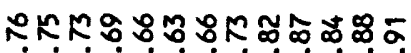

00000000000

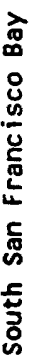

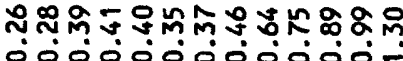

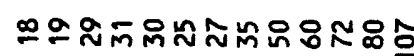

ษMmM?mm?

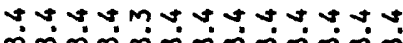
웅영

0000000000000

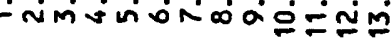

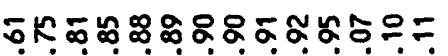

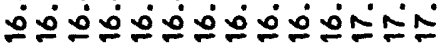

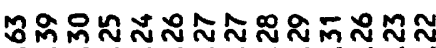

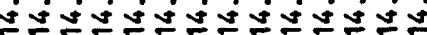

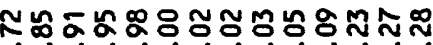

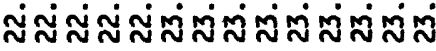

$\stackrel{\infty}{\circ}$

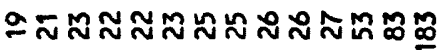

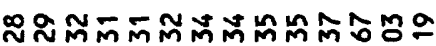

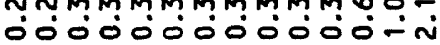

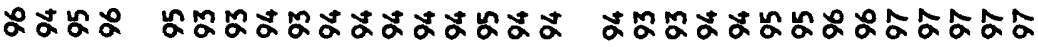

$m \sim n M+v \backsim n n 00000$ $\infty \infty_{\infty}^{\infty} \infty \infty_{\infty}^{\infty} \infty \dot{\infty} \infty \dot{\infty} \infty \dot{\infty} \infty \dot{\infty} \infty \dot{\infty}$

กnบ

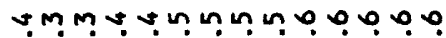
$\infty \infty \infty \infty \infty \infty \infty \infty \infty \infty \infty \infty \infty \infty \infty \infty \infty)$

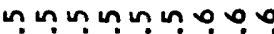
$\infty \infty \infty \infty \infty \infty \infty \infty \infty \infty$

$$
\stackrel{0}{\infty}
$$

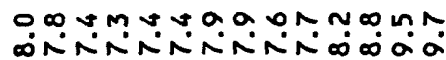

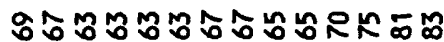

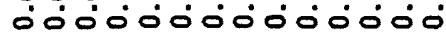

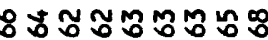
i.

$$
\text { œ̊. }
$$

$\stackrel{0}{\circ}$

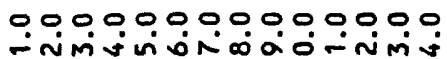

0,00000000

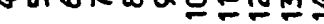

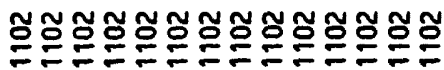

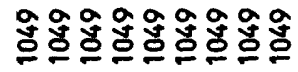

00000000000000

0.000 .0000$.

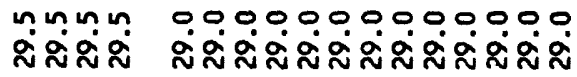

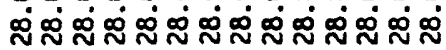


ถูณณำำำำ

으도돈

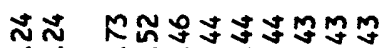

ป⿻一𠃋

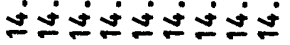

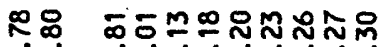

กั่

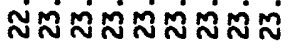

?

$\frac{8}{5}$

ษก

ニッェロッッッッล

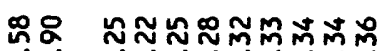

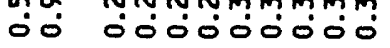

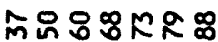

エシㅗㅗํำ

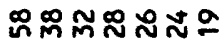

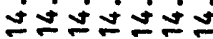

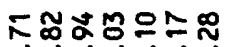

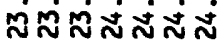

$\stackrel{+}{\square}$

$m=ㅇ ㅡ N$

テッニニ ஸ००0000

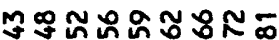

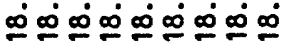

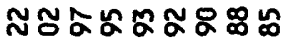

I MMMMMmm

2ธ능은ำำ

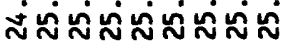

$\dddot{\Xi}$

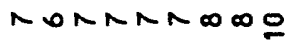

IMMMMI士ルN 00000000

$$
\stackrel{0}{a}
$$

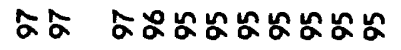

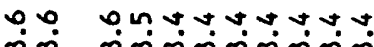

ก- 0.-

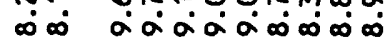

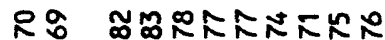
00 000000000

$\stackrel{0}{:}$

$\mathfrak{n}$

00.00000000

$\dot{0}=$ - inviviorióa

-

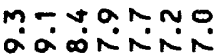

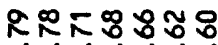
०00000

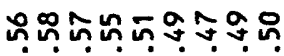
00000000

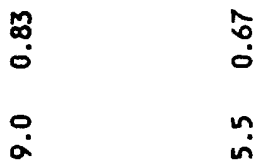

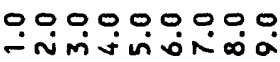

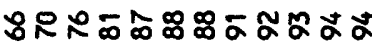

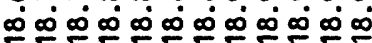

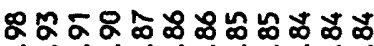
mறMmmmmmmmm

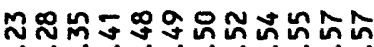

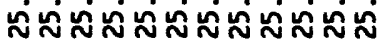
$\dddot{1}$

웅a으으는ㄷㄴ

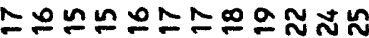
ठ0ं0்0ं0்

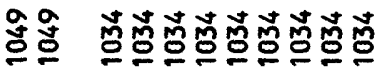

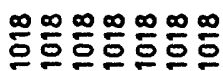

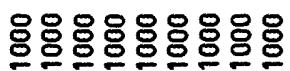

000000000000

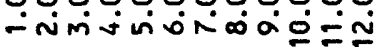

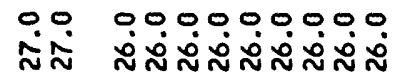

0.00000

000000000

$0,000,0000000$ 紊

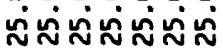

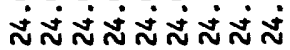

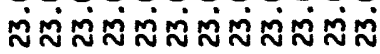




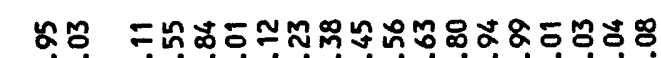

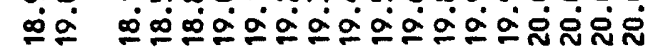

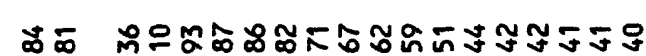

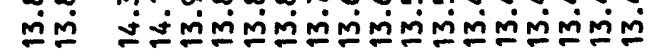

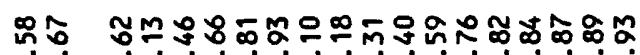

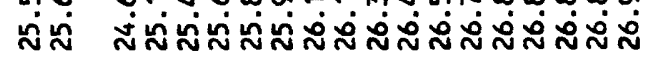
$\dddot{\Xi}$

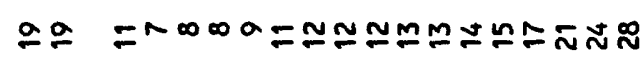

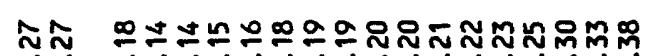
○ं 000000000000000

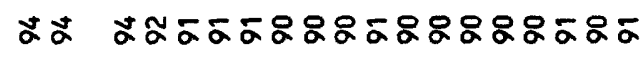

un m-0-0000000000000

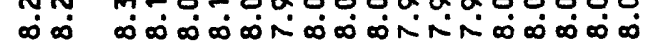

di tmann-nNn- ThNnNn

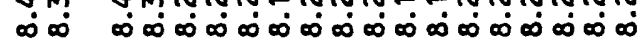

苋론

总

崖

$00 \quad 00000000000000000$

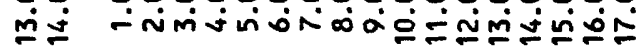

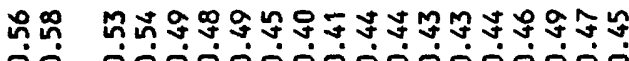
ó 00000000000000

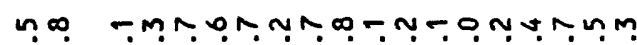

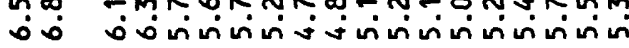

$\infty$

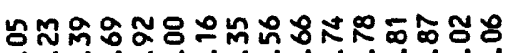

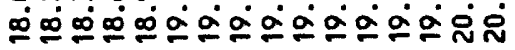

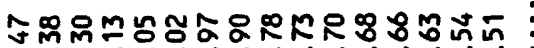
İ̃⿺廴寸

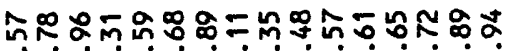

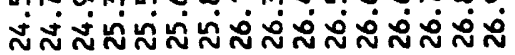
$\cong$

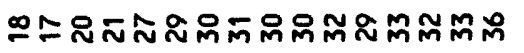

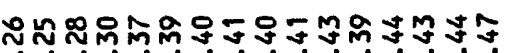
0ं000000000000

กั่

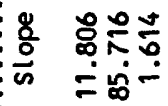

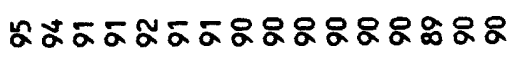

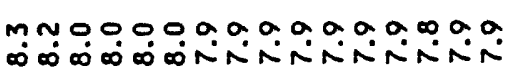

t.m

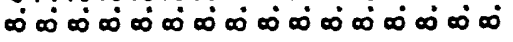

\section{$\bar{\infty}$}

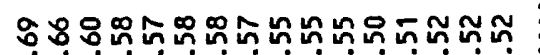
0000000000000000

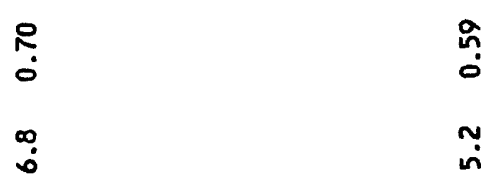

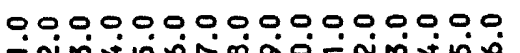

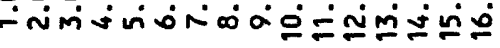

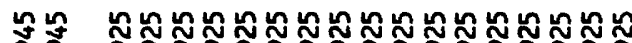
영

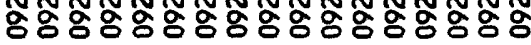

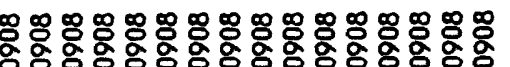

0.0000000000000000 0000000000000000000 พิ
동용 ¿ं०

$=00$

\section{ํㅗ옹 \\ inio \\ 중유ำ is n}

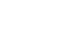

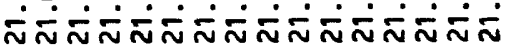


웅요웅ㅇํㅇํำ

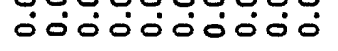

5555558.80 $\infty \phi \infty \dot{\infty} \infty \dot{\infty} \infty \dot{\infty} \infty \dot{\infty}$ 으으으으으으으으으으 00000000 ì

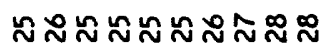

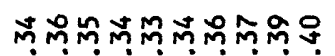
000000000 $\tilde{n}$

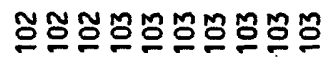

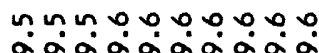

ANANANANAN

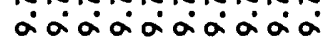

$$
\dddot{a}
$$

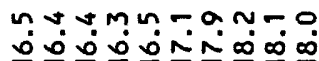

岇폰

$$
\text { gำ워ำ }
$$

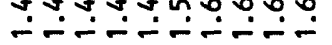

$$
\text { ọ }
$$

:

$\tilde{n}$

$$
\stackrel{\sim}{\infty}
$$

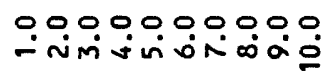

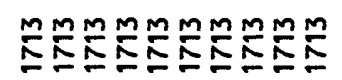

0000000000

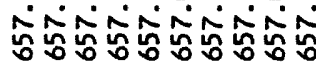

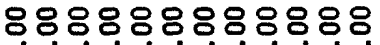
0000000000

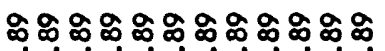

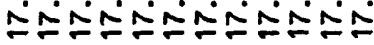

ニะ⿻上丨 ல0ं0000000

$$
\stackrel{\circ}{m}
$$

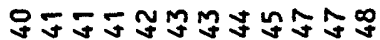

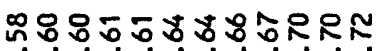
O0ं00000000

$$
\stackrel{5}{5}
$$

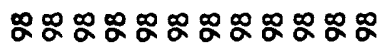

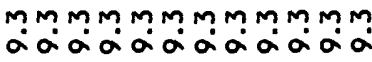

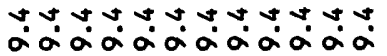

$$
\text { a }
$$

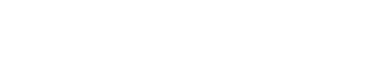

mona on mond on

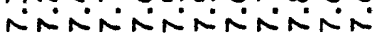

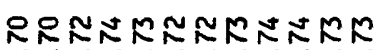
O00000000000

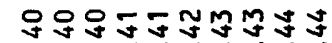
000000000

\section{TRRMRRRMRK}

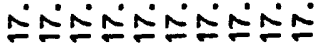

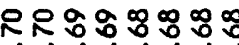

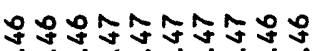
000000000

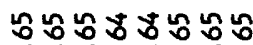

$\stackrel{m}{?}$

$\stackrel{9}{9}$

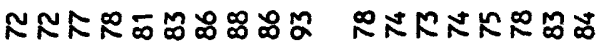

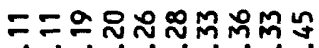

กะำำำ

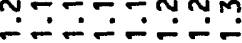

$\stackrel{\sim}{\check{\pi}}$

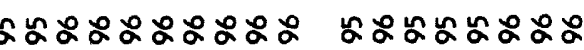

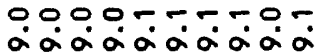

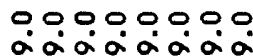

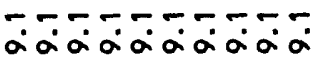

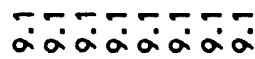
$\stackrel{\circ}{a}$

Mimago-nNn minminimitis

$\checkmark m \cup \backsim N \infty \infty N$ लिiminimimin

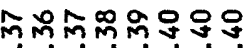
0000000

$$
\text { 号 }
$$

000000000000 - imivivion

0.00000000

0.000000 - imivinion - imisinio

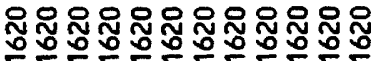

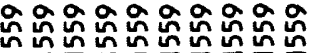

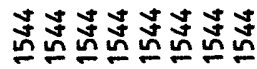

0.0000000000 gagagagajo 웅ㅇㅇㅇㅇㅇㅇㅇㅇ웅 엉우웅우웅 


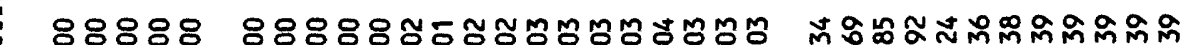

i0.00

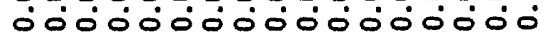

89898

ヘテヘニテ

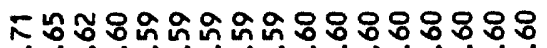

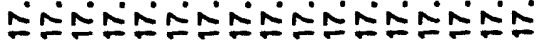

ถูกษ

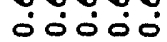

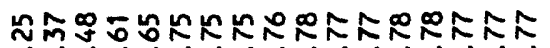

กับ

옹 농

ஐะ⿱亠凶禸ฺ

mุําmำติ

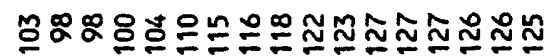

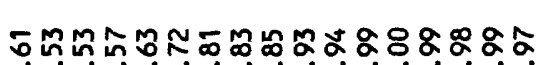

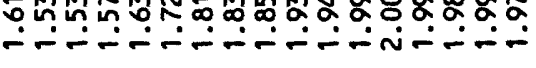

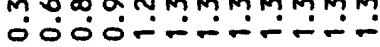

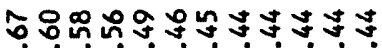

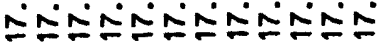

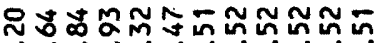
ininiminimimimim

$\stackrel{\infty}{j}$

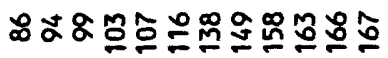

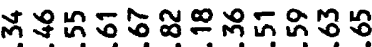

m.-

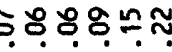

$\stackrel{\circ}{\hat{N}}$

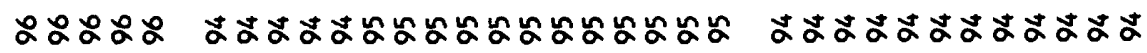

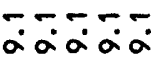

aagaagaaaa00000aa

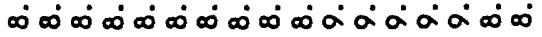

$\infty \infty \infty \infty \infty \infty \infty \infty \infty \infty \infty \infty$

$\left.\infty \infty^{0} \infty \infty \infty \infty \infty \infty \infty \infty \infty \infty \infty \infty \infty \infty\right)$

वंäa:

की

$a, \infty \infty \infty \infty \infty \infty \infty \infty \infty \infty$

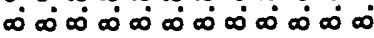

응

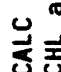

Y.M. 0.0

g.jo,

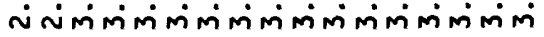

TNo.0.0000000000

Ninininimimimimim

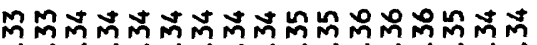

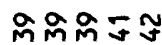

00000

o0000000000000

商.

$\stackrel{m}{m}$

옹폰

点

E

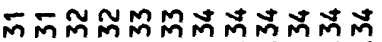

00000000000

กิกิำต̄ตm वं00ं0

î?

$\stackrel{\infty}{-}$

웅웅ํํ

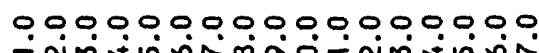

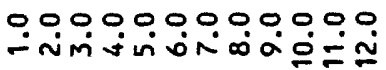

000000 으느는

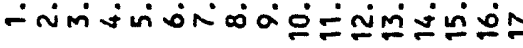

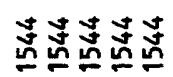

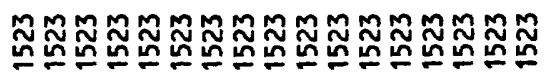

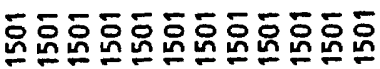

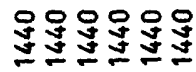

$=$

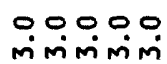

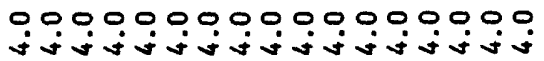

000000000000 nivinivinivinivivini

000000 000000 


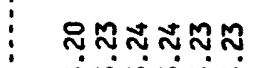
miniminimio

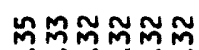

FEN

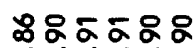

vivivivini

층 홍

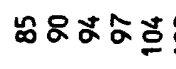

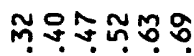

$\ddot{-ே ㅁ ㅜ ㅁ ~}$

ัㅗㅁ

ชัญ

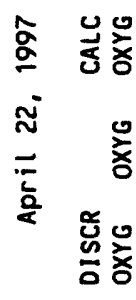

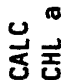

$\uparrow \cap \infty \infty \infty \infty$

inininin

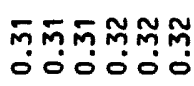

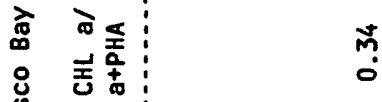

:

产

点

혼

웅영유 $\stackrel{0}{\circ}$

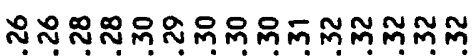

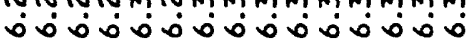

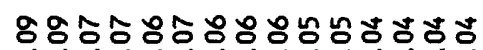

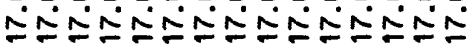

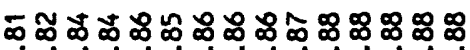

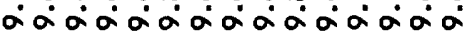

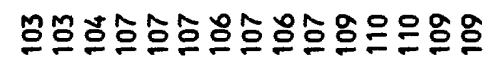

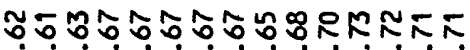

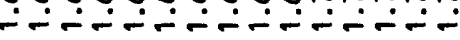

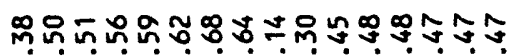

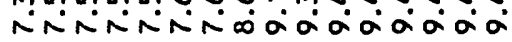

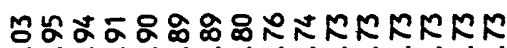

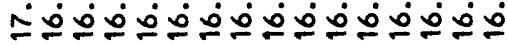

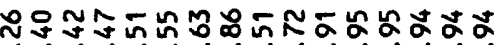

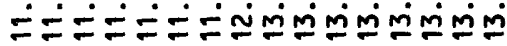
$\hat{\sim}$

ติษ

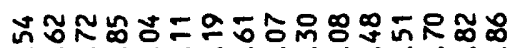
0ั00-- -
ำำ aco

๘ ஸํำ

ณ๐ั $\dot{m} \dot{m}$

$\stackrel{0}{j}$

\section{ஓి}

N $\therefore-$

$$
\ddot{n}
$$

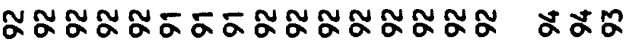

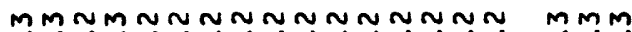

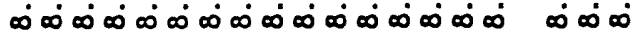

mกNกนกnนnก $\dot{\infty} \infty \dot{\infty} \infty \dot{\infty} \infty \dot{\infty} \infty \dot{\infty} \infty \dot{\infty} \infty \dot{\infty} \infty$

$\infty \infty \infty$

$$
\stackrel{n}{\infty}
$$

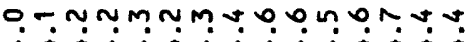

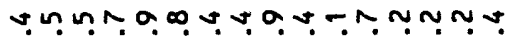

क⿺⿻一𠃋十

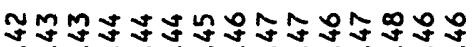

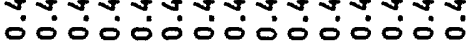

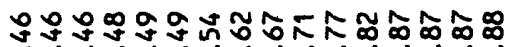

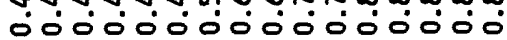

용 ๑๐

ڤึ.

$\overline{0}$

000000000000000

0000000000000000000

$\stackrel{9}{\text { ing }}$

宸

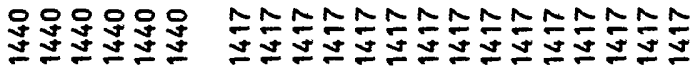

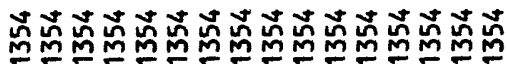

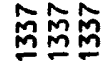

웅영영

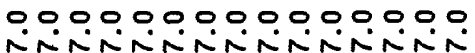

0000000000000000

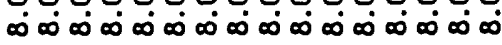

000 aंa 


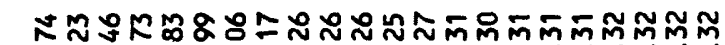

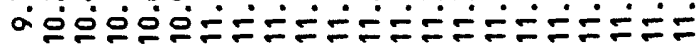

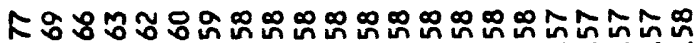

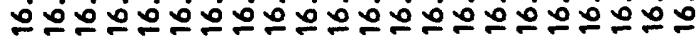

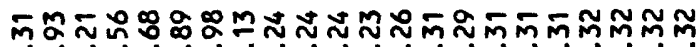

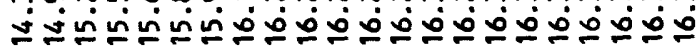

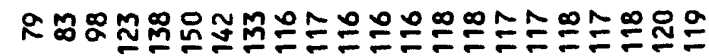

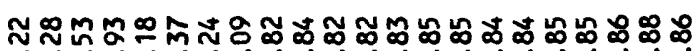
--
希产

๘

ก

$=$

总

岂范

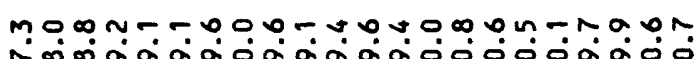

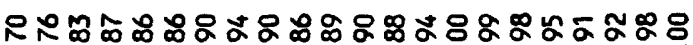

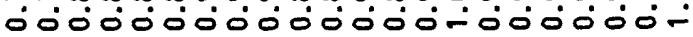

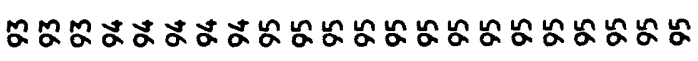

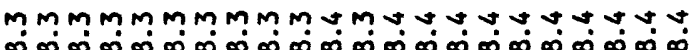
กmmmmmmmmmmmatuvtuvtut

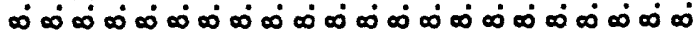

๗종 폰

웅훙

픔

崖 焉:
0000000000000000000000

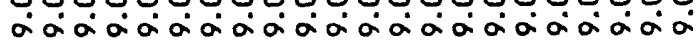

กิ

$a$

0000000000000000000000

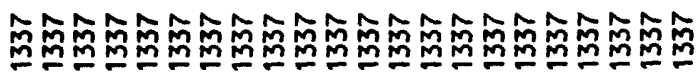

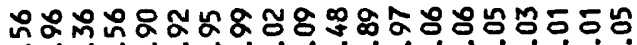

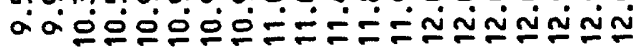

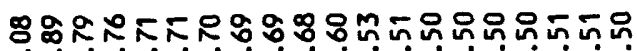

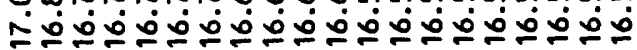

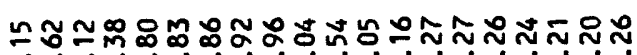

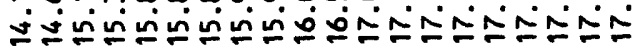
$\stackrel{0}{\circ}$

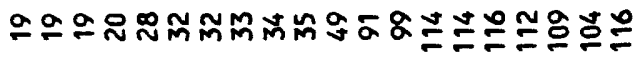

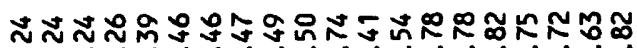

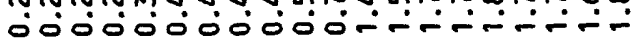

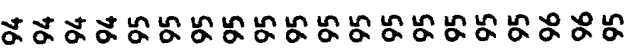

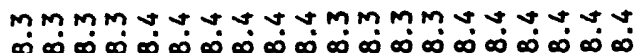

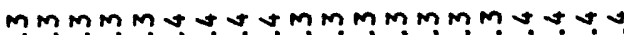

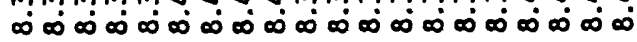

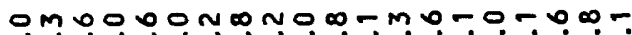

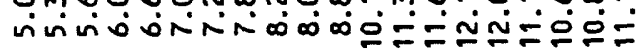

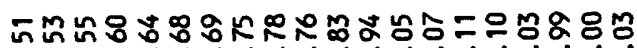

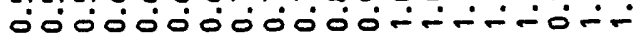

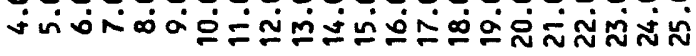

0.0000000000000000000 -

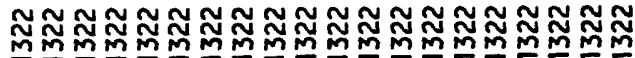

00000000000000000000

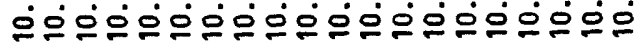




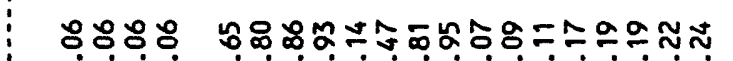

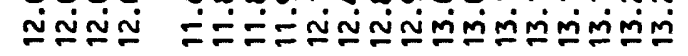

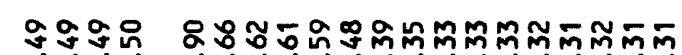

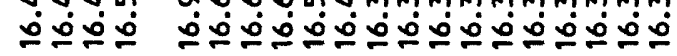

ำกำ INAS

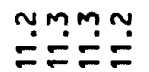

$m+m-0 \infty-m m+0 \infty+0 \infty \infty$

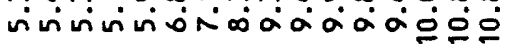

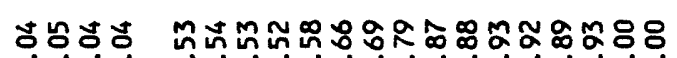
-- 000000000000

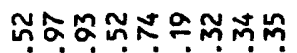

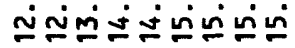

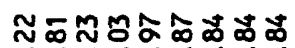
ㄷํํํำำำำ

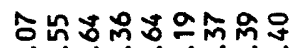

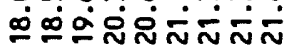

$\dddot{\sim}$

$\simeq ニ \simeq \simeq \simeq 9$ mำตำำกำกำ

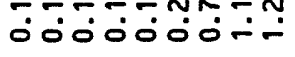
$\dddot{m}$

을옳요

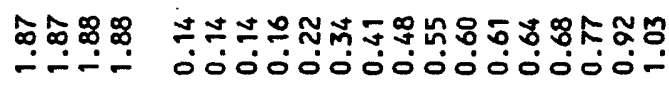

ณณณณ

\begin{abstract}
$\vec{\infty}+\vec{\infty}+\vec{\infty}+\vec{\infty}$
\end{abstract}
iे

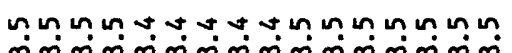

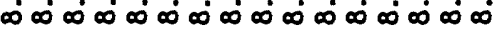

ก约约ง $\infty \infty_{\infty}^{\infty} \infty \infty_{\infty}^{\infty} \infty \infty_{\infty}^{\infty} \infty \infty_{\infty}^{\infty} \infty \infty_{\infty}^{\infty} \infty \infty_{\infty}^{\infty} \infty \infty_{\infty}^{\infty}$

$\infty$

$\ln \ln u t a t a n$ $\infty \infty_{\infty}^{\infty} \infty \dot{\infty} \infty \dot{\infty} \infty \dot{\infty}$

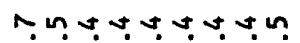
$\infty \infty_{\infty}^{\infty} \infty \infty_{\infty}^{\infty} \infty \infty_{\infty}^{\infty}$

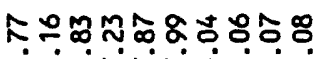

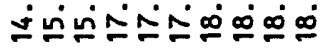

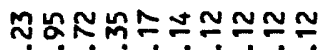

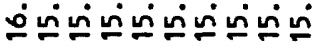

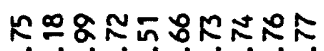

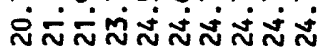

$\dddot{\square}$

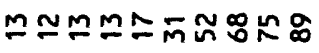

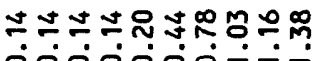

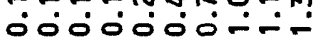

$\stackrel{9}{\circ}$

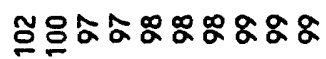

$\infty \infty \pi y t$ m n m n m n $\infty \infty_{\infty}^{\infty} \infty \omega_{\infty}^{\infty} \infty \dot{\infty} \infty \dot{\infty}$

$a \cdot \tan 4 \sin n n n$ $\infty \infty \infty \infty \infty \infty \infty \infty \infty \infty$

$\stackrel{\infty}{\infty}$

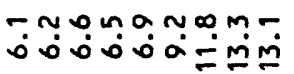

$+0.9-\infty$ m.

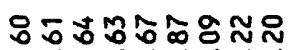
000000-ำ

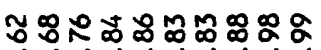
000000000

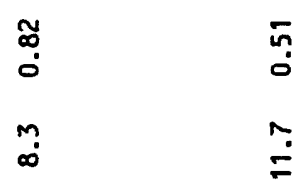

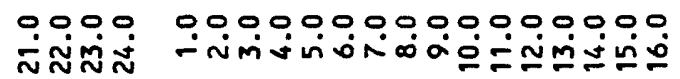

000000000

0000000000

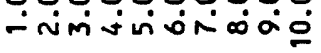

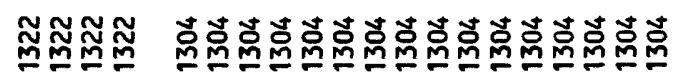

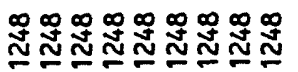

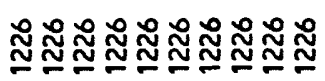

$00000 \quad 0000000000000000000$

000000000

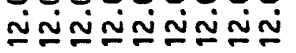

0000000000 ற்ற்ற்ற்ற்ற்ற்ற்ற் 


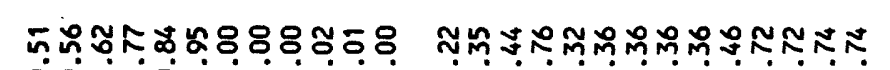

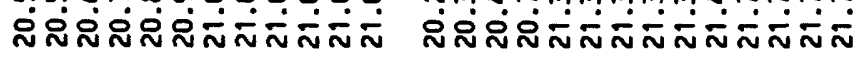

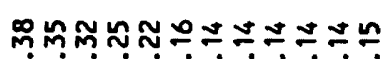

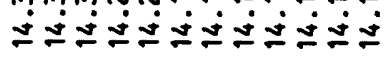

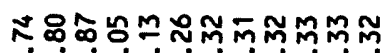

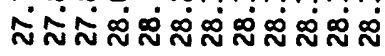

总

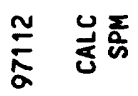

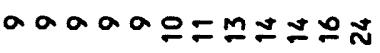

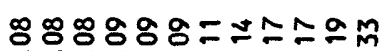
ல0000000000.

テบำ ปjป

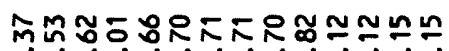

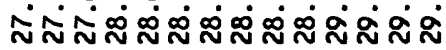
$\stackrel{9}{\circ}$

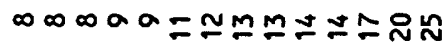

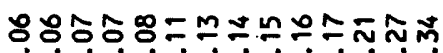
0000000000000

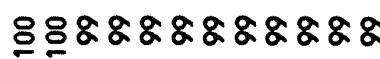

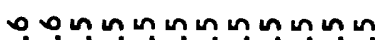
$\infty \infty^{\circ} \infty \infty^{0} \infty \infty^{\dot{\infty}} \infty \infty^{\dot{\infty}} \infty \infty^{\dot{0}}$

ọ@m

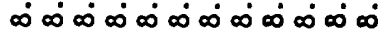
聯

远폰

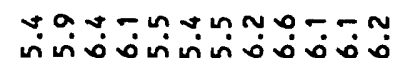
กิ

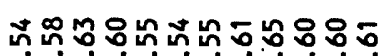
000000000000

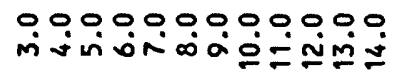

崖

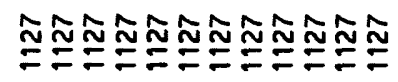

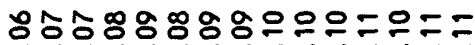

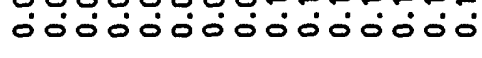

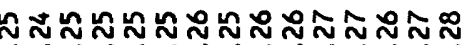

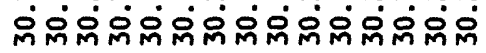

$\tilde{0}$

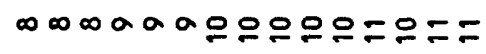
ợ

웅이웅우웅우웅우웅

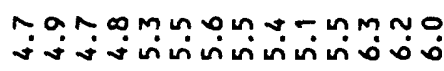

nn $v m m m a v m m m m+v$

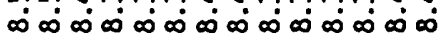

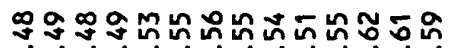
000000000000

00000000000000

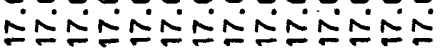

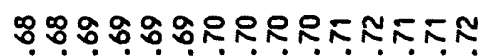

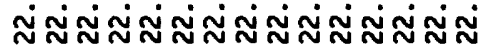

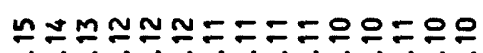

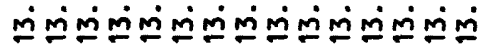

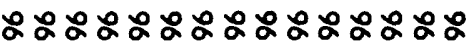
mmMmmmmmmmmm

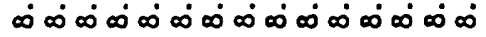

mmmmmmmmmmmmmmm

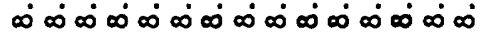

\section{$\underset{\infty}{m}$}

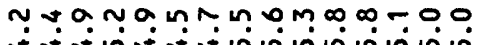

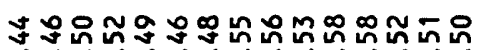

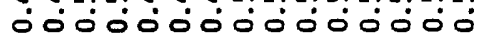
:
กั

0.000 .0000000000

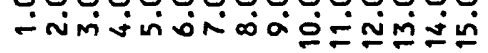

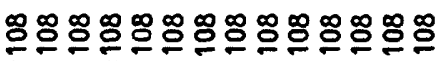

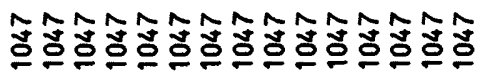

000000000000000

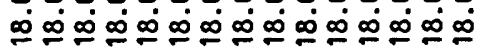




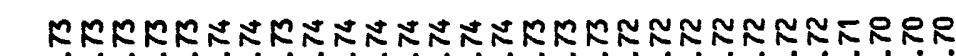

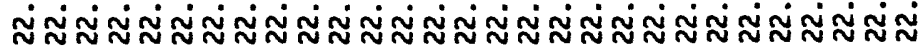

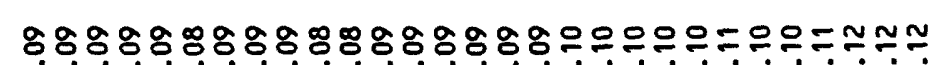

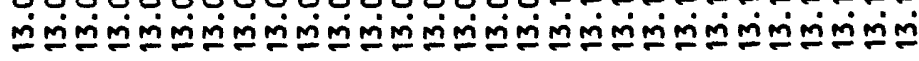

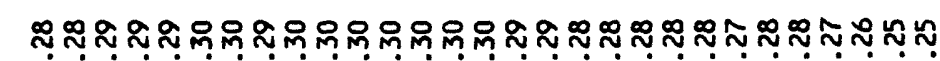

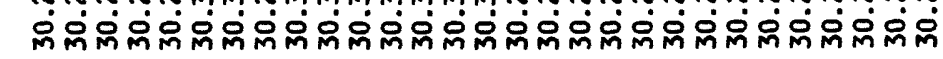

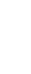

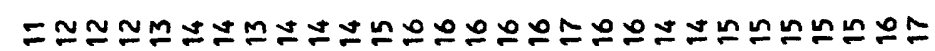

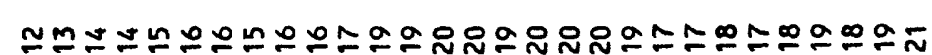
ठ0ं0ல் की

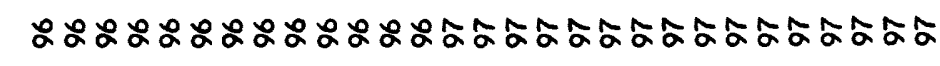

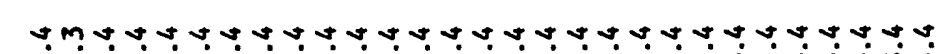

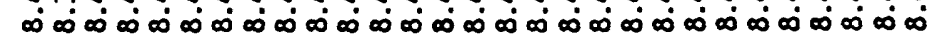

mmmmmmamatatavatavatavatatatatavatat

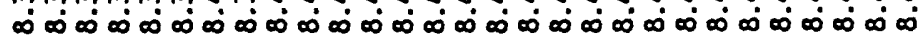

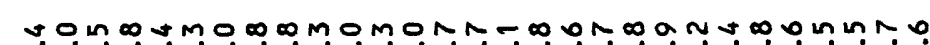
ñ.

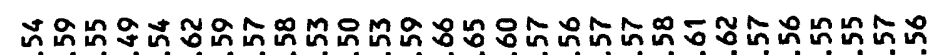

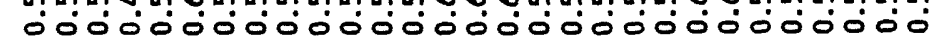
00000000000000000000000000000 


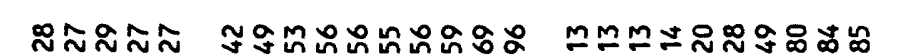

我过过

F⿻ำ

و⿱宀⿻三丨口

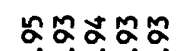

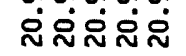

:

$\underset{\frac{1}{2}}{\cong}$

免

递

爻产

仝

$\approx$

$\overline{2}$

总

选突

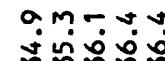

ตี้กี่ำกั

mำำษ

ininin

$$
\stackrel{N}{N} \stackrel{\infty}{0}
$$

mi

응

종

崖

00000

- invivi

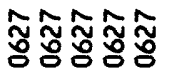

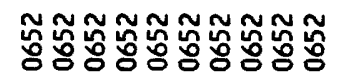

-

$\stackrel{\circ}{\circ}$

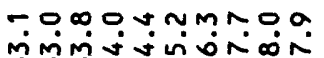

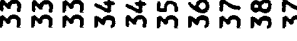

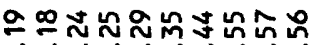
กNiNiñNiñ

落

ก.

$\stackrel{\sim}{\sim}$

$\stackrel{n}{\sim}$

0000000000

- imivioñó

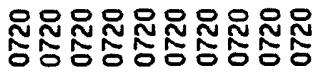

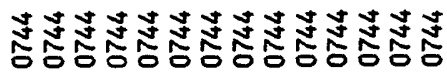

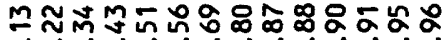

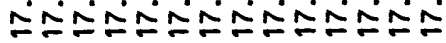

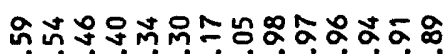

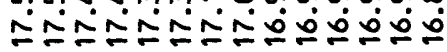

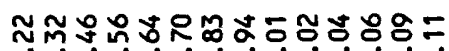

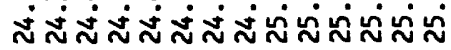

$\stackrel{\infty}{-}$

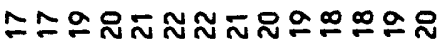

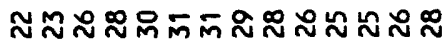
ठ00000000000

$\stackrel{\circ}{\infty}$

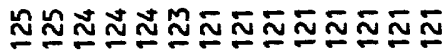

mMnnก-

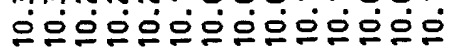

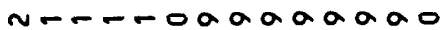

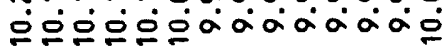

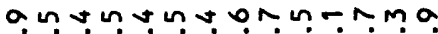

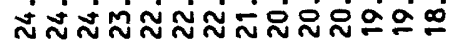

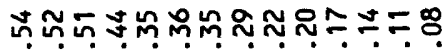

00000000000000

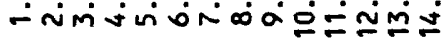

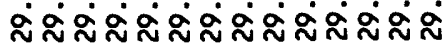




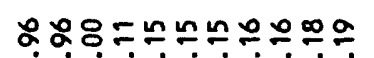

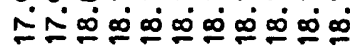

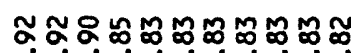

ㅇํํํำำ

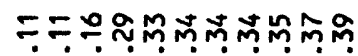

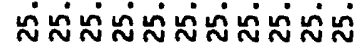

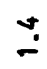

종

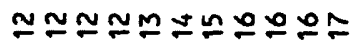

느느ํำกำก

0000000ல்0

䔳

爻衷

ڤ્

กี

항

웛

迅퐁

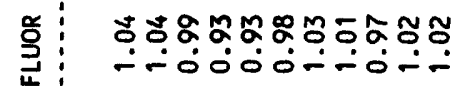

.

$\dot{0}$

$\stackrel{\circ}{\sim}$

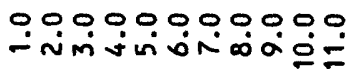

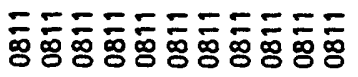

0.0000000000

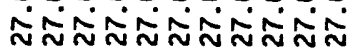

눙ํํ우웅ㅇㅇㅇ

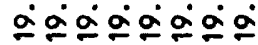

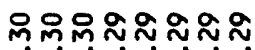

ㅇํㅇํํㅇำ

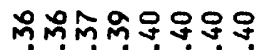

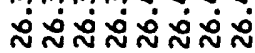

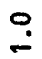

$\infty \infty \wedge a= \pm m$

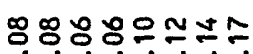
व0.000000
叫

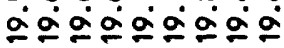

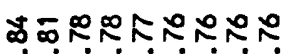

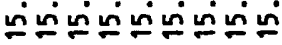

สูㄴํㅇㄴㅇํㅇำำ

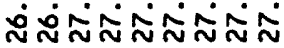

$\stackrel{\infty}{\circ}$

num uno mo

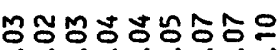
ऽ0000000

$\dddot{1}$

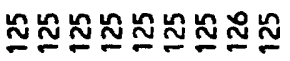

นn un un un un un un

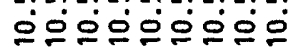

Tmmmmmut

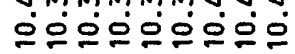

$\stackrel{\circ}{\circ}$

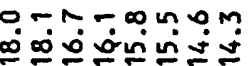

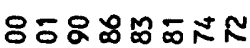
-O0.00000

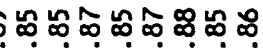
00000000

: 10

$\stackrel{m}{5}$

$\hat{\infty}$

0.0000000 -nimisinio

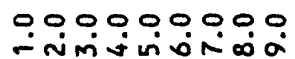

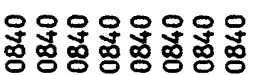

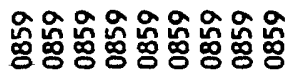

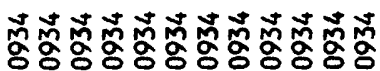

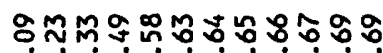

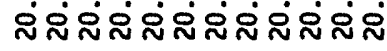

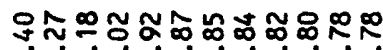

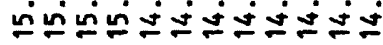

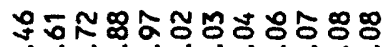
กลลกล ก.

$\tan \tan 0000=$ N

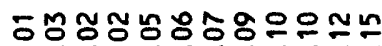
000000000000

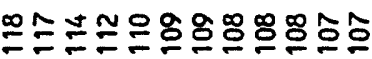

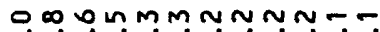
iajarajááa

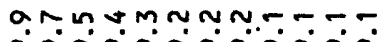
ajááajáa

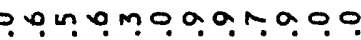

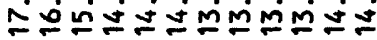

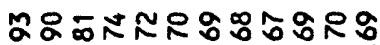
هं०0ं0்

0.00000000000

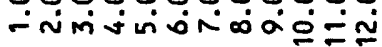

0,0000000 0.00000000 0.00000000000

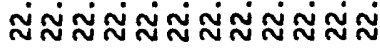




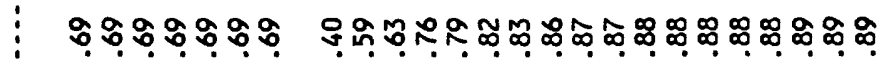

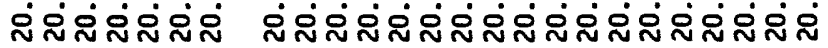

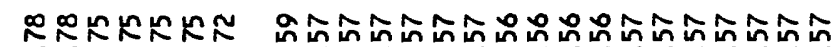

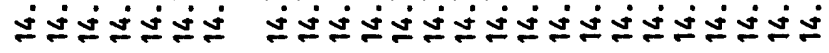

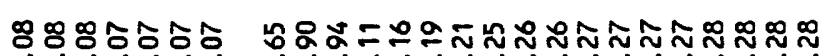

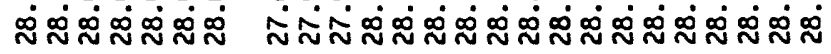
$\stackrel{a}{\circ}$

ปะกีลกำ

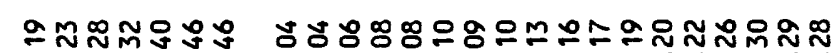

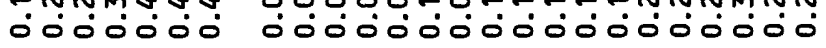
m.

ชิำำ نं०

三命高 inimi

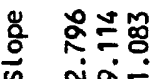
各率

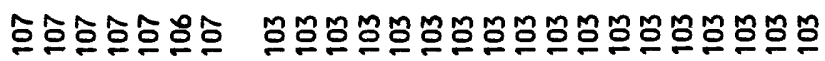

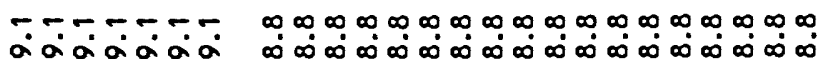

$\infty \sim \infty \infty \infty \infty \infty \infty \infty \infty \infty \infty \infty \infty \infty \infty \infty \infty$

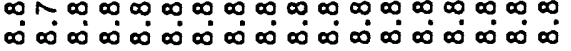
$\stackrel{\infty}{\infty}$

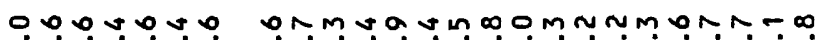

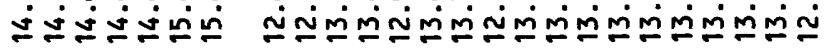

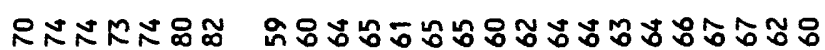

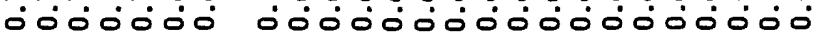

๕

0000000000000000000000000 mín

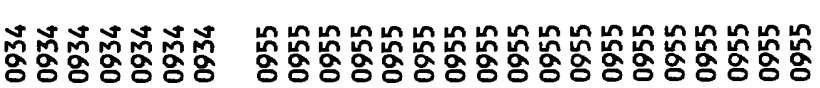

00000000000000000000000000

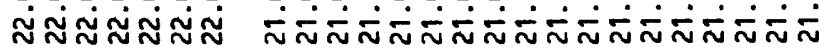


ที่

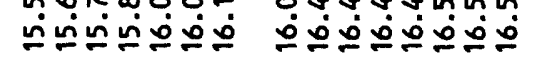

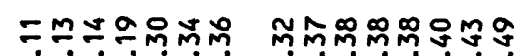

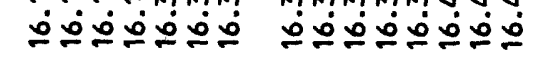

กะฒฺำน

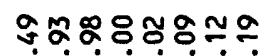

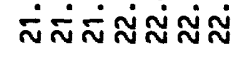

m

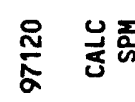

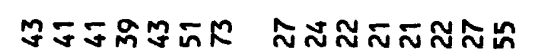

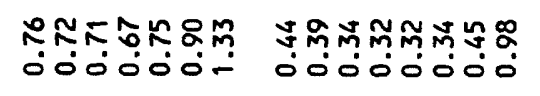

ós

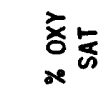

宜 过

음

:

징

总

뚫

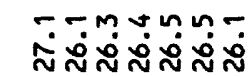

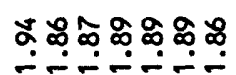

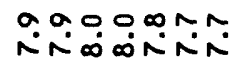

-

$\stackrel{\infty}{i}$

$\hat{n}$

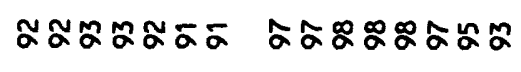

$m m m+409$

$\infty \infty \infty \infty \infty \infty \infty$

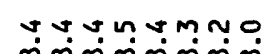

$\infty \infty_{\infty}^{\infty} \infty \infty_{\infty}^{\infty} \infty \infty_{\infty}^{\infty}$

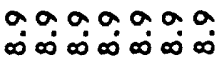

$\infty 0.000 .00$

$\infty \infty_{\infty}^{\infty} \dot{\infty} \infty \dot{\infty} \dot{\infty} \dot{\infty}$

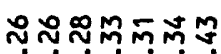

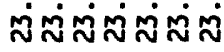

ì

ลักตับษับิ์

ง Mูกำกำ

000000-

옹ํํํํㅇํำ

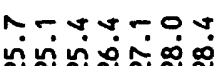

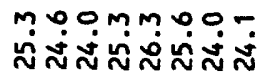

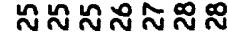

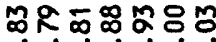

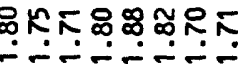

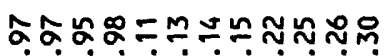

은도도돈

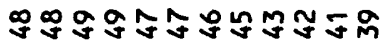

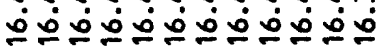

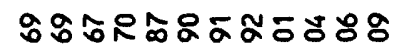

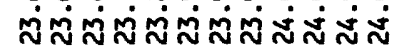

$\stackrel{\infty}{-}$

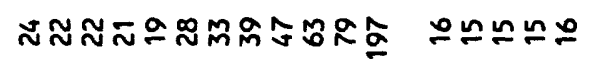

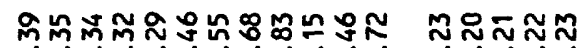

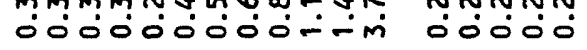

$\stackrel{ }{\stackrel{1}{5}}$

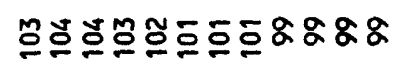

NNDroponntmmm?

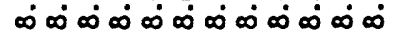

Tonn 0

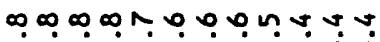

$\infty$

$\infty \infty \infty \infty \infty \infty \infty \infty \infty \infty \infty \infty \infty)$

$\tilde{\infty}$

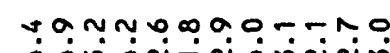

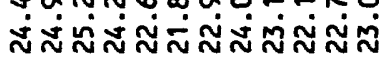

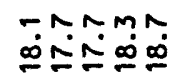

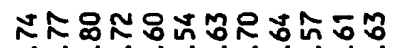

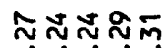

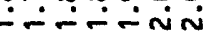

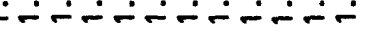

ำำ-

$\begin{array}{ll}n & n \\ 0 & 0\end{array}$

ڤ̊.

ஸ்

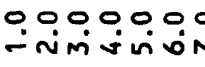

0.0000000

0000000

$\infty$

$\stackrel{\square}{\circ}$

$\stackrel{\infty}{\sim}$

崖

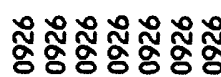

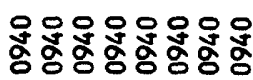

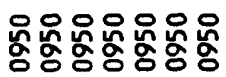

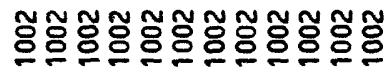

ำํำำำ

0000000

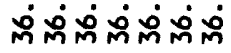

00000000

0000000

000000000000

00000

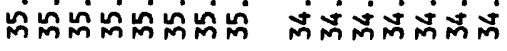

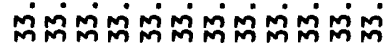

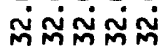




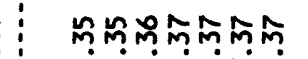
ニㅗㅗ

ษฐซซำ ํํํํํํำ

⿻ㅡ으으응

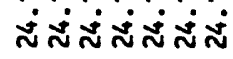

$\stackrel{2}{\mathfrak{\vdots}}$

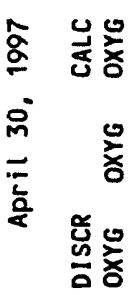

돓롱

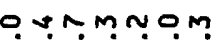

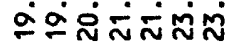
두두

$\stackrel{\infty}{\leftrightarrow}$

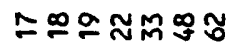

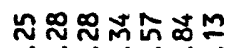
000000

유융요영

חִ

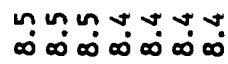

ì

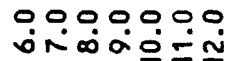

0000000 ผี่

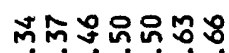

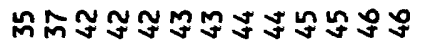

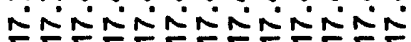

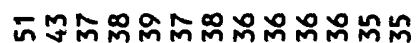

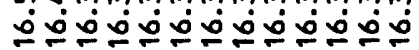

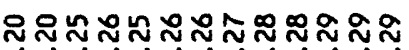

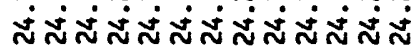

$\stackrel{\circ}{-}$

$\infty$

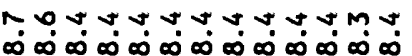

Anvmmmmmmmm nm

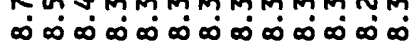

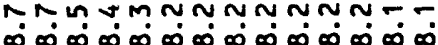

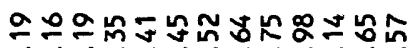
000000000-n

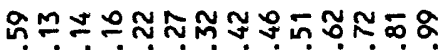
0000000000000

$\stackrel{\dddot{1}}{=}$

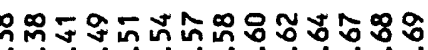

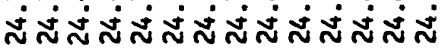

$\dddot{-}$

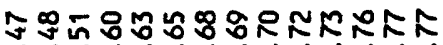

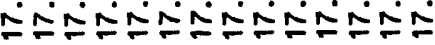

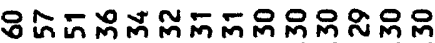

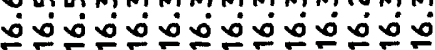

นก๊ำำ ำ อํํํㅇำ

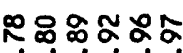

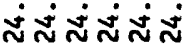

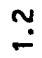

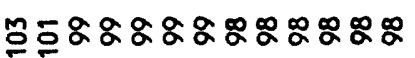

๖ั

ํㅜㅇㅎㅗ

$\infty \infty \infty \infty \infty \infty \infty \infty \infty \infty \infty \infty)$

$\operatorname{sinmnN-}$ $\infty \infty_{\infty}^{\infty} \infty \infty_{\infty}^{\infty} \infty$

$\infty$ NOUJUMmMMMMNN

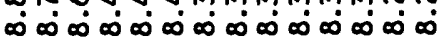

$\operatorname{nntmmn}$ $\infty \infty \infty \infty \infty \infty \infty$

$\stackrel{\infty}{\infty}$

0 -

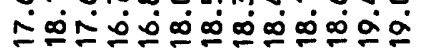

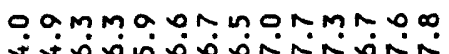

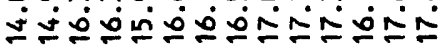

-0.00\%

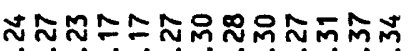

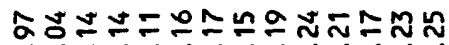

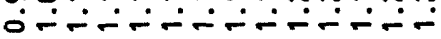

ฆธะัณณ

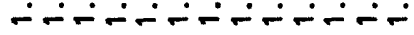

ஜ̊.

:ํ.

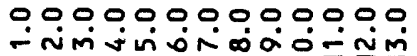

ง

$\stackrel{m}{m}$

ஸ்

00000000000000 -

000000 - invinio

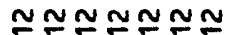

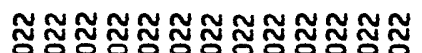

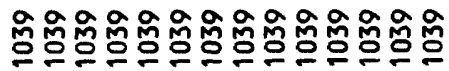

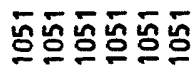

0.00000000000

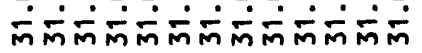

00000000000000

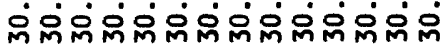

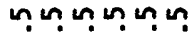

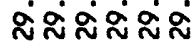


ธธณณณปัชัป $\stackrel{\infty}{\infty} \infty \infty \infty \infty$

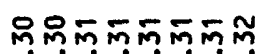
ㅇํㅇㅇㅇ

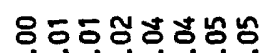

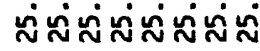

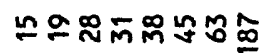

กิ๊ क0000ं

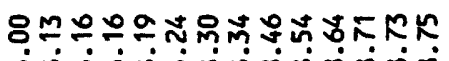

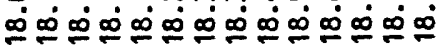

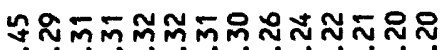

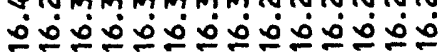

ำำํำ

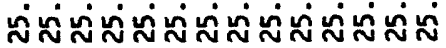

$\Xi$

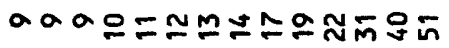

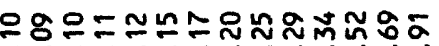

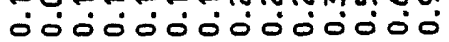

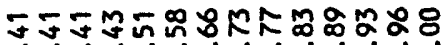

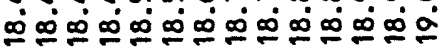

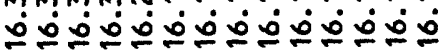

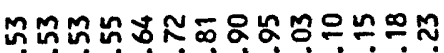

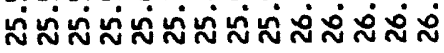

$\stackrel{9}{-}$

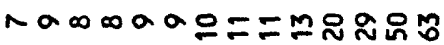

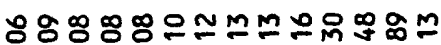

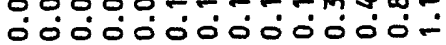

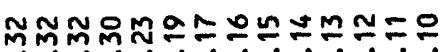

ถำนnนก้น

$\infty \nsubseteq \stackrel{\infty}{\circ}$

的的界量 $\div \div \div 0$ NRKR ล่งก

$\stackrel{9}{-}$

$\infty \infty \infty \infty$

동ํㅇㅇㅛ 0ं0்

$\hat{i}$

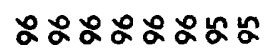

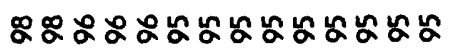

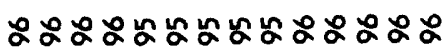

용ㅇㅇㅇ

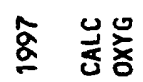

$F=\div \div 000$.

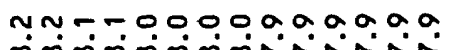

-

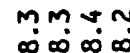

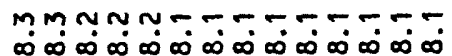

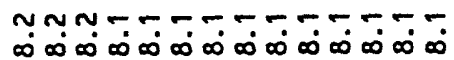

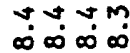

סَ:

$\stackrel{+}{\infty}$

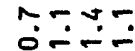

Q

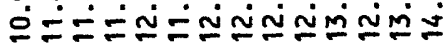

ำำ

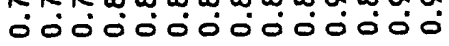

순ำ ¿0ं0

$\stackrel{5}{0}$

a

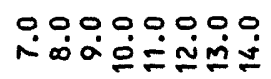

00000000000000

0.0000000000000

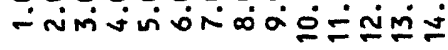

0000

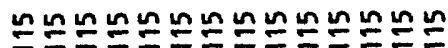

욣요

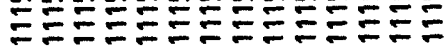

0.000 00000000000000

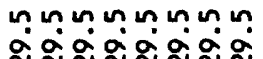
$0.000,0,0,0,0,0,0,0$

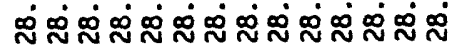




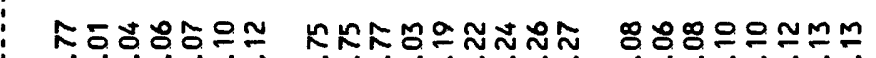

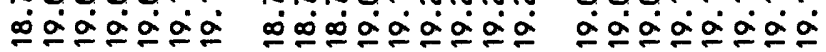

สะสมสมสะส

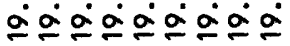

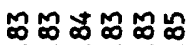

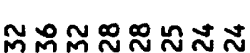

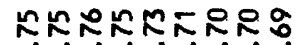

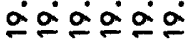

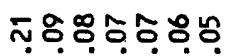

ㅇำำㅇํํํำ

ㅇํㅇํㅇํㅇํㅇ

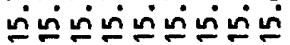

융ㅇㅇㅇㅛ 요ェํำ

ํํํํํำำำ

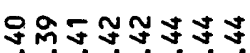

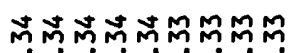

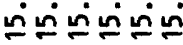

누ำกำกำ

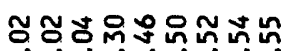

क०००00000

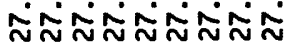

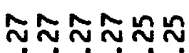

กักำกำ

o.

$\stackrel{0}{-}$

a.

ลก̃กลกล

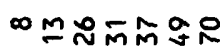

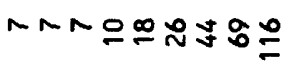

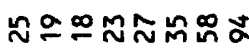

$00000 \simeq \pm N N$

$\stackrel{\infty}{0}$

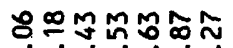

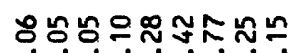

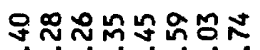

영요ニッㄴ

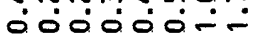

वं000000

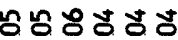
-000000-ก

$m_{\infty}^{m}$

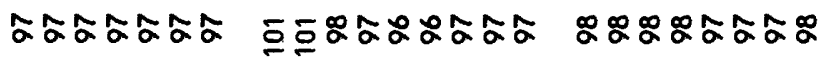

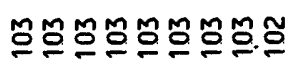

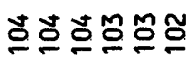

ล

के

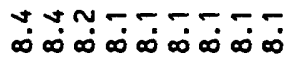

กับ

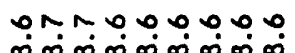

NヘNー00

บ $M m m m$

กำ $m$ N $N$ N

$\infty \infty_{\infty}^{\infty} \infty \infty_{\infty}^{\infty} \infty \infty^{\infty}$

$\infty \infty_{\infty}^{\infty} \infty \infty_{\infty}^{\infty} \infty \infty_{\infty}^{\infty} \infty \omega^{\infty}$

MMMMNNNM

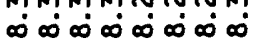

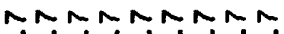

$\infty \infty \infty \infty \infty \infty$

总

范范

M $\infty \sim \infty 0$

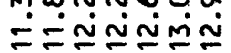

กำ

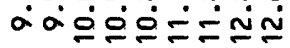

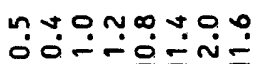

$\infty_{\infty}^{\infty} \infty \infty_{\infty}^{\infty} \infty \infty_{\infty}^{\infty} \infty \infty_{\infty}$

ヘヘヘヘヘ。

$\stackrel{\infty}{\infty}$

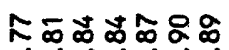

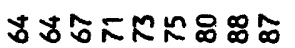

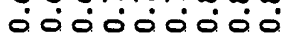

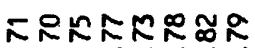

व0000000

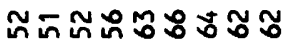

0ं0 0 0ं

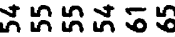

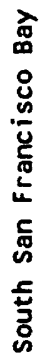

?

๓

๕ั

$\infty$

$\hat{a}$

递 몽

$\doteqdot$

$\infty$

00000

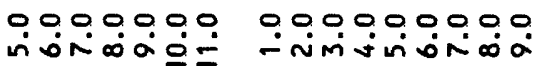

00000000

000000000000000

- imivinis

-nivionio

- imingo:

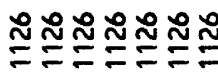

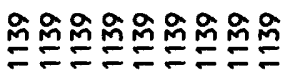

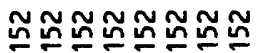

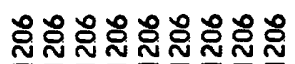

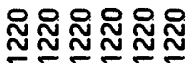

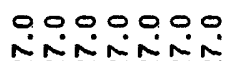

0.0000000

0.000000

0.0 .000 .000

000000

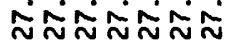

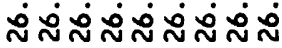

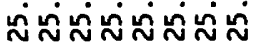

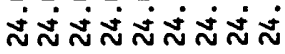

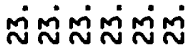




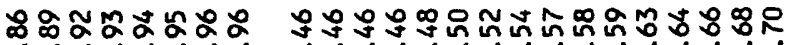

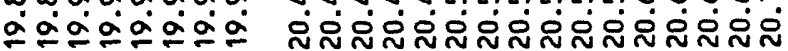

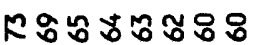

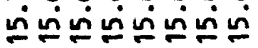

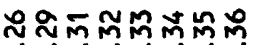
ลักลกลกลกลก<smiles>[Mg][Mg]</smiles>

$\hat{0}$

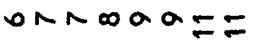

농ํㅇㅇㅇ으는

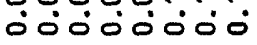

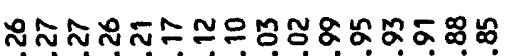

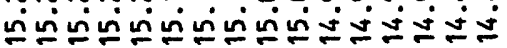

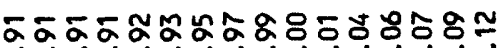

$$
\dot{0}
$$

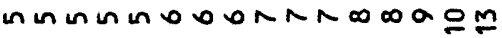

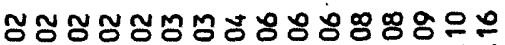

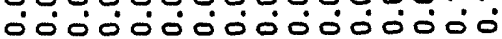

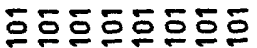

$\ln \min \min n \min$ $\infty \infty \infty \infty \infty \infty \infty \infty \dot{\infty} \infty \dot{\infty}$

00000000 $\infty \infty \infty \infty \infty \infty \infty \infty \infty \infty \infty$

ชิ 妾

눈

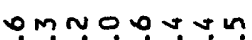
$\alpha \dot{\alpha} \alpha \dot{\alpha} \infty \dot{\infty} \infty \dot{\infty} \infty$

m Noninsamonamagn

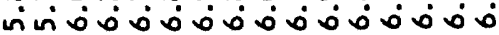

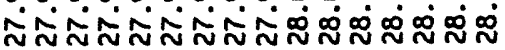

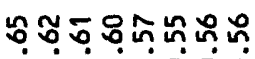
0000000

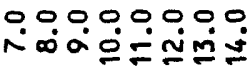

0.00000000000000

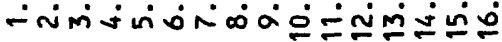

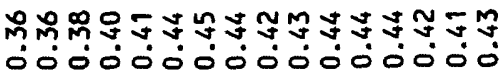

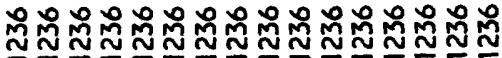

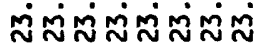

0000000000000000

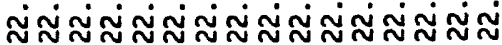

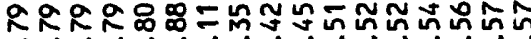

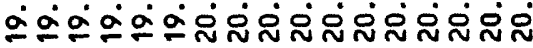

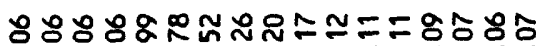

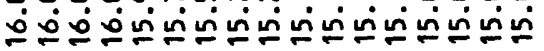

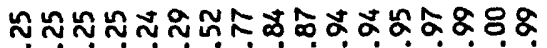

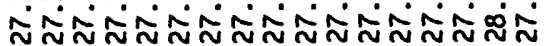

$\hat{0}$

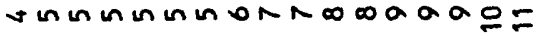

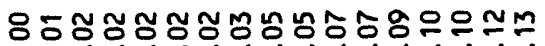

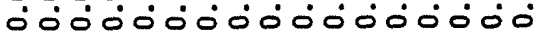

$\stackrel{\infty}{m}$

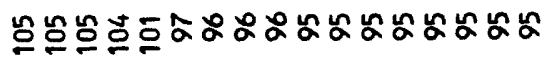

NNon? no-

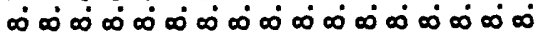

$\infty \infty \infty \sim m M N \sim \sim \sim N \sim \sim-7-N$

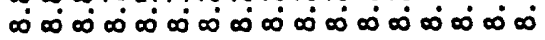

$\stackrel{\circ}{\infty}$

-munoummomm

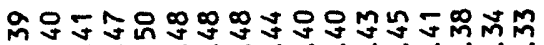
000000000000000

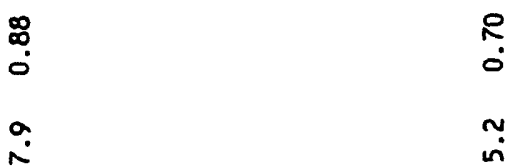

0000000000000000000

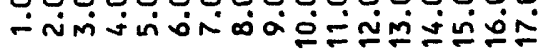

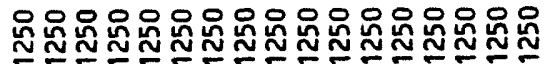

0.0000000000000000000

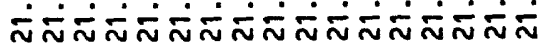




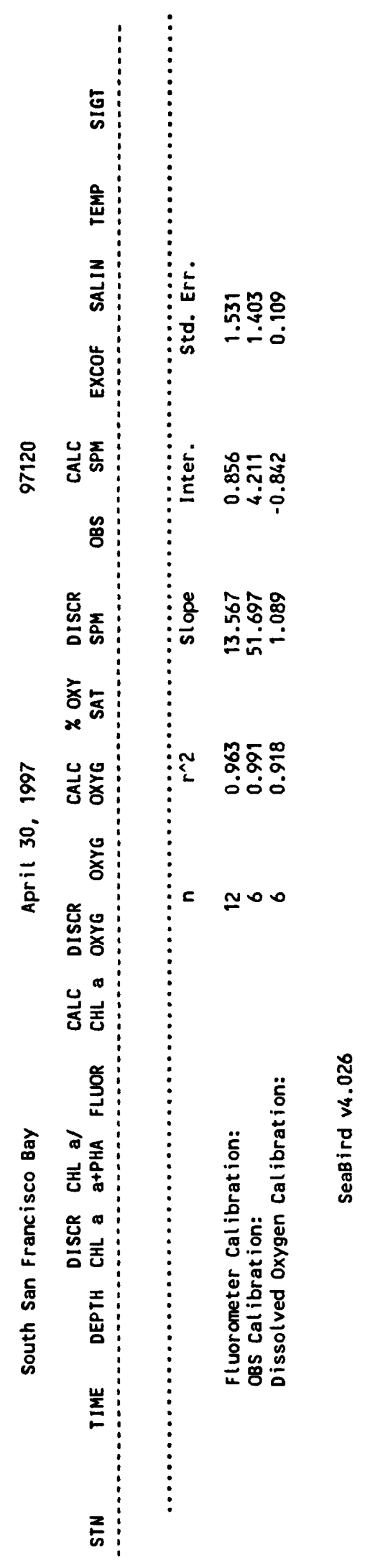




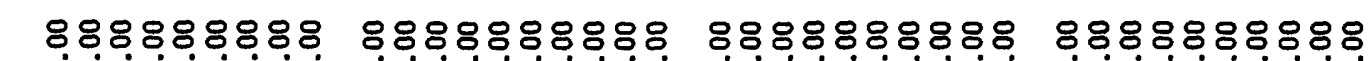

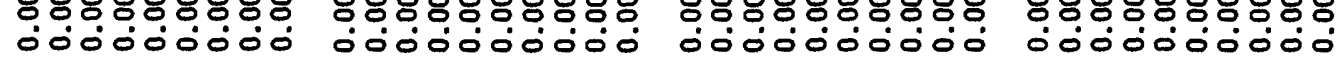

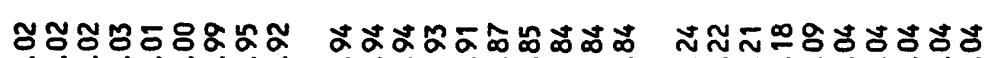

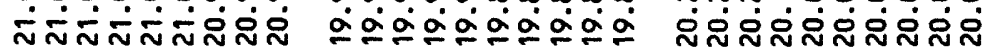

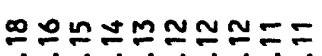
$89890 \%$

으우우우우으으우으으

으으또으으으으 000000000

व்ंல்

000000000

வ்ंீं்

ñ

$\stackrel{\sim}{i}$

$\stackrel{a}{\sim}$

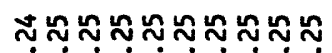

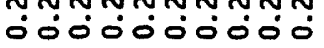

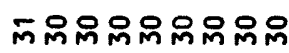

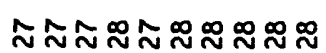

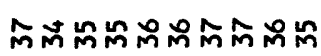

$\stackrel{\circ}{m}$

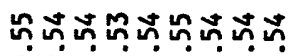

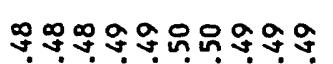

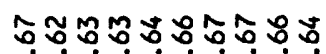
व.0000000

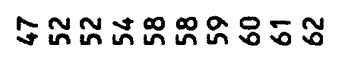

๓ํํㅇํㅇㅇㅇ으느는

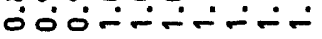

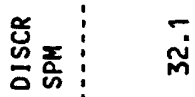
00000000

$$
\text { กั }
$$

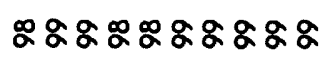

ลำลำลำลำลำลำ

ลำลำลำลำลำลำ

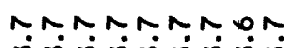
$\infty \infty_{\infty}^{\infty} \infty \dot{\infty} \infty \dot{\infty} \infty \dot{\infty}$

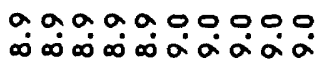

$\sim \sim \infty \sim \sim \infty \infty \infty \infty \infty$

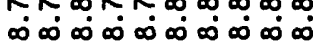

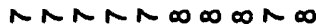
$\infty \infty \infty \infty \infty \infty \infty \infty \infty \infty \infty \infty \infty \infty \infty \infty$

0000000 \% $\infty \infty \infty \infty \infty \infty \infty \infty \infty \infty \infty \infty \infty \infty$

$\infty \infty \infty \infty \infty \infty, \infty \infty \infty$

$00000 \sim \sim \sim N$

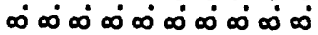
$\infty \infty \infty \infty \infty \infty \infty \infty \infty \infty \infty \infty \infty)$

0000000000 $\infty \infty \infty \infty \infty \infty \infty \dot{\infty} \infty \dot{\infty} \infty \dot{\infty} \infty \dot{\infty}$

$$
\tilde{\infty}
$$

$\stackrel{\infty}{\infty}$

$\stackrel{\infty}{\infty}$

nnmogannand inisinisin

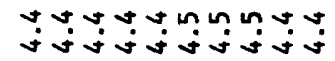

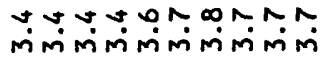

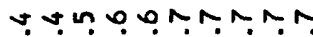

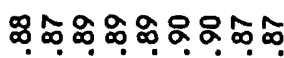

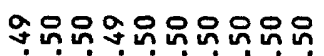

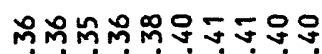
00000000

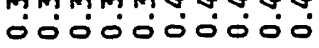

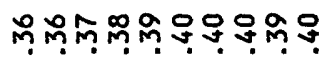
000000000

ஜ̊

$\stackrel{0}{\circ}$

¿

$\stackrel{\infty}{\circ}$

in

i̊

0.0000000

- Nimini ín்

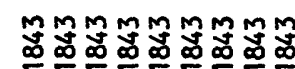

플

웅ㅇㅇㅇㅇㅇㅇ웅

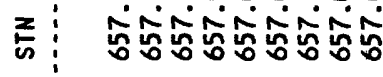

0000000000

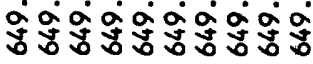

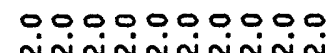

0000000000 


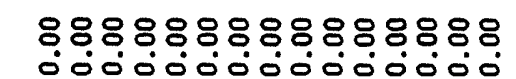

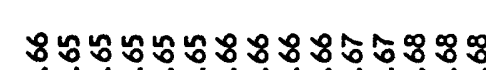

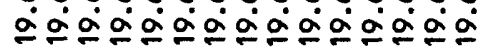

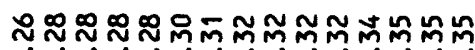

00000000000000

$\stackrel{\Upsilon}{\xi}$

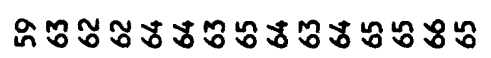

운ำำำกำำำกักำ

呂

芯

ชัญ

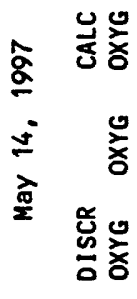

㝴突

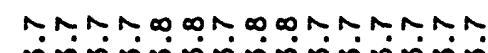

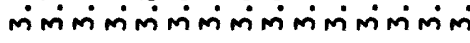

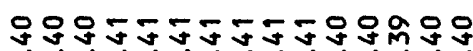
0000000000000

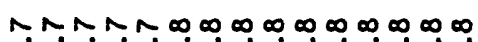
$\infty \infty \infty \dot{\infty} \infty \dot{\infty} \infty \infty^{\dot{0}} \infty \dot{\infty} \infty \dot{\infty} \infty \infty_{\infty}^{\dot{0}}$

๓

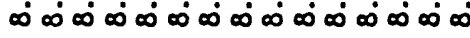

$80.8080 .8080 \div 8$

0000000000

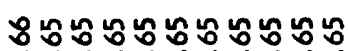

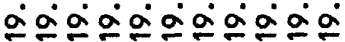

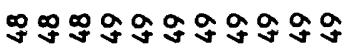

Оं00 000000

in

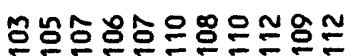

ํำธั์

-ñNiñiñiñ
8888888888

000000000

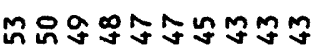

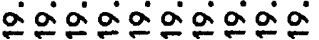

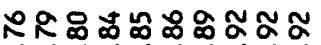

$\because \dot{0}$

$\dddot{a}$

$\stackrel{\sim}{\mathfrak{j}}$

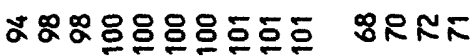

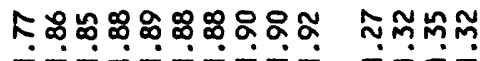

$\stackrel{\infty}{\infty}$ $\dot{\infty} \infty \dot{\infty} \dot{\infty} \dot{\infty} \dot{\infty} \dot{\infty} \dot{\infty} \dot{\infty} \dot{\infty} \infty \dot{\infty} \dot{\infty} \dot{\infty} \dot{\infty} \infty$

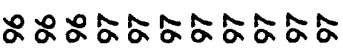

$\sim \infty \infty \infty \infty \infty \infty \infty \infty \infty$ $\infty \infty \infty \infty \infty \infty \infty \infty \infty \infty \infty \infty \infty \infty)$

ONANANNANN

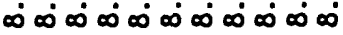

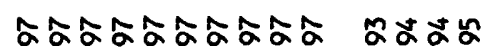

$\infty \infty \infty \infty \infty \infty \infty \infty \infty$ $\dot{\infty} \infty \dot{\infty} \infty \dot{\infty} \infty \dot{\infty} \infty \dot{\infty} \infty$

$m \div$ un $\infty \infty \infty \infty$

QNNANNNAN NmmU $\stackrel{\circ}{\infty}$

?. $\infty \infty \infty \infty \infty \infty \infty \%$

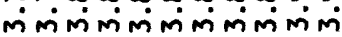

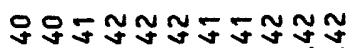

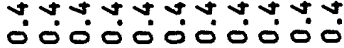

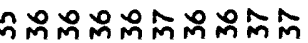
0000000000

m̄nmm ๑๐ं0

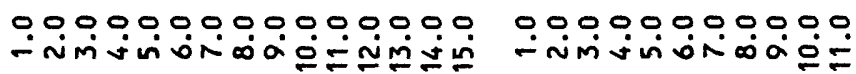

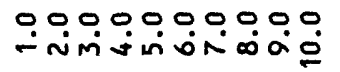

iे

$\dot{m}$

0.00

$\simeq \simeq \simeq \simeq \simeq \simeq \simeq \simeq \simeq \simeq \simeq$

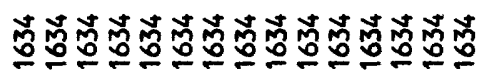

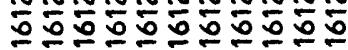

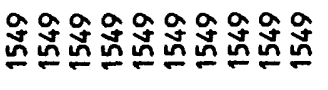

옷유ํำ

\section{)}

끄느는

00000000000 vinisivinisivinising

0000000000

0000

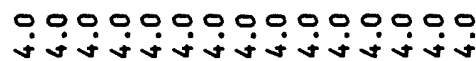




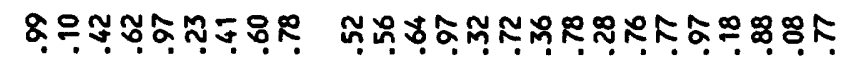

- nininimiming

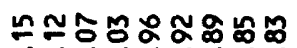

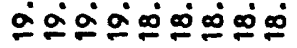

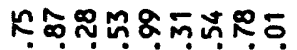

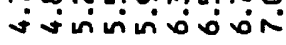

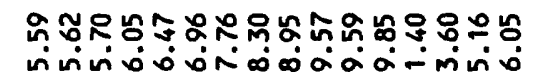

$\stackrel{\infty}{j}$

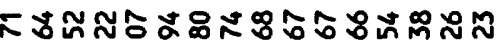

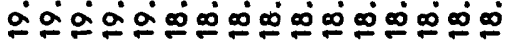

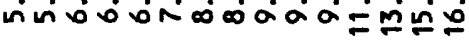

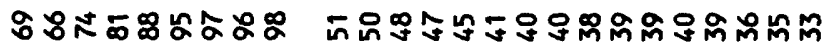

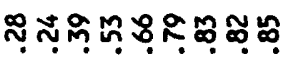

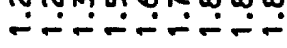

芦

產產

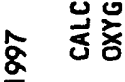

웡

苟

岁론

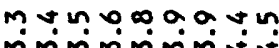
Mñ

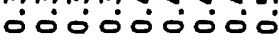

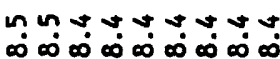

Tmmmmmmmm

$\infty \infty \infty \infty \infty \infty \infty \infty \infty \infty$

옹

호옳

웅 퐁

돔

000000000

n่⿻上丨

0000000000000000

tmm to $000.00 n 0004 n$

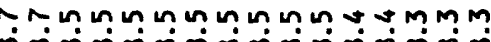

o ondammmmatamnnom

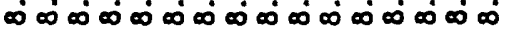

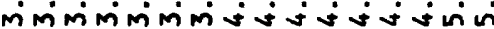

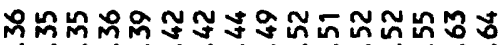
000000000000000

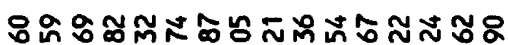
minimiós

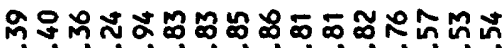

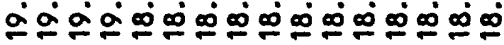

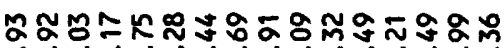

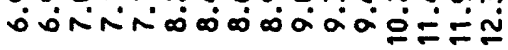

$\tilde{n}$

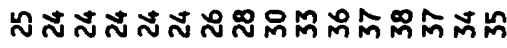

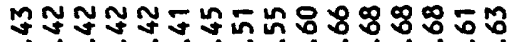
000000000000000

$\tilde{\sim}$

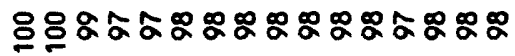

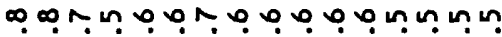
$\infty \infty^{\circ} \infty \infty^{\circ} \infty \infty^{\circ} \infty \infty^{\circ} \infty \infty^{\circ} \infty \infty^{\circ} \infty \infty^{\circ} \infty$

Yัoง

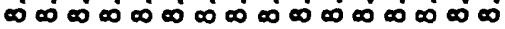

N

mறM

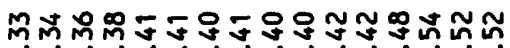
000000000000000

$\stackrel{\circ}{\circ}$

$\stackrel{n}{m}$

0000000000000000

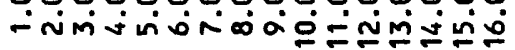

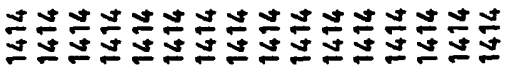

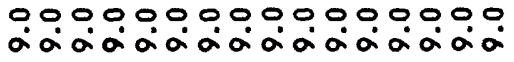

0000000000000000

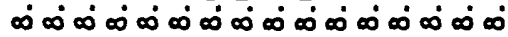




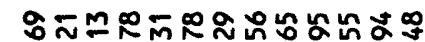

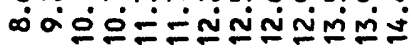

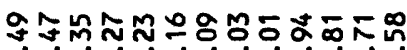

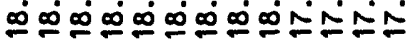

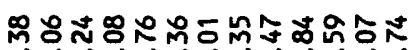

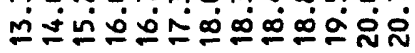

Thina

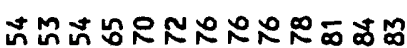
○ं०0ं00000000

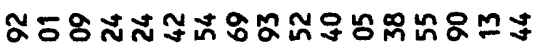

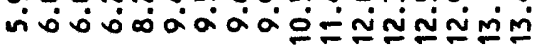

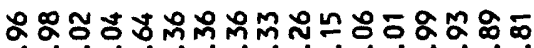

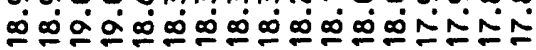

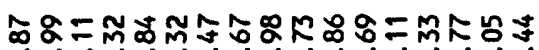

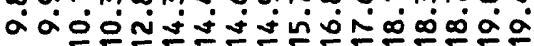

$\bar{n}$

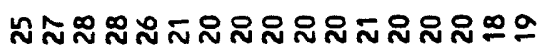

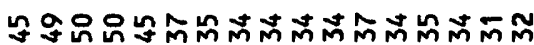
0000000000000000

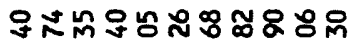

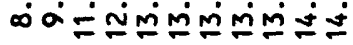

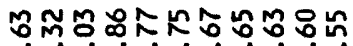

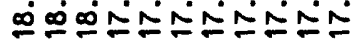
แ่กำสกำㅇำ

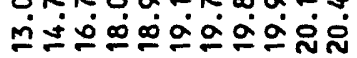
$\dddot{-}$

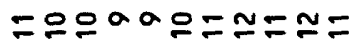

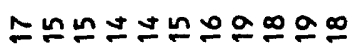
:0ं0ं0ं0்

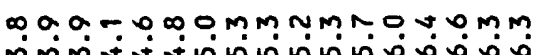

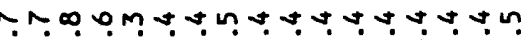
$\infty \infty \infty \infty \infty \infty \infty \infty \infty \infty \infty \infty \infty \infty \infty \infty \infty \infty \infty \infty)$

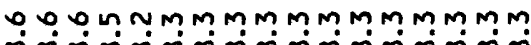

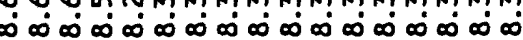

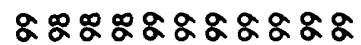

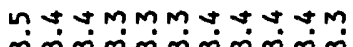
$\checkmark m M \sim N N \sim m M N \sim$ $\infty \infty_{\infty}^{\infty} \infty \infty \infty \infty \infty \infty \infty \infty \infty \infty$

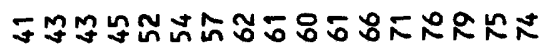

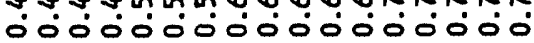

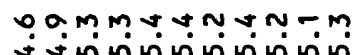

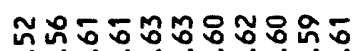

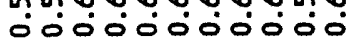

$\stackrel{n}{0}$ $\stackrel{\circ}{\sim}$ 0000000000000

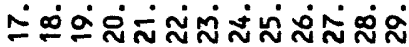

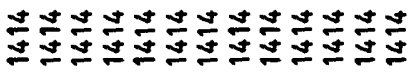

0000000000000 áááåáááá
00000000000000000

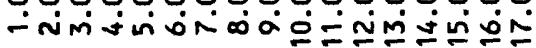

00000000000

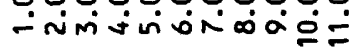

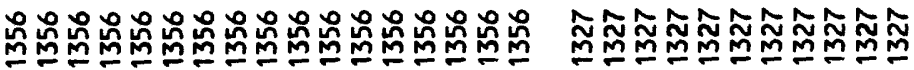

00000000000000000

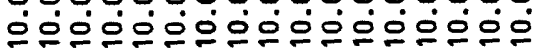

0.000000 .0000

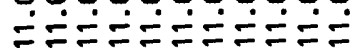




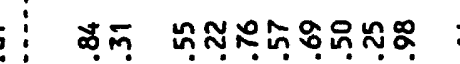

บำ

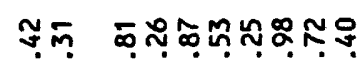

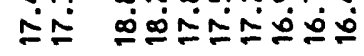

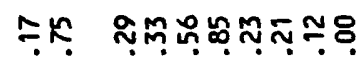

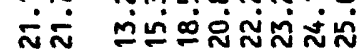

$\stackrel{\dot{0}}{0}$

亲

ฮ̛

$=m$ ONDONOON

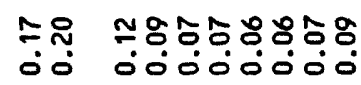

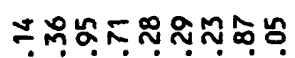

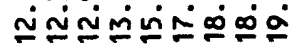

오ำำํำ응

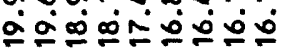

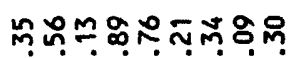

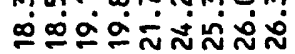

$\stackrel{9}{-}$

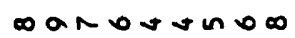

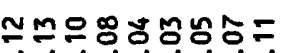
00000000

$\dddot{\sim}$

\section{8}

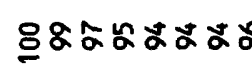

m. on n n.0.0.90

各 考文

$\pm$

高

巡

这䓀

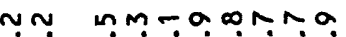

我

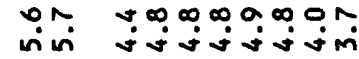

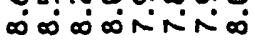

oDonnNan

\section{$\infty$}

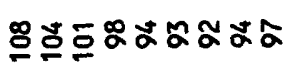

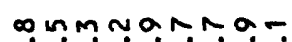

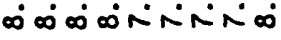

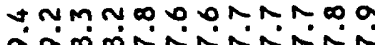

Tan-vonjo

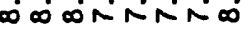

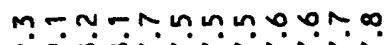

$$
\infty
$$

กำำำำำำำ ó000000000

8ำธธธธัธ 0000000

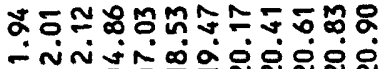

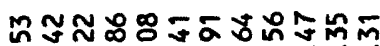

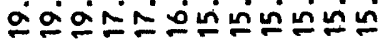

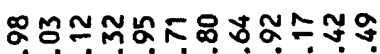

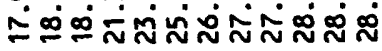

$\stackrel{\infty}{0}$

num nm $r$ ton

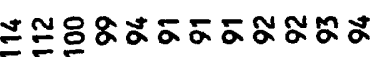

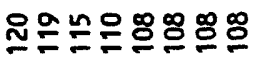

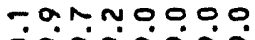

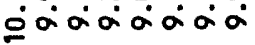

000.090 .90

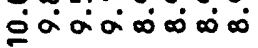

$\check{\circ}$

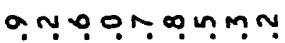

mrरmromogono

univióniso

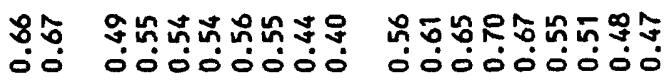

品

i.

$\stackrel{0}{0}$

$\stackrel{\circ}{\circ}$

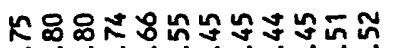
00000000000

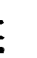

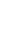

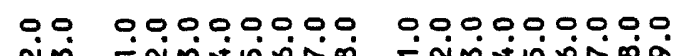

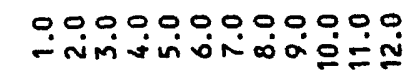

00000000 inivivion

崖

\section{ิㅗำ}

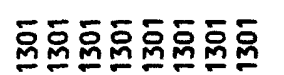

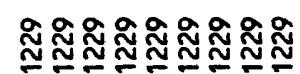

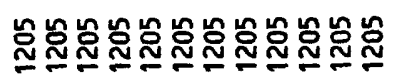

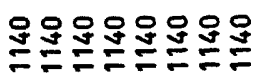

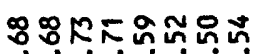
0.000000

$$
\begin{aligned}
& \tilde{\alpha} \\
& 0 \\
& 0 \\
& \infty
\end{aligned}
$$

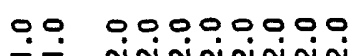

000000000

000000000000

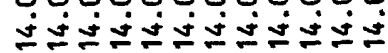

00000000 ํำกำำำำ 


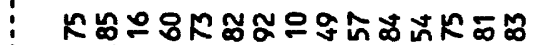

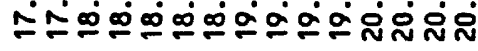

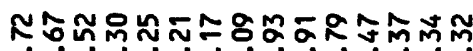

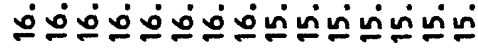

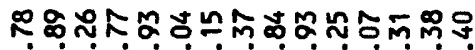

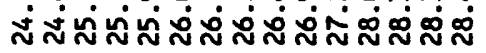

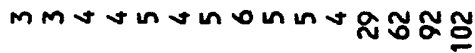

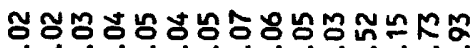

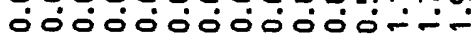

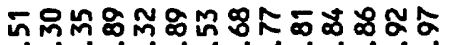

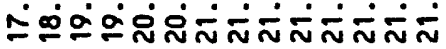

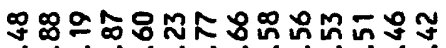

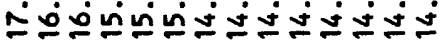

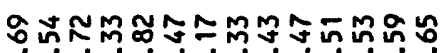

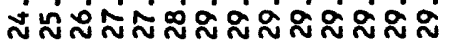
$\stackrel{\circ}{\circ}$

MmmMmMm

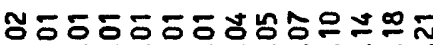

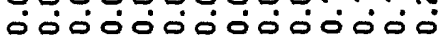

ำำธำำง

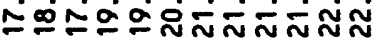

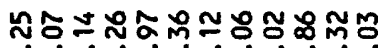

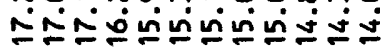

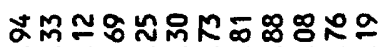

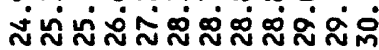

mNNNNmNmNNGo

588885858840 ód0000000

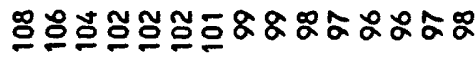

oanonntmmn-..-n

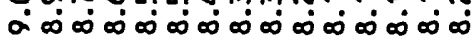

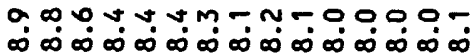

$\infty m 00 m 00-m n-\infty \infty 0 \infty$

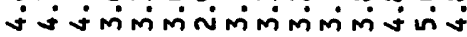

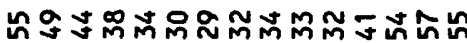
ó000000000000

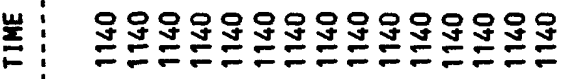

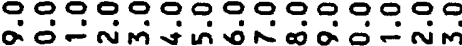

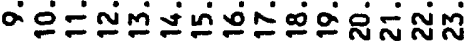

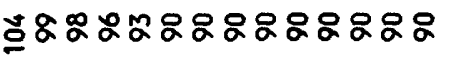

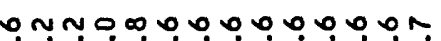


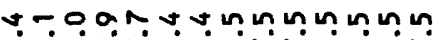

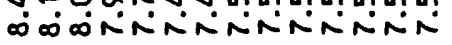

MUNO-NMNO- - N N ن̛ं

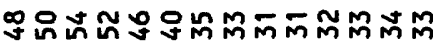

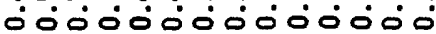

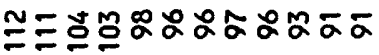

mNoOnم்َ

- - nugagoonon

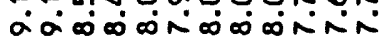

OOUnMn-- nOMO

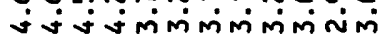

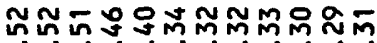
00000000000 $\infty$

$\stackrel{m}{\text { j. }}$

00000000000000 -nims í

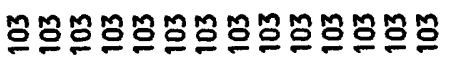

00000000000000

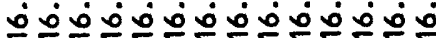

000000000000

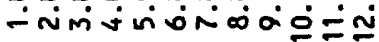

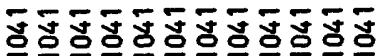

000000000000

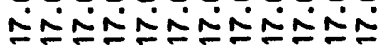




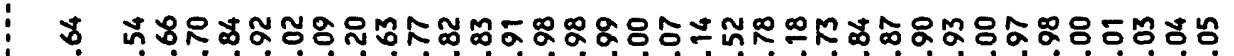

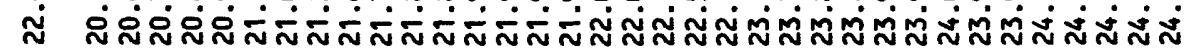

8 ㅇำำำ

ล்亠்̄ं்

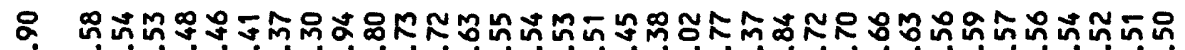

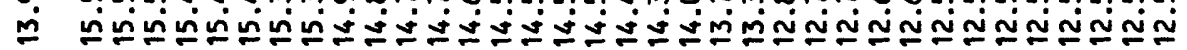

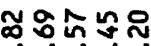

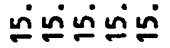

m 을 ํำ

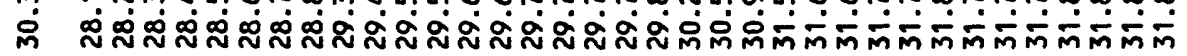

Кฒณ๐ะ

ஸ்

$\dddot{n}$

$\tilde{0}$

a NNNNNNNNNMmUMmmmmunOOM

NonNo

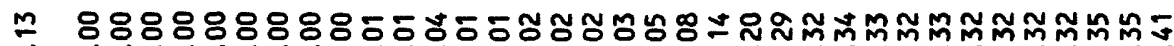

- ó000000000000000000000000000000

808080

0ं0ं0

$\stackrel{9}{2}$

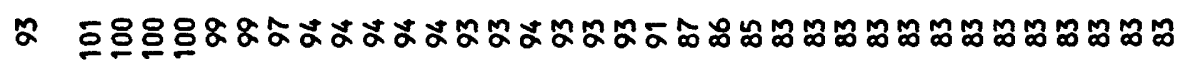

๓ละัณ

- TotmmmnogagagagagandamnNnNmmmmmmmnm চ

$-\infty \infty \infty$

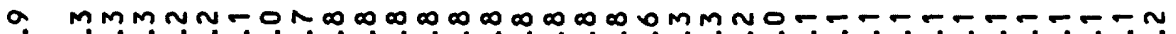

¿

ogan initin

$\dddot{\infty}$

㝴몬

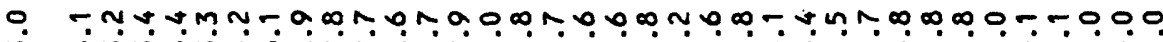

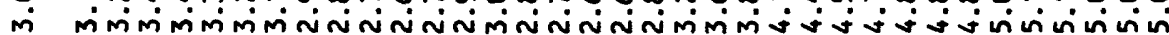

$\infty 0.0+\infty$

miminim

ติ กีmกำ

o.

ษ वं0்

ळ

$\circ$

j

0.00000000000000000000000000000000000

m -

웅ํ요

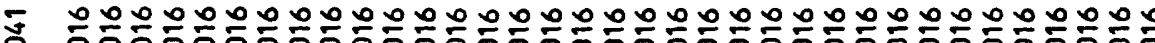

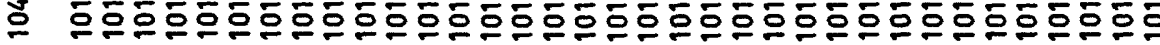

000000000000000000000000000000000000 


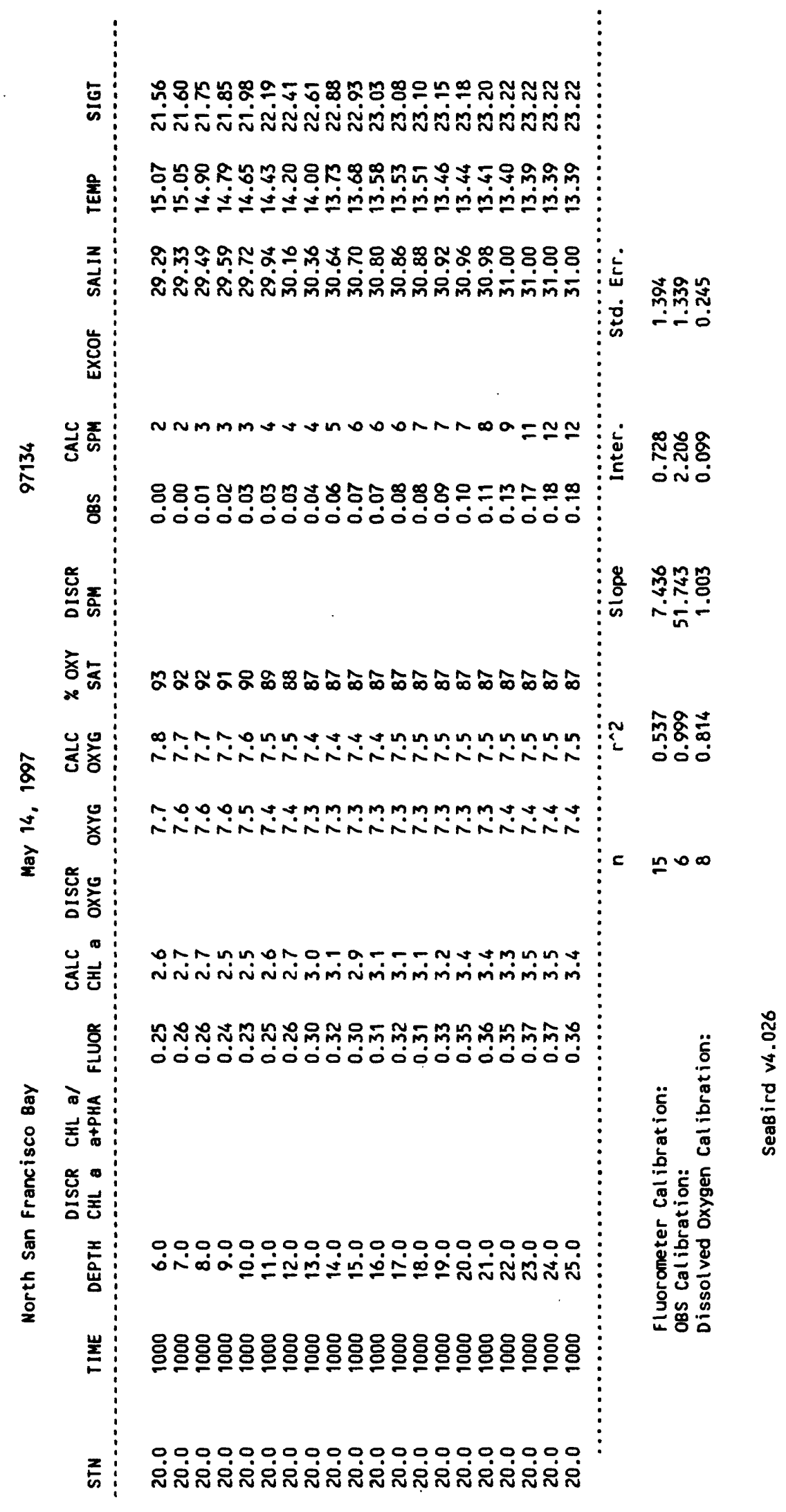




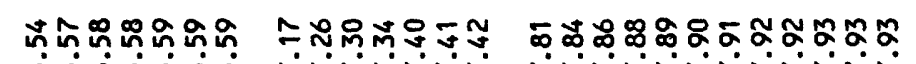

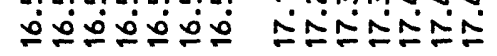

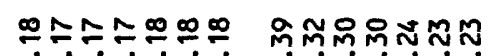

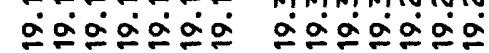

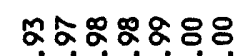

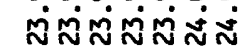
m.

88RกTRD

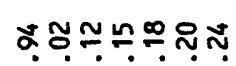

$\dot{8}$

称

i̊

m

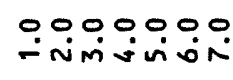

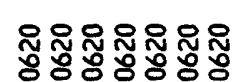

늘

z

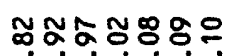
ลัลงฝฝฝ

$\stackrel{\circ}{i}$

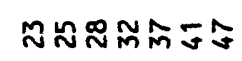

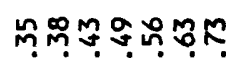
०0000000

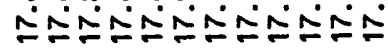

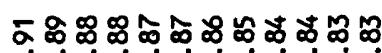

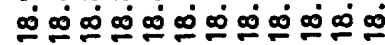

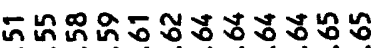

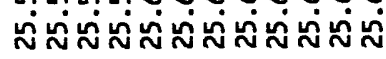

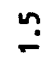

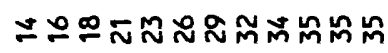

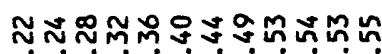

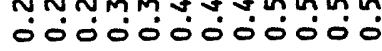<smiles>[CH][CH]</smiles>

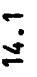

\section{๓亠凶禸} 0000000

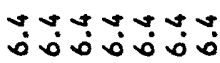

mmmmmmm?

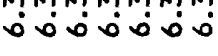

$\frac{0}{0.0000}$

$\tilde{n}$

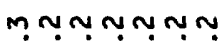

miniminimio

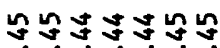

$\bar{\infty} \bar{\infty} \bar{\infty} \bar{\infty} \bar{\infty} \bar{\infty} \bar{\infty} \bar{\infty} \bar{\infty} \bar{\infty}$

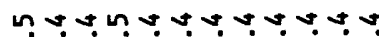
00000000000

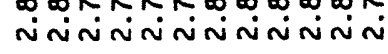

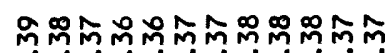
000000000000

:

ì

-

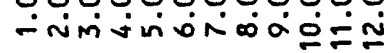

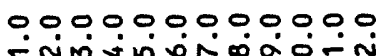

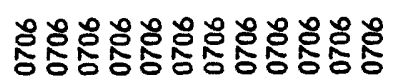

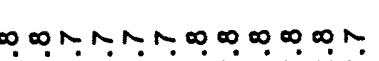

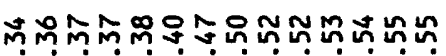

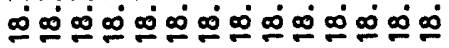

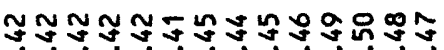

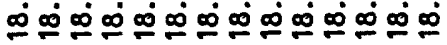

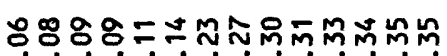

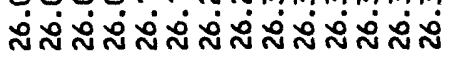

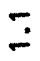

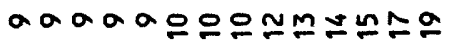

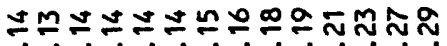

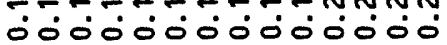

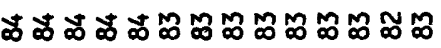

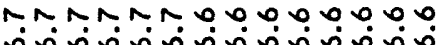

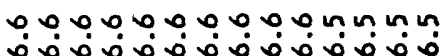
$\hat{0}$

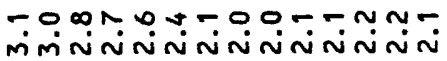

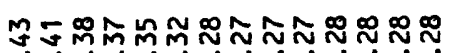
00000000000000

$\begin{array}{ll}\stackrel{0}{0} & \text { ñ. } \\ 0 & \stackrel{0}{n}\end{array}$

00000000000000

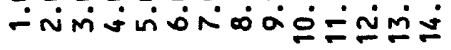

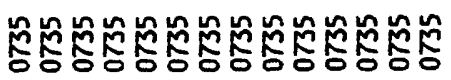

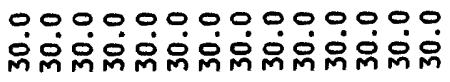
000000000000

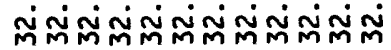




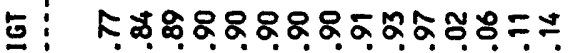

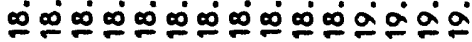

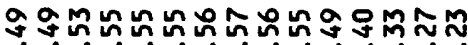

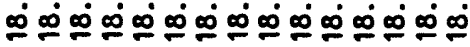

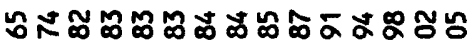

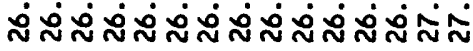

$\stackrel{-}{-}$

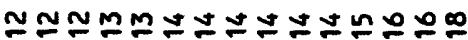

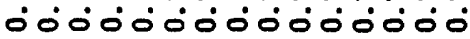

递 爻䒽

mMmmmmMmmMn-- 00

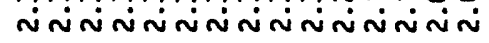

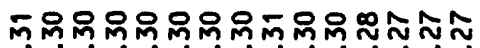
00000000000000

000000000000000

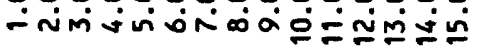
-

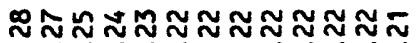

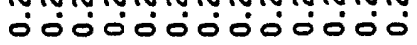

8

$\dot{\sim}$

$\bar{n}$

$\dddot{\Xi}$

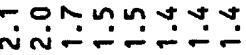

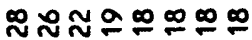
-0000000

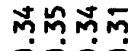
0ं00

กิ

$\dddot{n}$

00000000 - imivioño

0000 - imis

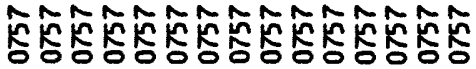

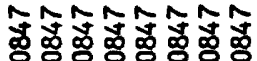

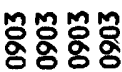

\section{0}

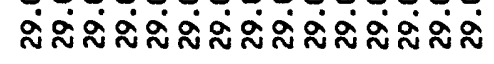

0.000000000000

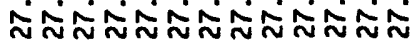

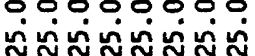

0.00 ป்่ 


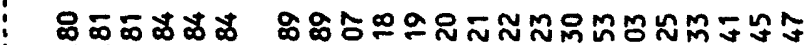

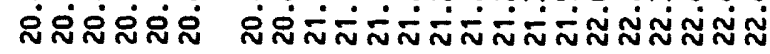

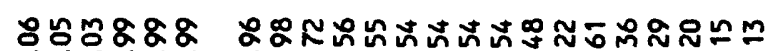

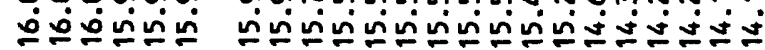

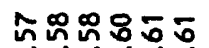

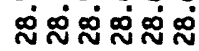

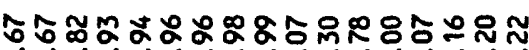

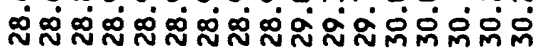

$\stackrel{0}{\circ}$

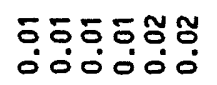

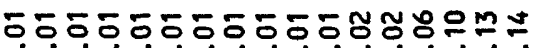

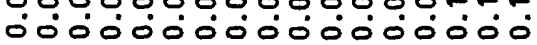

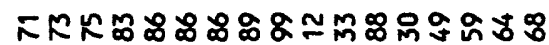

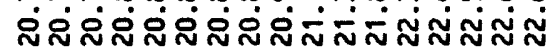

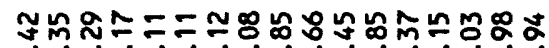

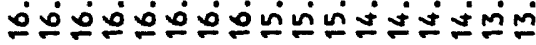

นํํำ

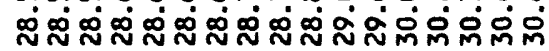

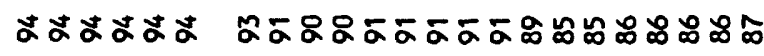

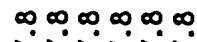
NiNiNin

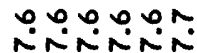
nistañ

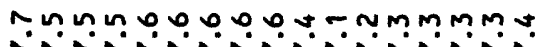

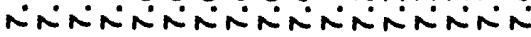

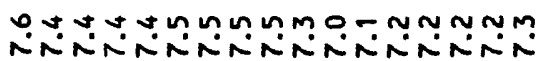
(n)

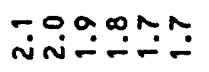

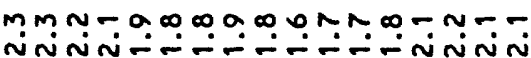

กัก๊ก๊

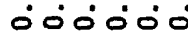

:

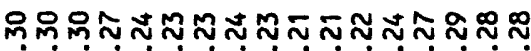
0000000000000000

$\stackrel{\infty}{-}$

눙요영영

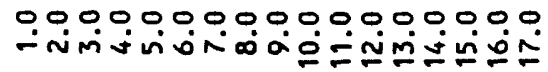

웅영영영영영

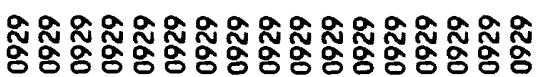

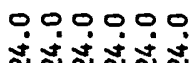

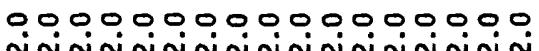

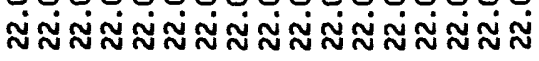

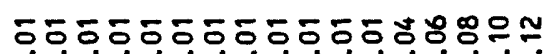

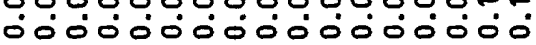

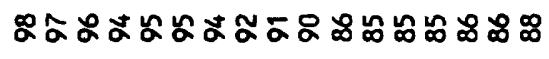

$000 \infty \infty \infty n 00 \backsim n-n m m \div n$

OM

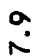

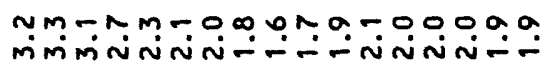

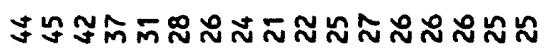
000000000000000

$m$
$\infty$
0

:

$\dot{m}$

$\hat{\sim}$

00000000000000000

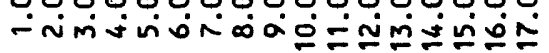

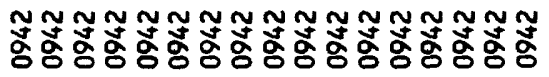

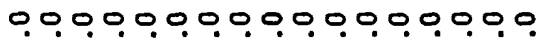

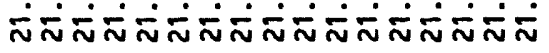




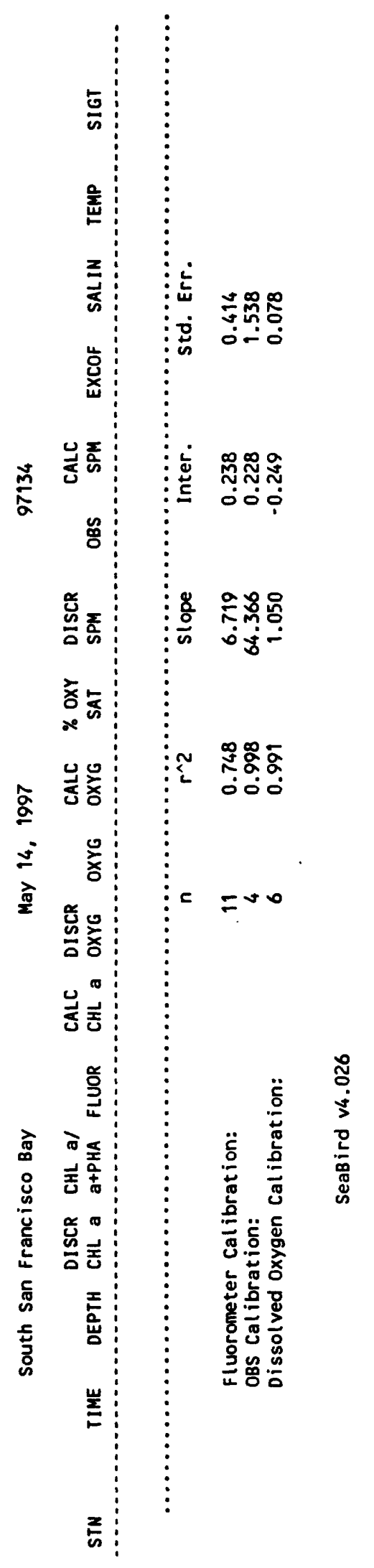


8888888088888888888

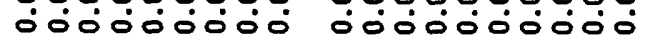

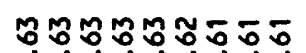

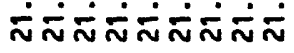

\%

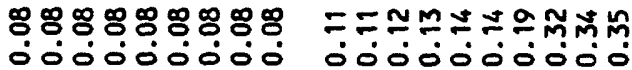

$\stackrel{n}{n}$

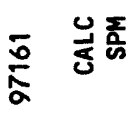

品

总焉

產產

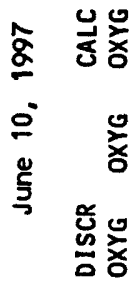

옹론

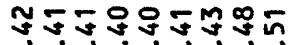
000000000

$\dot{m}$

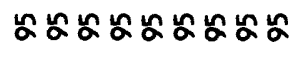

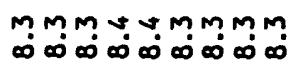

mmmmmmmmm

$\infty \infty_{\infty}^{\infty} \infty \dot{\infty} \boldsymbol{\infty} \infty \dot{\infty} \infty \dot{\infty} \boldsymbol{\infty}$

$\stackrel{\infty}{\infty}$

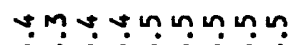
minimimimimimim

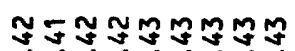

00000000

సั.

mó

$\stackrel{\infty}{i}$

웅 몽

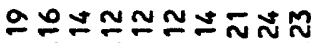

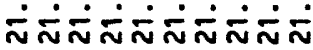

$\stackrel{\circ}{m}$

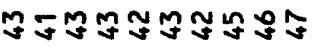

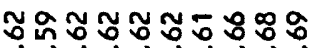
000000000

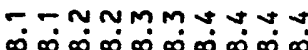
$\stackrel{n}{\infty}$

$\because-\neg-0, \infty \infty$

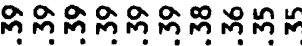
0000000000

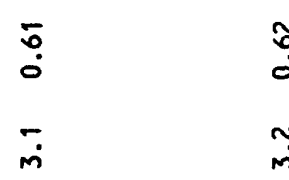

웅ㅇㅇㅇㅇㅇㅇㅇㅇㅜ

웅ㅇㅇㅇㅇㅇㅇㅇ웅

-nimisiñó
88888888888

0000000000

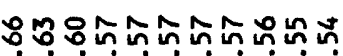

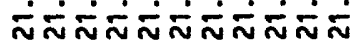

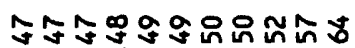

00000000000

$\stackrel{a}{i}$

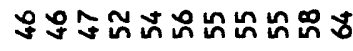

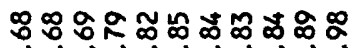
0000000000
888888888

í000000

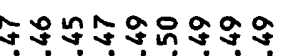

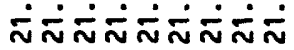

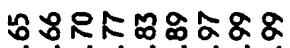
000000000

$\stackrel{+}{m}$

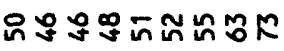

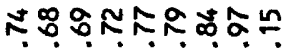
00000000-

$\stackrel{\leftrightarrow}{m}$

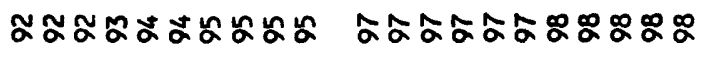

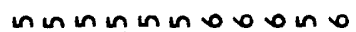
$\infty \infty \infty \infty \infty \infty \infty \infty \infty \dot{\infty} \infty \dot{\infty} \infty \dot{\infty}$

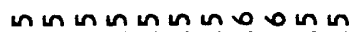
$\infty \infty_{\infty}^{\infty} \infty \infty_{\infty}^{\infty} \infty \infty_{\infty}^{\infty} \infty \infty_{\infty}^{\infty}$

T. . iniñninininin

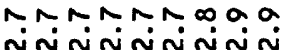

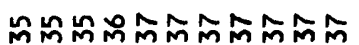

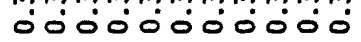

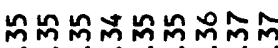
ó000்00்

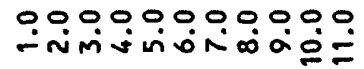

:ัّ

$\underset{0}{\infty}$

$\tilde{n}$

$\bar{m}$

운웅요웅영

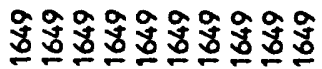

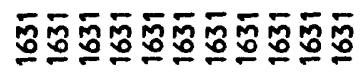

ํํำำำำำ 드은두은

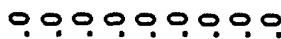

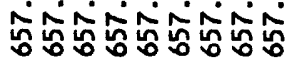

0000000000

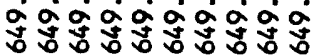

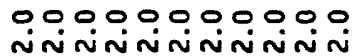

000000000 mंmimmimimi 


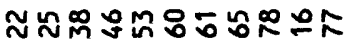

inำ

¿000000000000

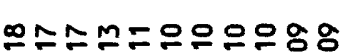

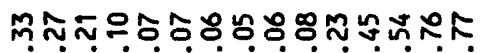

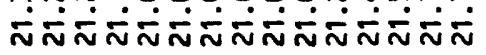

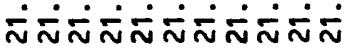

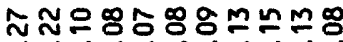
กิก

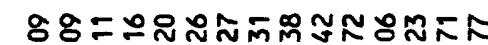

NลNהNKR№k

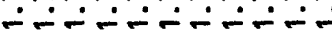

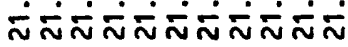

ฉะิ

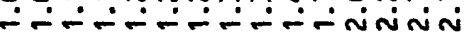

$\stackrel{\leftrightarrow}{m}$

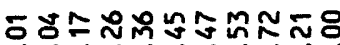

miniminiminimion

ธูกร

$\dot{m}$

$\stackrel{\circ}{n}$

$\dddot{v}$

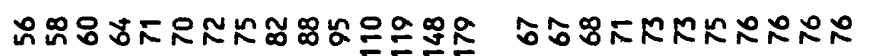

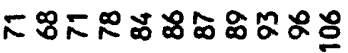

ก゚๊

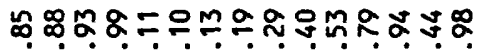

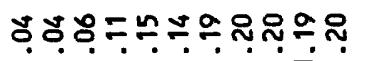

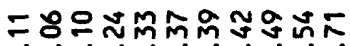

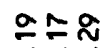

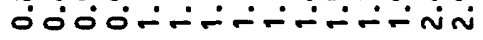

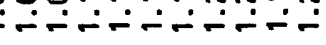

งุ่

爻產

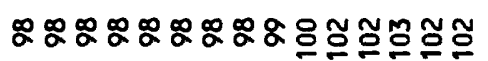

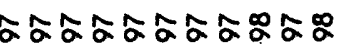

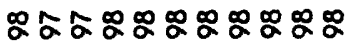

ะะ

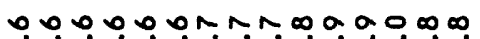

แn un un un in in 00 in

ก. แn แn แn แn แn 0 แn un $\infty \infty_{\infty}^{\infty} \infty \dot{\infty} \infty \dot{\infty} \infty \dot{\infty} \infty \dot{\infty} \infty \dot{\infty} \dot{\infty} \infty \dot{\infty}$ $\infty \dot{\infty} \infty \dot{\infty} \infty \dot{\infty} \infty \dot{\infty} \infty \dot{\infty}$

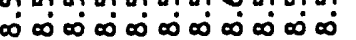

บ $N$ $\infty \infty \infty$

$0000,0, N \infty 000 \infty N$

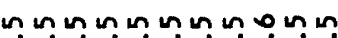

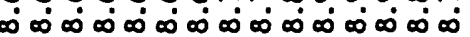
$\dot{\infty} \dot{\infty} \dot{\infty} \dot{\infty} \infty \dot{\infty} \dot{\infty} \dot{\infty}$

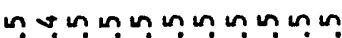
$\infty \dot{\infty} \infty \dot{\infty} \infty \dot{\infty} \infty \dot{\infty} \infty \dot{\infty} \infty \dot{\infty}$

บำ $\infty \infty \infty$

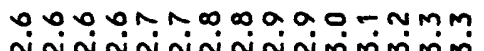

mommotannumun

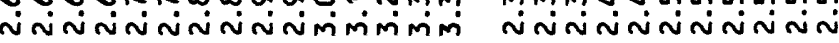

- 0 iñininininin

$\infty \infty \infty$

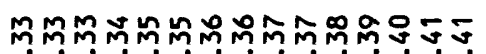

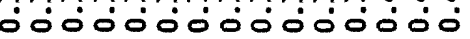

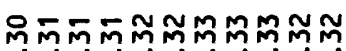

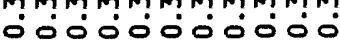

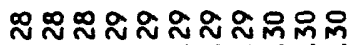
ல0ं0்0ं0்00

$\aleph ๗$

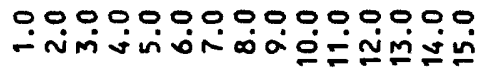

00000000000

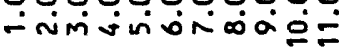

กี่

$\bar{\sigma}$

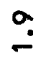

$\dddot{n}$

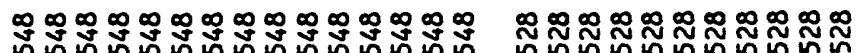

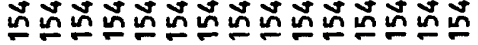

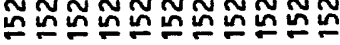

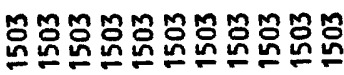

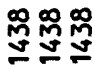

00000000000000000000000000

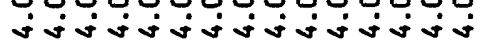

niviniviniminivinim

00000000000

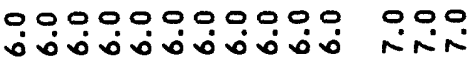




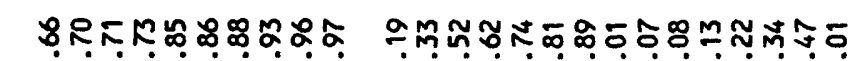

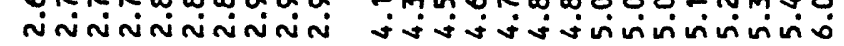

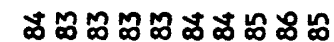

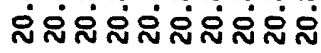

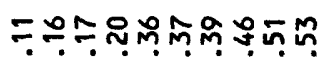

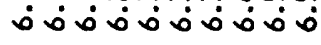

:

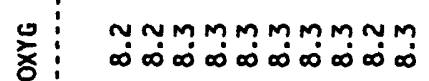

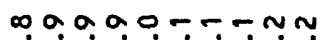

-i-ninininin

ะกกำณำำำ ஸ்0ல000ல0ல

กิ

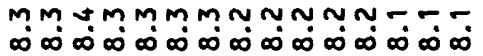

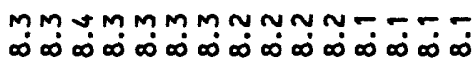
$\infty$

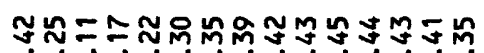

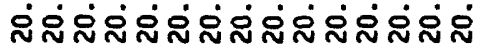

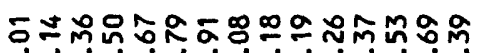

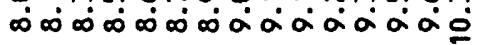
in

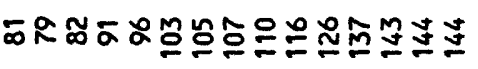
ลิฝ昂

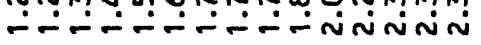

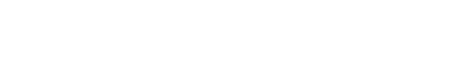

n- $00000000-n$ nnNinininininininiñiñ

ని జ 000000000000000

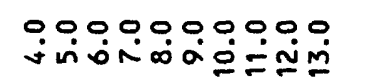

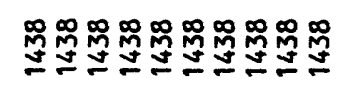

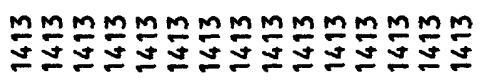

000000000000000

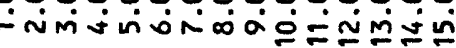

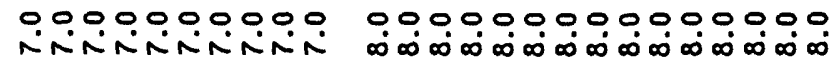

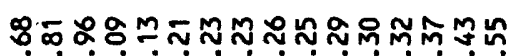

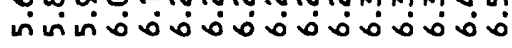

ใ은

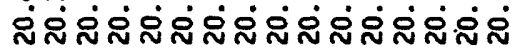

象 స -

$\stackrel{\sim}{\mathfrak{j}}$

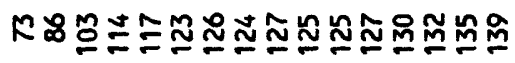

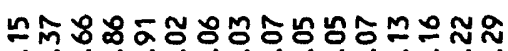

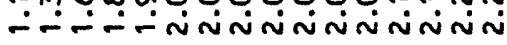

$\dot{1}$

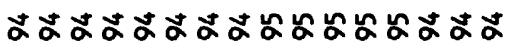

0000000000000000

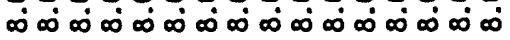

0000000000000000

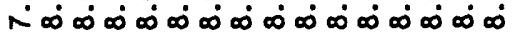

$$
\infty
$$

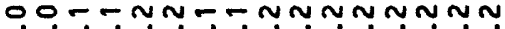

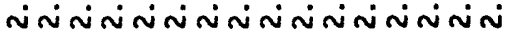

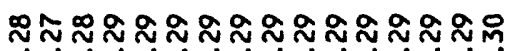
00000000000000

$$
\begin{aligned}
& \stackrel{0}{0} \\
& 0 \\
& \stackrel{\infty}{0}
\end{aligned}
$$

0000000000000000

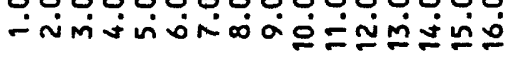

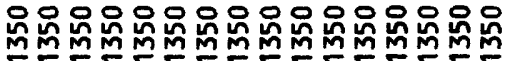

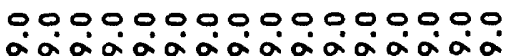




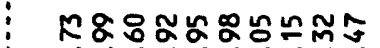

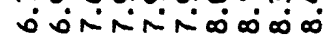

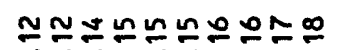

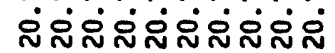

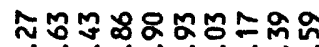

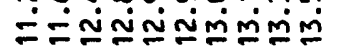

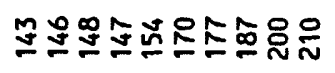

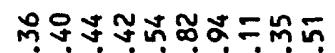
iñNiñnimimio

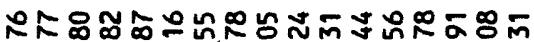

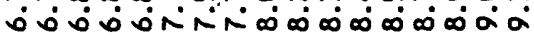

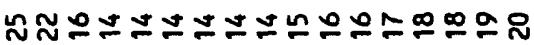

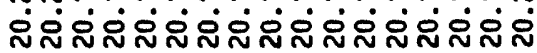

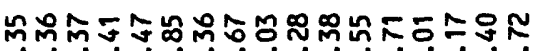

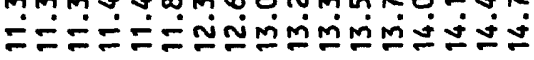
in

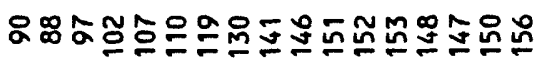

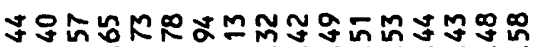
-

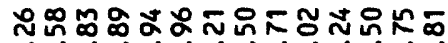

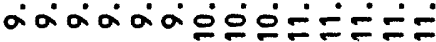

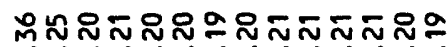

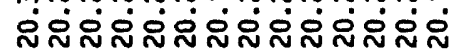

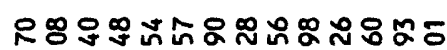

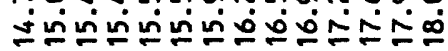
$\stackrel{0}{j}$

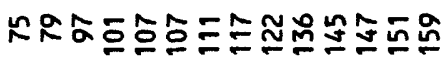

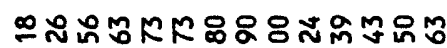
-

$0000009 \infty \infty \infty \infty \infty \infty+\infty)$ $0, \infty \infty \infty \infty \infty \infty \pi n$

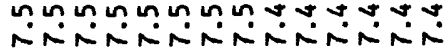

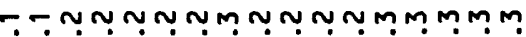
inininininino

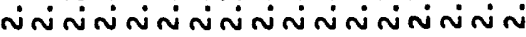
웄ํํํํำตำกำ

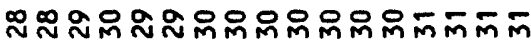
0000000000 00000000000000000

00000000000000 -

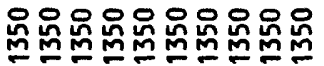

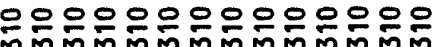

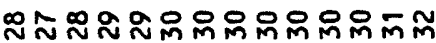
0000000000000 


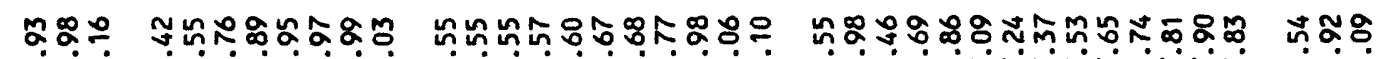

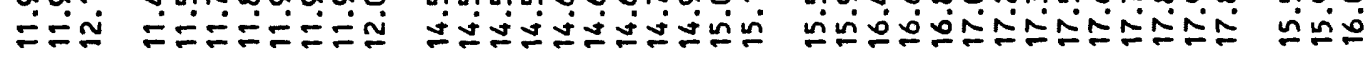

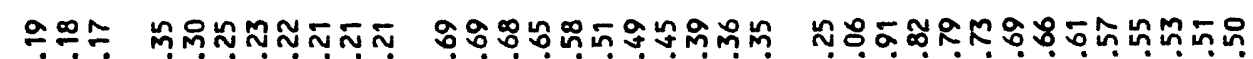

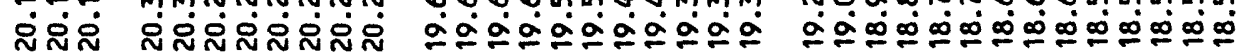

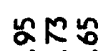 $\stackrel{\infty}{\infty} \dot{\infty}$

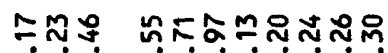

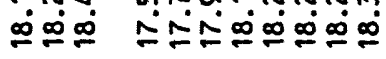

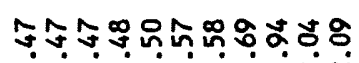

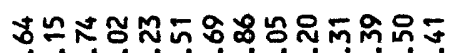

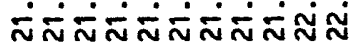 \\ กับ \\ in

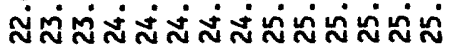 \\ ำ \\ กิก่}

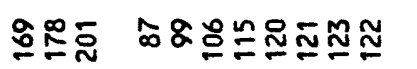

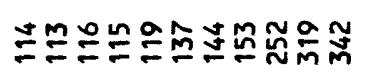

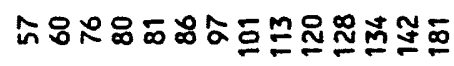

กำำ

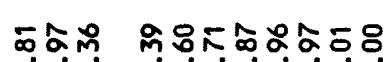

نñm

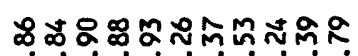

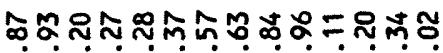

อง

$\stackrel{a}{\simeq}$

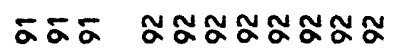

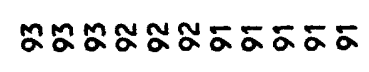

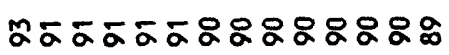

ะสะ

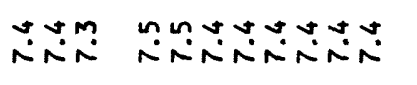

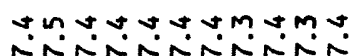

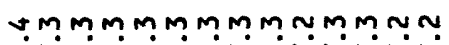

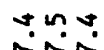

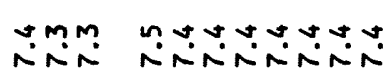

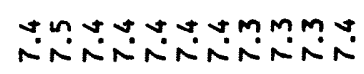

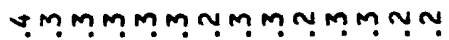

iñ

$\stackrel{n}{\Delta}$

ก

กำ mmymutunn

opopoponompon

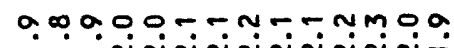

iุīi

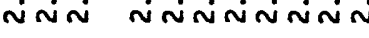

Niñininimimm

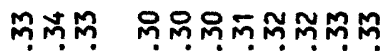

O०0 00000000

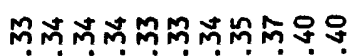

0000000000

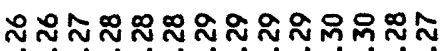

000000000000

กิกฺฺุ

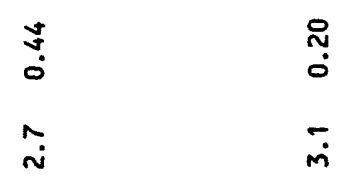

m.

$\stackrel{0}{\circ}$

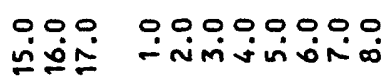

웅우웅우웅웅

00000000000000

000

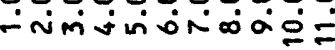

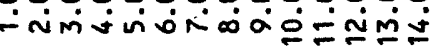

$\therefore$ in

崖

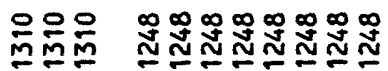

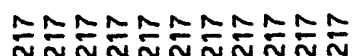

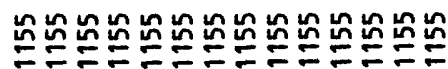




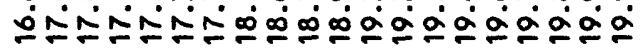

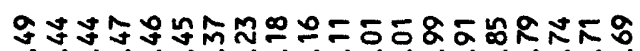
ஸ⿻

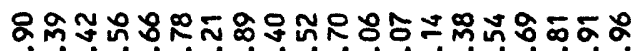

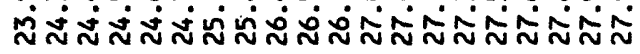

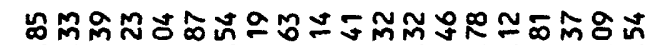

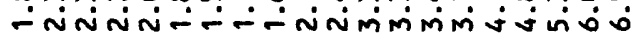

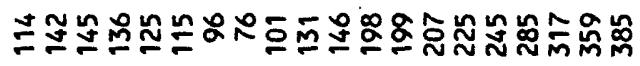

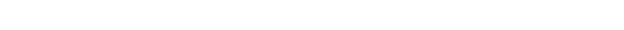

递密

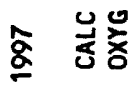

¿ รั)

雚

爮宝

Tr- -00000,-manonomonat

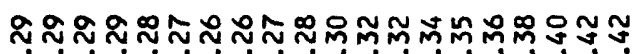
-00000000000000000

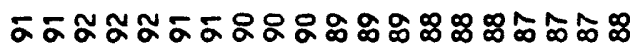

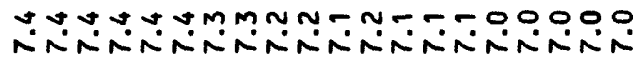

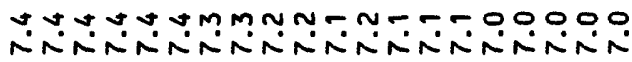

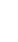

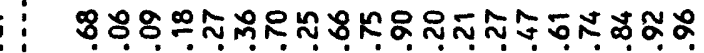

岁

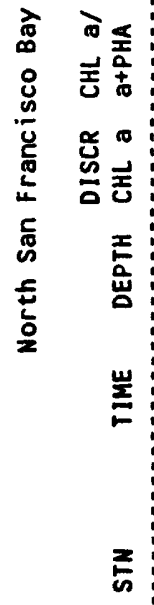

00000000000000000000

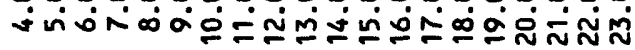

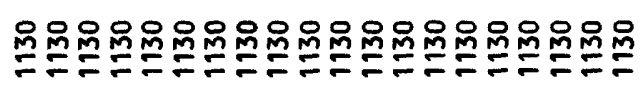

00000000000000000000

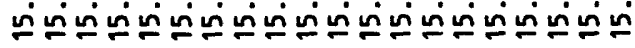

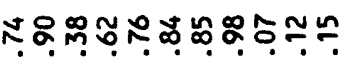

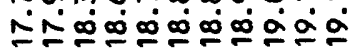

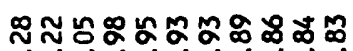

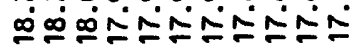

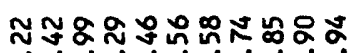

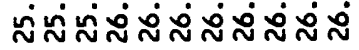

$\dot{m}$

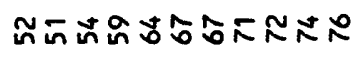

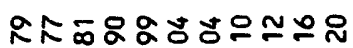

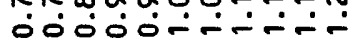

$$
\stackrel{q}{g}
$$

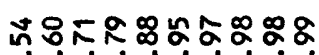

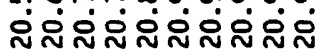

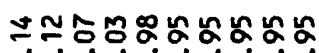

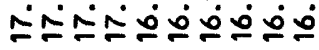

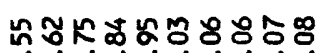

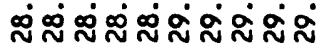

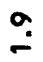

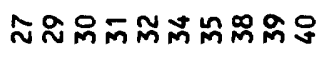

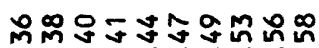
0000000000

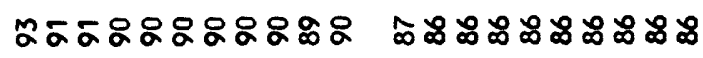
mommmnNMNn $000 a a a 0 a a a$

m

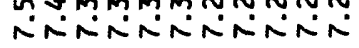

0000000000

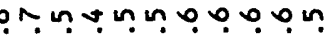

○

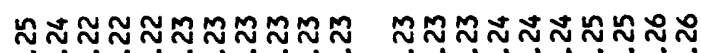

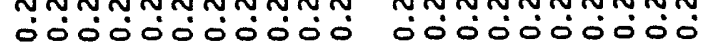

00000000000

0000000000 - Nimivioños=

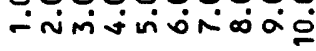

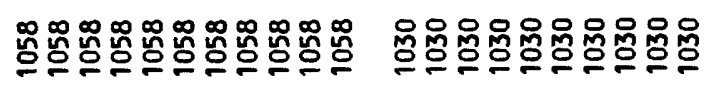

00000000000

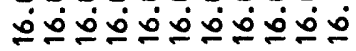

0000000000

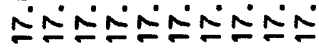




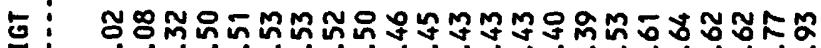

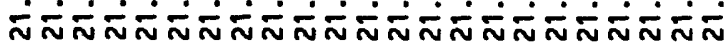

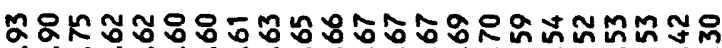

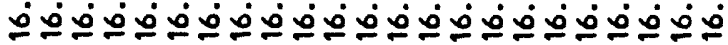

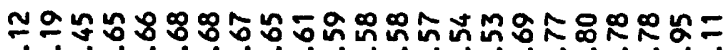

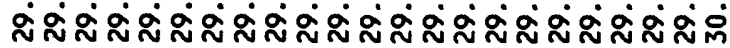

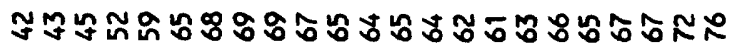

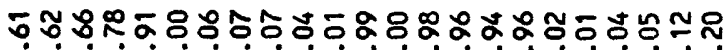

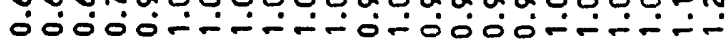

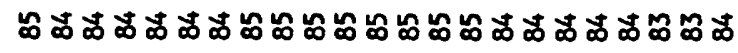
$0, \infty \infty \infty \infty, 0,0,0,0,0,0, \infty \infty, \infty \infty \infty \infty$ $\dot{0} \dot{0} \dot{0} \dot{0} \dot{0} \dot{0} \dot{0} \dot{0} \dot{0} \dot{0} \dot{0} \dot{0} \dot{0} \dot{0} \dot{0} \dot{0} \dot{0} \dot{0} \dot{0} \dot{0} \dot{0} \dot{0} \dot{0}$ $0, \infty \infty \infty \infty \infty, 0,0,0,0,0, \infty \infty, \infty \infty \infty$

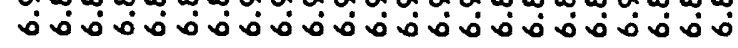
ชิ :

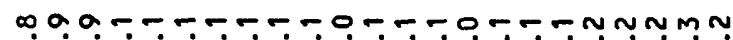

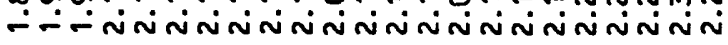

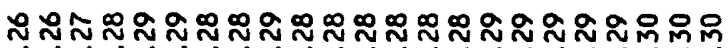

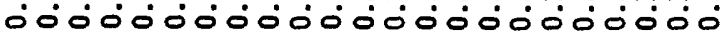

\section{0

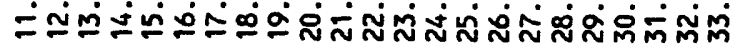

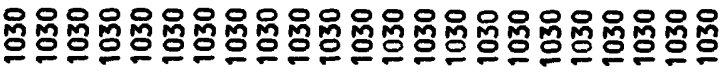

000000000000000000000000

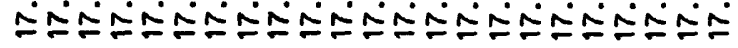

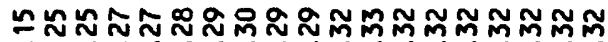

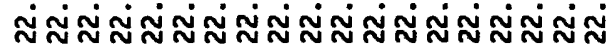

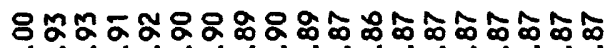

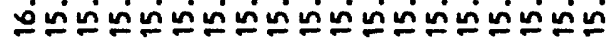

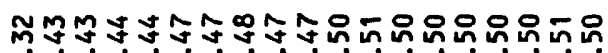

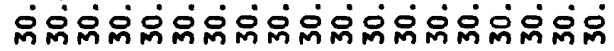
$\ddot{m}$

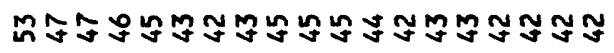

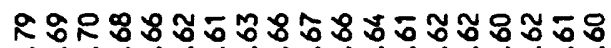
வ்

$\stackrel{\infty}{m}$

mo

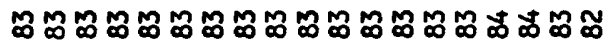

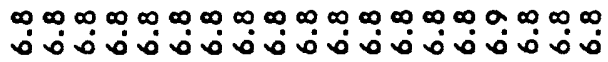

$\infty \infty \infty \infty \infty \infty \infty \infty \infty \infty \infty \infty \infty \infty \infty \alpha \infty \infty \infty$ 遂

$\stackrel{\infty}{\dot{0}}$

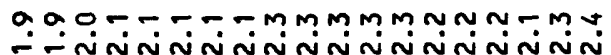

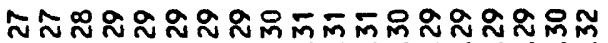
0ं0ं00000000000000<smiles>[CH]=[SiH2]</smiles>

$\stackrel{\circ}{\text { i }}$

0000000000000000000

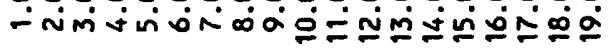

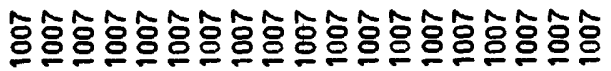

0000000000000000000

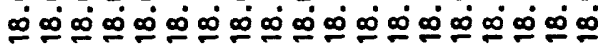




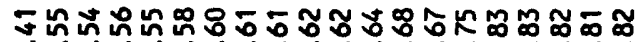

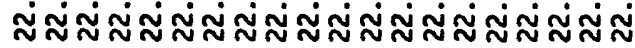

届

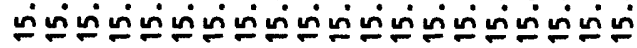

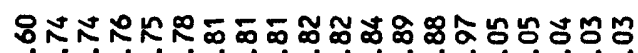

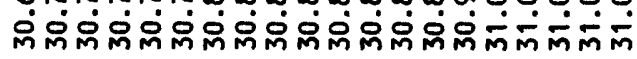

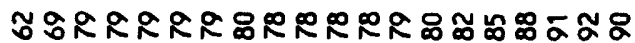
ó0000000000000000

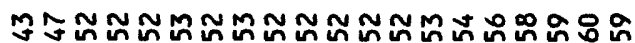

总

爻衰

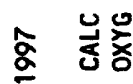

웛론

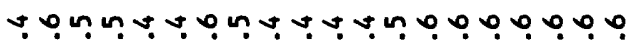

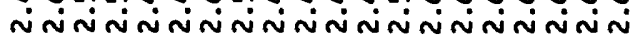

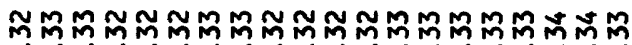
0000000000000000000

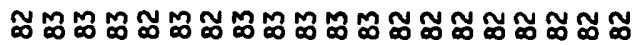

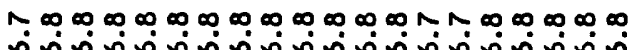

$\infty \infty \infty \infty \infty \infty \infty \infty \infty \infty \infty \infty \uparrow, \infty \infty \infty \infty \infty$

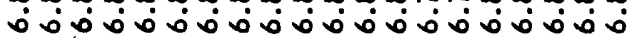

00000000000000000000

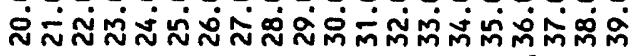

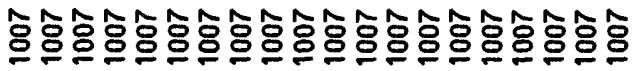

00000000000000000000

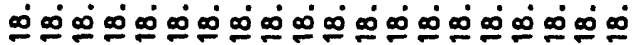

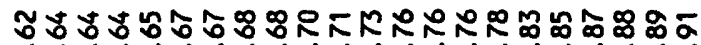

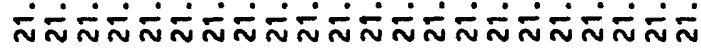

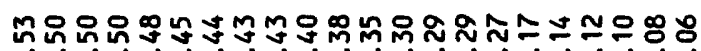

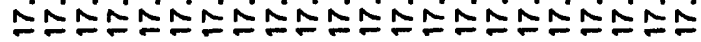

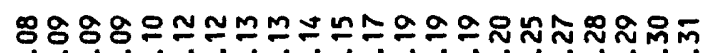

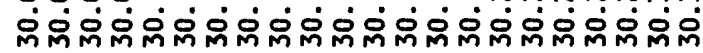

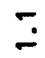

ㅇํํํㅇำก

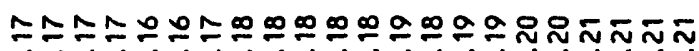

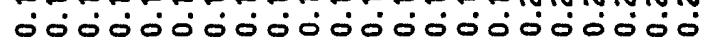

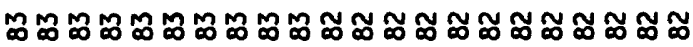

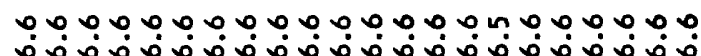
0000000000000004000000

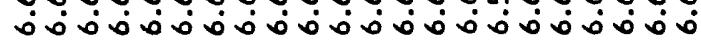

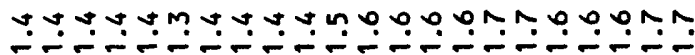

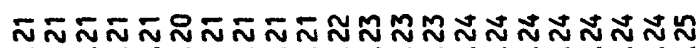
0000000000000000000

0000000000000000000000

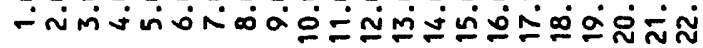

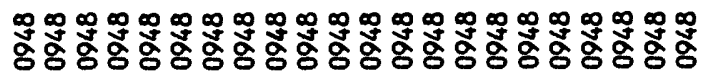

0000000000000000000000

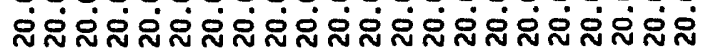




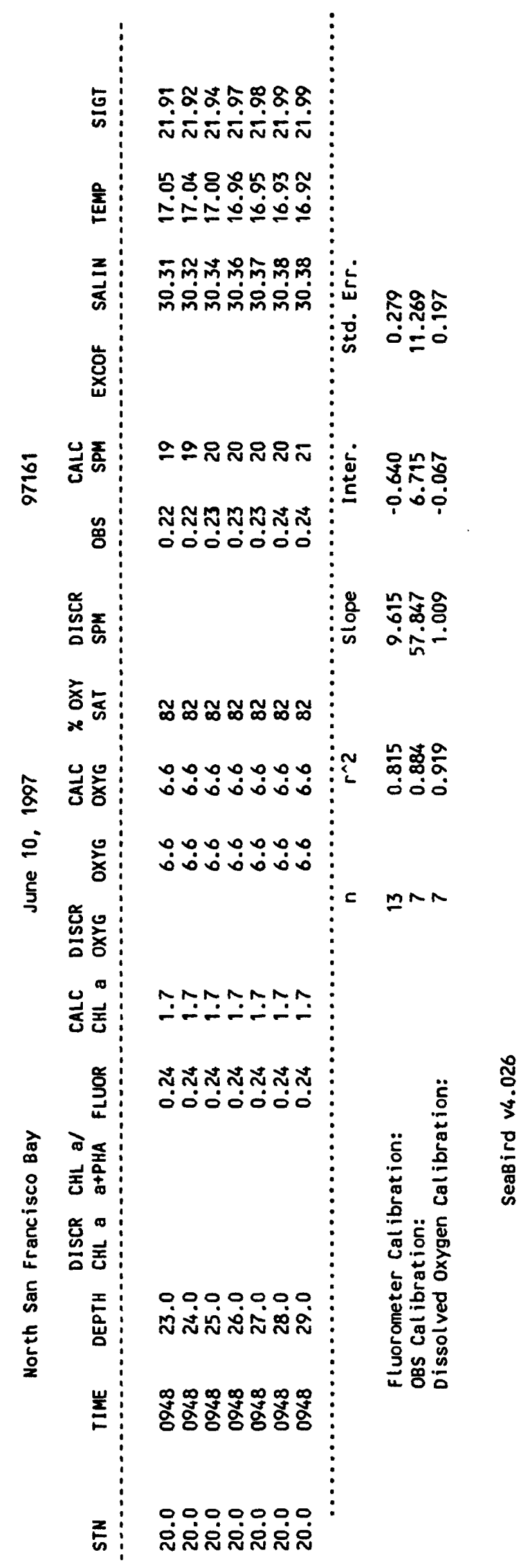




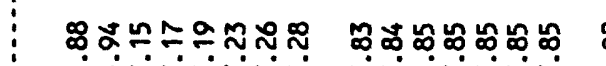

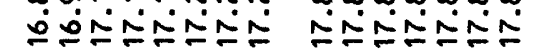

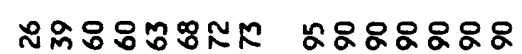

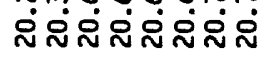

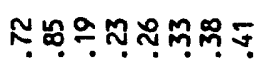

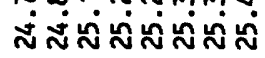

$$
\text { m }
$$

ละกลกกลก

국워원워

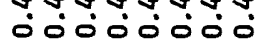

$\stackrel{\infty}{\infty}$

๕ธะ๕8ะ๕ณ

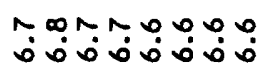

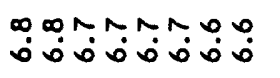

$$
\stackrel{\circ}{0}
$$

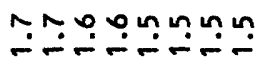

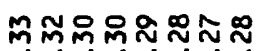

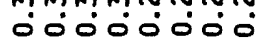

ก

ז.

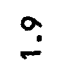

윰퐁

क

ڤ̊

\section{ฉฉฉะ8ะล}

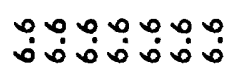

OMNNNNN 0000000

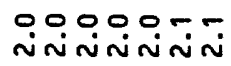

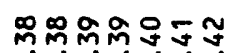

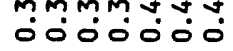

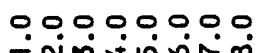

웅우웅웅

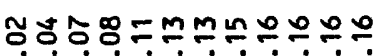

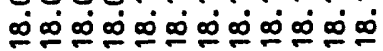
กิกิกักิธ่

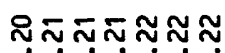

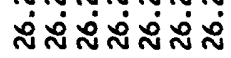

$\bar{i}$

กำลำกับ

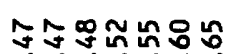

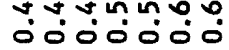

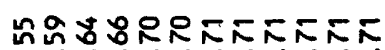

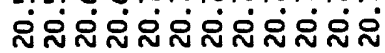

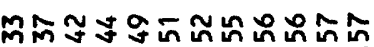

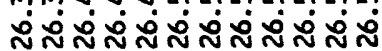

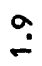

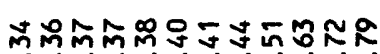

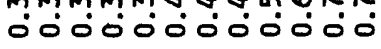

タ

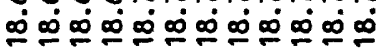

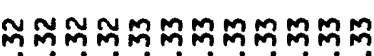

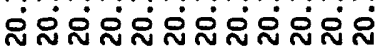

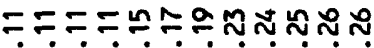

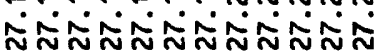
$\cong$

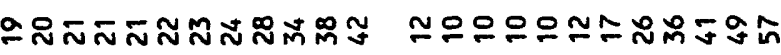

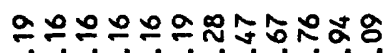

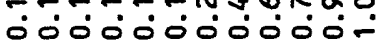

$$
\ddot{\circ}
$$

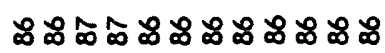

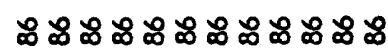

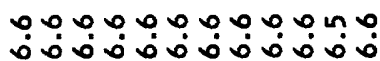

000000000000

NMNMNMNOOOOO

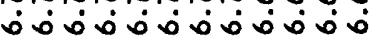

Trannarano

$$
\stackrel{\circ}{\circ}
$$

$$
\stackrel{\circ}{0}
$$

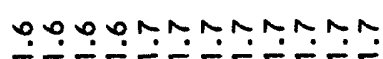

บฺ̣กฺฺฺฺmmmmm

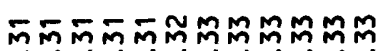

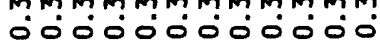

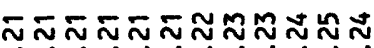

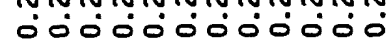

$:$
$:$

$\begin{array}{lll}m & \stackrel{0}{0} & \stackrel{0}{0} \\ 0 & 0 & \stackrel{0}{0}\end{array}$

000000000000

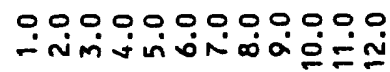

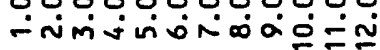




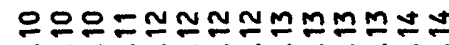

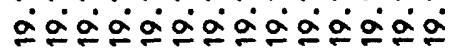

ニニ

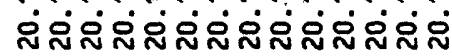

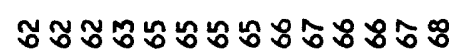

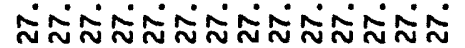
?

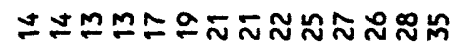

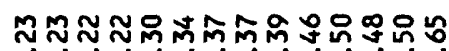
000000000000

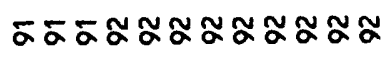

$\operatorname{lin} 0$

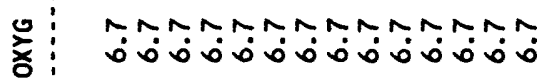
앰

胥突

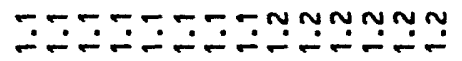

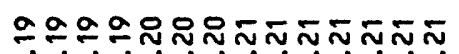

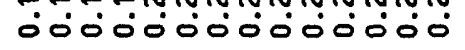

0.0000000000000

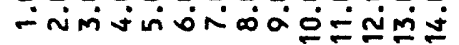

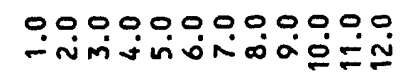

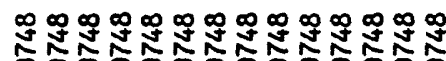
00000000

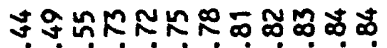

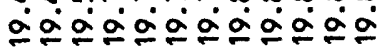

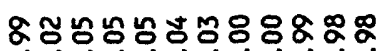

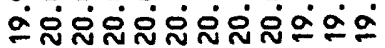

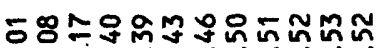

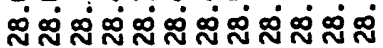

$\stackrel{\infty}{0}$

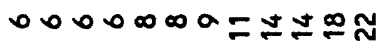

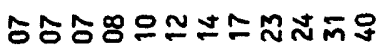
०0000000000

in

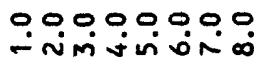

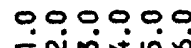

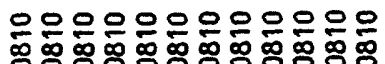

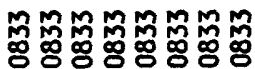

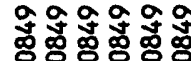

우우으우우읕ㅌ

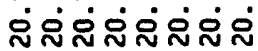

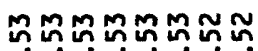

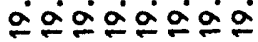

NกNNKNKN พิ่

$\stackrel{0}{0}$

$000 \infty 000=$

$M M \simeq \simeq \simeq M \pm ニ$

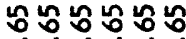

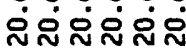

๓๐ఃะః $\stackrel{\infty}{\infty} \dot{\infty} \propto \omega$

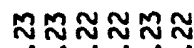

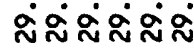

$\ddot{\circ}$

$\alpha \infty \infty \infty \infty$

$\simeq \simeq \simeq \simeq \cong=$ 000000

$\stackrel{0}{a}$

\section{: $8: \mathbb{8} ロ \mathbb{8}: \mathbb{8}$}

$\infty \infty \infty \infty \infty \infty$ ט.

0.9090
0.0900

$$
\stackrel{0}{0}
$$

0.9 .9 .9 .90

으는ํㄴ응 00000

$$
\text { ஸீ. }
$$

$\stackrel{9}{-}$

\section{-nmuino}

000000000000

0,0000000

000000

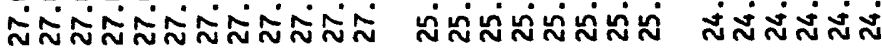


กุ

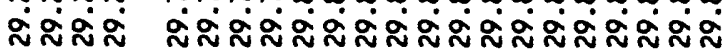

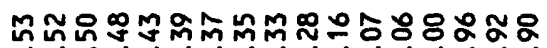
$\infty \infty \infty \infty \infty \infty \infty \infty \infty \infty \infty \infty$ $\stackrel{9}{0}$

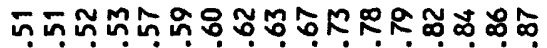

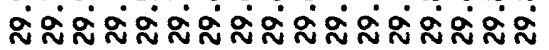
a

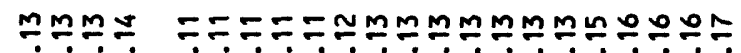
$\ddot{\circ}$ :000

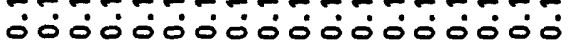

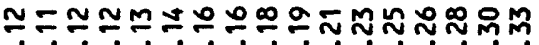
व000000000000000

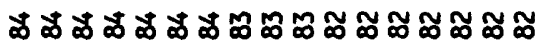

\$ 000000000000000000000

00000000nunnnununn Gaga MNMNMNMNMNNONNMNON

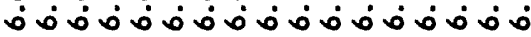

0000000000007.7 .7 .7

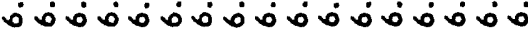

$$
\ddot{0}
$$

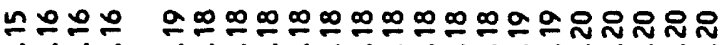

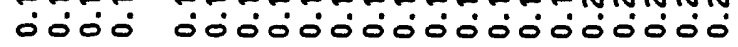

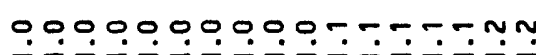

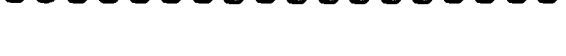

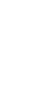

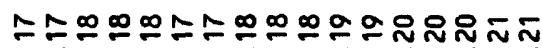

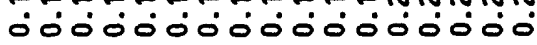

$\overline{0}$

00000000000000000

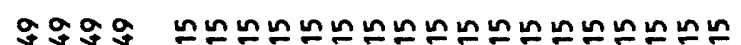

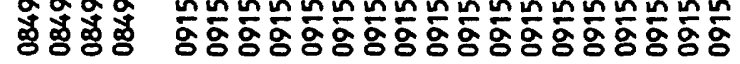

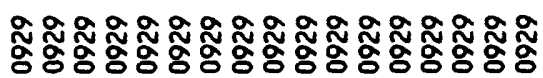




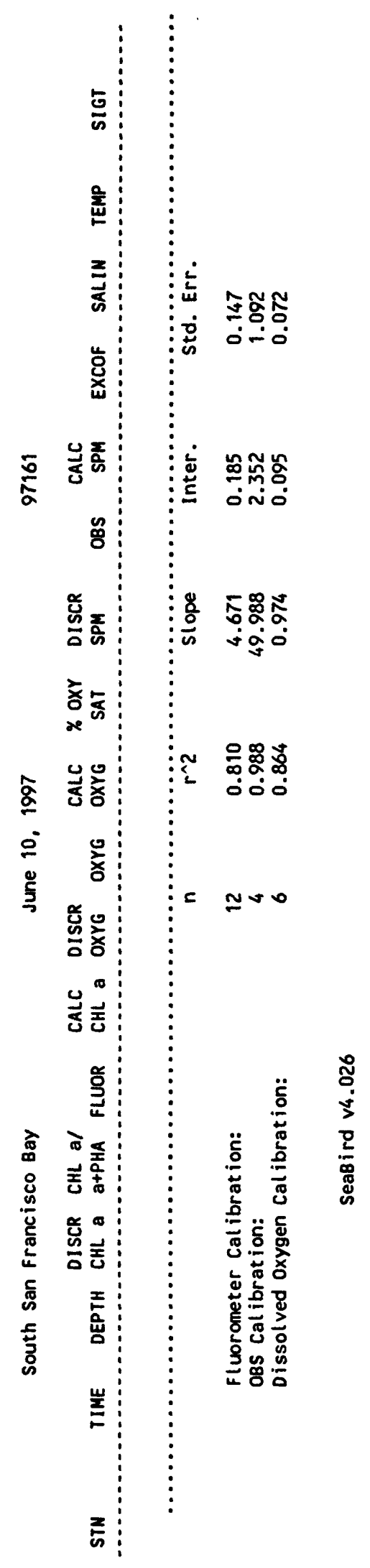


888888888888 88888088880

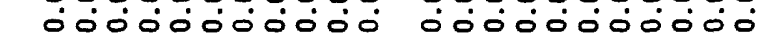

\section{ำำะ}

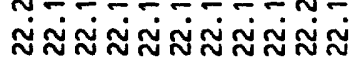

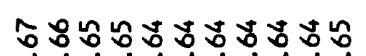
ะะะะะะะะะะ ஸं00ं00000. $\bar{i}$

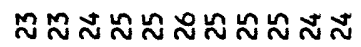

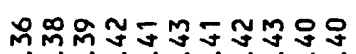
0ं00000000

$\stackrel{\sim}{\sim}$

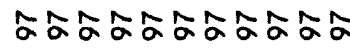

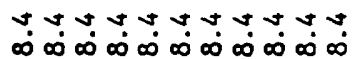

ชิ

$\stackrel{n}{2}$

$\frac{2}{3}$

芯 음

롫

0.000 .000000

intivinition

m.

0.0 .0 .0 .0 .000 .000

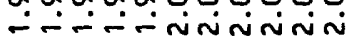

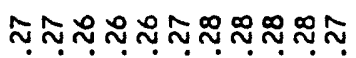

O0000000000

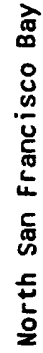

$\check{n}$

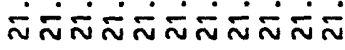

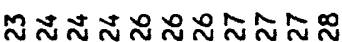

0ं00000000

$\stackrel{\circ}{m}$

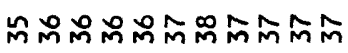

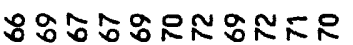

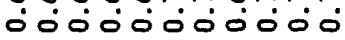

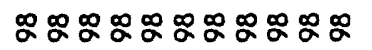

00000000000 $\infty \dot{\infty} \infty \dot{\infty} \infty \dot{\infty} \dot{\infty} \infty \dot{\infty} \infty \dot{\infty}$

$n-n \sim n \sim m \sim n n$ $\infty \infty_{\infty}^{\infty} \infty \infty_{\infty}^{\infty} \infty \dot{\infty} \infty \dot{\infty} \infty \dot{\infty}$

$$
\stackrel{\circ}{\infty}
$$

$\min 0 \wedge N \wedge N \infty \infty a$ iñnininininin

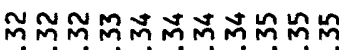

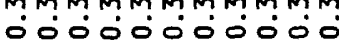

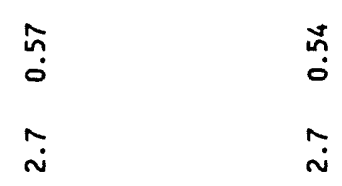

00000000000

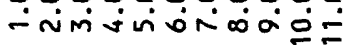

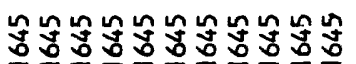

00000000000

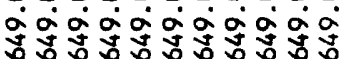

กั

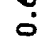

$\infty$

0.0000000000.

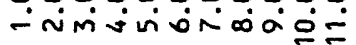

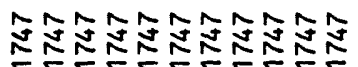

00000000000

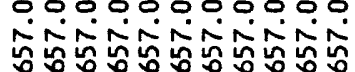

88888080808080

ல் $\dot{0} 0 \dot{0} 0 \dot{0} 0 \dot{0} 0 \dot{0}$

888888

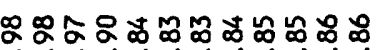

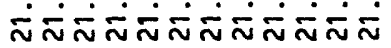

ธธธธปังดำำกัก

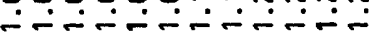

$\stackrel{\circ}{\dot{m}}$

$\stackrel{\sim}{i}$

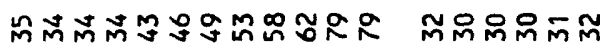

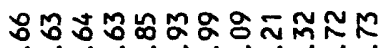

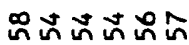

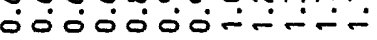
0ं00ं0

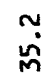

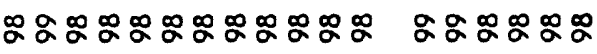

un un un un un un un un un m

แก แก แก แ แ

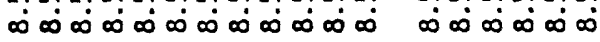

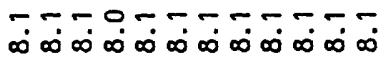

$---000$

$$
\stackrel{\infty}{\infty}
$$

devannm

oo 0000 viñinininimim inin- -

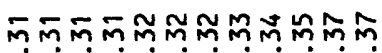

พิกลกลกลิ 00000000000 000000

$$
\stackrel{0}{\circ}
$$$$
\dddot{\sim}
$$

000000000000

0.000 .00

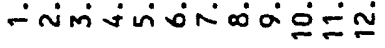
- imisio

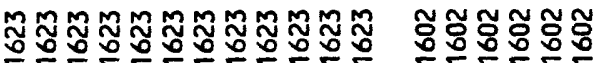

0,000000000000000000

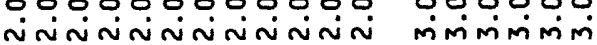




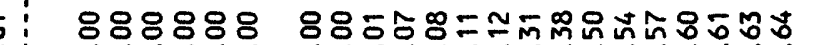

000000 00000000000000

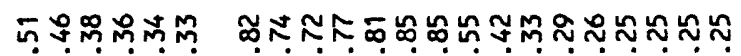

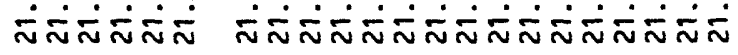

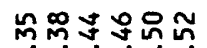

iñiññ

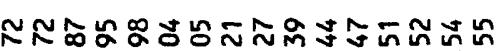

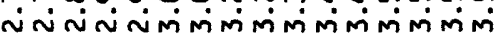

$\stackrel{\sim}{\sim}$

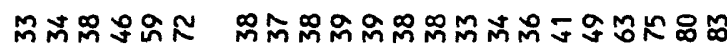

ำกณกำ

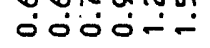

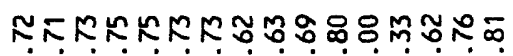

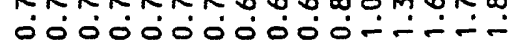

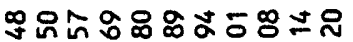

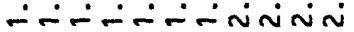

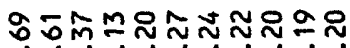

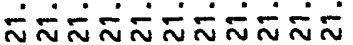

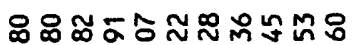

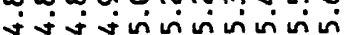

$\stackrel{\infty}{\sim}$

$\tilde{\sim}$

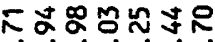

mimisio is

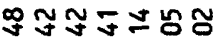

$\bar{\sim} \bar{\sim} \bar{n} \bar{n} \bar{n} \bar{N}$

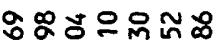

$\sim \sim \sim \infty \infty \infty \infty \infty$

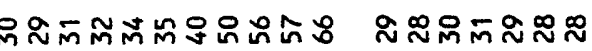

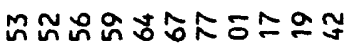

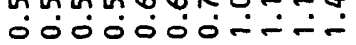

的夺识识品多早 0000000

\section{$\stackrel{\sim}{m}$}

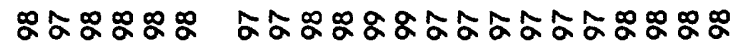

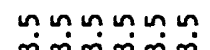

m. $\left.\infty \infty_{\infty}^{\infty} \infty \infty \infty \infty \infty \infty \infty \infty \infty \infty \infty \infty \infty \infty \infty \infty \infty \infty\right)$

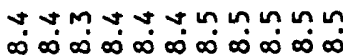

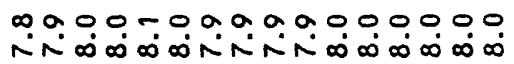

000.000

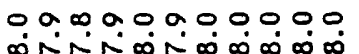

NomNnN

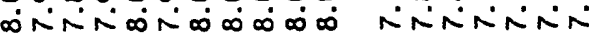

\section{$\stackrel{m}{\infty}$}

0.90 .90 ?

NNOONDa000

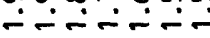

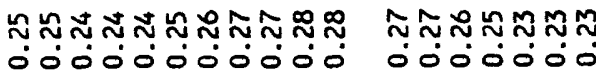

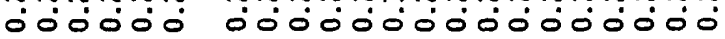

웅

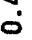

$\stackrel{\circ}{\sim}$

윰몽

0000000000000000000000

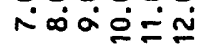

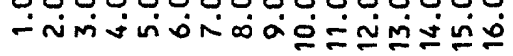

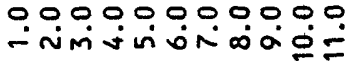

$\overline{5}$

$\stackrel{m}{-}$

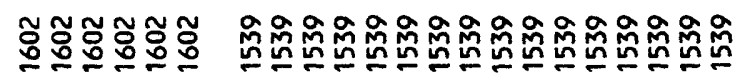

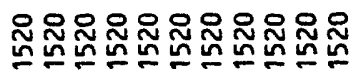

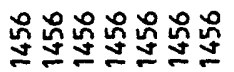

0000000 


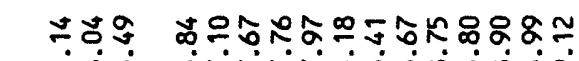

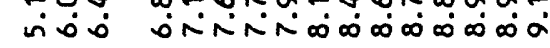

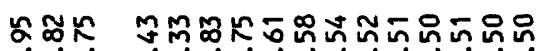

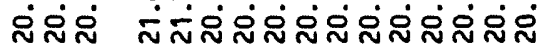

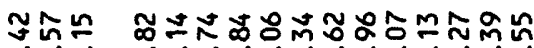

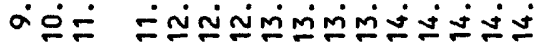
$\stackrel{\sim}{\sim}$

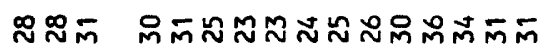

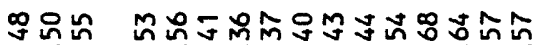
000 00000000000

음

爻邱

$\frac{5}{2}$

정징

ก

음

选논

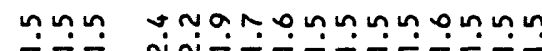

nn-.-.-.-.

ก

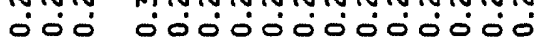

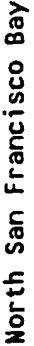

옳

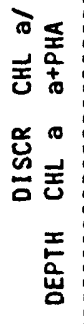

訔

0.000000000000000

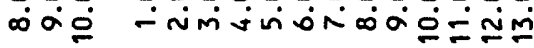

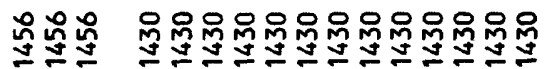

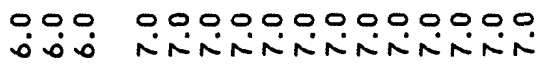

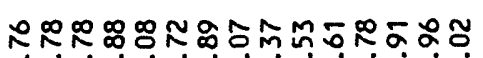

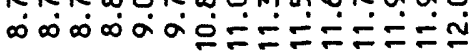

๒

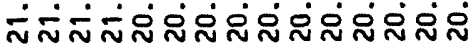

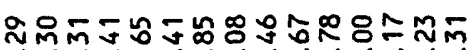

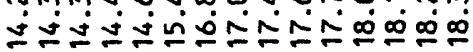

$\bar{n}$

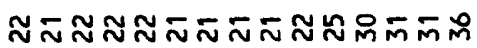

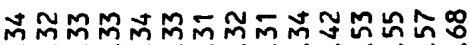
0ं0000000000000
ํํำำำํำำ

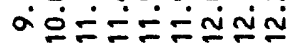

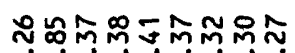

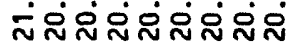

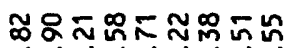

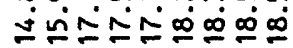

$\bar{i}$

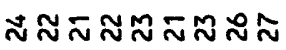

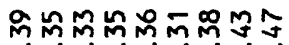
o.0000000

พั่

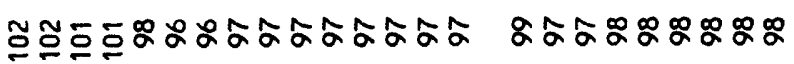

mmmnowagaaaaaaa क्ष

DoNNMNMmMMmMmMm

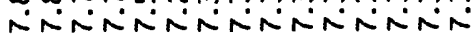

momanmmmom $\bar{\infty}$

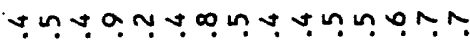

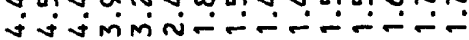

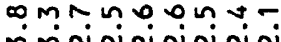

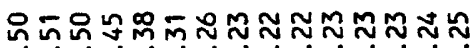

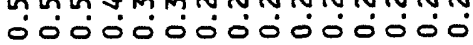

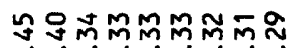

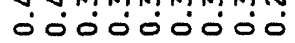

욤

$\dot{\circ}$

$\dot{5}$

0.0000000000000

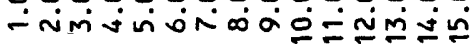

0.0000000

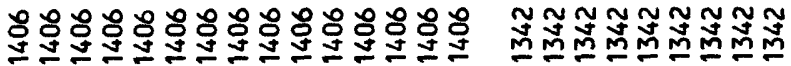

000000000000000

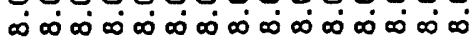

000000000 áåáåá 


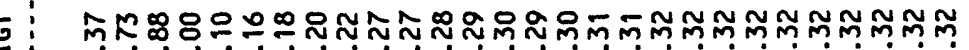

s

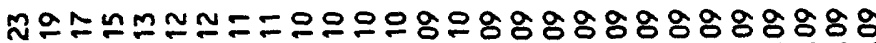

釭

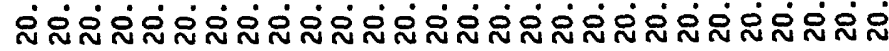

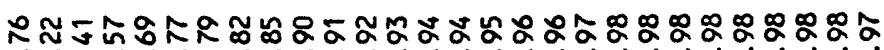

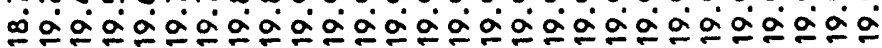

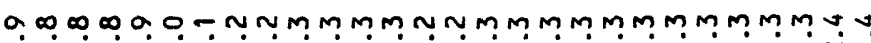
-

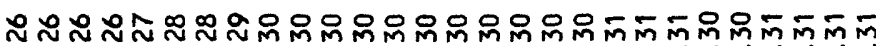

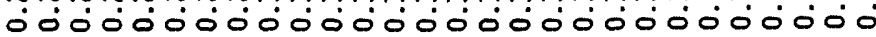

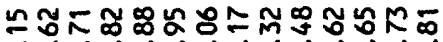

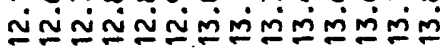

-

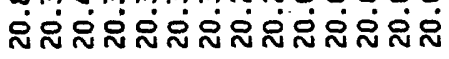

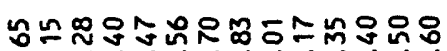

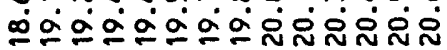

$\stackrel{9}{\circ}$

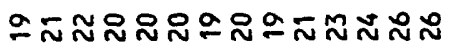

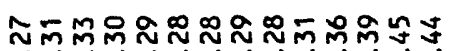
0ं00ं0்0000000

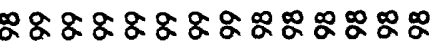

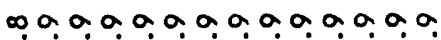

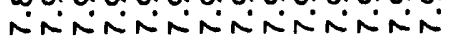

nmm

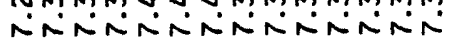

$a$ andornammnmimininimiñiñinin

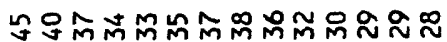
0000000000000
0000000000000000000000000000

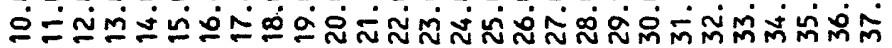

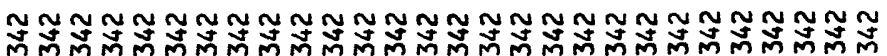

崖

2
0000000000000000000000000000

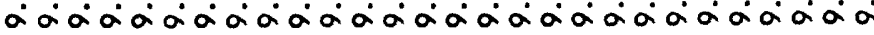

00000000000000 -

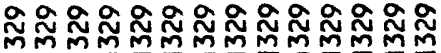

00000000000000 응ㅇㅇㅇㅇㅇㅇㅇㅇㅇㅇㅇㅇ 


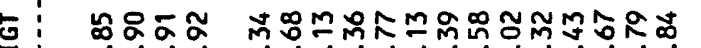

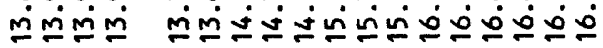

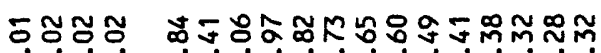

穴品

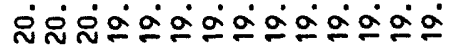

ถึกN

กิํํำ

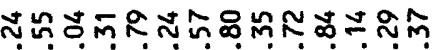

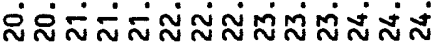

$\stackrel{\infty}{-}$

๗̊ำ

ㄱำํํㅇำ

०००0

递

爻產

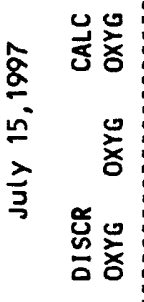

离
똥론

总

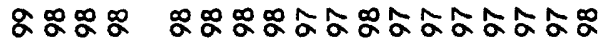

0000

NNN

$m m m m$ NNN

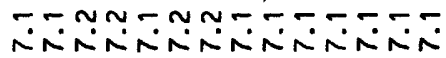

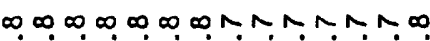

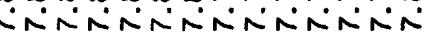

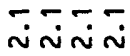

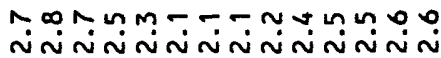

$\stackrel{\infty}{\infty} \stackrel{\infty}{\sim} \underset{0}{\sim}$

O०००

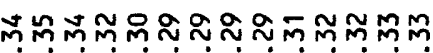

0000000000000

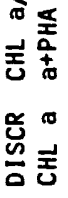

点

0.0 .0

ํํํ요

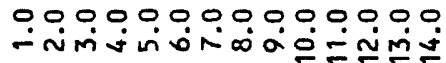

ำํํำำำ

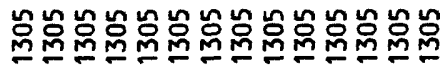

z:

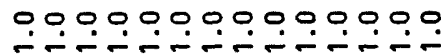

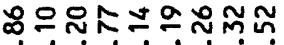

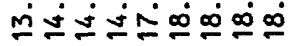

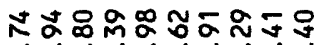

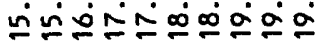

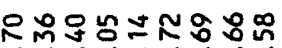

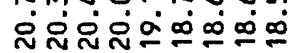

ㄸڤํํำำำ유

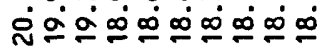

ஐ

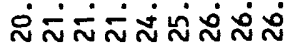

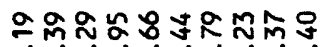

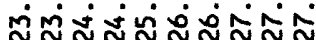

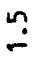

$\dddot{-}$

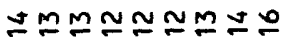

웅aaa으으으므

$\infty \infty$

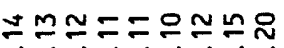
00000000

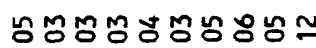
0000000000

5웅

$\stackrel{0}{\circ}$

๕ญํำละะัล

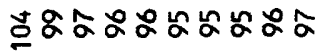

ะั

$m \checkmark n N \backsim \infty R N$ $\infty \infty \infty N N R N$

N orananararar

monoaOoOñNañNan

NNO000000NANRODONR

क.

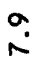

on m mon 00 åñniñ

M ONUO OUSMM mumiñ

in

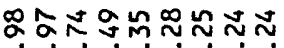

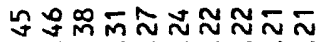
0000000000

$\stackrel{\infty}{\sim}$

চ.

กั่

$\hat{m}$

$\dddot{-}$

0.00000000

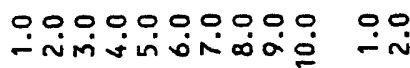
- Nimivioña

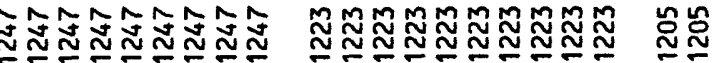

0,00000000

0000000000

0.0

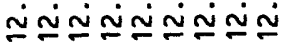

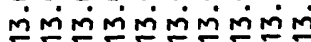

ปั 


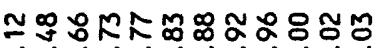

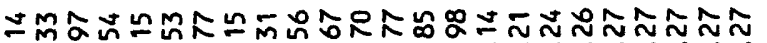

ํㅜㄴํํำ

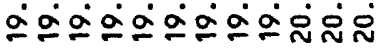

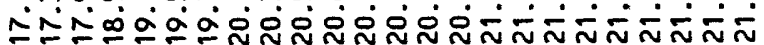

ํํ묘

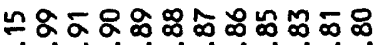

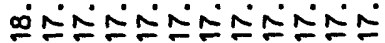

느

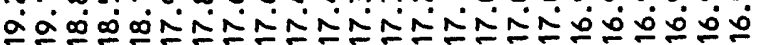

凤下范出

$\ddot{\circ} \div \div \div 0$

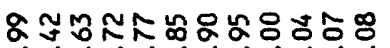

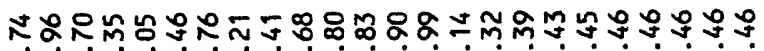

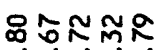

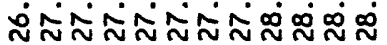

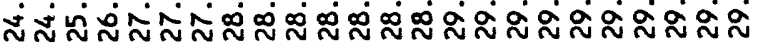

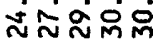

$\dddot{\sim}$

0

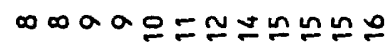

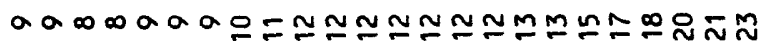

$\infty \infty \infty \infty \infty$

ธธธ์ํํำํํㅇำำำ

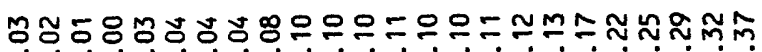

ó0ं0ं0்

88888

00ं00

an

过

ชัญ

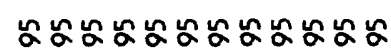

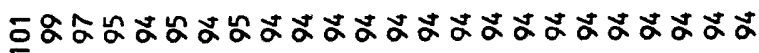

ลัฐณณณ

苗

$\infty$

Oด

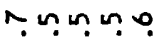
a

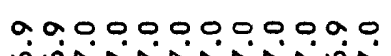

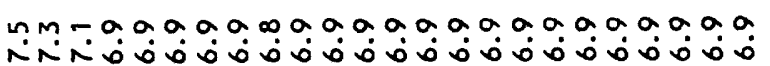

$\because 9009$

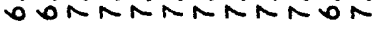

$$
\stackrel{a}{\sim}
$$

过

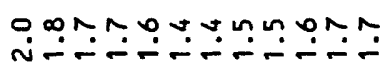

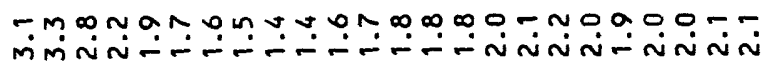

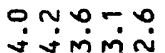

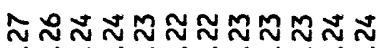
00000000000.

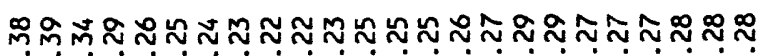
ó00000000

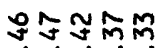
ז

0.000000000000

000000000000000000000000 $\therefore$ ก

00000

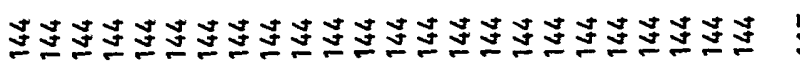

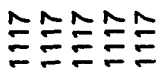

0.000 .0000000

000000000000000000000000

00000 点

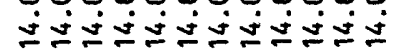

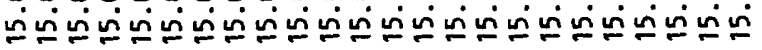
ํํㅇㅇㅛ 


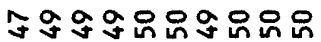

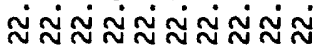

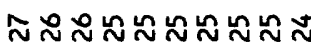

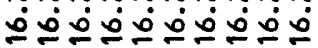

ఐ

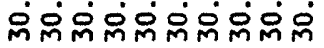

蒙

농

$\stackrel{2}{a}$

$\infty \infty \infty \infty \infty \infty \infty \infty \infty$

8888888888

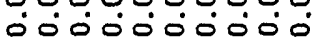

递

ชัญ

$\frac{2}{3}$

렁 징

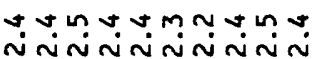

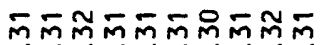
000000000

\section{min}

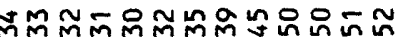
ó000000000

88888888884

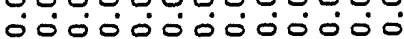

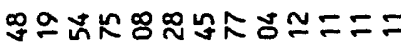

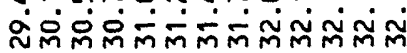

$\stackrel{\infty}{0}$

$\infty \infty \infty \infty \infty \infty \infty \infty \infty \infty$ 으으

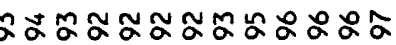

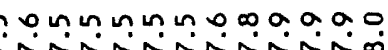

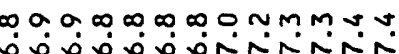

Nonaminonastor iñNiñimmsso 总

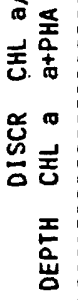

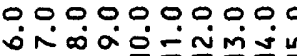

0000000000000

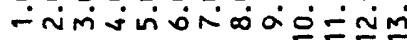

\section{ミミミミミミミミミミ

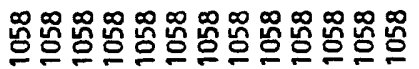

崖

0000000000

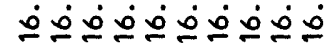

0000000000000

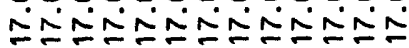

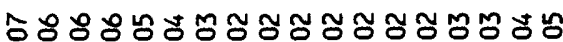
0000000000000000

$\stackrel{0}{j}$

- 90.00000000000000

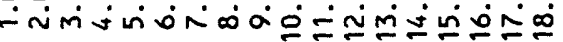

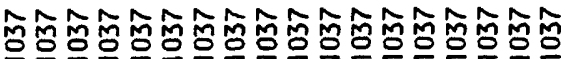

กับ

$\hat{\sim}$

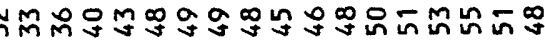
0000000000000000

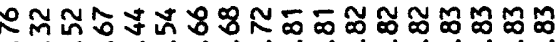

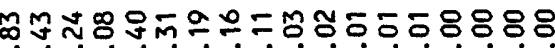

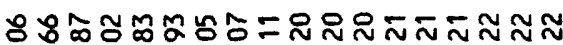

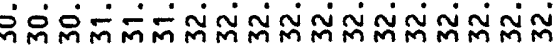
$\hat{o}$

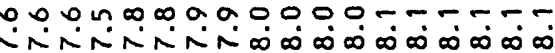

a a a $a$ - umm $\stackrel{i}{i}$

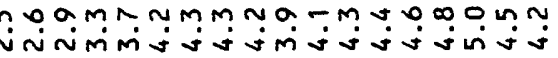

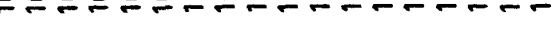

000000000000000000

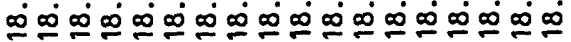




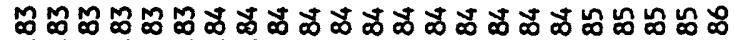

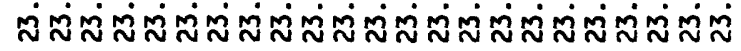

$8888888888888882 \% 2 \% 2 \%$

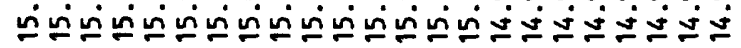

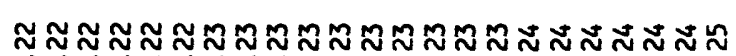

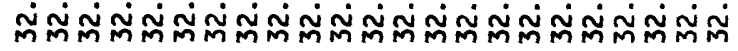

으으으으우으우우우으으으으으으으 $000000 \infty$

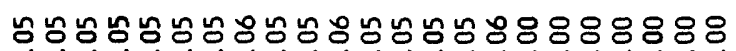

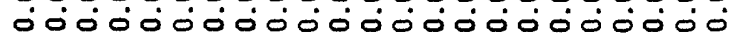

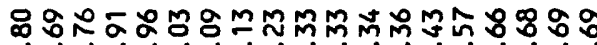

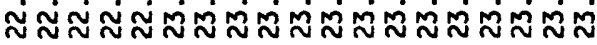

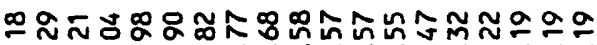

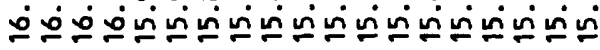

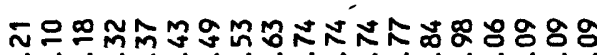

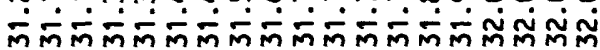
$\stackrel{0}{\circ}$

ㅇaa.00000으읕등ㅇㅇㅡ

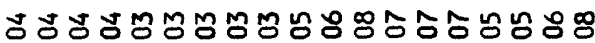

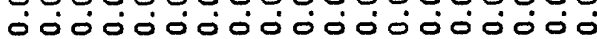

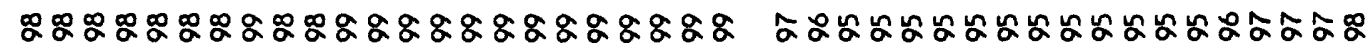

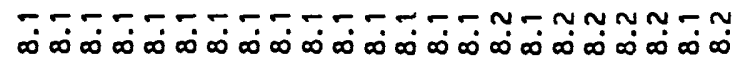

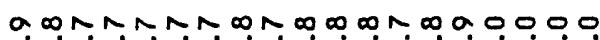

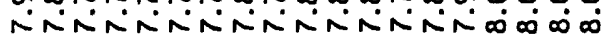

?

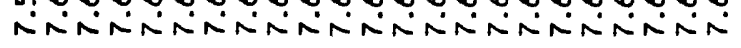

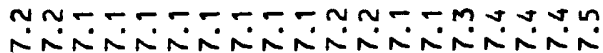

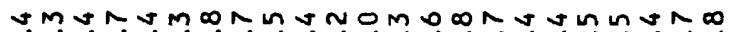

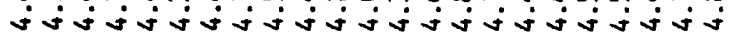

NNa-ror-aONNNMUDO0

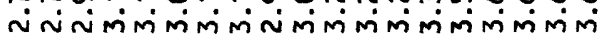

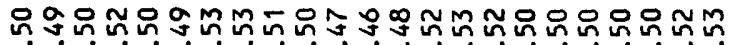
00000000000000000000000

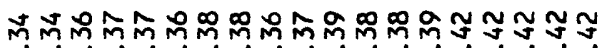
0000000000000000000

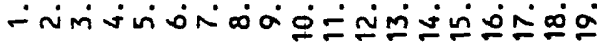

$\infty \infty \infty \infty \infty \infty \infty \infty \infty \infty \infty \infty \infty \infty$

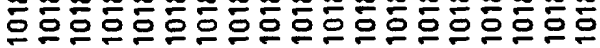




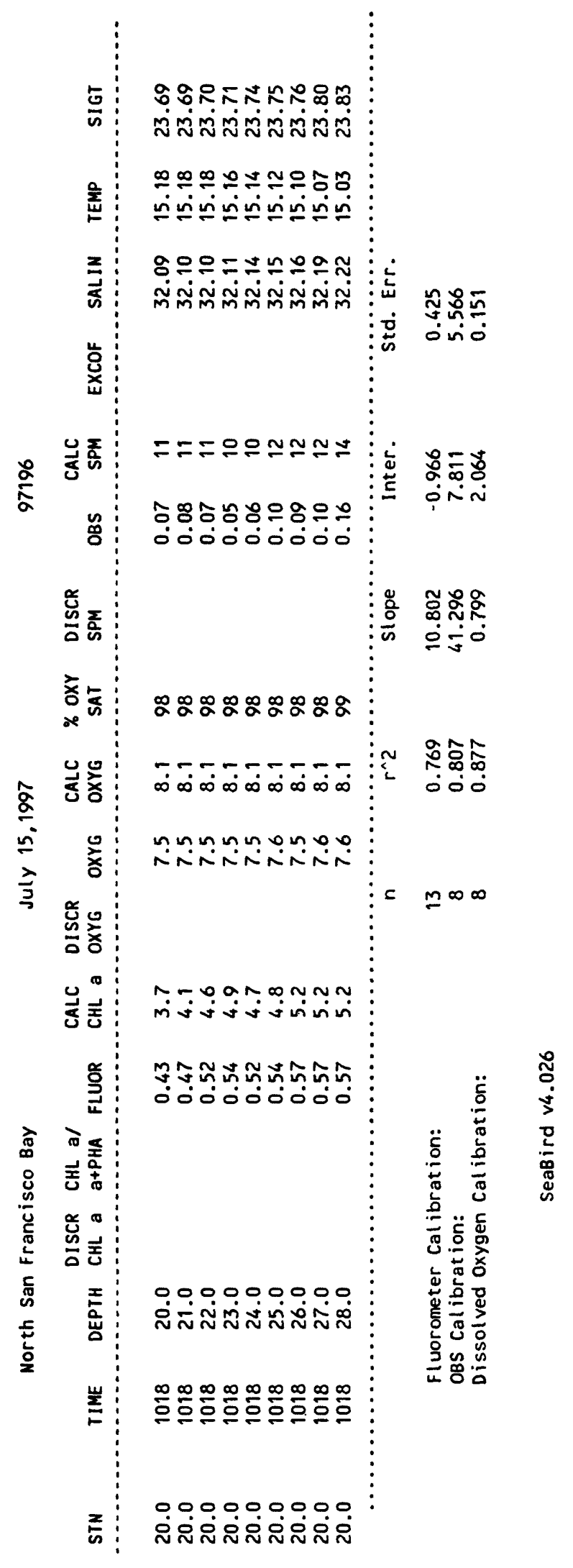




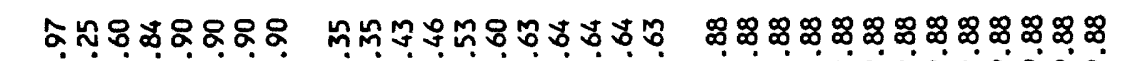

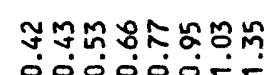

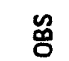

总

ชัญ

ڤั

n்

$\frac{2}{3}$

종

음

迅롱

s

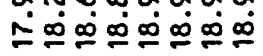

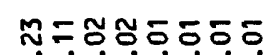

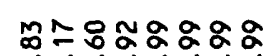

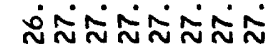

$\stackrel{-}{-}$

๗๗๊̄

ฌ்

高

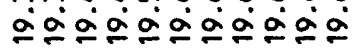

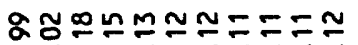

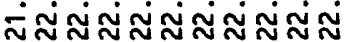

ที่์ก์

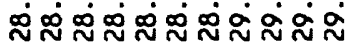

$\stackrel{m}{ }$

ニニニニニッㅇN요

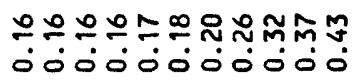

$\stackrel{+}{a}$

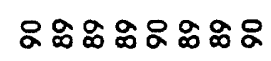

每

r

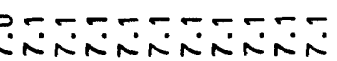

กั0.0.0.0.

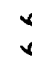

ำ

9

nกำ

mंñmisimimi

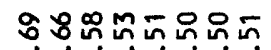

00000000

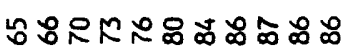

ல0ं0000000

$n$
0
0

ঙุ.

$\overline{0}$

in

j.

$\tilde{m}$

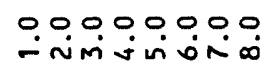

崖

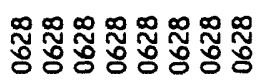

00000000

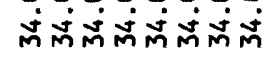

$a$

00000000000
0000000000000

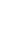

o.

i

$\infty$

000000000000000000000000

-nimiviơ

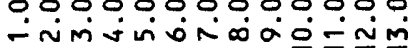

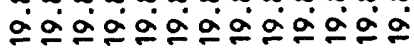

영영ㅇㅇㅇㅇㅇㅇㅇㅇㅇㅇ으으

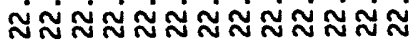

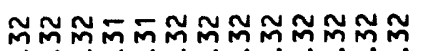

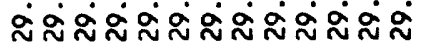

$\stackrel{m}{?}$

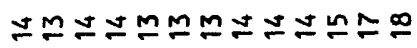

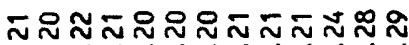

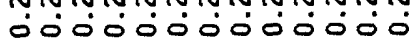

$\stackrel{m}{\sim}$

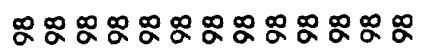

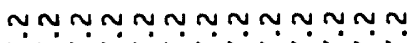

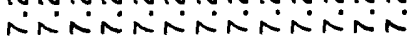

9.999.99.9.9.99.9.9.

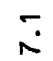
iñiñinimminmmin

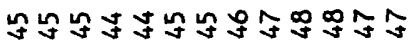
000000000000

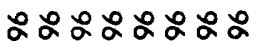

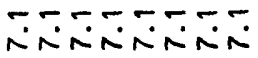

N $\infty \infty \infty \infty \infty \infty$ من

$\infty \infty \infty \infty \infty \infty \infty$

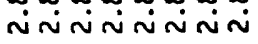

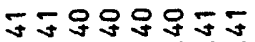
0000000

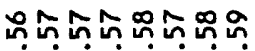
$\bar{n} \bar{n} \bar{N} \bar{N} \bar{N}$

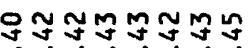

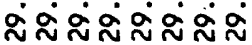

$\stackrel{9}{-}$

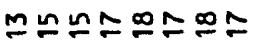
व0ं0000

오으으으으는

00000000 - Nimvivió

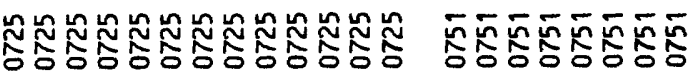

0000000000000

0000000.

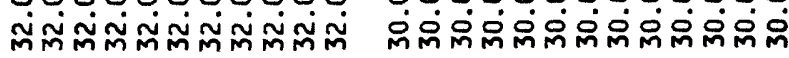

ลำลำลำ 


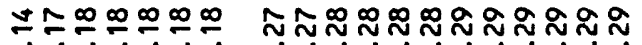

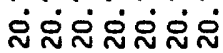

бмตำตำ

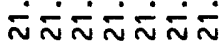

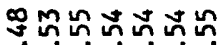

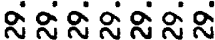

\section{$\stackrel{\circ}{-}$}

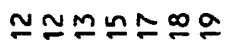

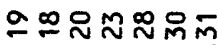

वं00000

总突

文空

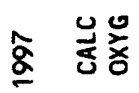

$\stackrel{0}{\lambda}$

3

음

选至

ma.0.0.0.

ininiminim

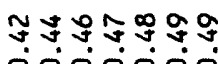

ப்0ல்

命

-

$\infty \infty a \sigma \infty \infty \infty$ $\dot{0} \dot{0} \dot{0} \dot{0} \dot{0} \dot{0} \dot{0}$

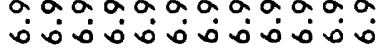

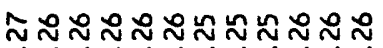

玄市六六六六市市市

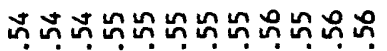

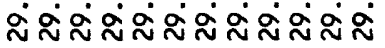

a0a0으므느유

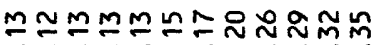

00000000000

$\overline{0}$

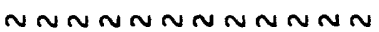

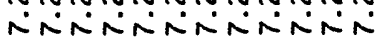

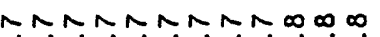

iñiniñininin

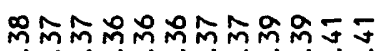

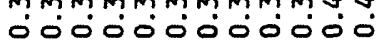

กั

กั.

$\bar{i}$

$\stackrel{\sim}{\sim}$

0.000 .00

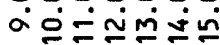

0.0000000000

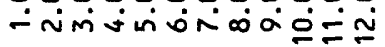

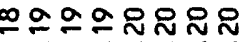

玄六六六六市

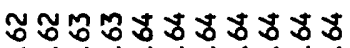

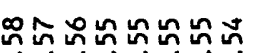

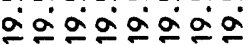

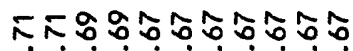

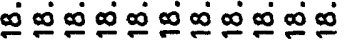

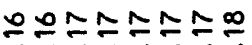

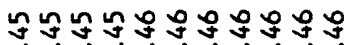

نिं

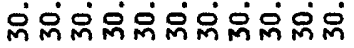

$\dddot{0}$

0

$\infty \sim \infty \infty \infty \infty \infty \infty$

$\infty \infty \infty \infty \infty \infty \infty \infty \alpha$ ?

으영으읃ニ

ல0ं0்000

으으으으읖ニㅍ வ்

$\hat{\infty}$

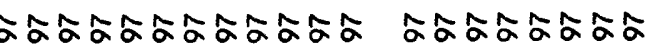

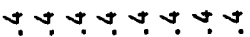

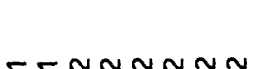

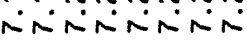

$\infty \infty \infty \infty \infty \infty \infty \infty \infty \infty \infty$ $\dot{0} \dot{0} \dot{0} \dot{0} \dot{0} \dot{0} \dot{0} \dot{0} \dot{0} \dot{0} \dot{0}$

$\check{n}$

บ N $บ$ Mก

miniminiminim

ח iññññññ

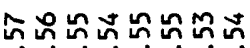
ธั0்

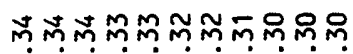

0.000000 .0 ○ं0ல் 000000

$\stackrel{8}{\circ}$

ก̊:

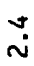

m

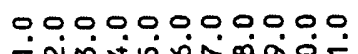

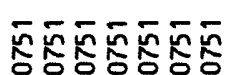

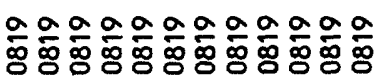

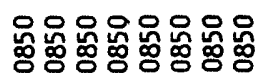

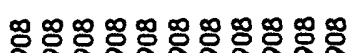

荘

00000000

0.000 .000000$.

00000000

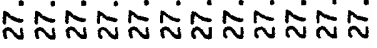

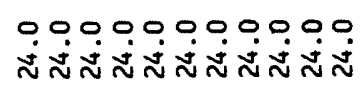




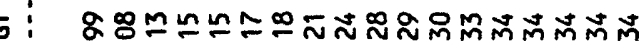

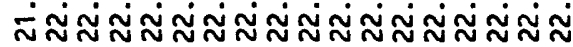

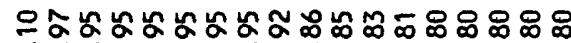

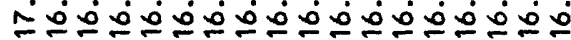

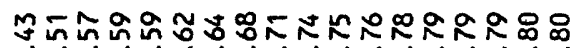

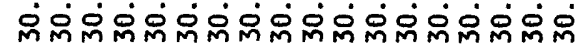
$\stackrel{\infty}{\circ}$

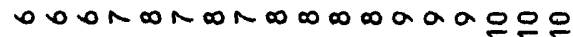

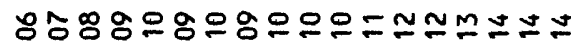
000000000000000

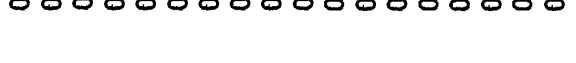

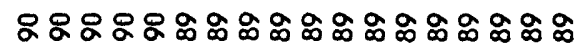

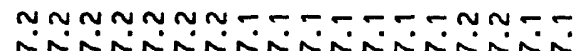

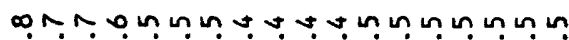

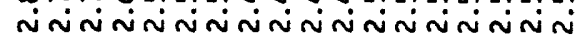

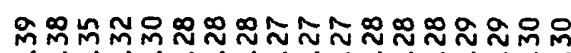
0000000000000000

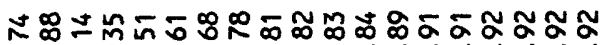

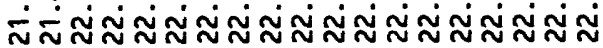

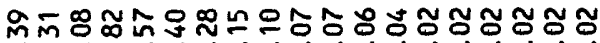

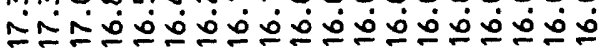

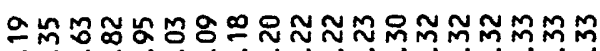

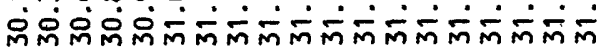

$\stackrel{9}{0}$

0 0 on $\infty \sim \wedge \sim \sim \infty \infty \infty \infty \infty \infty \infty$

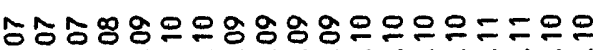

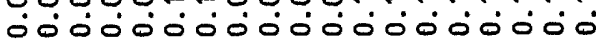

$\stackrel{0}{\circ}$

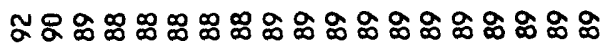

m?

- 0 -

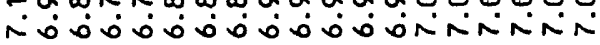

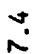

ด.

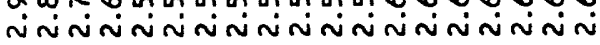

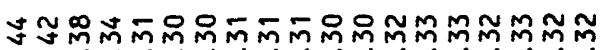
000000000000000000

?

$\stackrel{m}{n}$

$\dot{m}$

$\stackrel{a}{i}$

000000000000000000

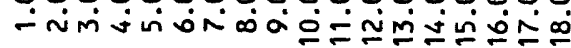

0000000000000000000 -

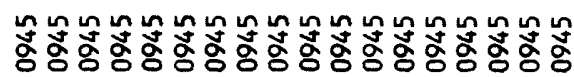

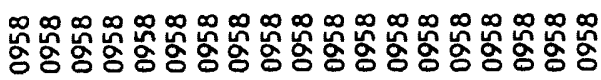

000000000000000000

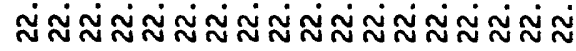

0000000000000000000

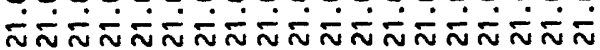


กิบ

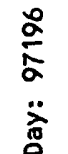

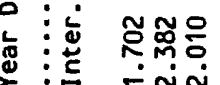

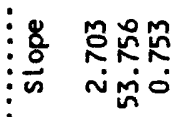

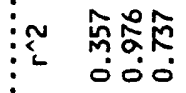

g

눙

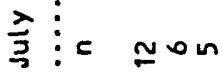

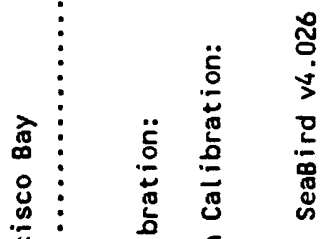

芯范

등

岕焉

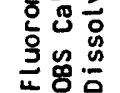


8888888888888888888888888888888

0ं00ं00000

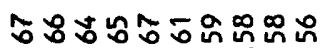

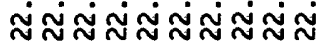

ํํㅇํํํํㅇํㅇํํํํㅇํㅇํำ

0ं0000000

i

$\frac{\nwarrow}{\alpha}$

达

\%

苟

ฟิธ

ล

in

管

웛 잉

봃론

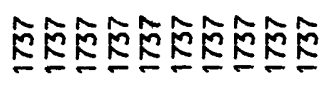

0.000000000

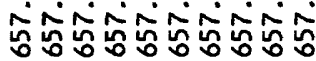

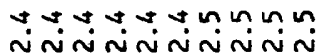

$\bar{m} \bar{m} \bar{m} \bar{m} \bar{m} \bar{m} \bar{m} \bar{m} \tilde{m}$

000000000

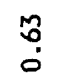

in

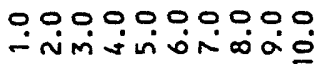

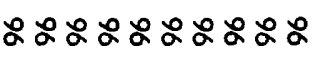

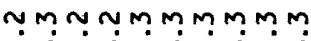

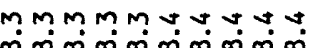

$\stackrel{\infty}{\infty}$

$\infty$

nninor

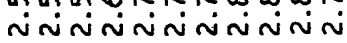

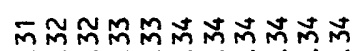
०0000000000

$$
\stackrel{\circ}{\circ}
$$

$\stackrel{\infty}{i}$

00000000000

- imivion

$\hat{n}$
MMMMMMMMM $\infty \infty_{\infty}^{\infty} \infty \dot{\infty} \infty \dot{\infty} \infty \dot{\infty} \infty$

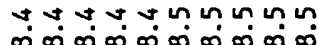

mingunin o o o o

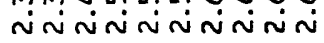

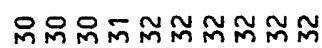
000000000

:용

0000000000 -

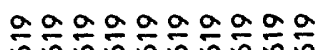

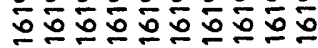

0000000000

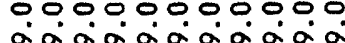

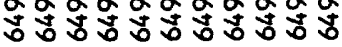

niñiniñiniñ
ஸ00000000

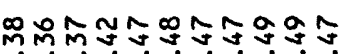

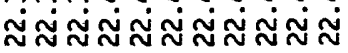

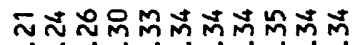
0000000000

$\dot{\sim}$

$\hat{m}$

NRETKRㅇ

0000000000

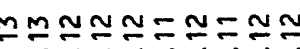

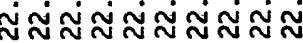

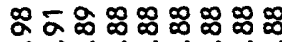

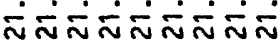

พัธธธธธธธธธอ

10.60 .50

j
888888888 00000000

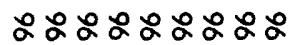

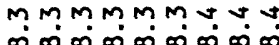

$\checkmark \ln v \ln u \operatorname{un} u \ln$ $\infty \infty \infty \infty \infty \infty \infty \infty \infty \infty$

$$
\stackrel{+}{\infty}
$$

mmotun un un un iññññó

品品的可可的的管 वं०0ं00ं0

:

$\stackrel{\sim}{\sim}$

000000000

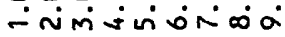

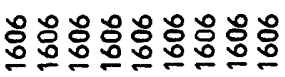

000000000

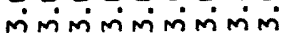




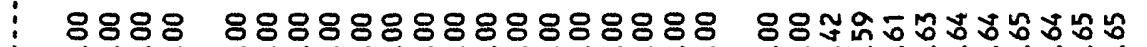

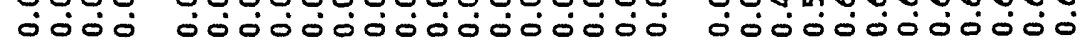

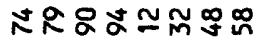
$\therefore-$-nininin

ஐ

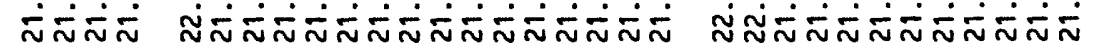

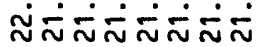

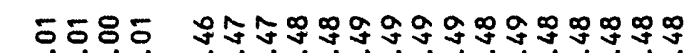

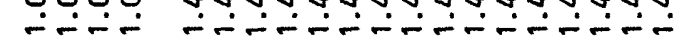

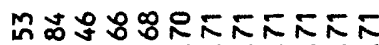
iñminiminiminiminim

సักำำㅇํㅇㅇㅛ ininiminisio $\hat{n}$ $\stackrel{0}{0}$

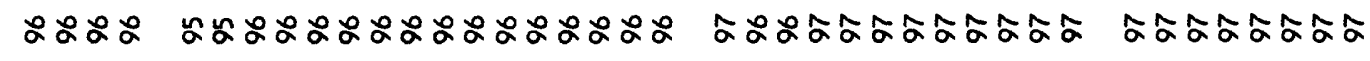

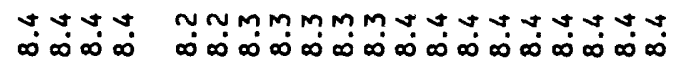
$\infty$

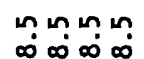

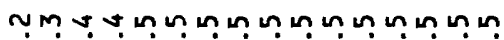
in

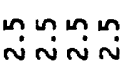

iñ

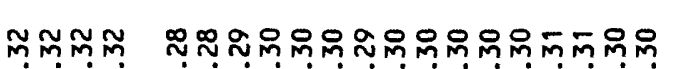

0000 0000000000000000

กิ?

i

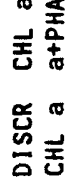

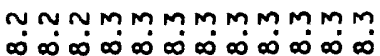

$m m m v d v d v m m u n$ $\infty \infty_{\infty}^{\infty} \infty \infty_{\infty}^{\infty} \infty \dot{\infty} \infty \dot{\infty}$

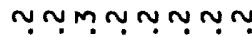
$\infty \infty_{\infty}^{\infty} \infty \dot{\infty} \infty \infty_{\infty}^{\infty} \dot{\infty}$

$m m \checkmark m m m m m$ $\infty \infty_{\infty}^{\infty} \infty \infty \infty \infty \infty \dot{\infty}$

$m$

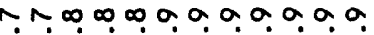

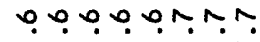

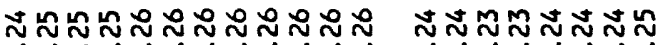

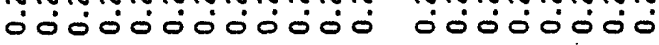

$$
\begin{aligned}
& 0 \\
& 0 \\
& 0 \\
& \hdashline
\end{aligned}
$$

000000000000

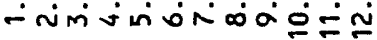

0.000000 -niminion

$M \underline{m} \underline{m} \underline{m} \underline{m} \underline{m}$ ํㅡำำำำำำำ
minimi

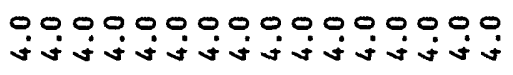
0.0000000000
00000000
00000000 


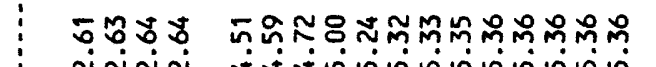

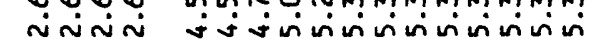

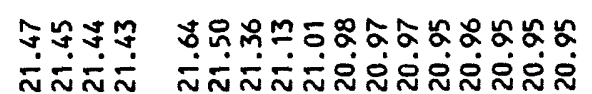

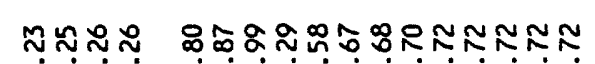

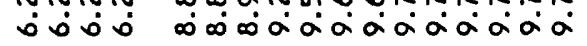

$\hat{\sim}$

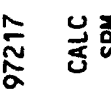

哭

递

ภัญ

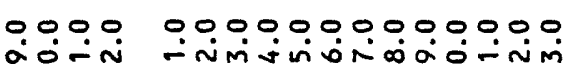

요요

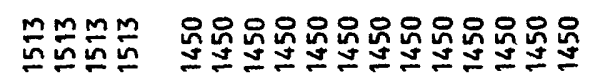

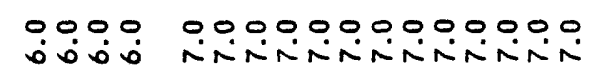

\section{กmmm 0.0000000000000.000}

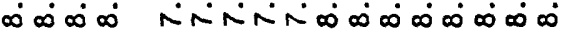

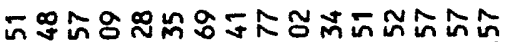
nivivio

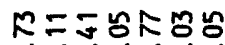

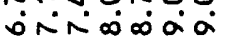

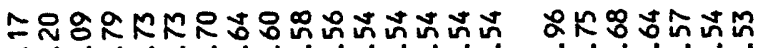

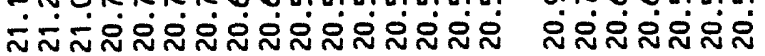

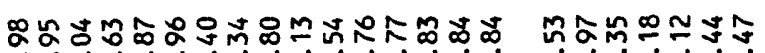

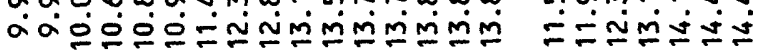
$\stackrel{\sim}{\sim}$

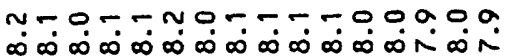

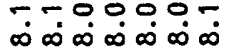

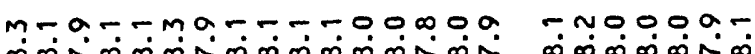
ம

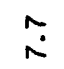

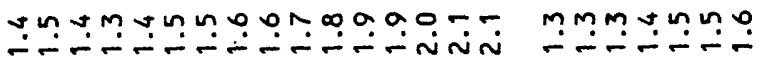

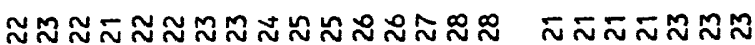
0000000000000000 0ं0ல00்<smiles>[CH-]1CO1</smiles>

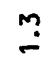

00000000000000000000000

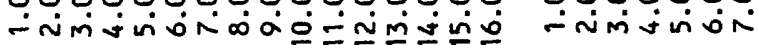

ம 


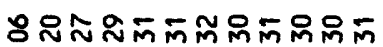

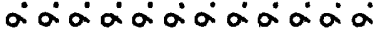

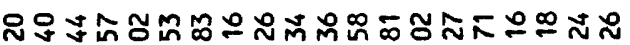

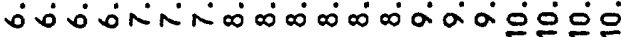

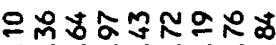

aरa $00 \div==$

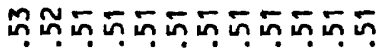

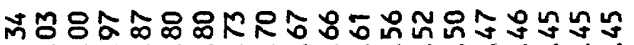

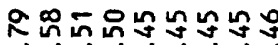

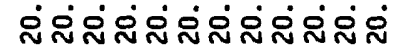

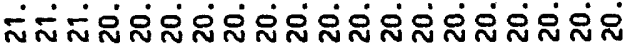

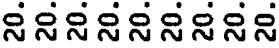

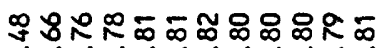

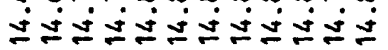

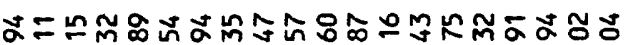

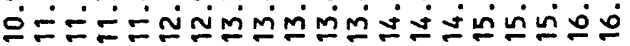

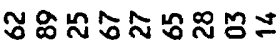

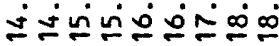

$\bar{i}$

$\stackrel{\sim}{\sim}$

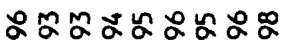

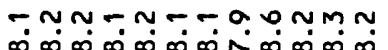

-

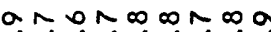

$-m \sim N M n-\infty \infty m n M$ $\infty \infty \infty \infty \infty \infty \infty \dot{\infty} \infty \infty \infty \infty \infty$

-ana-0- $+\infty \infty a a \sigma a \sigma a \infty \infty \infty \infty$ TnNaninin

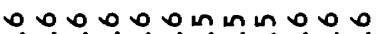

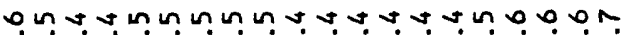

Rmunnn?mm

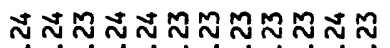

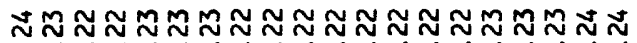

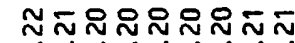

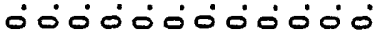

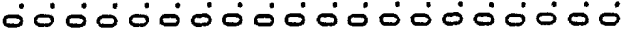

00000000

0.0000000000

00000000000000000000

000000000

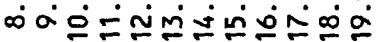

-ñंv

- imivinisio

崖

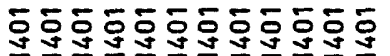

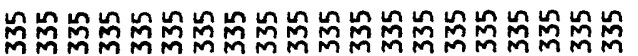

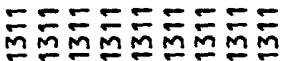




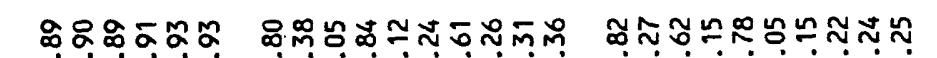

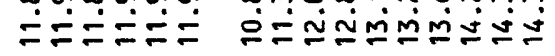

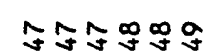

害

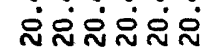

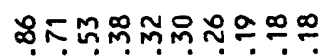

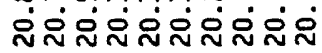

กุกฺฺฺฺฺ

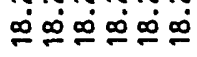

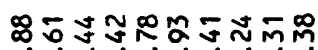

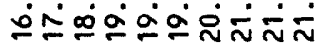

$\stackrel{0}{-}$

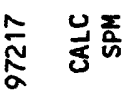

谷

魚

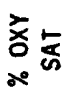

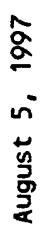

过安

ㅊ:

움

놇론
บับำฺฺ

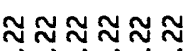

-0.000.

$\infty \infty \infty \infty 0$

ninitio

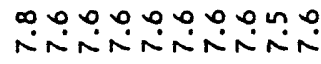

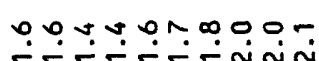

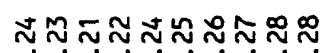

0000000000

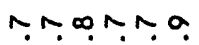

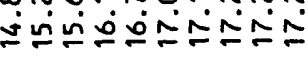

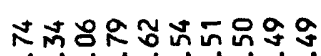

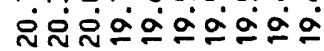

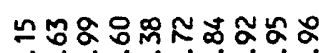

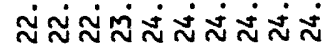
:

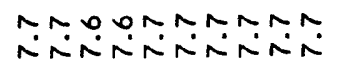

om tovm n. anaránar

$\tilde{n}$

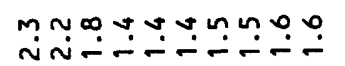

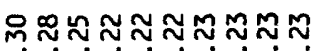
○்0ல்0ல0்0

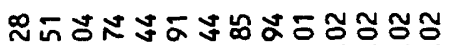

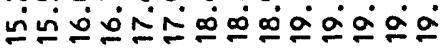

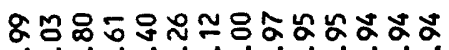

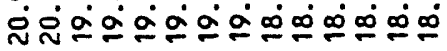

ฌ

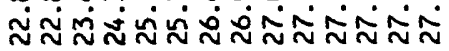

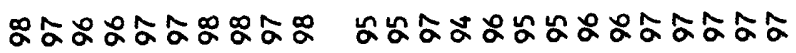

ง

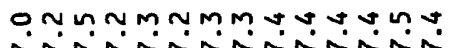

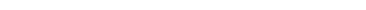

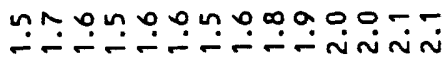

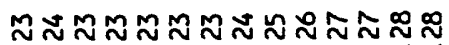<smiles>[CH]CO</smiles>

$\tilde{n}$

$\because$

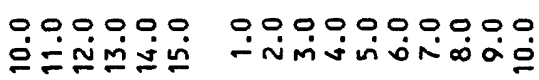

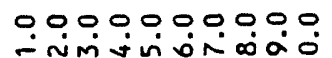

00000000000000 -

崖

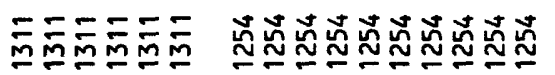

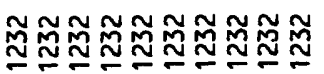

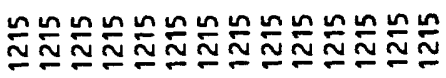

0000000000

0.0000000000000 0.000 .00000

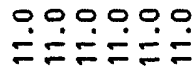

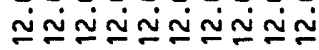

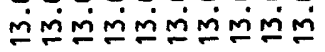

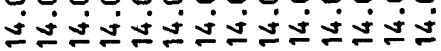




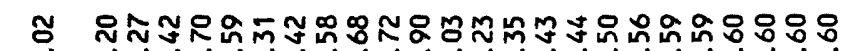

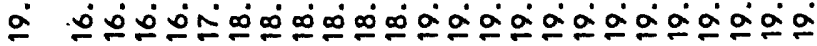

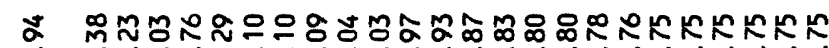

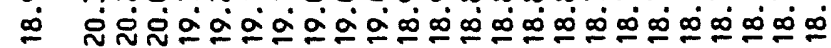

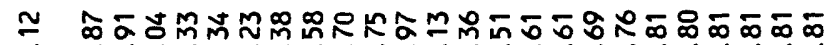

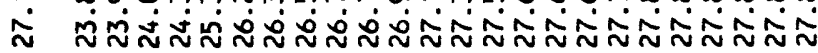

$\stackrel{\infty}{-}$

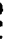

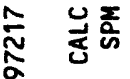

鱼

总

爻產

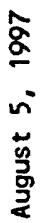

总

远突

בั:

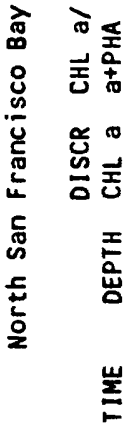

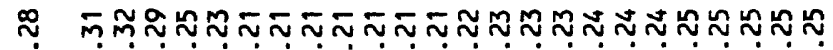

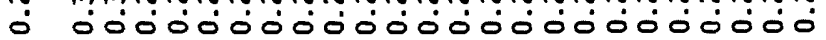

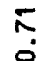

$\dot{0}$

$\stackrel{\infty}{-}$

$0 \quad 000000000000000000000000$

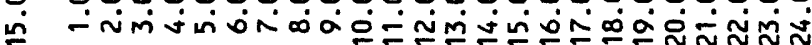

000000000000

- imivioniogí丶

웅ํํ

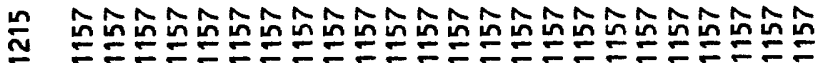

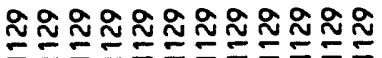

올을

0000000000000000000000000

孞 $\pm$

$\stackrel{n}{n}$

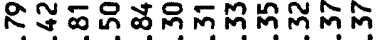

ละณ

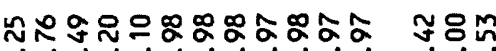

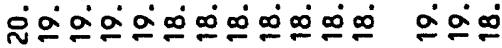

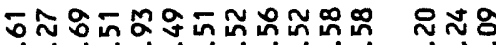

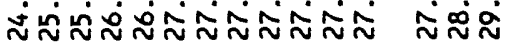

$\stackrel{\infty}{\circ}$

$\stackrel{\circ}{\sim}$

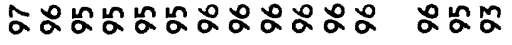

oñ

ñm

$m M \sim N \sim \sim N m m M m$

$\dddot{\Upsilon} \div 0$ NANANRNANA NŔ

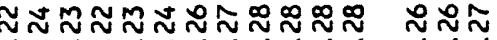
هั000000000 


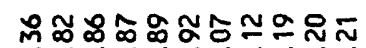

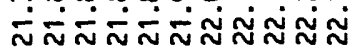

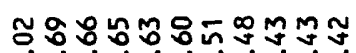

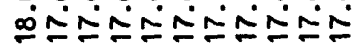

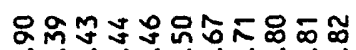

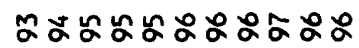

ty n m NRNRNRNRN

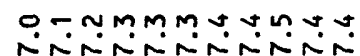

-M00月0.000\%inininininimimi

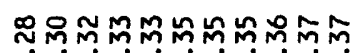
¿0000000000
00000000000

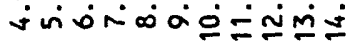

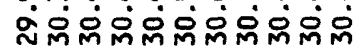

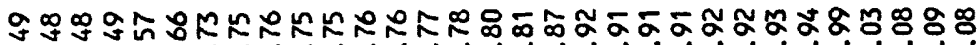

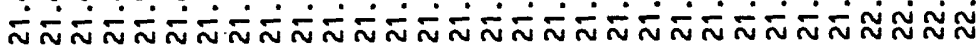

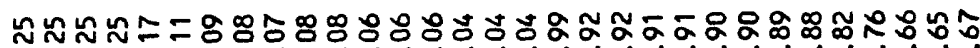

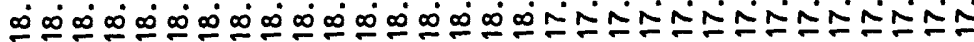

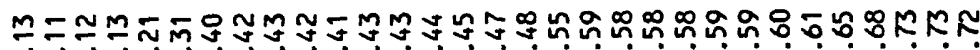

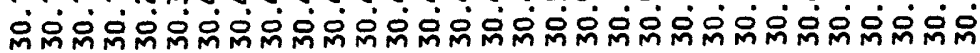
$\ddot{0}$

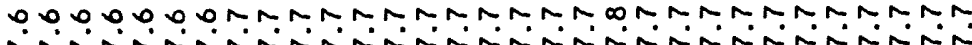

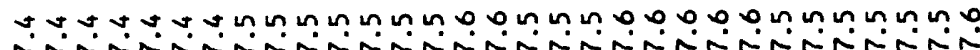
$\stackrel{n}{\sim}$

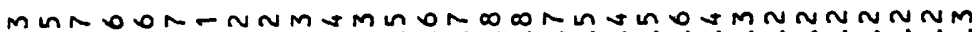

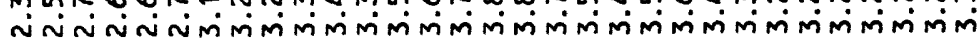

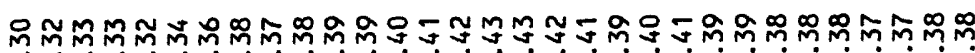
00000000000000000000000000
œ
(1)
$\tilde{m}$

0000000000000000000000000000000

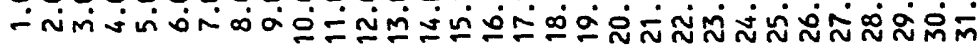

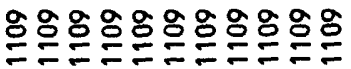

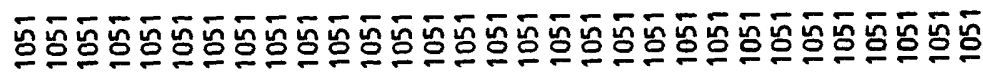

0.0000000000 กัง

0000000000000000000000000000000

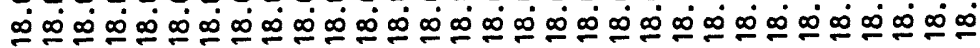


영용용으으으으으

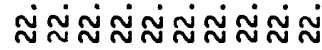

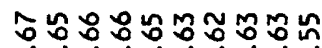

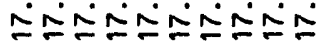

NKNRKNNNND

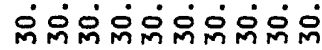

$\hat{\approx}$

玉 i

旁

通

岁

$$
\begin{aligned}
& \text { 军 }
\end{aligned}
$$

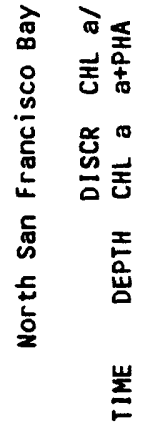

ImNMNMN-00 miniminiminimini

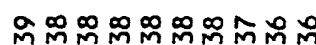
¿000000000
0000000000

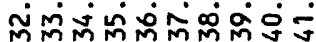

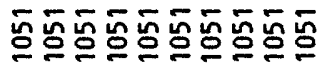

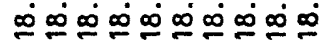

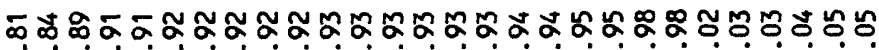

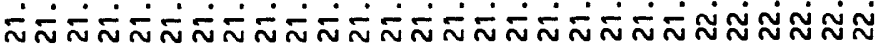

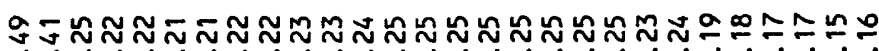

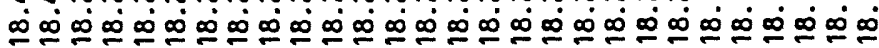

ㄴํำง

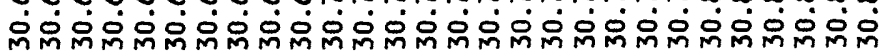

$\hat{0}$

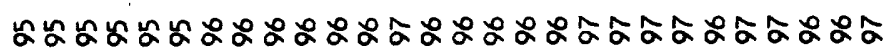

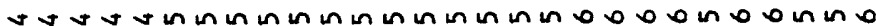

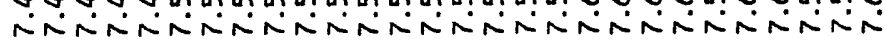

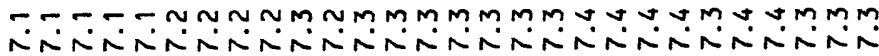

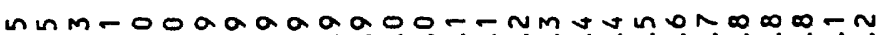
นกั่ง

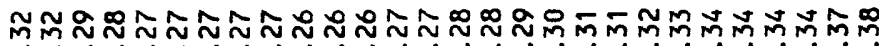

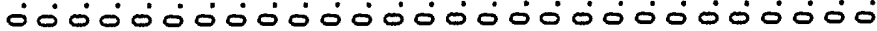

0000000000000000000000000000 $\therefore$ तm

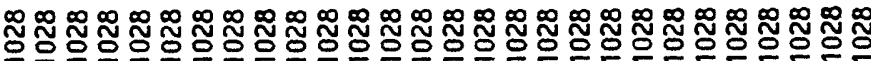

0000000000000000000000000000

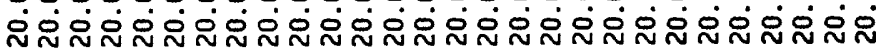


s.

늉요

a

,

:

It

in mo

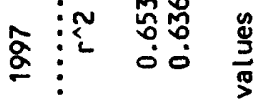

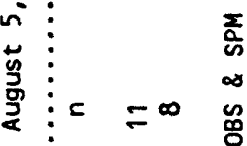

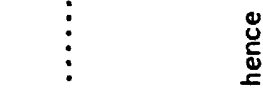

!

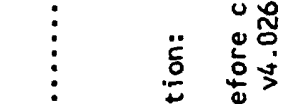

命

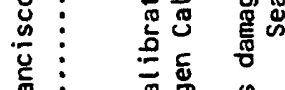

跑

员

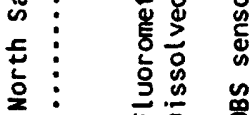

: 正哭 


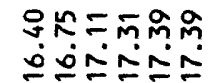

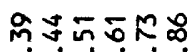

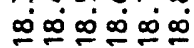

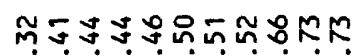

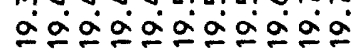

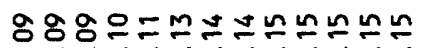
$\mathcal{3} \mathcal{y}$

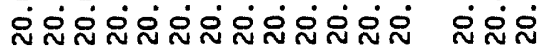

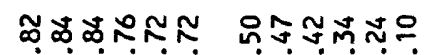

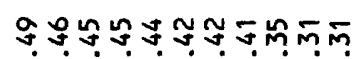

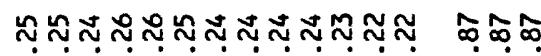
กี่ $\tilde{N} \tilde{N} \tilde{N}$
กี่ำกำ

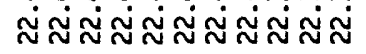

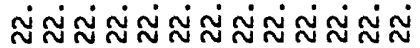
$\dot{\sim} \dot{\sim} \dot{N}$
ฆับำฏ

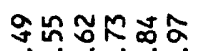
テ
究

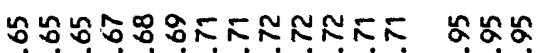

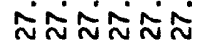
ฉ

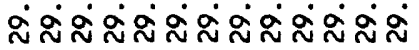
ลั่จ
$\stackrel{0}{9}$
$\stackrel{\sim}{\sim}$
$\stackrel{\sim}{\sim}$

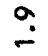

(1)

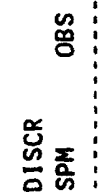

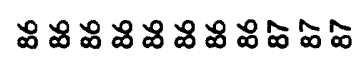

mivivivivis

ợ̂n

mmmmmmmmmsm

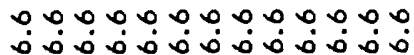

m.

in

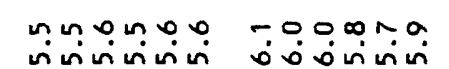

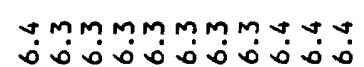

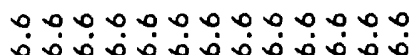

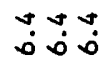

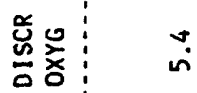

3

㝴壱

åaoํำ

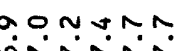

은은

ธุตำํํํํํำ

$\because-\therefore-\dot{-}$

ำ용ㅁำ

00000

बें

吾亭

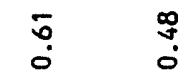

$\stackrel{\circ}{=}$

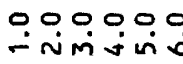

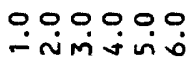

崖

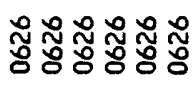

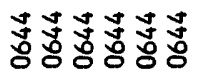

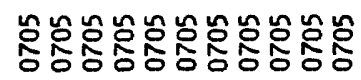

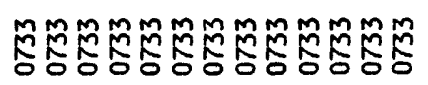

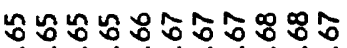

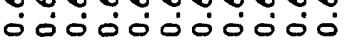

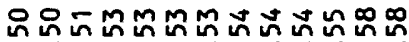

o00000000000

0.00 $\begin{array}{lll}n & 0 & 0 \\ 0 & 0 & 0\end{array}$

n?

0.0000 .0000$.

- imivivinas=

0000000000000000 -

孞

000000

0.0 .000$.

0.00000000000

0.000000000000

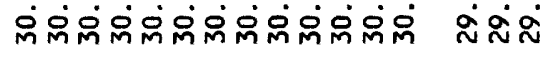

0.0

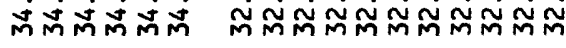




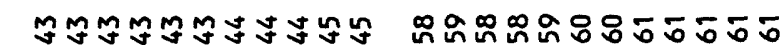

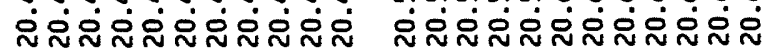

mRRTRRRㅗ.

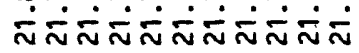

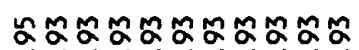

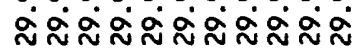

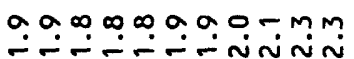

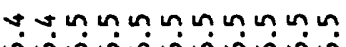
0000000000000

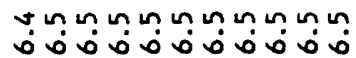

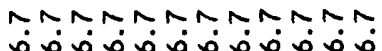

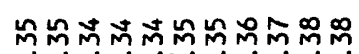
0000000000

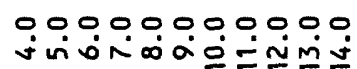

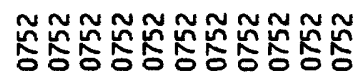

\subsection{0 .000 .000$.}

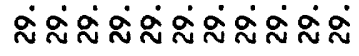

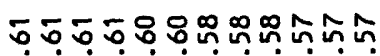

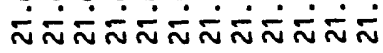
송승용ㅇㅇㅇㅇㅇㅇㅇㅇㅇ

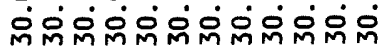
$\stackrel{\circ}{i}$

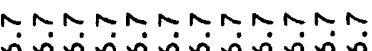
$\dddot{0}$

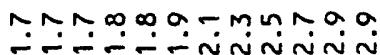

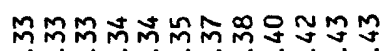
000000000000

ธั

$\stackrel{丶}{\circ}$ $\stackrel{\sim}{\sim}$

$\stackrel{i}{i}$

000000000000 -

$\cong \cong \infty \infty ⿻ \infty$

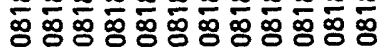

0.00000000000

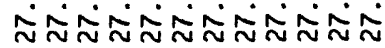

0,0000000 -imivionio

ํํํํํํํํํํำ

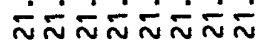

NTKNTKRS

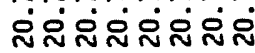

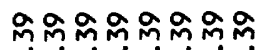

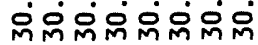

$\stackrel{?}{-}$

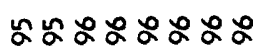

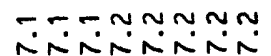

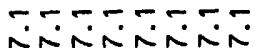

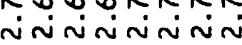

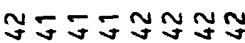
00000000

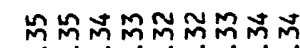
00000000

$\stackrel{?}{\circ}$

$\stackrel{0}{i}$

000000000 - imiviviso

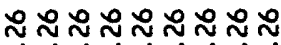

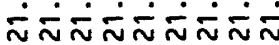

๓ะヘ ニ ํํํำ

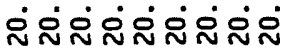

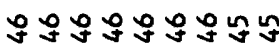

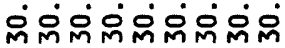
$\stackrel{m}{-}$

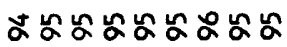

- - nNNNNNN mañañ $\bar{i}$

$00 \infty 0000 N \infty$

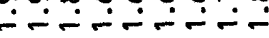<smiles>[13CH3]</smiles>

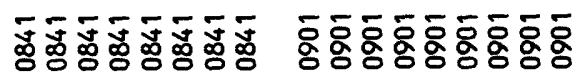

00000000

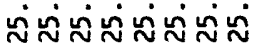

000000000

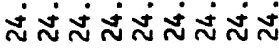




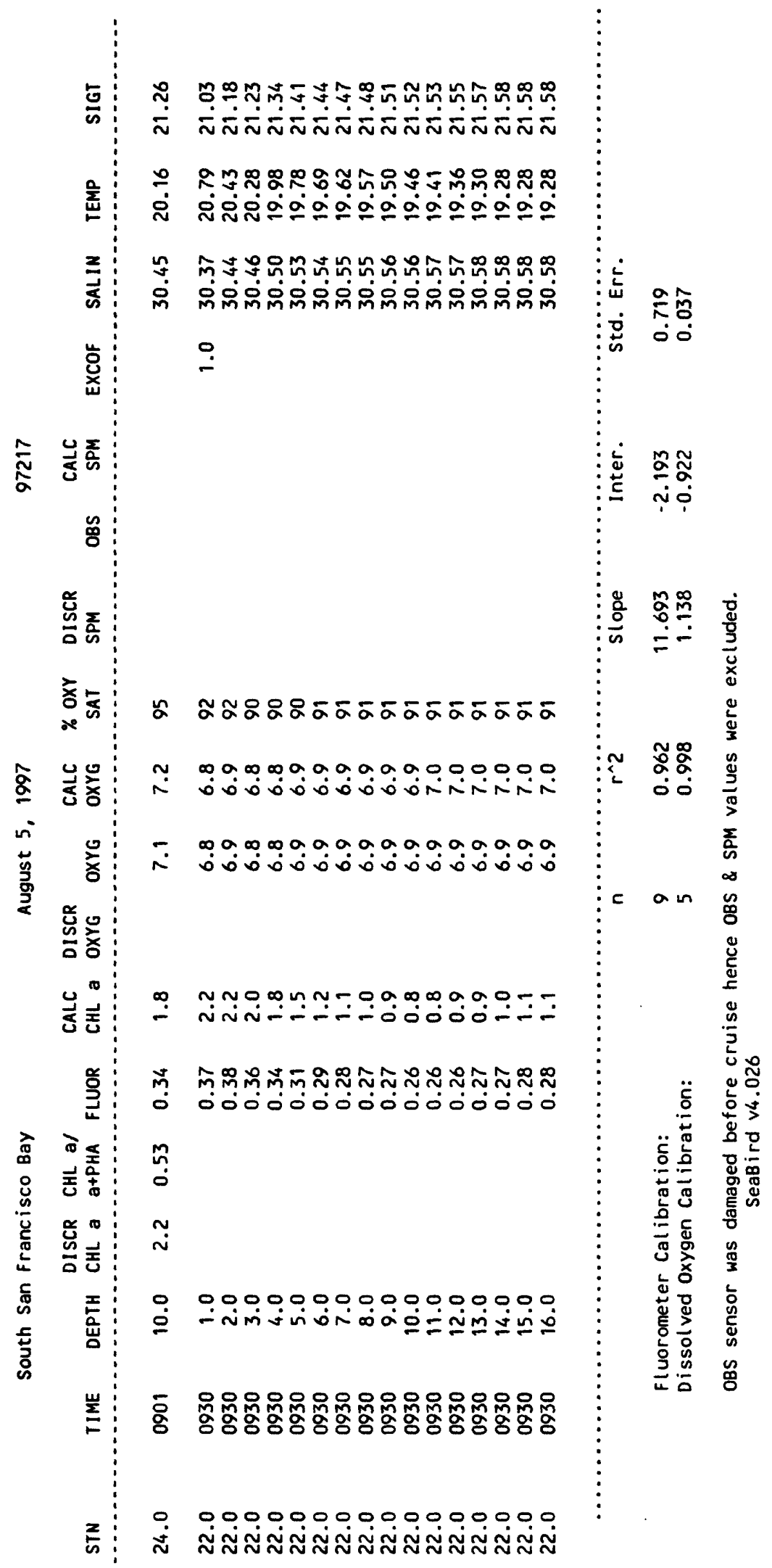


8888888888.8888888888

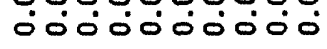

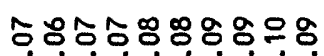

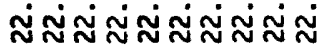

으으으으으으으으으으

ऽ00000000

$\stackrel{+}{\square}$

స్్

爮

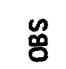

苟

$\simeq \simeq \Xi ニ=\mp \simeq \simeq \simeq \simeq$

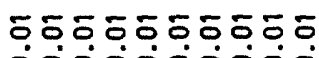

व००000000

$\stackrel{\circ}{\circ}$

爻產



逐文

迹

彥

음

起

9090909090

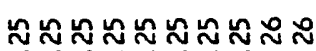
ธ000000000

$\tilde{a}$

$\because$

0.000000000

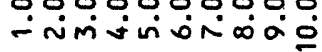

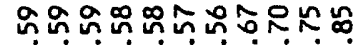

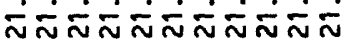

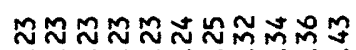
0000000000

$\dddot{n}$

ละกส

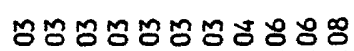

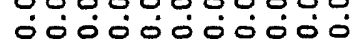

$\stackrel{\sim}{\sim}$

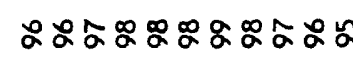

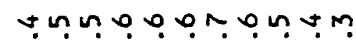
$\infty \infty_{\infty}^{\infty} \infty \infty_{\infty}^{\infty} \infty \infty_{\infty}^{\infty} \infty \infty_{\infty}^{\infty}$

บุ?

$\infty \infty \infty \infty \infty \infty \infty \infty \infty \infty$

$\stackrel{+}{\infty}$

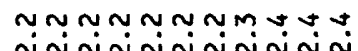

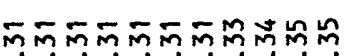
0000000000

$\overline{0}$

$\stackrel{\circ}{\circ}$

$\stackrel{8}{\circ}$

$\stackrel{\leftrightarrow}{0} 0$

$\stackrel{\circ}{\sim}$

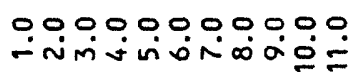

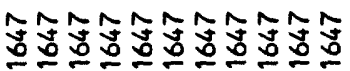

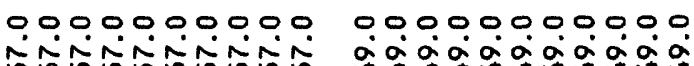

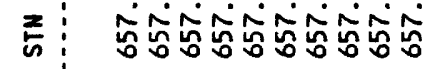

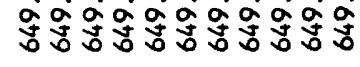

88888888888

000000000

88888888

00000000

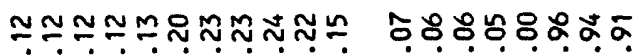

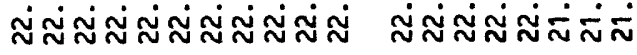

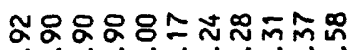

ஸ்0ல்ப்-ப்-口

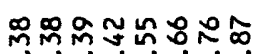

$\stackrel{n}{n}$

$\stackrel{\circ}{\sim}$

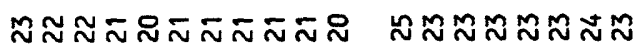

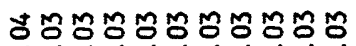

000000000

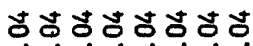

00000000

$\stackrel{\circ}{\sim}$

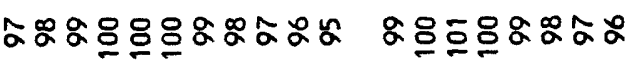

t

$00,0, \pi n$ $\infty \infty^{0} \infty \dot{\infty} \infty \dot{\infty} \infty \dot{\infty} \infty \dot{\infty} \infty \dot{\infty}$

$\dot{\infty} \dot{\infty} \dot{\infty} \infty \dot{\infty} \infty$

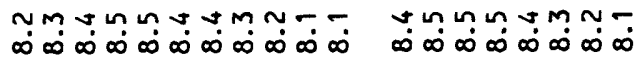

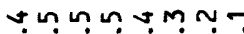

$$
\stackrel{+}{\infty}
$$

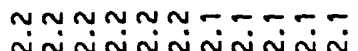

00000000 minananina nininini--

ตับ๊ี ०0ं0000000

กึ心 வ0ं00000

กัo

กิ

00000000000

iniminínió

00000000

- imisisio

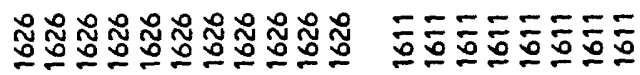

0000000000000000000 iñinininininin mimimimimi 
88888888 ธำำำกำ

$\circ 0^{\circ}$

00000000000

๓๐口 โMร์

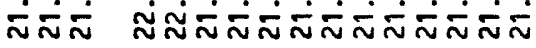

ธํำำ

inin

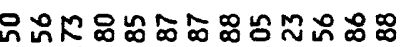

iñinininimmimm

$\stackrel{\circ}{\text { i }}$

$\because \infty$

กุกั

ฟกำก

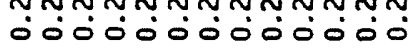

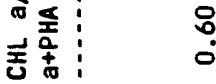

总 동

좀

崖

옹음

-

Б๐๐

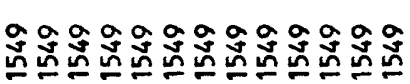

$\bar{i}$

జ

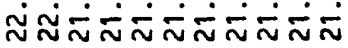

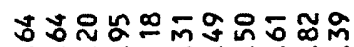

minis ivinivinivisio

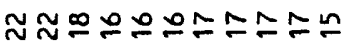

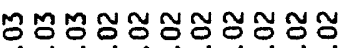

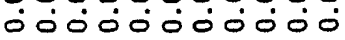

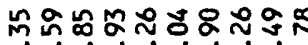

iñinivisivinis

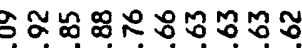

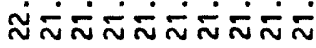

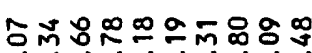

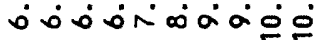

m

$\stackrel{\square}{-}$

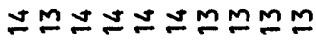

$\simeq \simeq$

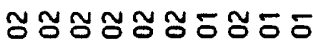

0ं0000000

$\overline{0}$

$\stackrel{\infty}{0}$

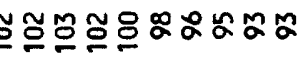

쏭응

舟

กุ

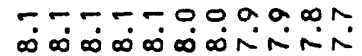

$m m$

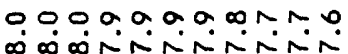

t t n v n o a n o o $\dot{\infty} \dot{\infty}$

$$
\stackrel{0}{\infty}
$$

n $-\infty$ onnmunn?

rom om and a d a d

in

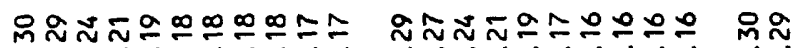
0000000000 00000000 00

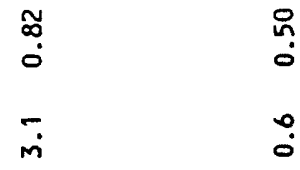

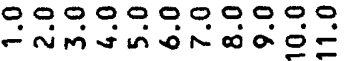

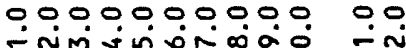

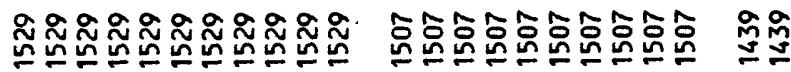

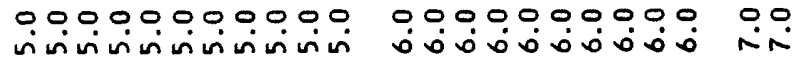

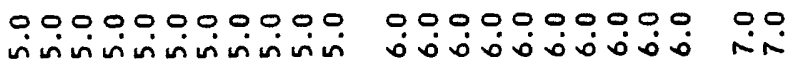




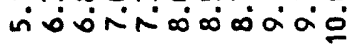

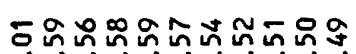

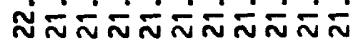

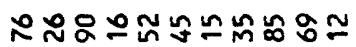

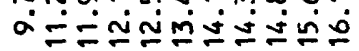

드응ㅇㅇㅇㅇ웅

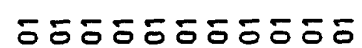
○ं000000000 (1)

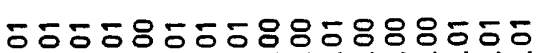
00000000000000

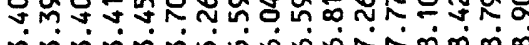

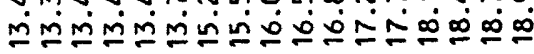
$\stackrel{9}{-}$

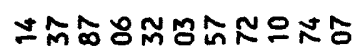

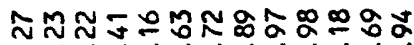

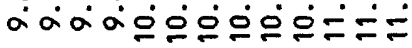

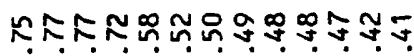

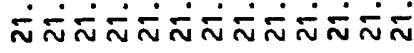

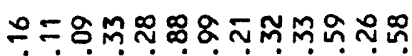

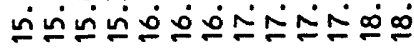

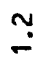

ニ응ㅇㅇำำㄴำะ

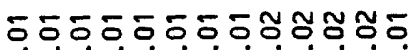
क0ं00ं00000

$\stackrel{\simeq}{\simeq}$

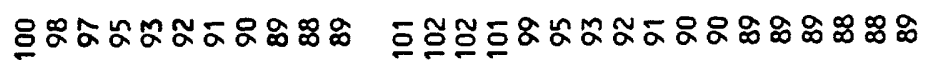

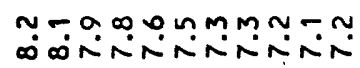

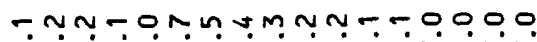

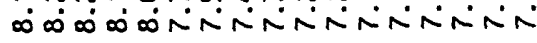

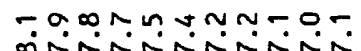

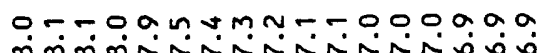
DMnNMNANA

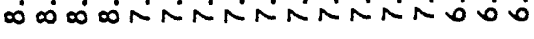

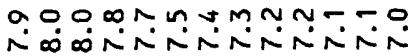
$\stackrel{a}{n}$

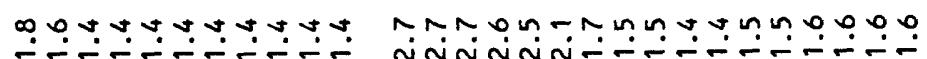
ำกำํํํำะ ல0ं0ல000000

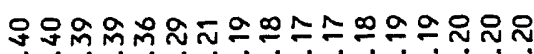
00000000000000

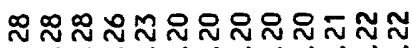
०00000000000

$\stackrel{\circ}{\circ}$

$\stackrel{9}{-}$

0.0000000000 mंงทำ

00000000000000000 -

0000000000000

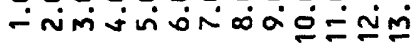

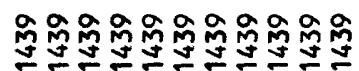

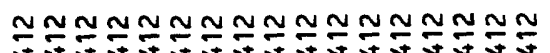

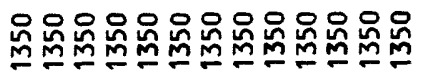

00000000000 hitivitivitio

00000000000000000

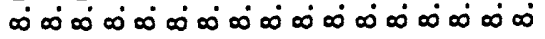

0000000000000

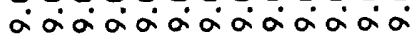




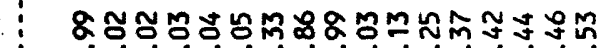

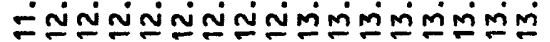

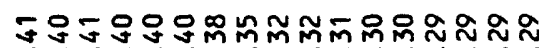

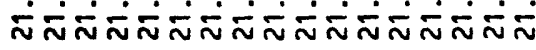

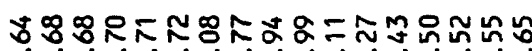

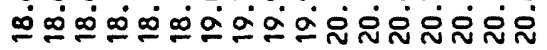

蒙

స్ aे

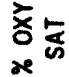

善彥

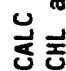

NNNRNRN

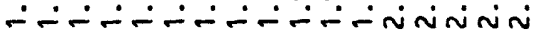

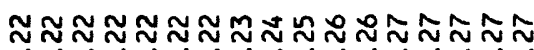
0ं0000000000000

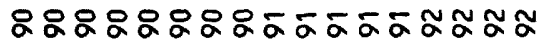

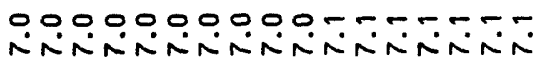

ธธธธธธธธธธธธธธธธธธ

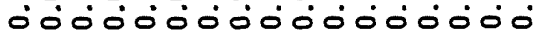

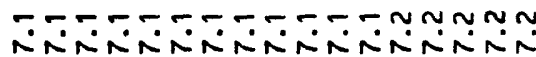

$\stackrel{\sim}{\mathfrak{0}}$

$\dddot{m}$

00000000000000000

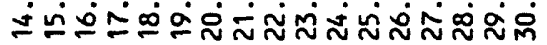

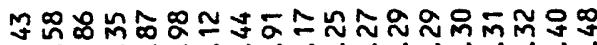

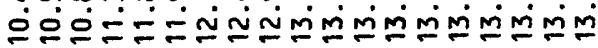

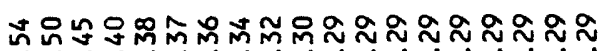

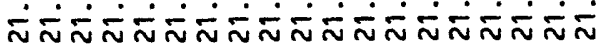

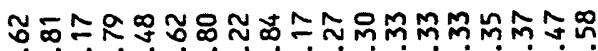

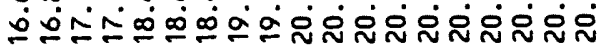

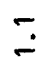

으으으으응 $00 \infty \sim \wedge \sim \sim \infty N \infty \infty N$

ธธธธธธ8858888888888

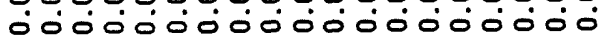

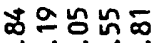

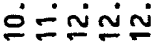

ㅇำำำ ล்亠凶்

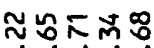

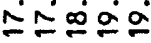

$\hat{0}$

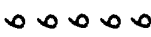

88888 0000

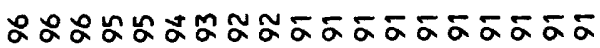

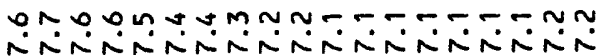

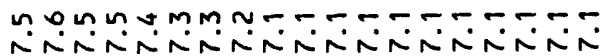

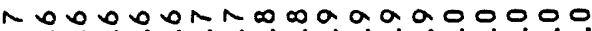

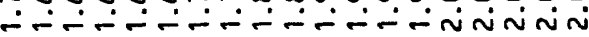

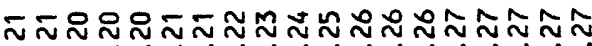

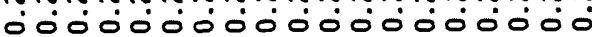

0000000000000000000

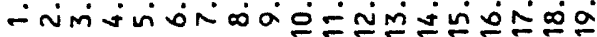

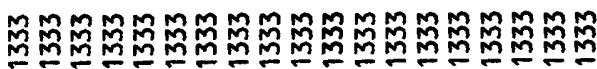

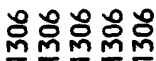

0.0 .00 - imivi

0000000000000000000

00000000000000000

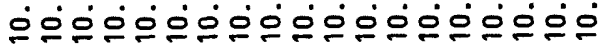

0.0 .00

$\check{\check{E} \check{E}=}$ 


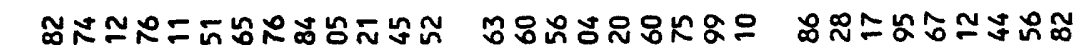

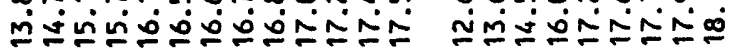

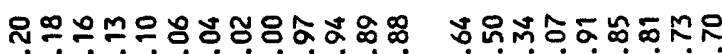

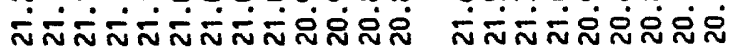

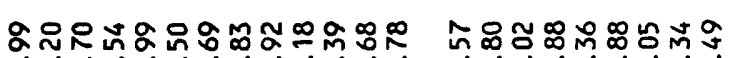

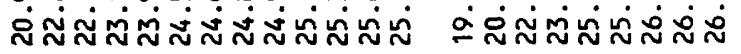

홇

స్ స్

这

ON00000000000000000000

8888888888888888888888

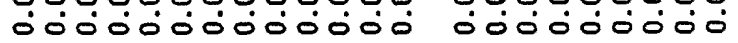

总

๙

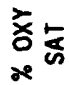

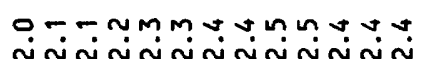

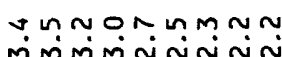

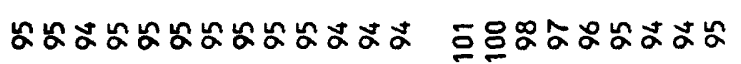

nommmmmmmmañ

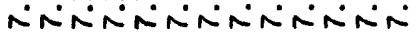

a

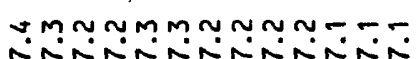

miñ

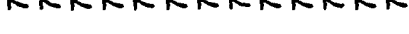

ShNnNana

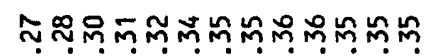
ó0000000000

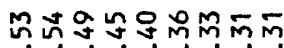

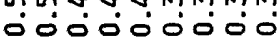

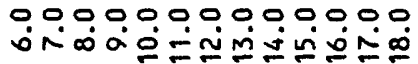

0,00000000 - imivivioiso

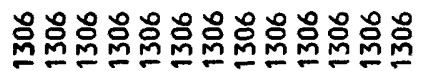

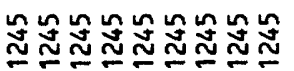

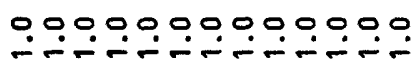

000000000

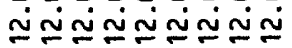

$\infty \infty n 0-a$ on

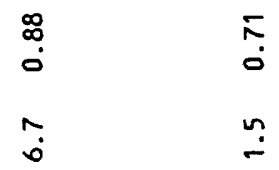

000000000 - Nimínín

a a a a a a a a

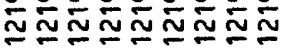

000000000

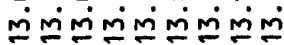

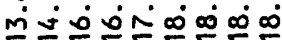

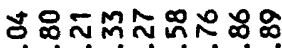

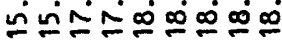

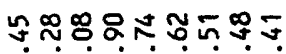
ஸ்亠்

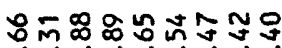

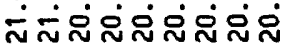

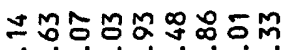

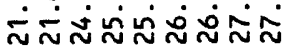

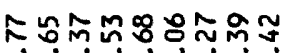

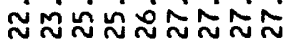

ณ?

$\ddot{0}$

000000000

000000000

888888888 லं0்0ं0்

888888888 ódiojó0

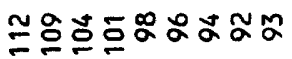

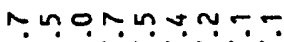

$\infty \infty \infty N N N$ mmañ $\stackrel{\infty}{\infty}$ ijminini

ONN $\infty$ man

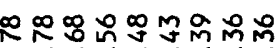
0ं00்000

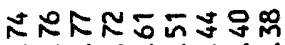
00000000

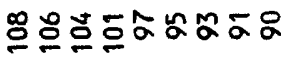

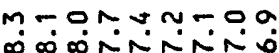

-Oa ovnoan

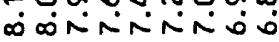

000000000 - Nimivioño

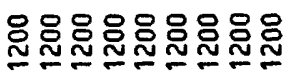

000000000

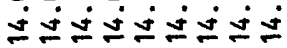


ที

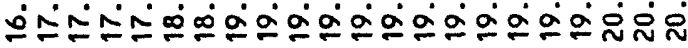

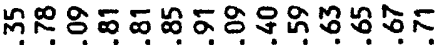

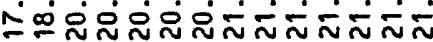

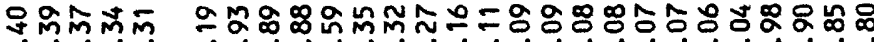
这怘怘怘

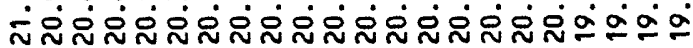

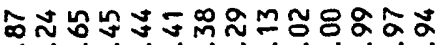

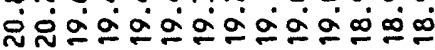

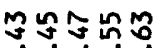

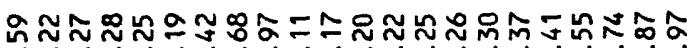

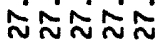

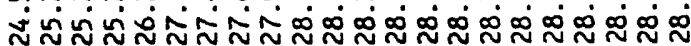

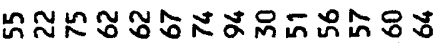
ผั่

0000000000000000000000

응옹응용응

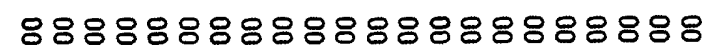
$\therefore \dot{0} 0$.

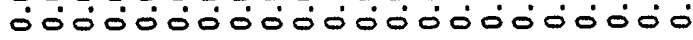
$\stackrel{\sim}{m}$

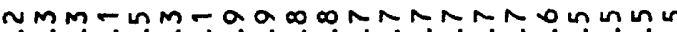

$\log \ln \ln \sin$ iñini

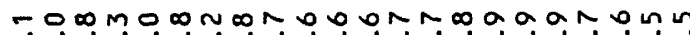

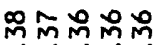
ऽั0்

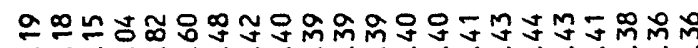

$\stackrel{8}{\circ}$

$\circ$

in

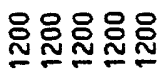

\section{$\infty$}

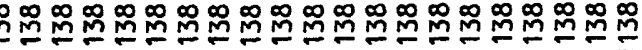

0.0 .000

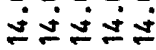

$\tilde{0}$

00000000000000

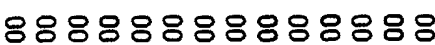

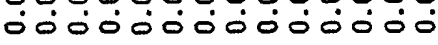

unammnmm- 0 ond ¿

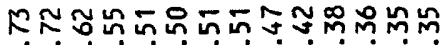
0ं0்000000000 $\tilde{0}$

$\stackrel{0}{0}$
00000000000000 -

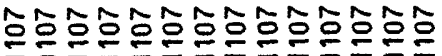




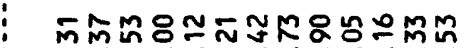

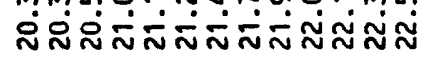

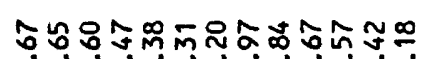

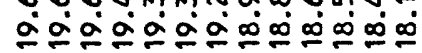

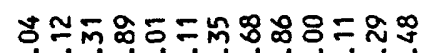

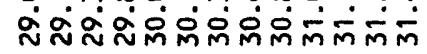
ó

0000000000000

8888888888888 000000000000

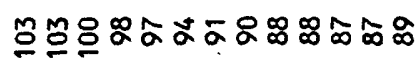

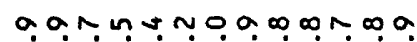

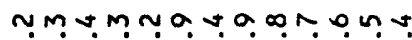
iqugimiminiñiñ

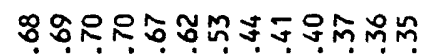
000000000000

0.00000000000

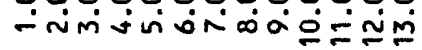

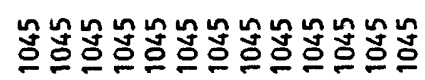
0000000000000 논

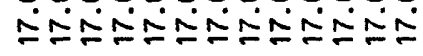

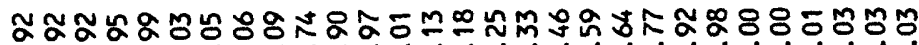

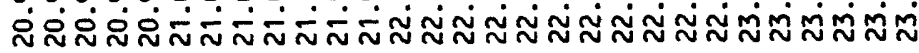

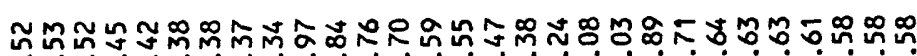

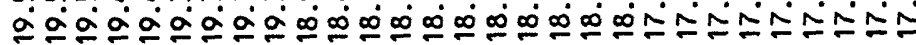

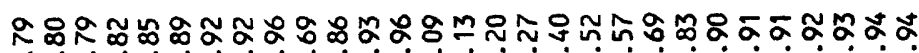

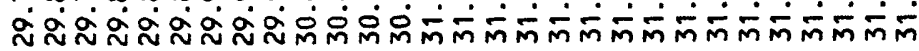
$\hat{\circ}$

00000000000000000000000000000

88888888888888888888888888888

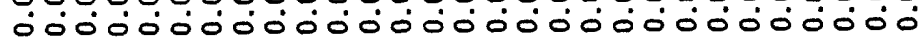

$\tilde{n}$

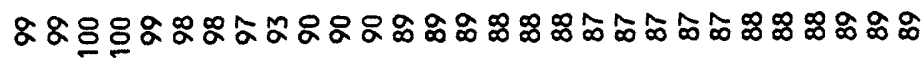

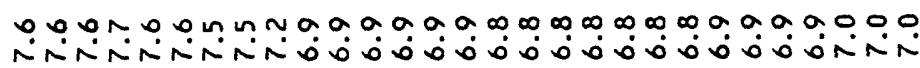

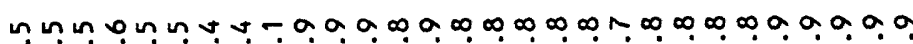
rinariniso

$\hat{n}$

Y แ juj

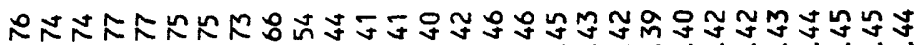
00000000000000000000000000
$\stackrel{m}{\mathfrak{m}}$
m.:

00000000000000000000000000000

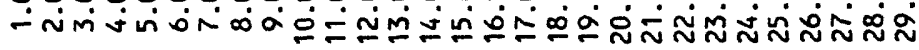

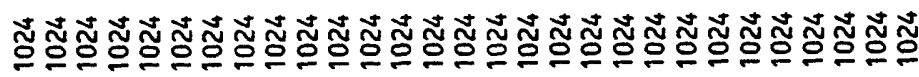

00000000000000000000000000000

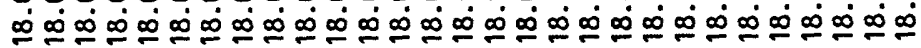




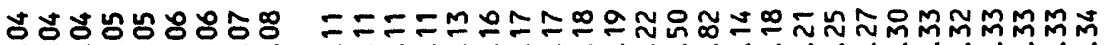

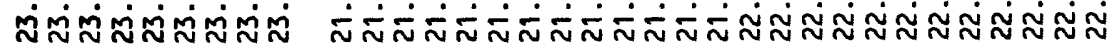

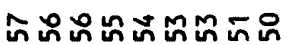

๑ి

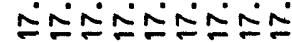

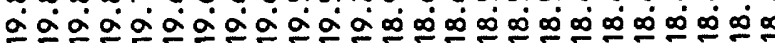

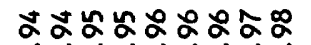

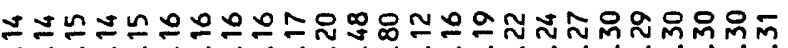

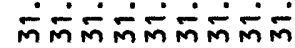

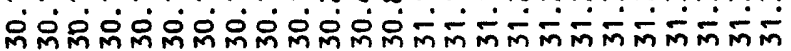

$\stackrel{\circ}{\circ}$

ธָฐฒ心

$\therefore \sim$

000000000

保思

888888888 8888888888888888888888888

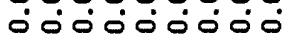

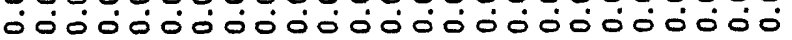

$\stackrel{8}{8}$

总

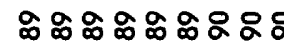

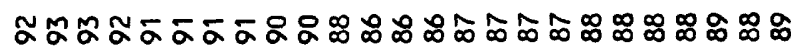

0.000 .0007 .0

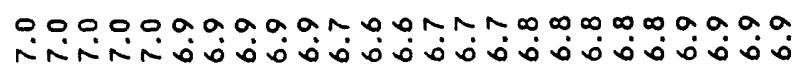

000000000

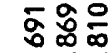
ல்

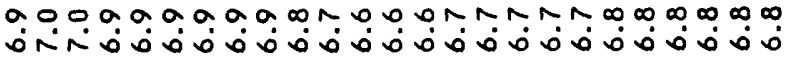

ioginitin

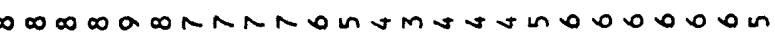

$a r 0 \infty \infty a r-0$

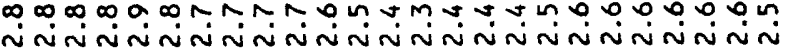

옹 존

nimininimimim

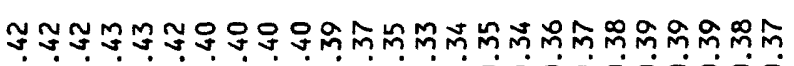

ที่ง

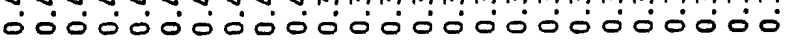

0000000000000000000000000

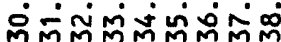

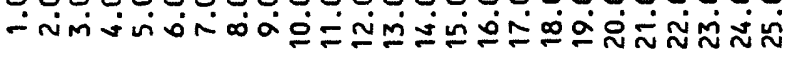




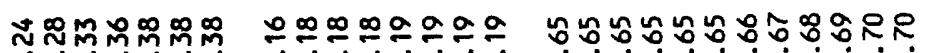

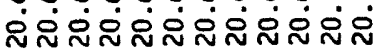

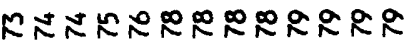

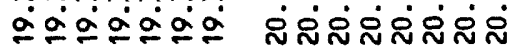

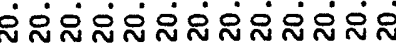
으유으으으 응ㅇ읃ㅇㅇㅇㅇㅇㅇ

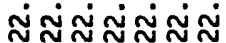

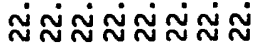

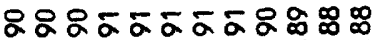

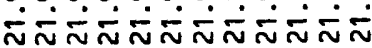

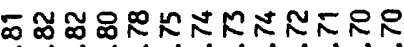
ஸ்ஸ்

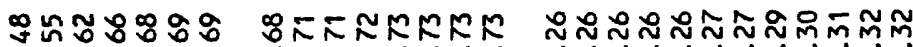

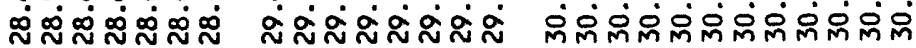

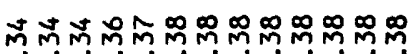
อิं

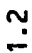

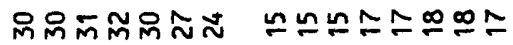

$\infty \infty \infty \sim \infty a a+=\simeq m \simeq$

0000000000000

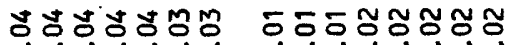

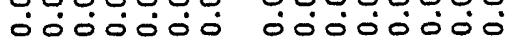

$8888858 \overline{5} \overline{0} \overline{0}$ वó00000000

8888888888888

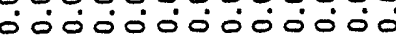

$$
\text { m. }
$$

$\stackrel{+}{\infty}$

ษ

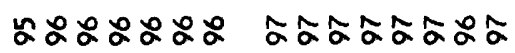

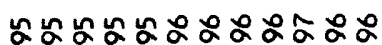

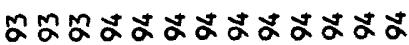

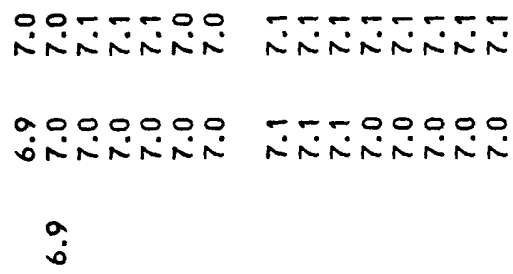

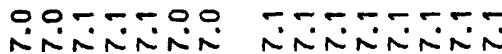

0000000 -7.00000

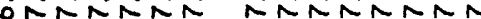

$$
\text { a. }
$$

$0.0 .000 .0007=$

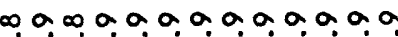

a.0000000000

$\stackrel{\circ}{i}$

O̊m

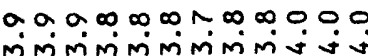

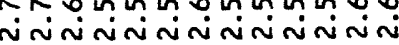

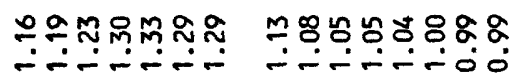

$\stackrel{n}{0}$

$\stackrel{9}{?}$

$\stackrel{\infty}{\sim}$

总

$$
\stackrel{\infty}{\sim}
$$

ă

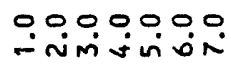

00000000 - imivinisio

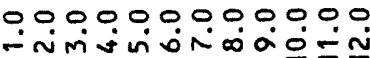

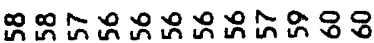
00000000000

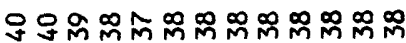
0000000000000

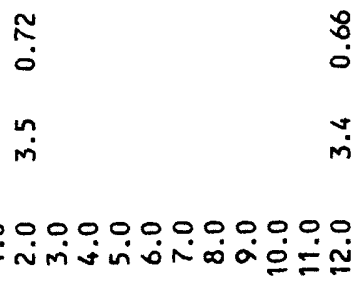

$\tilde{0}$

m

崖

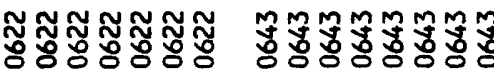

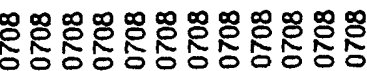

0000000000000

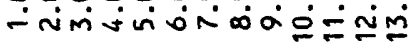

\subsection{0}

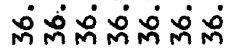

0.000000.

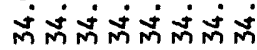

000000000000

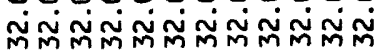

0000000000000

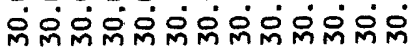




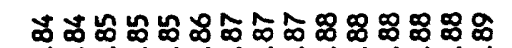

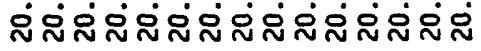

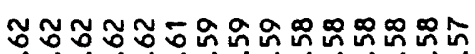

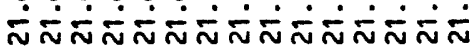

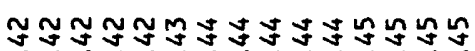

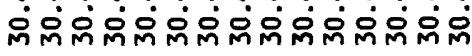

$\stackrel{\square}{\square}$

స్్

㝴

000000000000000

888888888888888

- 00ं000000000

芯

ชัญ



$a^{\circ}$

ليّة

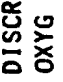

通突

NND000000000000

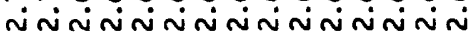

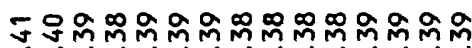

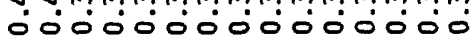

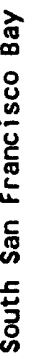

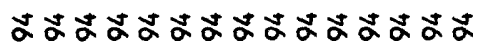

9.9090909090909

$\infty \infty \infty \infty \infty \infty \infty \infty \infty \infty \infty \infty \infty \infty$

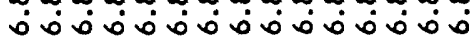

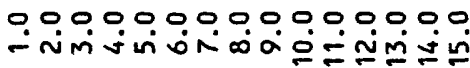

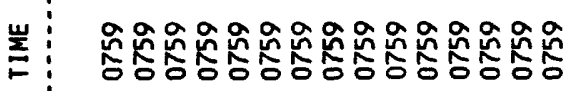

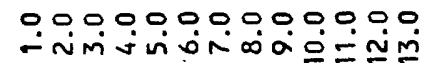

8888888888888

-0000000000

$\stackrel{j}{4}$

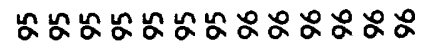

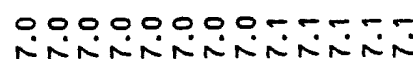

aoaoaaa000000

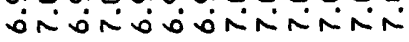

$\bar{i}$

aOa00000- NNMM

imiñiñiminimimimim

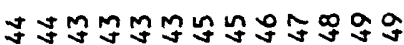
क००000000000

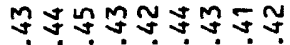
00000000

พิกฺฺฺ

$\tilde{a}$

$\stackrel{5}{0}$

m.

$\tilde{m}$

888888888888

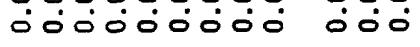

$\stackrel{\circ}{\sim}$

$2000 \infty 0 a \infty \infty$

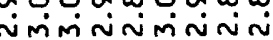

inis

$\stackrel{\infty}{\infty}$

0.0000 .000

- imivivinió

울ํํ

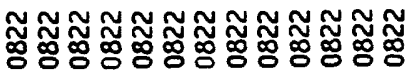

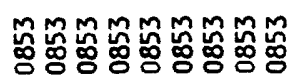

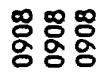




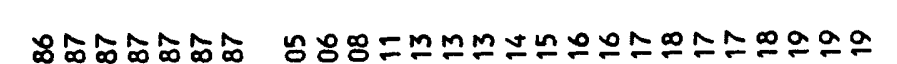

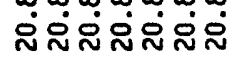

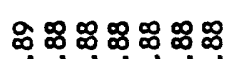

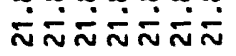

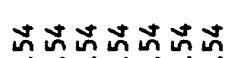

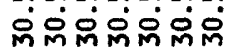
(1)

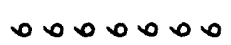

8888888 0000000

8888888888888888888

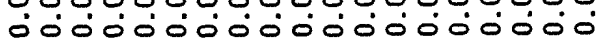

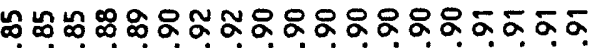

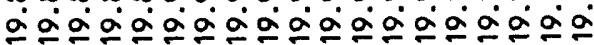

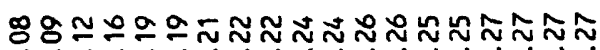

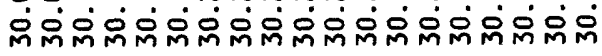
亏.

0000000000000000000

呚

迄

ํํㅇ 总

总至

goginimm

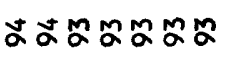

$\infty \infty \infty \infty \infty \infty \infty$ $\dot{0} \dot{0} \dot{0} \dot{0} \dot{0}$

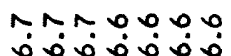

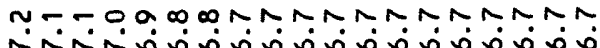

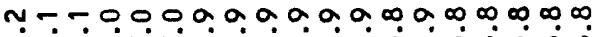

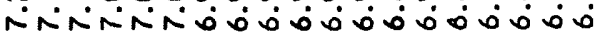

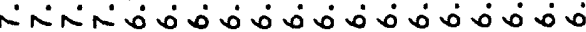

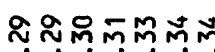
000000

$\stackrel{0}{0}$

$\bar{m}$

웜롱

돔

0000000

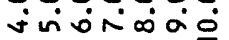

0000000000000000000

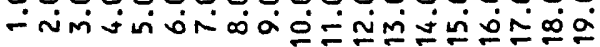

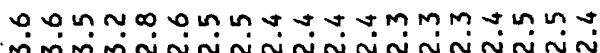

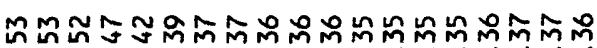
0000000000000000000

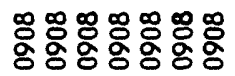

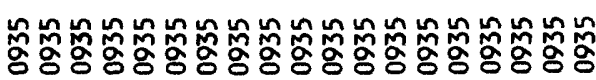

0.0 .0 .0 .00

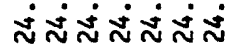

$0.000 .000 .00,000,0.000 .000$

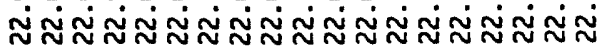

ปัర์

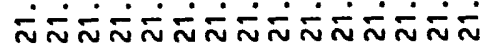

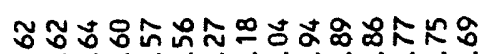

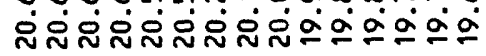
ผิ

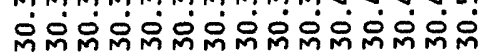

$\hat{0}$

000000000000000

888888888888888 ல0000000000000

$$
\stackrel{0}{0}
$$

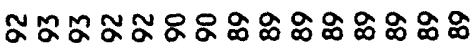

a $000 \infty \infty \uparrow \uparrow \infty \infty \infty \infty \uparrow N$ ดी

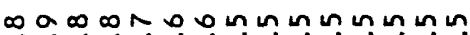

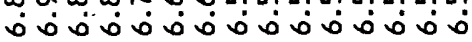

$\stackrel{a}{0}$

mmmmmnam mimmmininininiñon

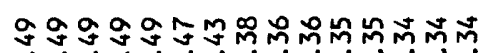
0000000000000

$\bar{\infty}$

ळ

$\stackrel{m}{m}$

000000000000000

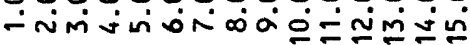

000000000000000

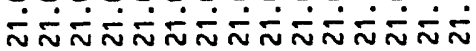




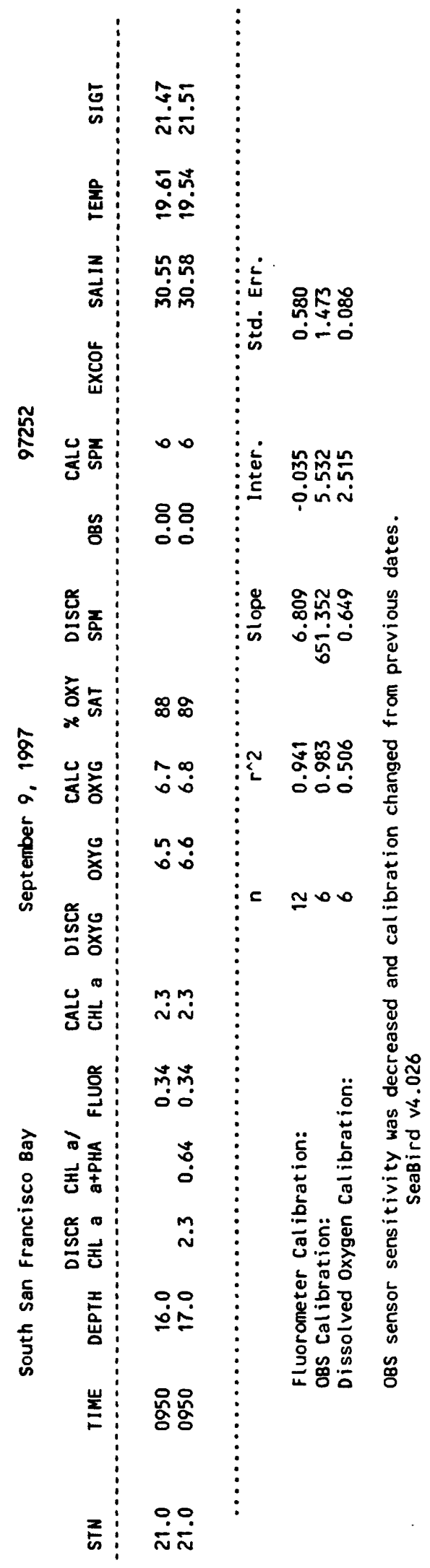


1888888888 ลตัดำกำกำก

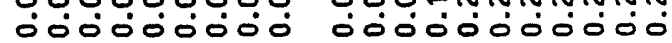

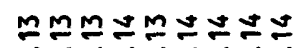

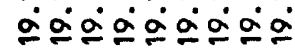

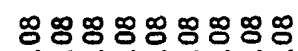
000000000

!

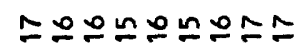

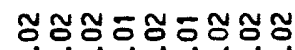
ல0ं0ं0ं00ं0

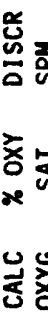
N

崖

000000000 z:

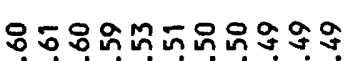

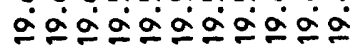

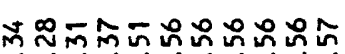

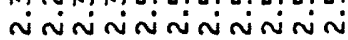

$\cong$

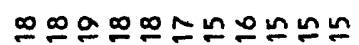

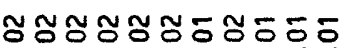
वं00ं00000

$\stackrel{m}{\infty}$

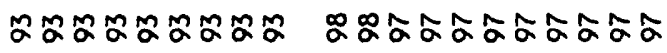

$00 \% 00000 \%$

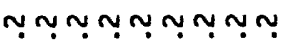
مَّ

$\overbrace{\infty}^{n}$

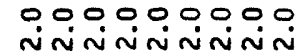

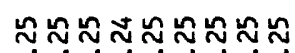
000000000

๕.

0

$\bar{i}$

000000000 - imivivinio

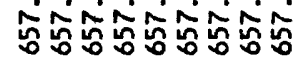

$\infty \infty \infty\{?\{?\{\} ?$

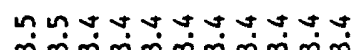

$$
\tilde{\infty}
$$

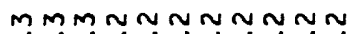
ñññinaññ

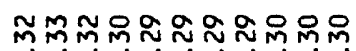
ó00ं00ं0000

$\tilde{\tilde{O}}$

$\stackrel{9}{-}$

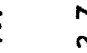

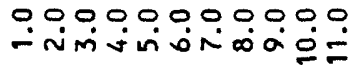

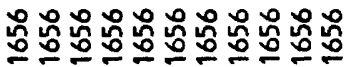

00000000000

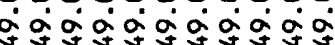

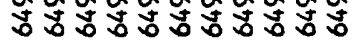
$\infty \infty_{\infty}^{\infty} \dot{\infty} \dot{\infty} \dot{\infty} \dot{\infty} \dot{\infty} \dot{\infty} \infty \dot{\infty}$

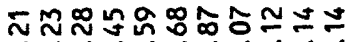

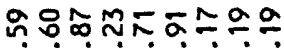

-ெ-口-

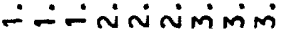

눈ㅈNำ

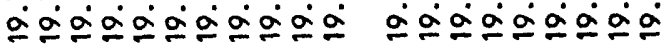

๓

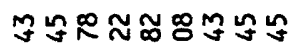
mimiojojojinini

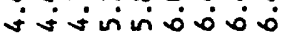
$\dddot{乛}$ $\stackrel{m}{9}$

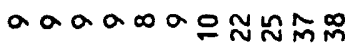

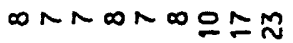

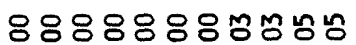
0ं000000000

$8888888 \%$

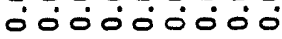

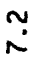

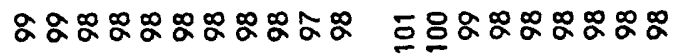

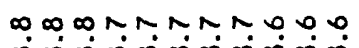
$0, \infty \sim N \infty 000$

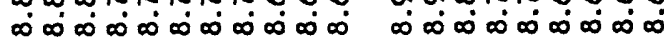

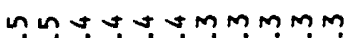
$\infty \dot{\infty} \infty \dot{\infty} \infty \dot{\infty} \infty \dot{\infty} \infty \dot{\infty}$

$0 \infty m m \sim N n$ $\infty \infty \infty \infty \infty \infty \infty \infty \infty \dot{\infty}$

$$
\stackrel{0}{\infty}
$$

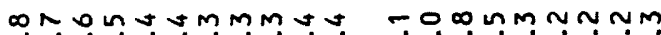

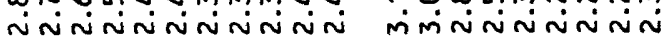

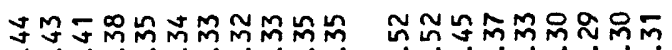

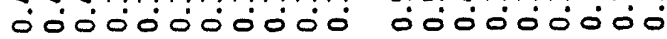

$\stackrel{\infty}{\infty}$

$\dddot{v}$

00000000000000000000

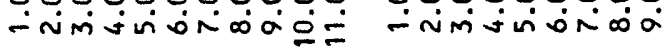

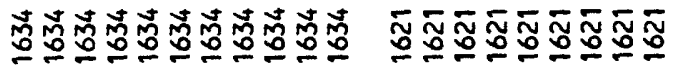

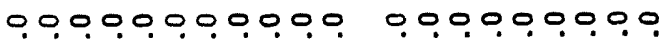

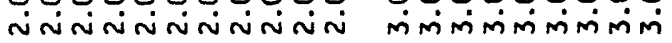




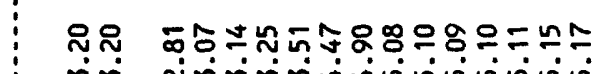

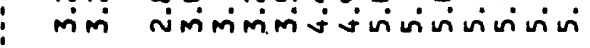

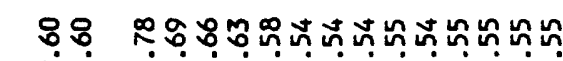

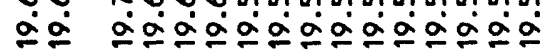

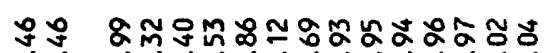

०० viि

$\doteqdot$

蛋 造

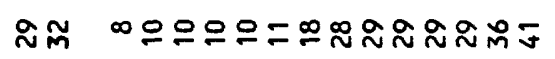

ปั 8 ป

í 000000000000

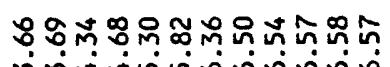
minưvivio

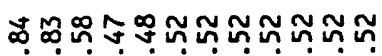

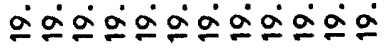

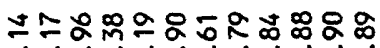

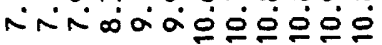

$\stackrel{m}{?}$

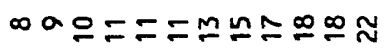

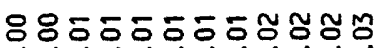
की0ं0000000

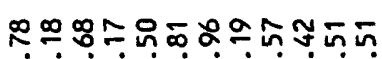

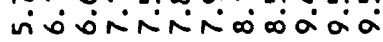

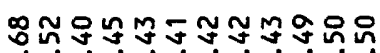

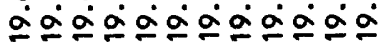

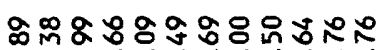

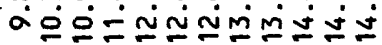
$\cong$

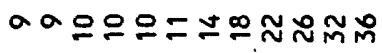

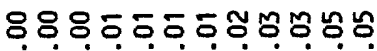
هั0ं0ं00ं00

\section{$\stackrel{0}{a}$}

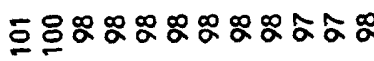

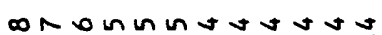

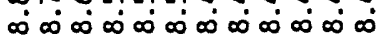

nmn- - $000 a a a$

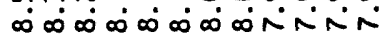

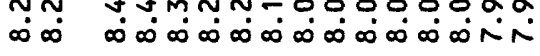

0

$\stackrel{2}{2}$ N

음

岁곤

min

m.m.

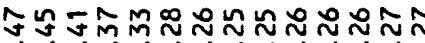
0000000000000

anonmn-00-...

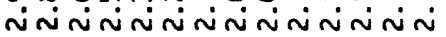

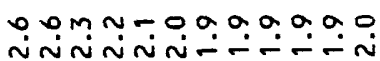

ن.

Goำ 00000000000

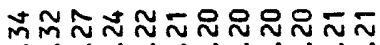
00000000000

ก

@

$\stackrel{\sim}{\sim}$

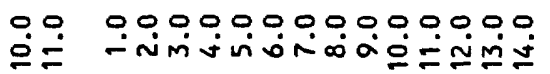

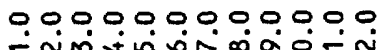

$\stackrel{\circ}{\circ}$

กิ

$\hat{i}$

I

$\infty \infty \infty \infty \infty \infty \infty \infty \infty$

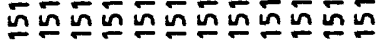

000000000000 vinivivivinivivivivivin

000000000000

웅우웅우웅웅우웅우 -nmunundogen 0000000000000 


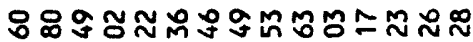

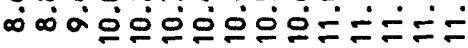

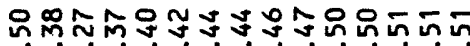

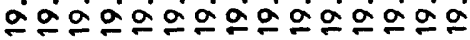

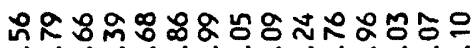

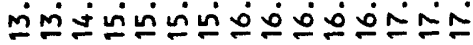
$\dddot{m}$

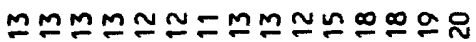

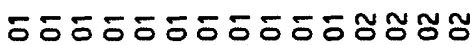

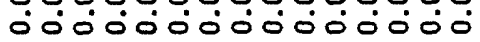

䢟

ชัญ์

๙

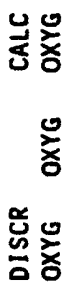

爮志

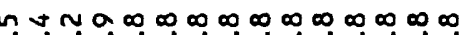
ن

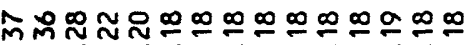

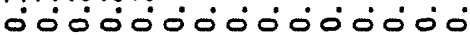

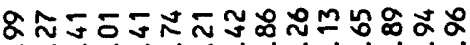

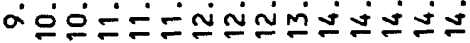

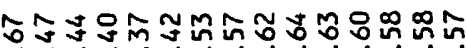

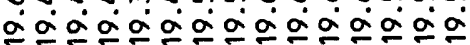

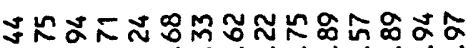

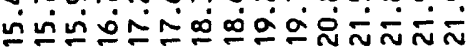

$\stackrel{m}{9}$

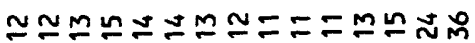

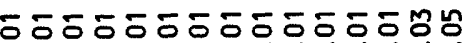

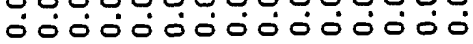

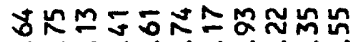

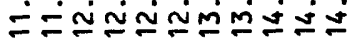

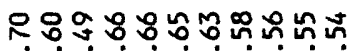

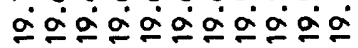

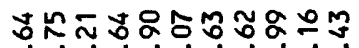

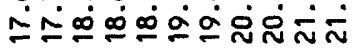

5

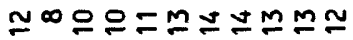

Б8885ธธธธธธ

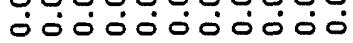

$\stackrel{9}{0}$

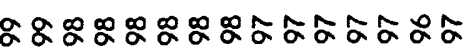

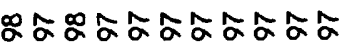
000000000000000

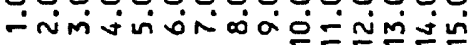
$\infty \infty \dot{\infty} \infty \infty_{\infty}^{\infty} \infty \dot{\infty} \infty \dot{\infty} \infty \dot{\infty} \infty \dot{\infty} \infty \dot{\infty}$
ก

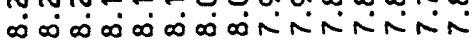

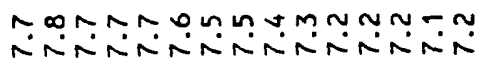

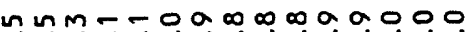
ininiñ-

mొmฺ๊ -000000000000

000000000000000

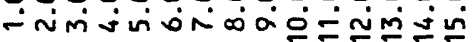

00000000000 - imivioñó丶

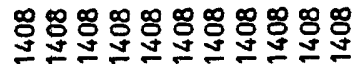

000000000000000

$0.0 .0 .0 .0,0 \infty \infty$

m n m $\stackrel{\circ}{\infty}$

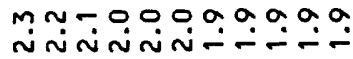

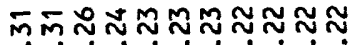
00000000000

ई.

$\bar{i}$

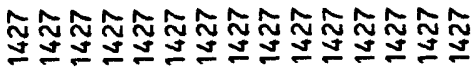

00000000000 $\infty \infty \infty \infty \dot{\infty} \dot{\infty} \sim N N$ 


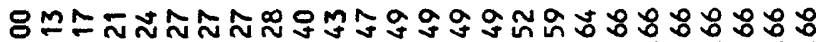

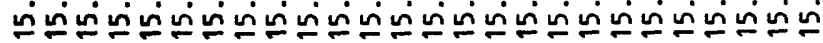

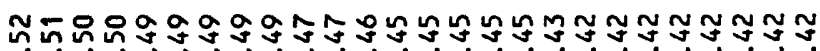

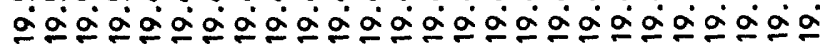

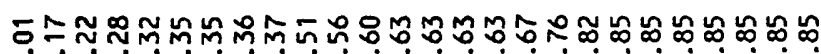

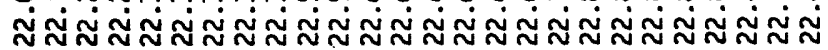

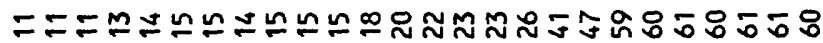

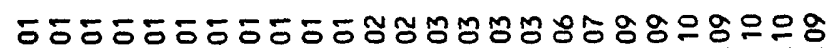

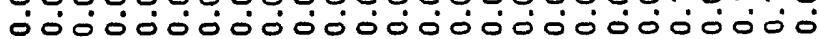

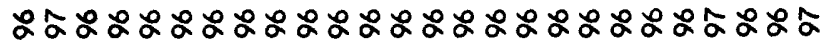

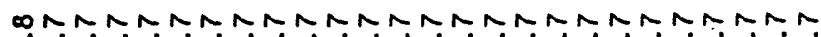

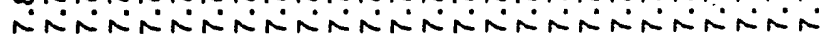

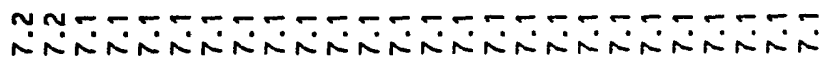

롱론

$00000000000-7-7-7-\pi n n N N n N$

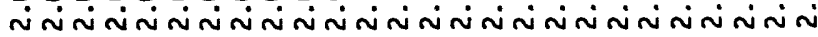

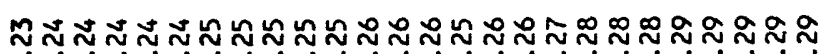
000000000000000000000000

ì.

$\stackrel{\infty}{-}$

00000000000000000000000000

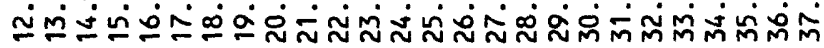

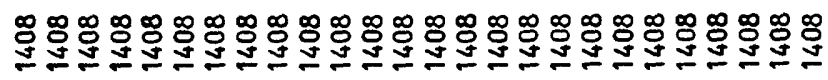

00000000000000000000000000

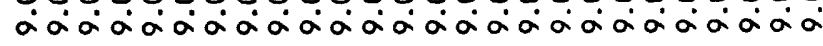

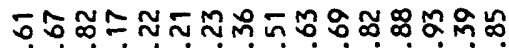

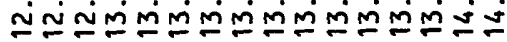

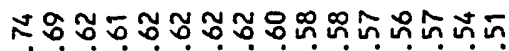

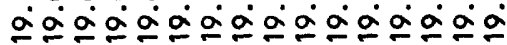

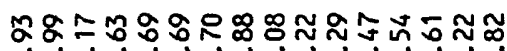

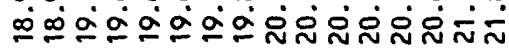

$\Xi$

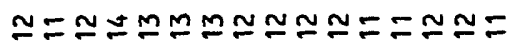

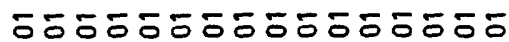
0000000000000

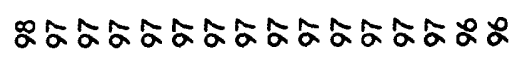

aadaaaa $0 \infty \infty \infty \infty \infty \infty \infty$

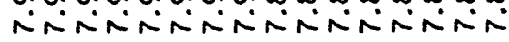

SOSMMMMMMMMMMNNN

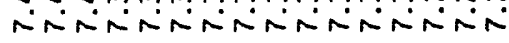

- $000 \infty \infty \infty \infty \infty a 00000$

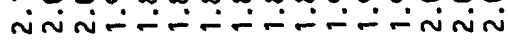

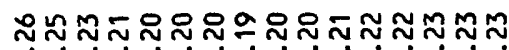

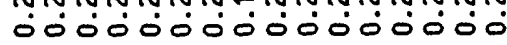

0000000000000000

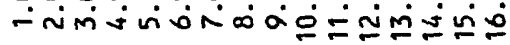

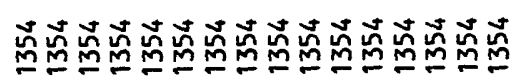

0000000000000000

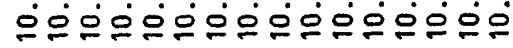




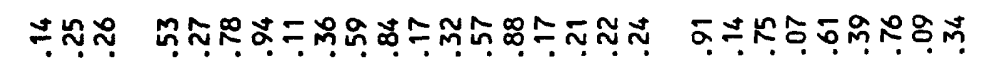

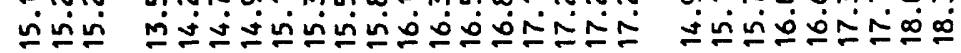

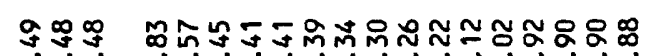

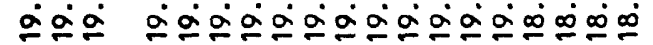

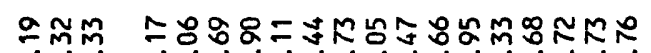

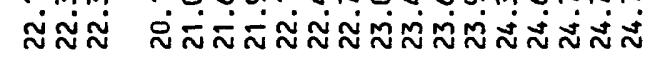

$\stackrel{-}{\circ}$

$= \pm \stackrel{\infty}{\infty}$

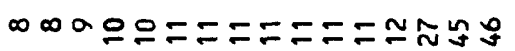

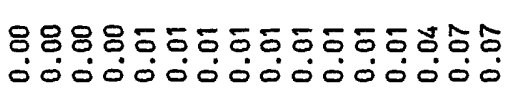

ஜ̂:

善

ชิธ์

ڤ.

ก

:

음

똟롱

운우

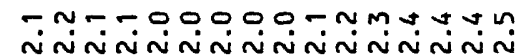
ioj

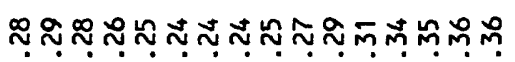

0000000000000000

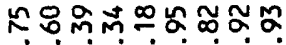

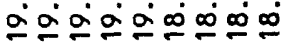

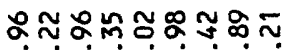

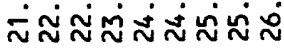

$\stackrel{\circ}{\circ}$

으오으으든

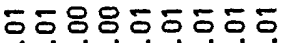
0ं0ं0ं0்0ं

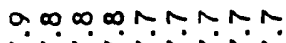

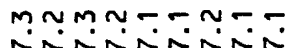

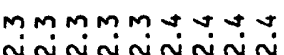

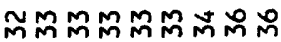
¿0000000 $\leadsto$ N

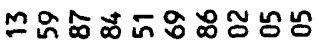

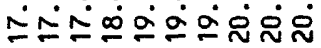

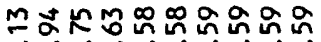
$\underline{\infty} \oplus \infty \oplus \infty \omega \infty \omega$

ดัปูป์

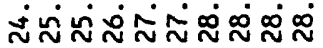

$\stackrel{0}{-}$

$==0 ะ 009000$.

ธ̄ธ-ธ. ๑00000000

$\stackrel{\circ}{\circ}$

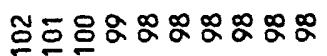

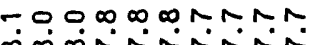
DoDNRMNRN

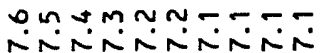
$\bar{\infty}$

mutor 00000 mimmiñ

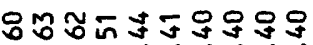
000000000<smiles>[Li]</smiles>

$\stackrel{\approx}{0}$

$m$

$\stackrel{\sim}{\sim}$

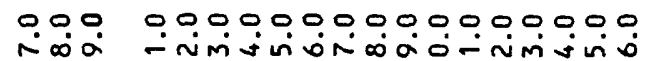

000000000

- Nimisivisió

0000000000 - invivivino

폴

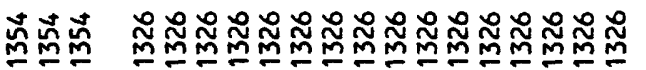

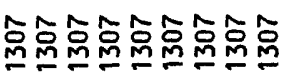

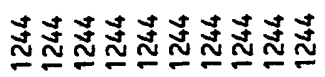

0000000000

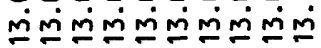




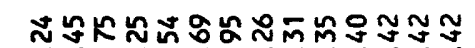

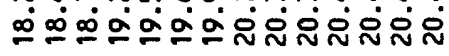

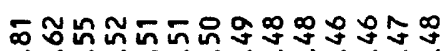

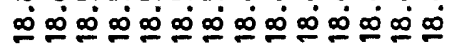

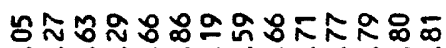

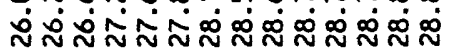

$\stackrel{\circ}{2}$

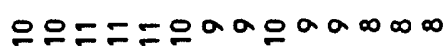

55555588888888 ó0000000000

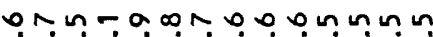
mimmiñiññ்iññ

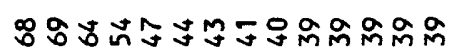
0000000000000

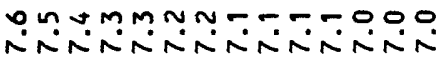

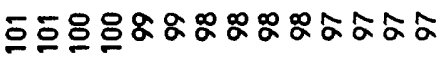

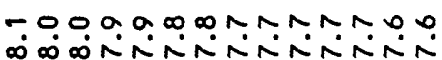

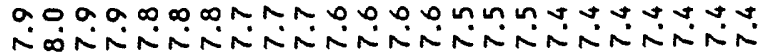

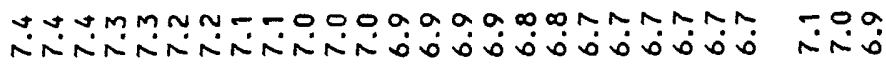

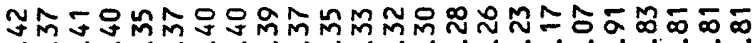

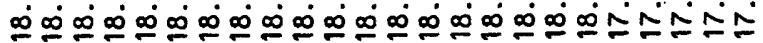

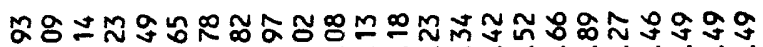

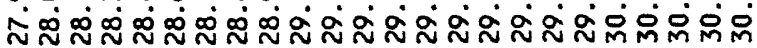
$\stackrel{\infty}{\circ}$

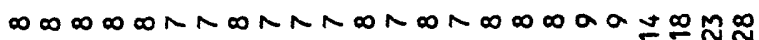
$\infty \infty N$

$888888888888888888885 \approx M$

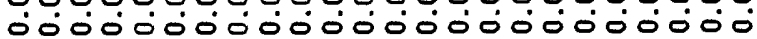

888 $\stackrel{\sim}{0}$

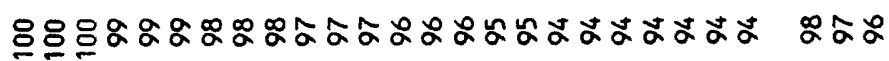

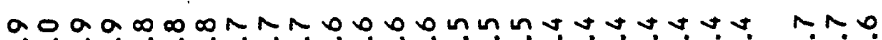

$\infty$

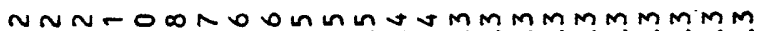

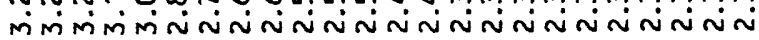

$a .0$.

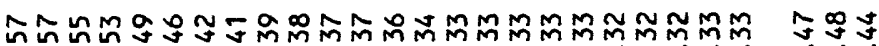
00000000000000000000 000

$\begin{array}{ll}\hat{N} & \stackrel{n}{0} \\ \dot{m} & \overline{0} \\ & \bar{i}\end{array}$

00000000000000

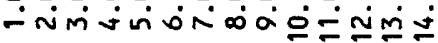

000000000000000000000000

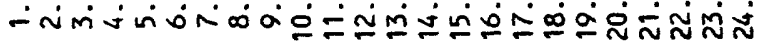

0.0

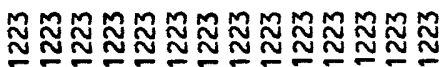

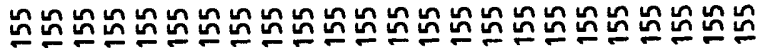

$\stackrel{n}{=} \cong$

00000000000000

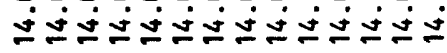

000000000000000000000000

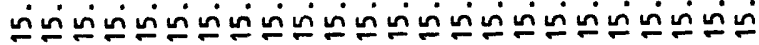


mำก8ำกำกับ ลักลสกสกำก

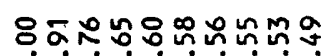
ФNㅗㅗㅗN

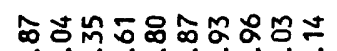

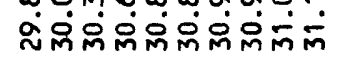

$\sim N \infty \sim \infty \infty 000$ 은

8888888888 000000000

8888888888888

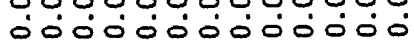

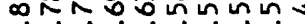

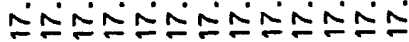

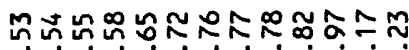

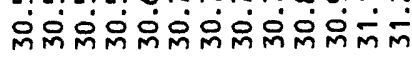
$\hat{o}$

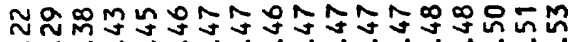

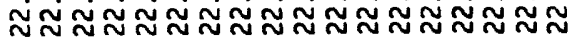

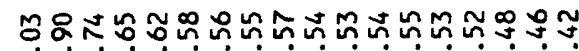

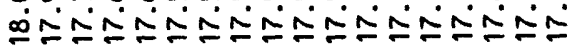

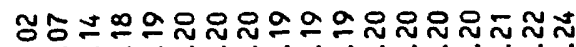

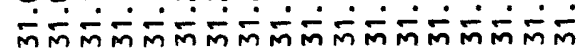

$\stackrel{a}{\circ}$

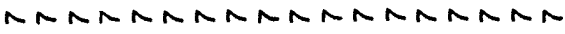

888888888888888888

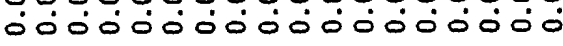

$\hat{n}$

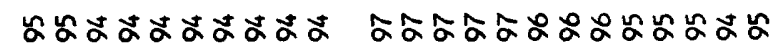

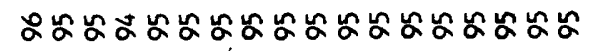

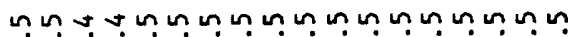

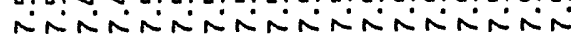

$\infty \infty \infty \infty \infty \infty \infty \infty \infty \infty, a \infty a, a, \infty$ ம $\dot{0}$ ல

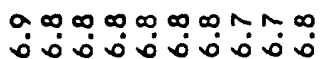

$-0.000,0000 \infty \infty$

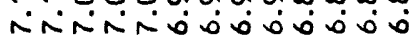

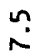

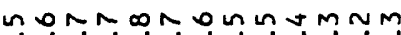

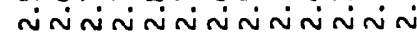



峞宅

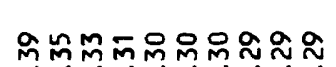

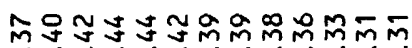
000000000 ०0ं000000000

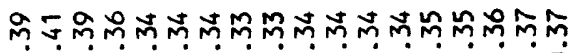
00000000000000000

in

0000000000000 - nimivivinas

000000000000000000 $\stackrel{0}{\circ}$ $\dot{m}$

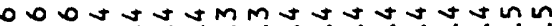

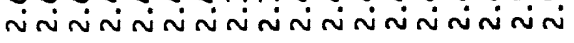

害

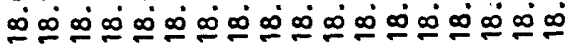




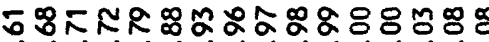

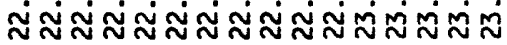

Mี

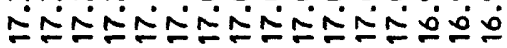

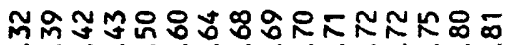

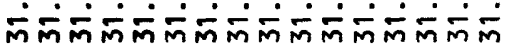

$\sim \wedge \sim \wedge \sim \infty \infty \infty \infty \infty \infty \infty \infty \infty \infty$

8888888888888888 ऽ000000000000

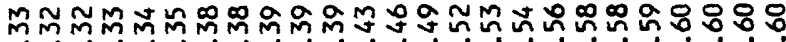

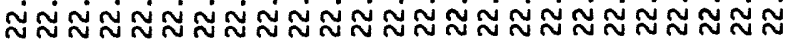

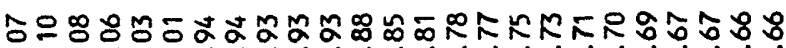

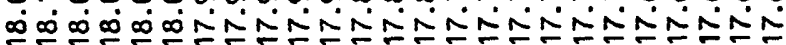

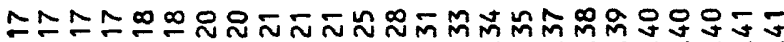

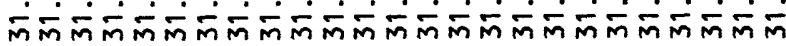
$\dot{0}$

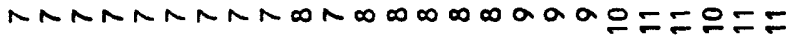

8888888888888888888855555

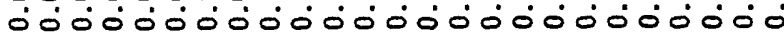

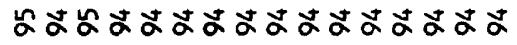

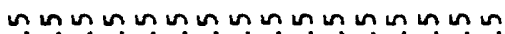

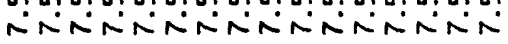

$\infty \infty \infty \infty \infty \infty \infty \infty \infty \infty \infty \infty \infty \infty \infty \infty$ $\dot{0} \dot{0} \dot{0} \dot{0} \dot{0} \dot{0} \dot{0} \dot{0} \dot{0} \dot{0} \dot{0} \dot{0} \dot{0} \dot{0}$

앵

옹롱

nงvg

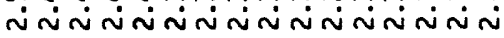

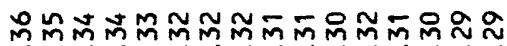
-00000000000000$$
\text { 空这: }
$$$$
\text { 芯룽 }
$$

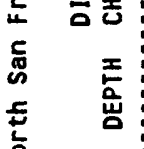

0000000000000000000000000

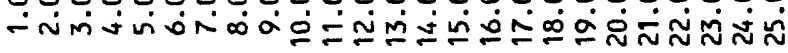

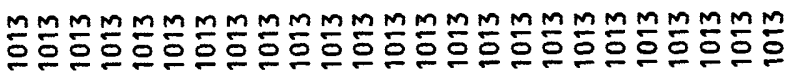

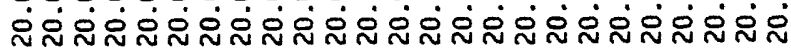

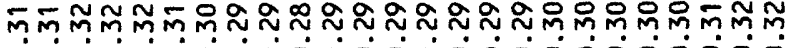

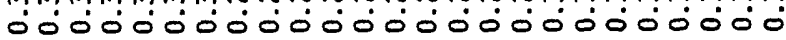

$M M M M m M \sim \sim N \sim N \sim \sim N \sim \sim \sim N \sim \sim N N M M M$

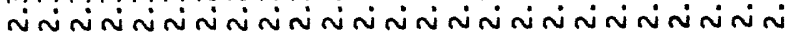

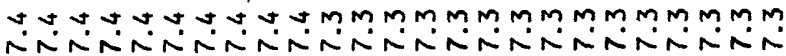
NNNNNNNNNR000000000000000

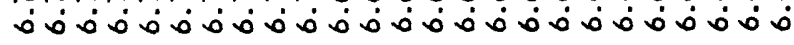




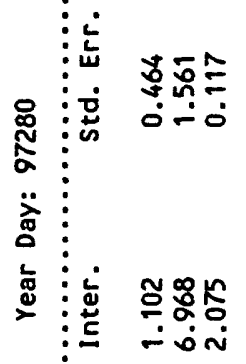

迹

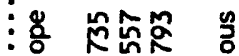

$\frac{0}{3}$

!

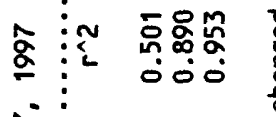

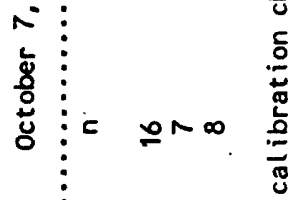

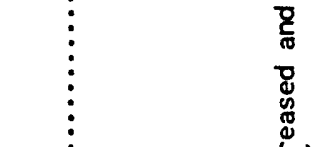

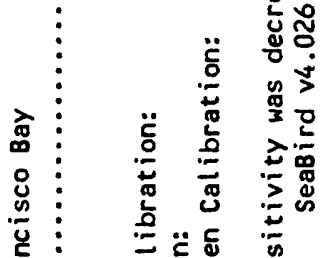

总

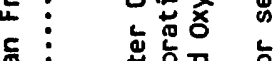

ङ

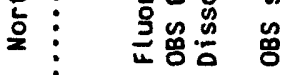




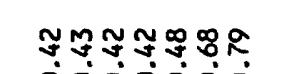

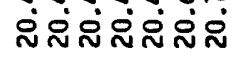

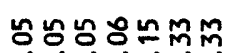

$\stackrel{\infty}{\infty} \propto \infty \omega$

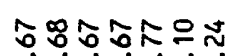

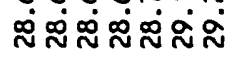

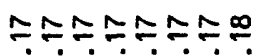

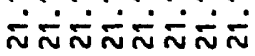

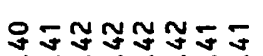

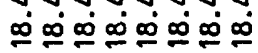

TRRNARR

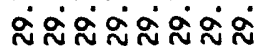

용ํㅇำ

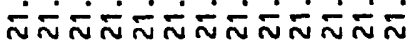

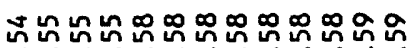

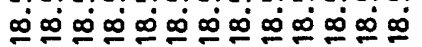

mํํำñ

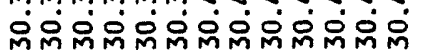

RRRRr?R?RR?R

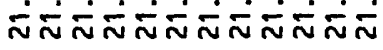

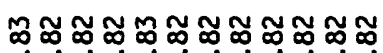

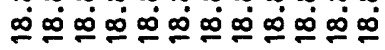

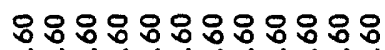

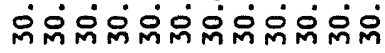

$\stackrel{\square}{ }$

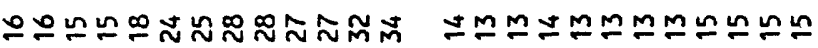

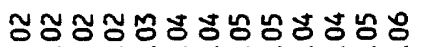
व.000.000.000.0

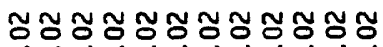
0ं000000000

$$
\stackrel{\circ}{=}
$$

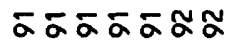

สะสะสะสั

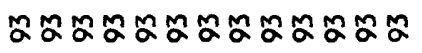

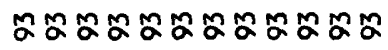

צח

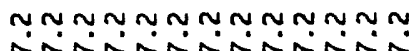

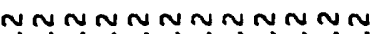
nanninaninar

aagaagaagaaaa

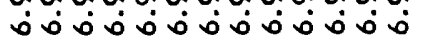

$\infty \infty \infty \infty \infty \infty \infty \infty a \alpha a$

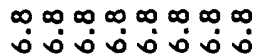

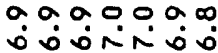

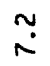$$
\stackrel{\sim}{\dddot{N}}
$$

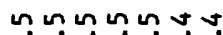
miniminimin

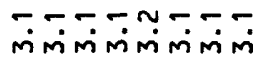

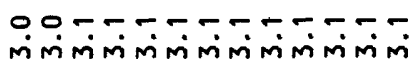

$\infty \infty \infty N-\infty \infty \infty \infty \infty \infty$

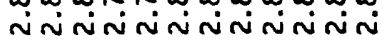

Кก⿻上丨刃

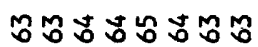
00000000

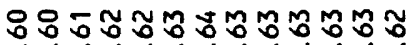
000000000000

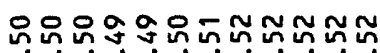
000000000000

$$
\text { ఫे }
$$

๘ั0

$\stackrel{\infty}{\sim}$

$\stackrel{\sim}{\sim}$

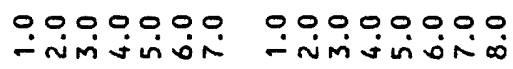

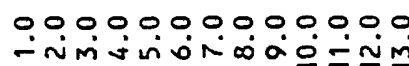

000000000000

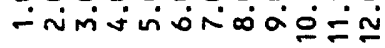

\section{$\frac{\infty}{8} \frac{\infty}{8} \frac{\infty}{8} \frac{\infty}{8} \frac{\infty}{8} \frac{\infty}{8}$}

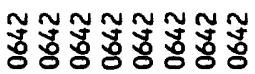

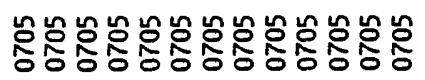

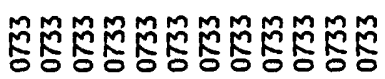

0000000000000

000000000000

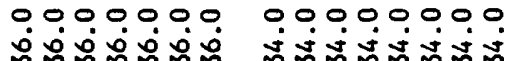

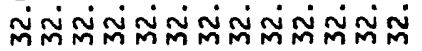

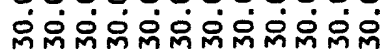




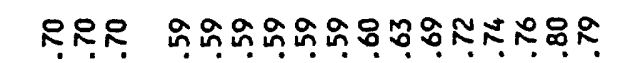

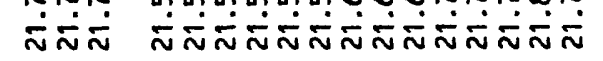

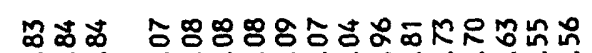

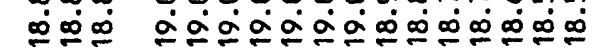

8.8 m

लिं户ि

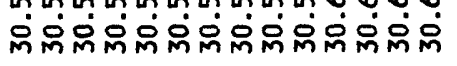
$\cong$

으음

$\widetilde{\sim}$ $\therefore 0^{\circ}$

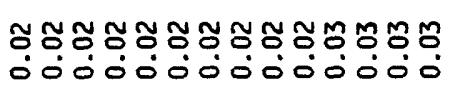

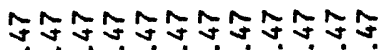

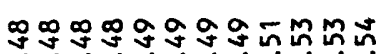

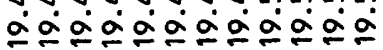

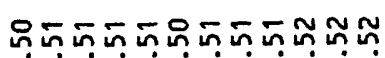

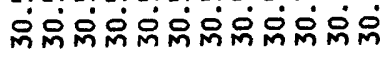

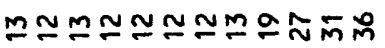

ชัญ

\section{$\infty \infty \infty$} inin

กุกนุกับ

$\circ 0^{\circ}$

:

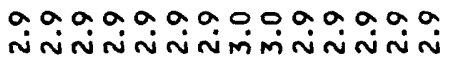

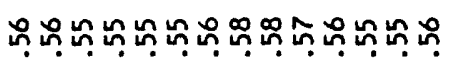
-0000000000000

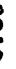

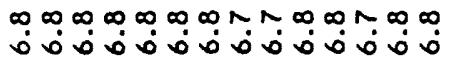

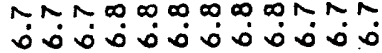

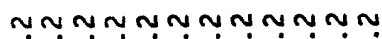

isinganding

$\dot{m} \dot{x}$

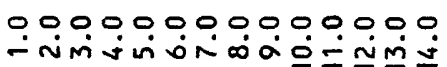

崖

\section{ํㅗㅇํㅗㅇ}

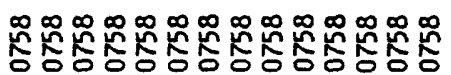

$0.000 .000,000,0000000$

z:

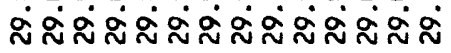

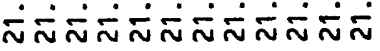

$\stackrel{\square}{\square}$

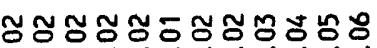
ด0ं00000000

๓ก

$\stackrel{\sim}{n}$

nunnannon

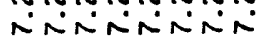

ñn

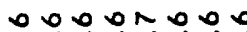

$\dot{0} \dot{0} \dot{0} \dot{0} \dot{0} \dot{0} \dot{0}$

$\infty \infty$ ?

NOOOONANRNMN

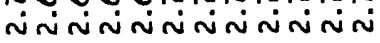

MMMmMMmM iñinaniñ

ñon

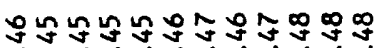

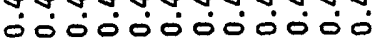

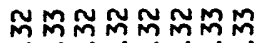
0.000000

m.

mọ

$\stackrel{̃}{0}$

$\stackrel{0}{0}$

$\hat{i}$

mi

$\stackrel{m}{\sim}$

000000000000

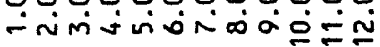

0,0000000

$\stackrel{\circ}{\circ}$

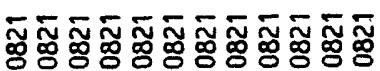

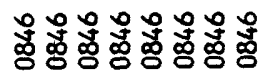

gू

0.00000000000

00000000

กก $\bar{N} \bar{N}$

$\stackrel{\infty}{\infty}$

$\underline{\alpha}$

๓๓ฒm

ำ่ำ

$\dddot{\square}$

$\infty \infty$

5 ?

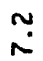

min O०

0.

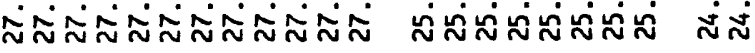


TKRำ

ஸ்ஸ்ர்

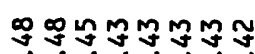

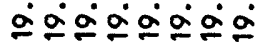

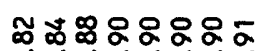

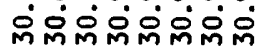

总

芯

爮产

\%ั

递窝

에 $\infty$ 으으으으

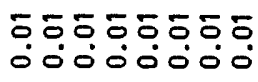

888888888888888888 ல0000000000000000

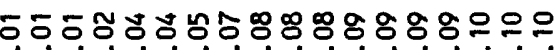

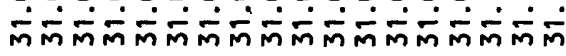

$\stackrel{\circ}{\circ}$

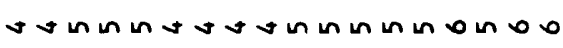

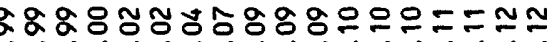

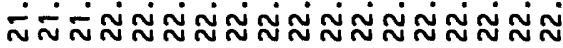

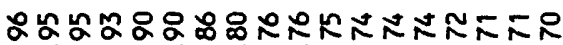

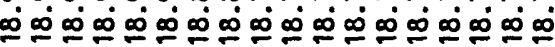$$
\text { (1) }
$$

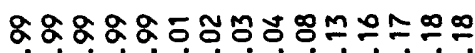

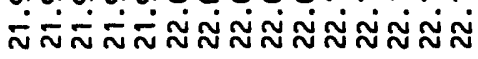

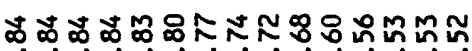

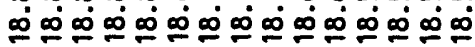

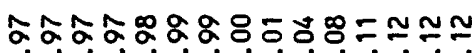

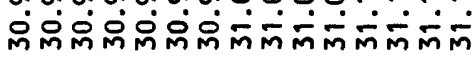

$\ddot{0}$

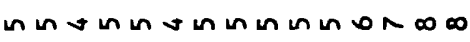

888888888888550

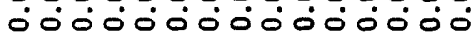

$\stackrel{\circ}{\circ}$

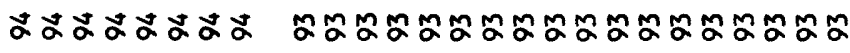

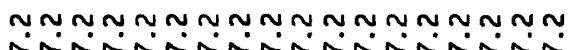

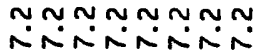

mannanha

ด.

0 0 0 0 0 0 n ○்

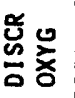

웛론

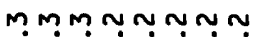

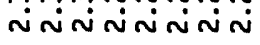

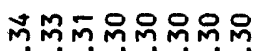
0000000

00000000

miviviniog

0.0000000000000000

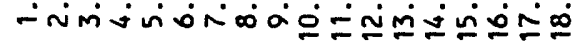

mmMmm บ N N N N NMmM

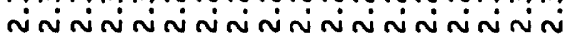

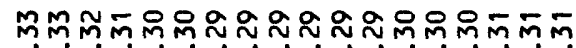
00000000000000000

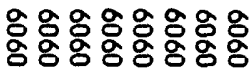

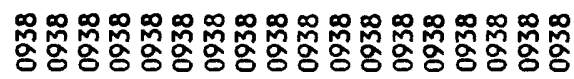

$0.000,0000$

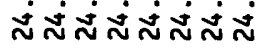

$00,000,000000000000$

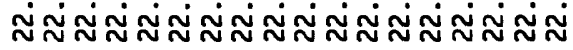

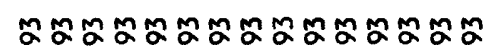

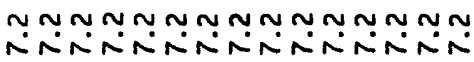

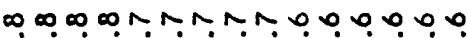

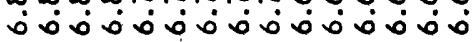

$\stackrel{\sim}{\dddot{N}}$

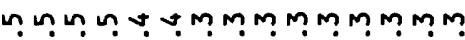

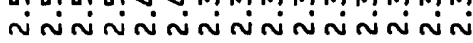

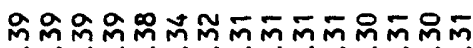
0000000000000

$\hat{0}$

m.

000000000000000 -

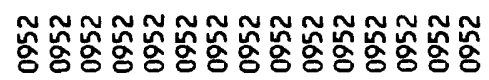

000000000000000

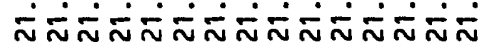




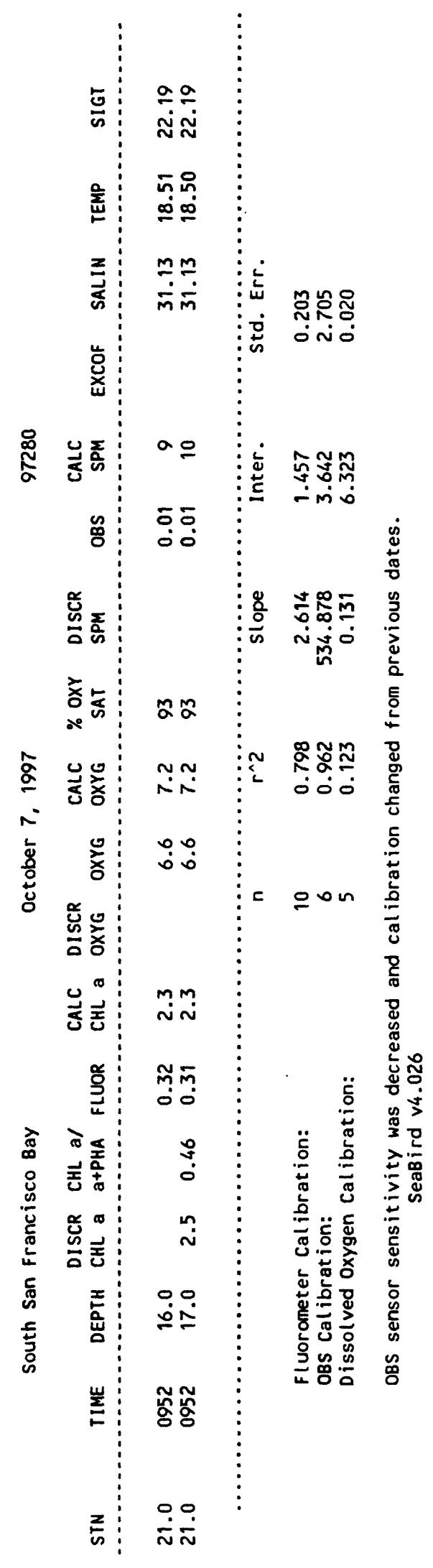


888888888888 ஸ000ं000000

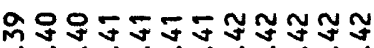

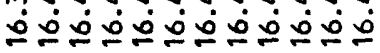

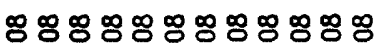
¿0000000000

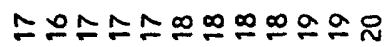

으으으으으으유=ㅠ

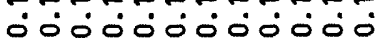

$$
\stackrel{\infty}{0}
$$

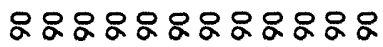

$\sim \sim \infty, \infty \infty \infty \infty \infty \infty \infty \infty$ $\dot{\infty} \dot{\infty} \infty \dot{\infty} \infty \dot{\infty} \infty \dot{\infty} \infty \dot{\infty} \infty \dot{\infty} \infty \dot{\infty}$

0.90.90000000

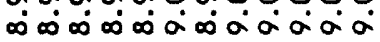

$$
\hat{\infty}
$$

$\sim \wedge \cap \infty \infty \infty \infty \infty \infty \infty \infty \infty$

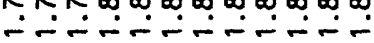

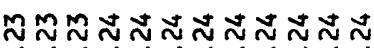
000000000000

$$
\begin{aligned}
& \text { on } \\
& 0 \\
& 0 \\
& \hdashline
\end{aligned}
$$$$
\stackrel{5}{0}
$$

000000000000

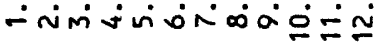

$\stackrel{\circ}{\sim}$

\section{$\frac{n}{8} \frac{n}{8} \frac{n}{8} \frac{n}{8} \frac{n}{8} \frac{n}{8} \frac{n}{8} \frac{n}{8} \frac{n}{8} \frac{n}{8} \frac{n}{8} \frac{n}{8}$

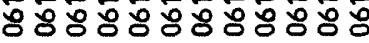

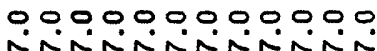

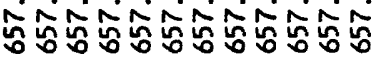

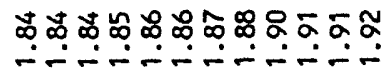

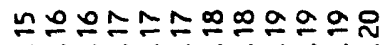

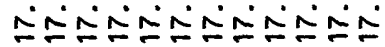

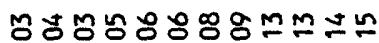

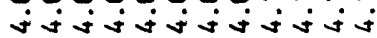

$\bar{i}$

$\stackrel{\circ}{-}$

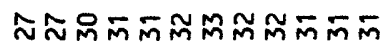

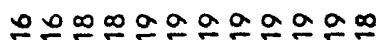
00000000000

ì

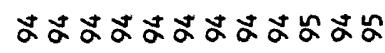

$\infty \infty \infty \infty \infty \infty \infty \infty \infty \infty a$ $\infty \infty_{\infty} \infty \dot{\infty} \infty \dot{\infty} \infty \dot{\infty} \infty \dot{\infty} \infty \dot{\infty} \infty \dot{\infty}$

000000000000

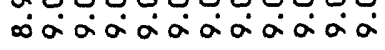

$\stackrel{a}{\infty}$

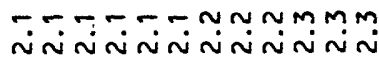

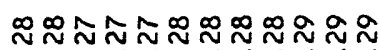
000000000000

?.

$\tilde{n}$

$\stackrel{\circ}{\sim}$

$\tilde{n}$

000000000000

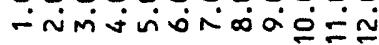

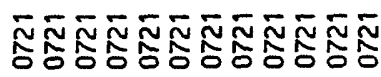

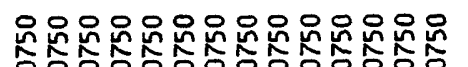

$00000-7 .-n n-7 n$ NiñNiñNiñNiñ

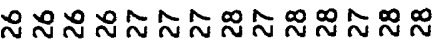

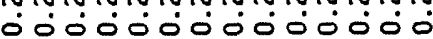

00000000000000

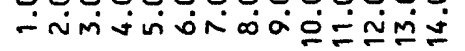

$\because 9$

กั๊ ๑๐

8 요

홍소 $\dddot{\sim}=$ $\stackrel{i}{i}$

พก

$\because 2$ $\therefore 0^{\circ}$

さす $\stackrel{\infty}{\infty} \underset{\infty}{\infty}$ no. $\infty \infty$$$
\text { . }
$$

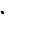

00000000000000 ininininininininin niv 


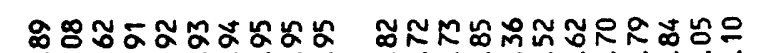
ก⿻

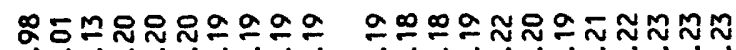

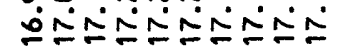

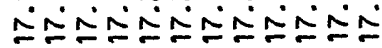

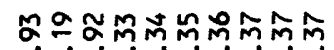

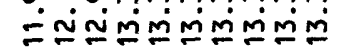

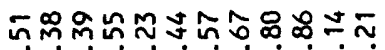
ปjป்

$\stackrel{\infty}{-}$

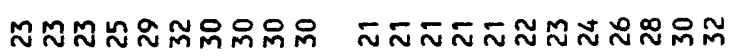

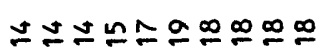

:

莒

爻产

\$

迅

$\circ$

蛋

총

음 옹

迅폰

告

i.

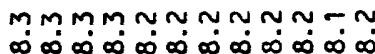

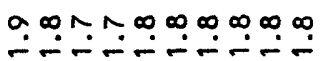

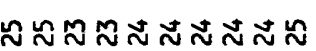

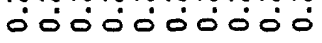

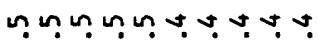
$\infty \infty_{\infty}^{\infty} \infty \dot{\infty} \infty \dot{\infty} \infty \dot{\infty} \infty \dot{\infty}$

$\stackrel{\sim}{\infty}$

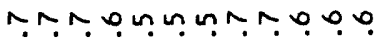

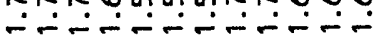

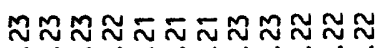
000000000000

ì

¿̊:

$\stackrel{\circ}{-}$

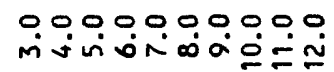

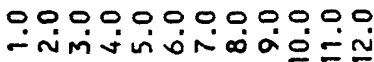

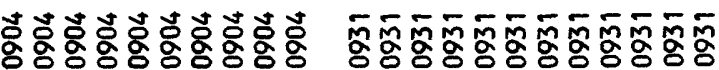

0000000000 minivivivivinivivinis
000000000000

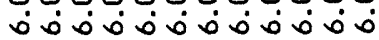

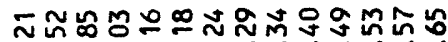

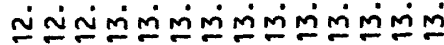

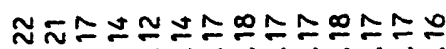

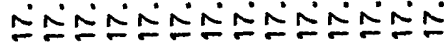

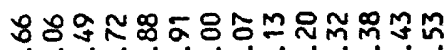

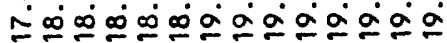

$\stackrel{+}{\square}$

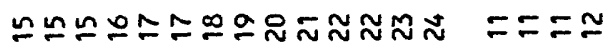

888800 000000000000

ะะะง ๑0ं0

よむるณ

$0009909090909 \%$

웅요

oooaagaagaaaaa क कo

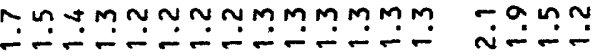

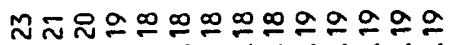

$\tilde{N} \cong$ ல000000000000

0.0ं

00000000000000

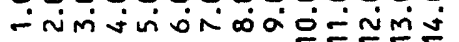

0.00

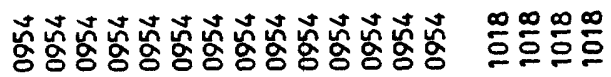

00000000000000

00000

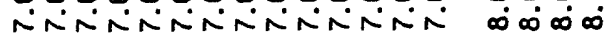




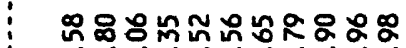

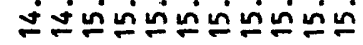

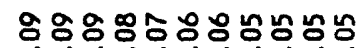

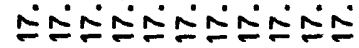

หํำํำำตำกำ

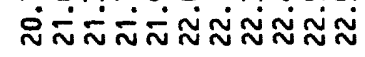

$\simeq \simeq \mathscr{M} M M M M \simeq M \pm$

ิㅗㅇํํㅇํㅇํㅇํํำ 000000000 

$\square \infty \infty \infty \infty \infty \infty \infty \sim N$

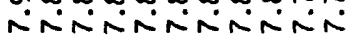

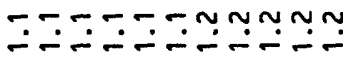

กニニ ニ ०0ं0000000

00000000000

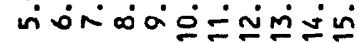

\section{$\simeq \infty \infty \infty \infty \infty \infty \infty \infty$}

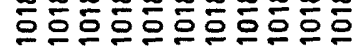

000.0000000 $\infty \dot{\infty} \infty \dot{\infty} \infty \dot{\infty} \infty \dot{\infty} \infty \dot{\infty} \infty \dot{\infty}$

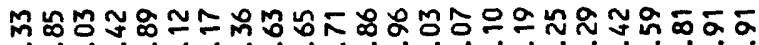

mต்

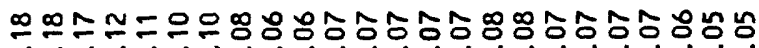

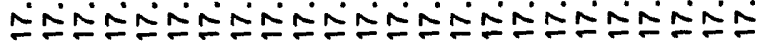

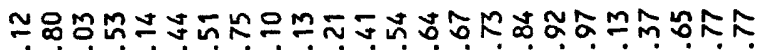

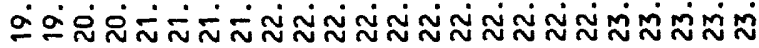

$\stackrel{m}{m}$

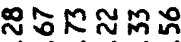

ษับํำำ

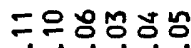

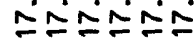

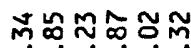

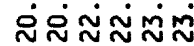

$\stackrel{m}{-}$

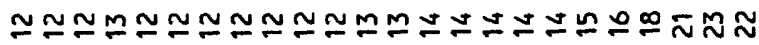

$=\simeq \cong \simeq \simeq 9$

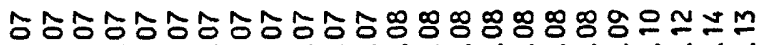

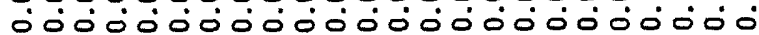

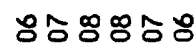
00000

$\cong$

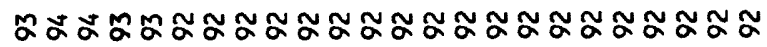

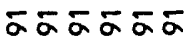

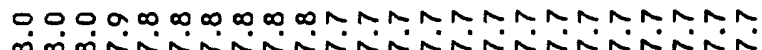

$\infty \infty \sim \sim 00$ intinin

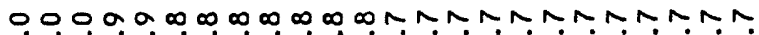

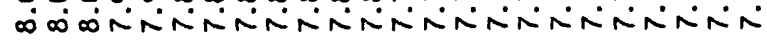

$\infty \infty N-\infty 0$ $\operatorname{cinting}$

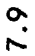

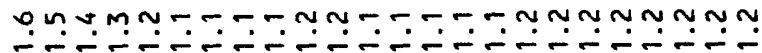

$m m ?-\frac{1}{m}$

กักํำ

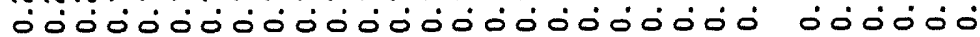

m

$\tilde{\imath}$

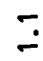

000000000000000000000000

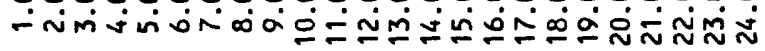

0.0900

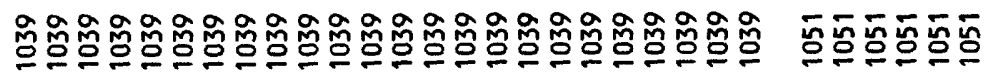

000000000000000000000000

0.0000 ㅇㅇㅇㅇㅇㅇ 


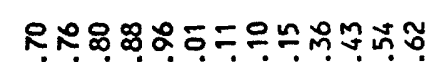

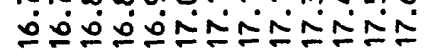

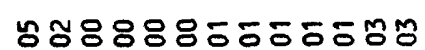

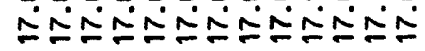

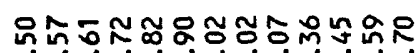

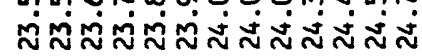

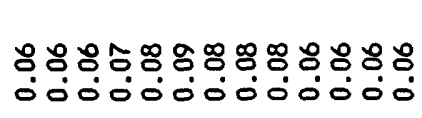

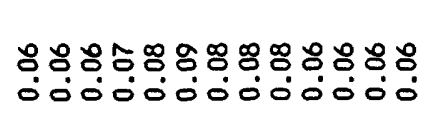

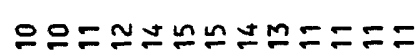

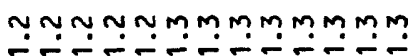

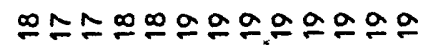
000000000000

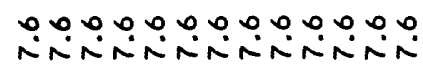

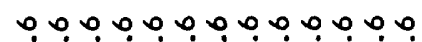

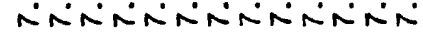

$\hat{N}$

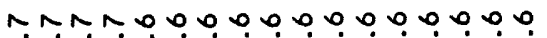

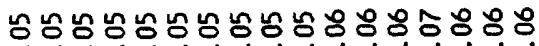
0000000000000000

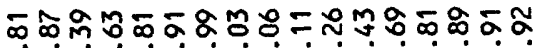

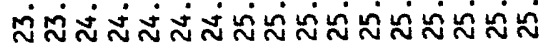

$\stackrel{-}{\circ}$

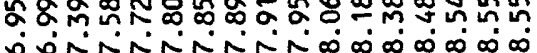

오소ำง

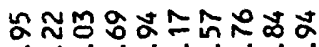

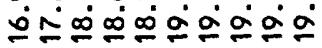

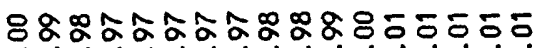

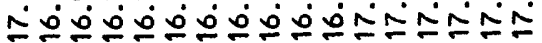

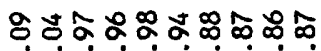
동ํㅇํㅇํㅇํㅇํㅇ

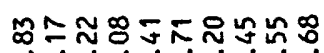

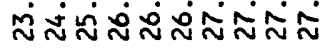

$\stackrel{9}{\circ}$

$\infty \infty \infty \infty \infty \wedge \sim \wedge \infty$

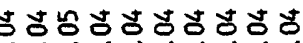
0.00000000

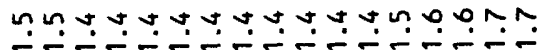

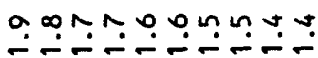

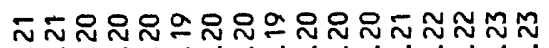
000000000000000

ผละก 0.000000000

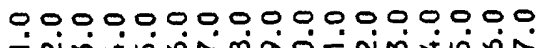

0000000000

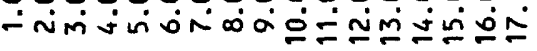

- nimivioñó 


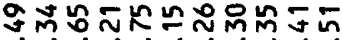
๓ ๓

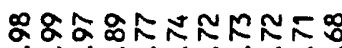

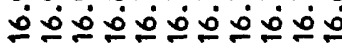

ณ ฝิ

$\stackrel{\infty}{\circ}$

$\frac{0}{n}$

खुํํำ

总

芦움

ชัธิ

$\hat{\alpha}$
0
0
8
0
0

ป̛

g.0.0.0.0.0.0nn n

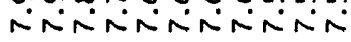

gampoopnnn

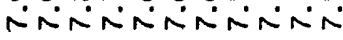

$\stackrel{\circ}{\infty}$

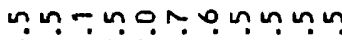
miminis:- - -

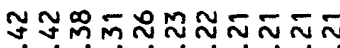
O0ं0ல00000

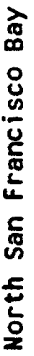

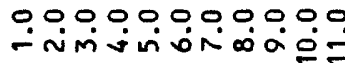

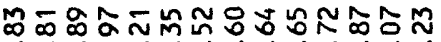

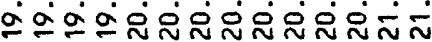

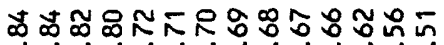

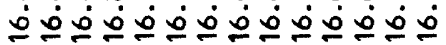

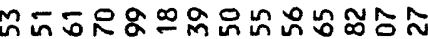
సิล்่ง்

$\stackrel{\infty}{0}$

○

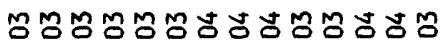
-00000000000

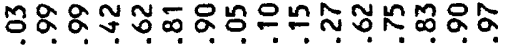

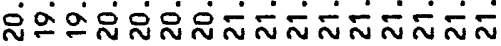

๒

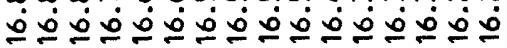

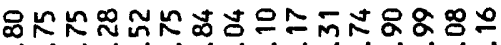

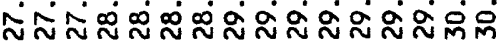

$\dddot{-}$

$\simeq \simeq \simeq=0,00 \infty \infty \infty \infty \wedge N R$

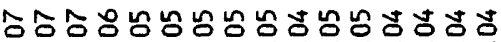

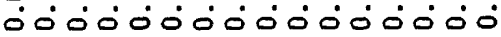

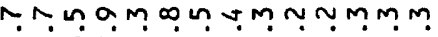

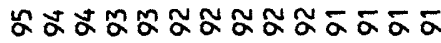

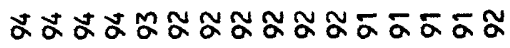

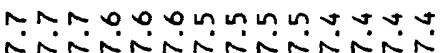

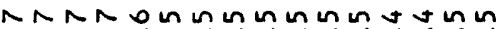

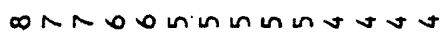

Niñom

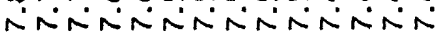

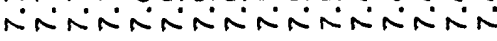

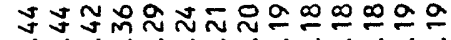
$\hat{i}$ 00000000000

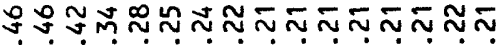
000000000000000
ڤ.
(1)
$\stackrel{m}{m}$

$0,0,0,000000,0000$

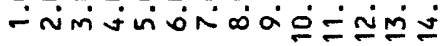

0000000000000000

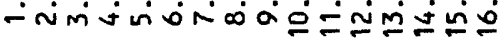

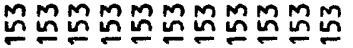

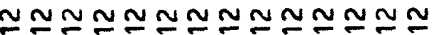

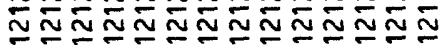

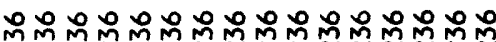

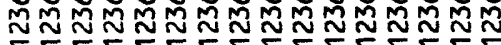




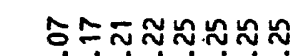
กำกำกำ

กำ우요

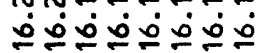

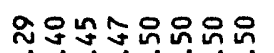
लिं

NoaNำ

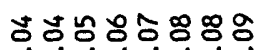
00000000

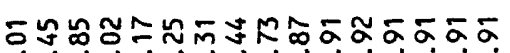
ฟล்

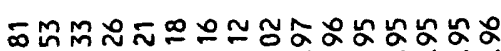

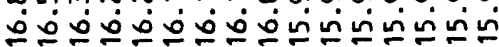

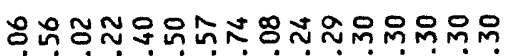

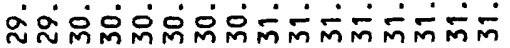

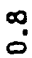

nแn m

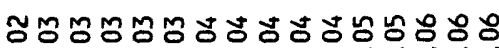
00000000000000

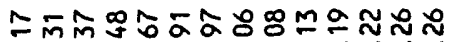

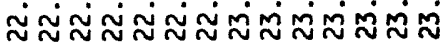

䒢

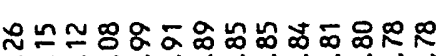

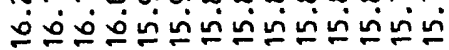

ㅇำ ทำ

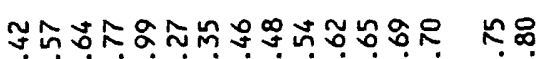

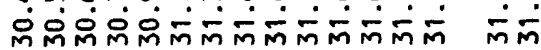
$\grave{o}$ $\hat{0}$

m num m

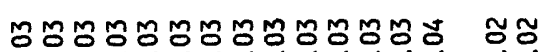
00000000000000 00

$\stackrel{\infty}{m}$

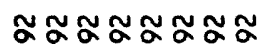

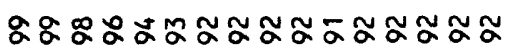

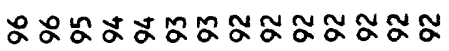

なa

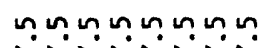

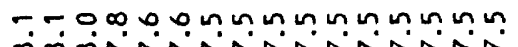

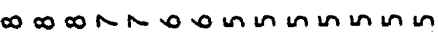

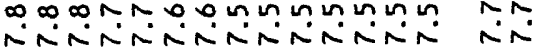

rinininis

क

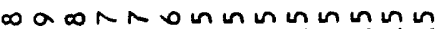

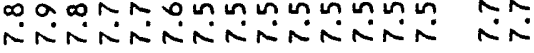

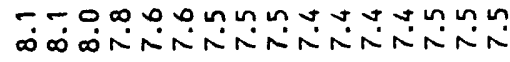

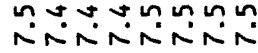

总

ํㅜㅇ

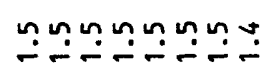

ำ

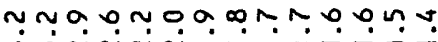

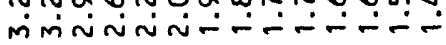

in

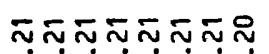

0000000

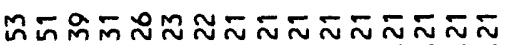
000000000000000

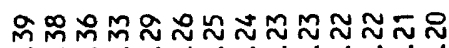

กัก

00000000000000

\section{0}

ڤ̊.

i

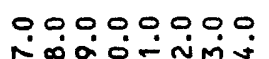

0.00000000000000

0,0000000000000

00

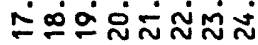

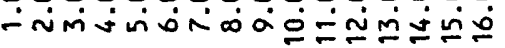

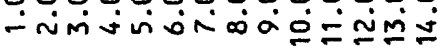

$-n$

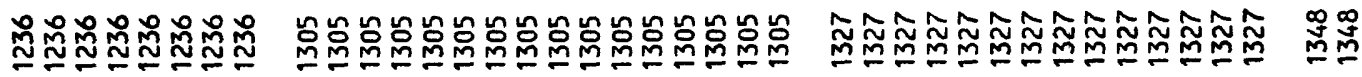

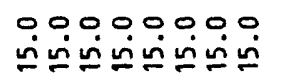

0000000000000000

00000000000000

0.0 


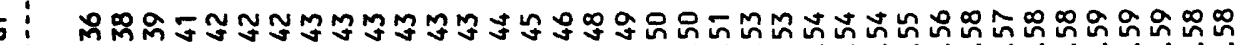

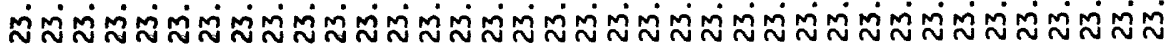

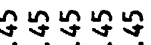

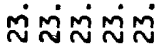

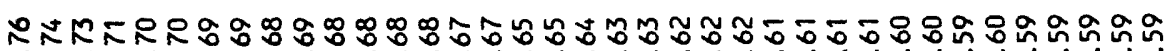

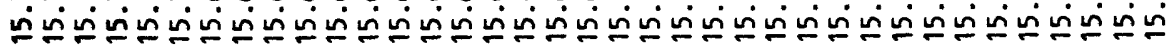

: ก่ำ่ำ

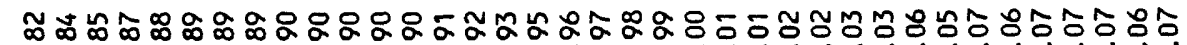

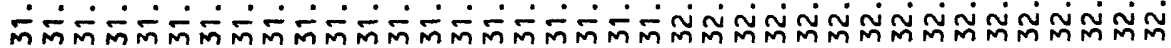

$\approx \approx \approx \approx \alpha$

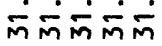

$\stackrel{\infty}{\infty}$

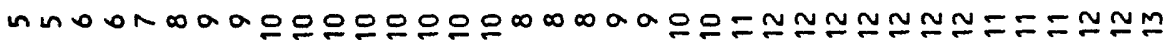

$\infty a \circ a a$

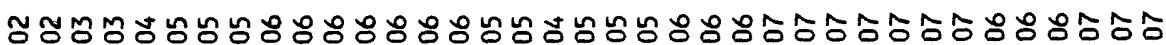

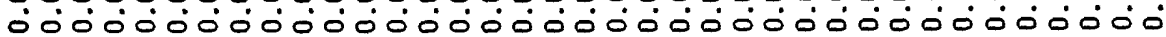

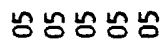
कंष்

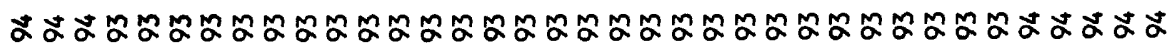

ฉะณณณ

NOO

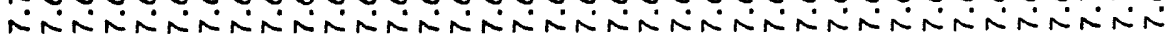

$\ln \ln \ln 0$

N000000000000000000000000000000000NAO riñín

$\ln \sin \sin \sin$

ไั่

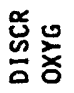

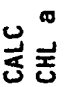

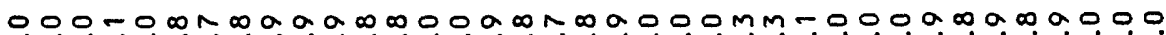

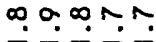
inini

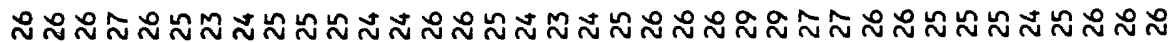

ฟ

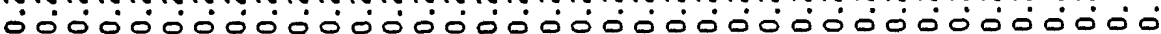

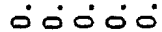

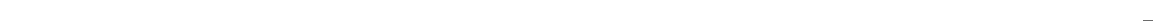




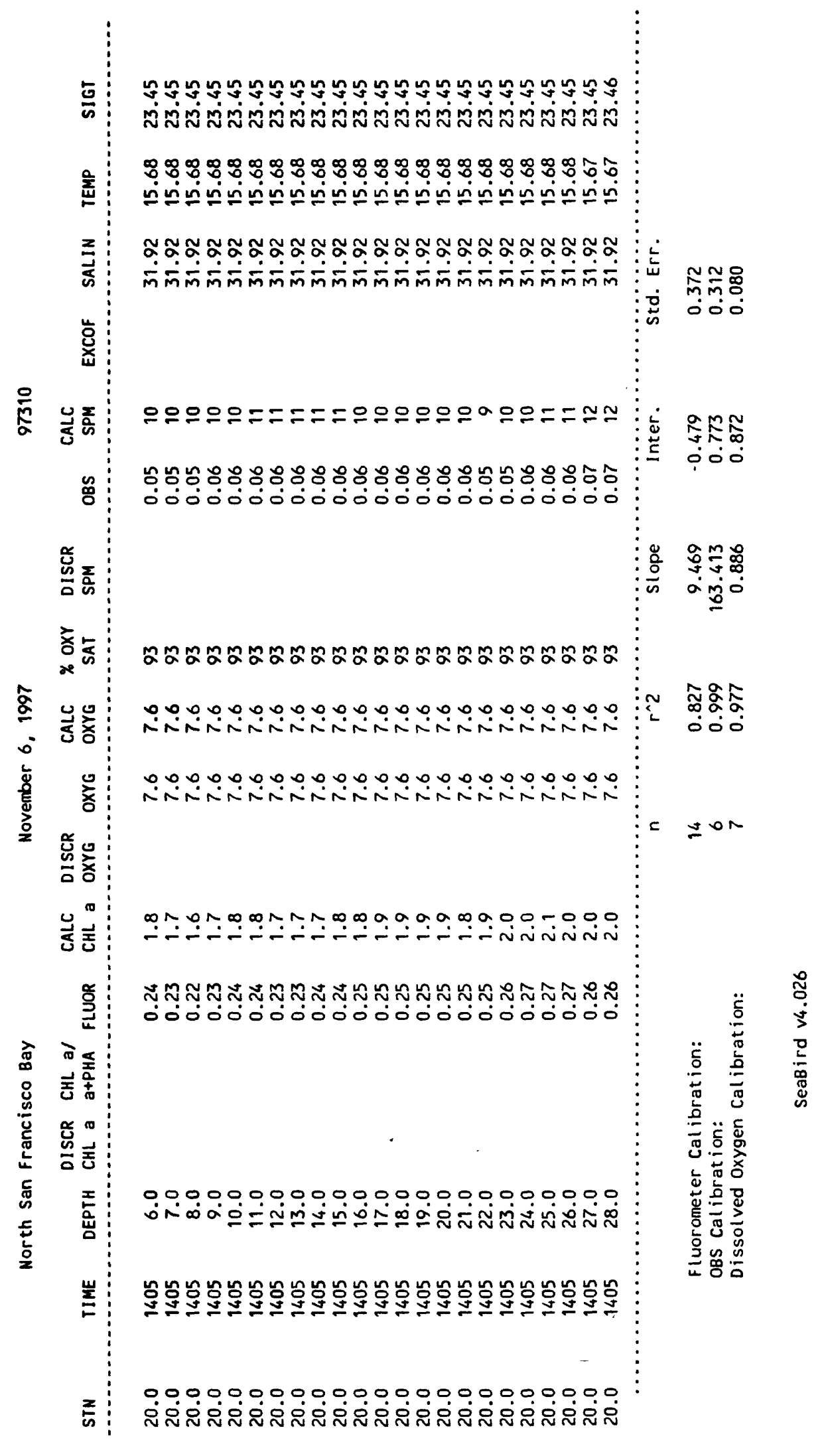




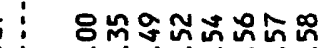

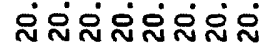

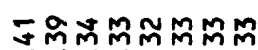

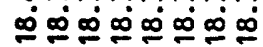

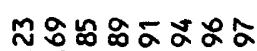

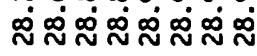

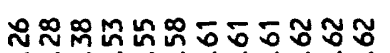

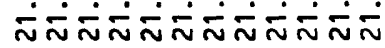

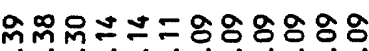

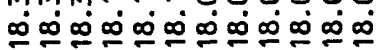

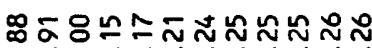

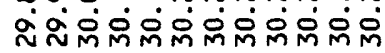

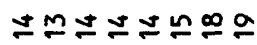

웅뭉영응 0ं00ं0000

$$
\dot{m}
$$

KKNNERRR

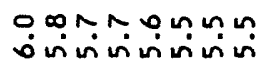

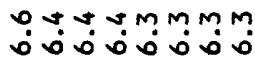
옳 응

岁究

mกดำกนก miñiññin

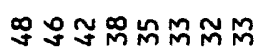
o0000000

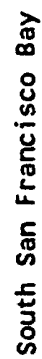

?

$\infty$

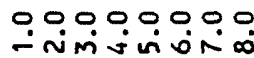

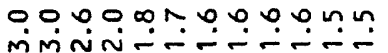

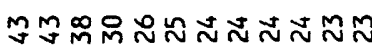
000000000000

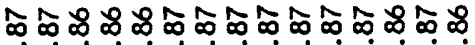

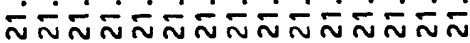

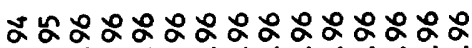

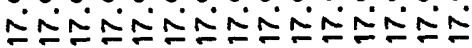

ผ

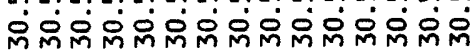

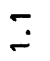

a a으으으으으으으 =ㅇㅇ으으으으

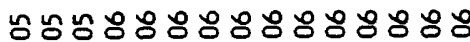

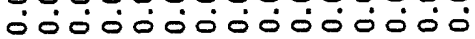

ชะะระ กี่กี่

ฆะลัลぇ ละกะล

용요요

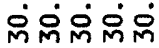<smiles>[CH]</smiles>

$\infty \infty \infty \infty \infty$

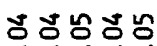
कं०ं०

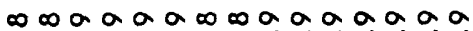

Un $⿻ m-$ minimi

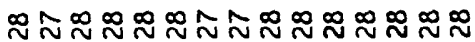

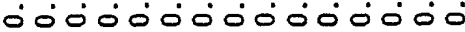

刓品学 वं००ण

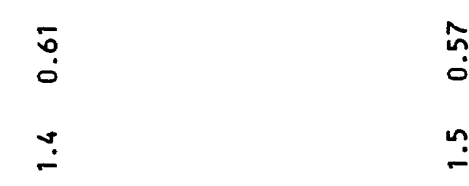

000000000000000

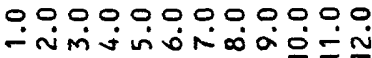

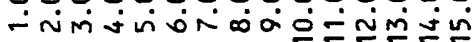

0.000 - invisin 
웅영영영ㅇㅇㅇㅇㅇㅇㅇ

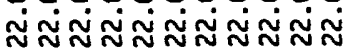

జு

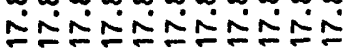

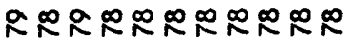

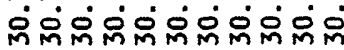

苞

a o ininininininin

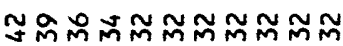

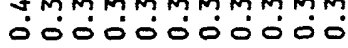

0.000 .000000 லri்

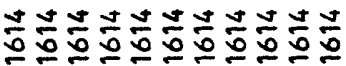

$g g=\simeq \simeq \simeq \simeq \simeq \simeq \simeq \square m$

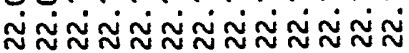

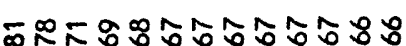

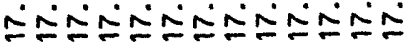

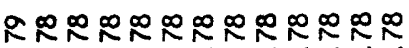

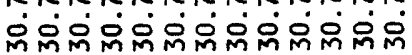

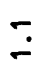

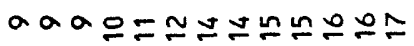

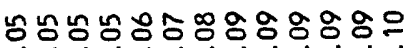
ó000000000

$\stackrel{\circ}{\circ}$

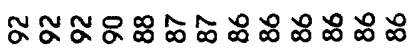

NMM-a $\infty \infty \infty \infty \infty \infty \infty$

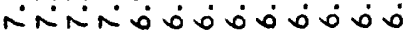

mmmn-

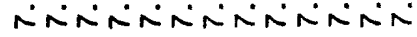

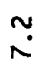

o $\infty$ MnN N NMmMmm ñiññniñññ

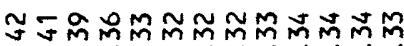
0ं0000000000

$\hat{\circ}$

జั.

$\stackrel{a}{i}$

$\hat{i}$

0000000000000

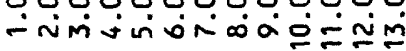

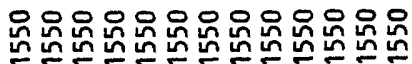

0.000000000000

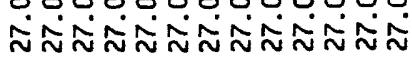

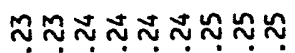

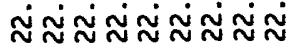

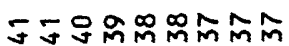
Nㅗㅗㅗㅗำ

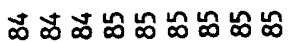

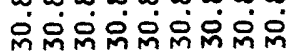

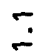

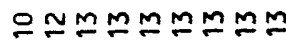

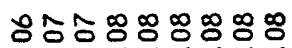
00000000

ㅇํำล์ิ์ 000000

$\stackrel{+}{=}$

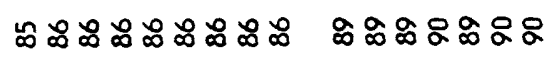
$\infty \infty \infty \infty \infty \infty \infty a$ فंல்.

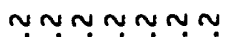

$0-0,007=5$

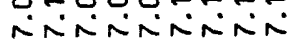

m?mmmm?

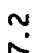

ง.ำmmmmm

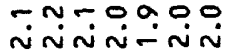

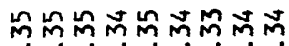
ơơ

ตำตำำำ 0000000

?

$\stackrel{\sim}{\sim}$

000000000 - imivinorióa

0.000000 - imivinion

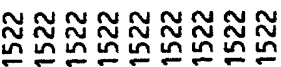

号贻总总总总总总

0.0000000

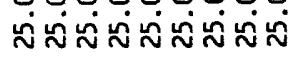

0000000

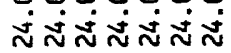




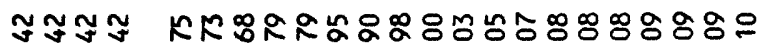

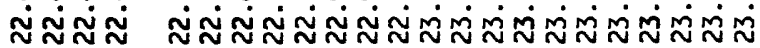

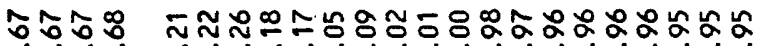
ㅇํำ

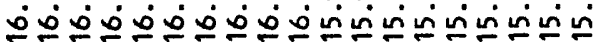

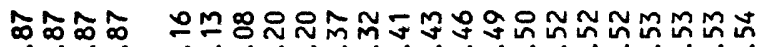

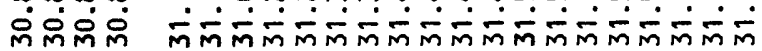
$\hat{0}$

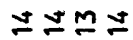

$\varnothing^{\circ} \varnothing^{\circ}$

$\dot{0} \dot{0} 0$

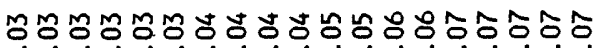
ó000000000000000

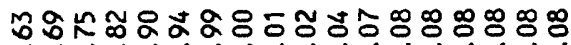

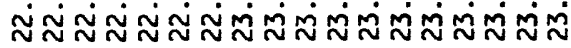

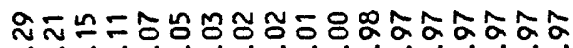

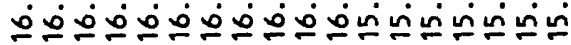

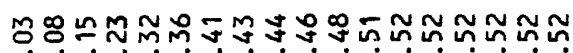

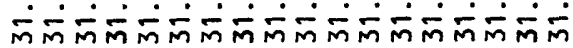

$\stackrel{\infty}{\circ}$

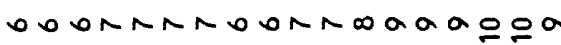

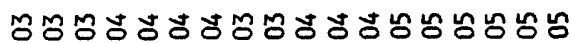
000000000000000

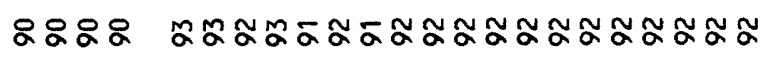

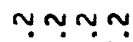

nNan

mimm

MNN

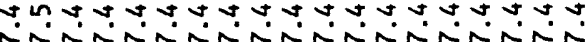

음

車

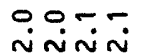

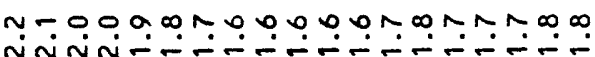

\section{ลิำำ}

000

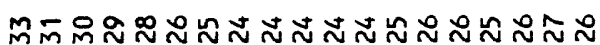
0000000000000000000 $\stackrel{\infty}{0}$

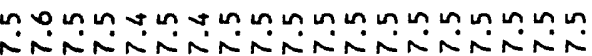

$\stackrel{\infty}{\sim}$

영요

0000000000000000000

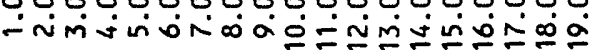

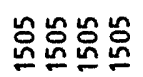

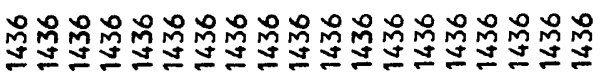

0.000 0000000000000000000

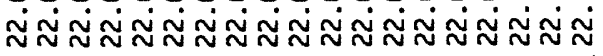

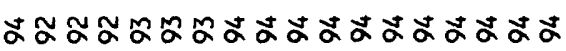

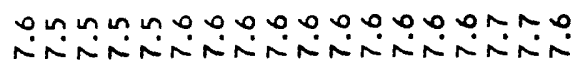

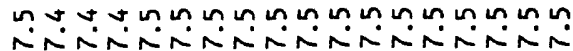
$\tilde{n}$

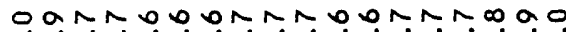

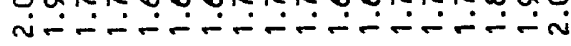

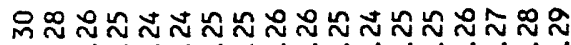
000000000000000000

$\bar{\infty}$

ก

000000000000000000 -

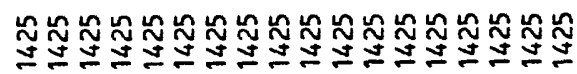

0,00000000000000000

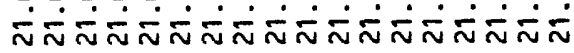




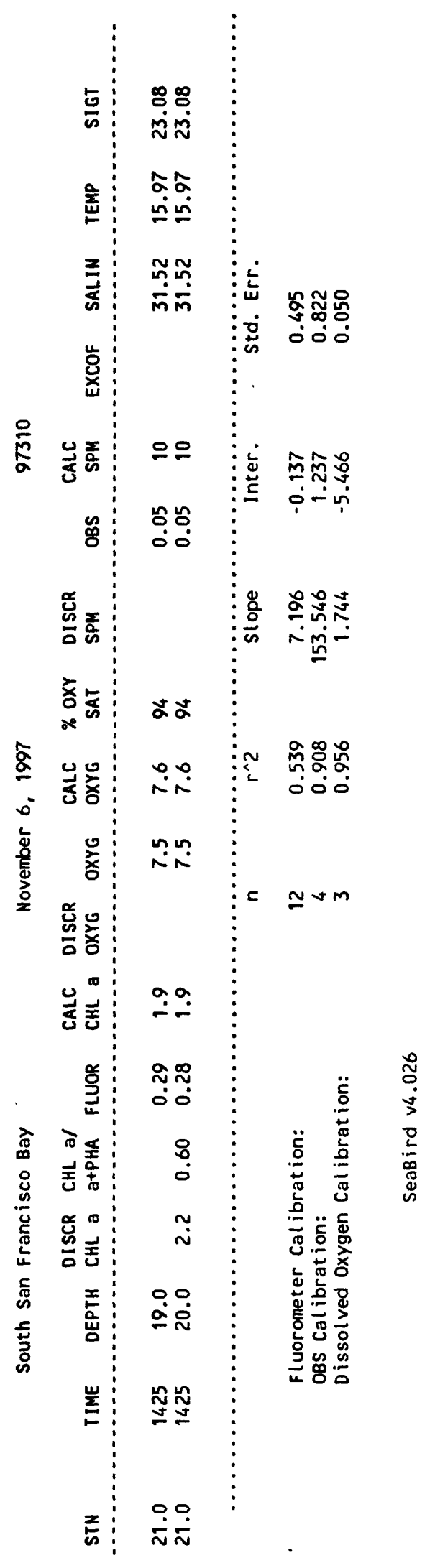

